FUNDAMENTA JURIDICA

Beiträge zur rechtswissenschaftlichen Grundlagenforschung

Leonie Breunung

\title{
Die Vollzugsorganisation als Entscheidungsfaktor des Verwaltungshandelns
}

Eine empirische Untersuchung zum

„vereinfachten Genehmigungsverfahren“" nach dem BImSchG

in Zusammenarbeit mit Hubert Treiber 
FUNDAMENTA JURIDICA

Beiträge zur rechtswissenschaftlichen

Grundlagenforschung

Band 38

Herausgegeben von

Jürgen Frank, Joachim Rückert (geschäftsführend),

Hans-Peter Schneider und Manfred Walther 
Leonie Breunung

in Zusammenarbeit mit Hubert Treiber

Die Vollzugsorganisation als

Entscheidungsfaktor

des Verwaltungshandelns

Eine empirische Untersuchung zum

„vereinfachten Genehmigungsverfahren"

nach dem BImSchG

Nomos Verlagsgesellschaft

Baden-Baden 
Erarbeitung und Drucklegung gefördert durch die Volkswagen-Stiftung.

Die Deutsche Bibliothek - CIP-Einheitsaufnahme

Ein Titeldatensatz für diese Publikation ist bei

Der Deutschen Bibliothek erhältlich. (http://www.ddb.de)

ISBN 3-7890-6978-7

1. Auflage 2000

(C) Nomos Verlagsgesellschaft, Baden-Baden 2000. Printed in Germany. Alle Rechte, auch die des Nachdrucks von Auszügen, der photomechanischen Wiedergabe und der Übersetzung, vorbehalten. Gedruckt auf alterungsbeständigem Papier. 


\section{Vorwort}

Die vorliegende, vergleichend angelegte empirische Untersuchung zum »vereinfachten Genehmigungsverfahren « nach dem Bundesimmissionsschutzgesetz (BImSchG) nutzt den Umstand, daß die Durchführung dieses Verfahrens in den einzelnen Ländern der Bundesrepublik unterschiedlich organisiert ist. Dabei ist auch unterschiedlich qualifiziertes Personal (Techniker bzw. Juristen/Verwaltungsfachleute) für die Genehmigungsentscheidung verantwortlich. Gegenübergestellt wurden eine Genehmigungsbehörde in Niedersachsen (dort identisch mit dem Staatlichen Gewerbeaufsichtsamt) und zwei entsprechende Behörden in Baden-Württemberg (im hiesigen Fall: zwei Landratsämter) mit dem jeweils dazugehörigen staatlichen Gewerbeaufsichtsamt als technischer Fachbehörde. Der Vergleich der Vollzugspraxis der beiden Genehmigungsbehörden auf der Basis einer Aktenanalyse sollte Aufschluß darüber erbringen, inwieweit sich die angesprochenen organisatorischen Rahmenbedingungen auf Dauer und Ergebnis der untersuchten Verfahren (Neu- wie Änderungsgenehmigungen) auswirken.

Es ist vornehmlich das Verdienst eines Amtsleiters im Norden, daß diese Untersuchung überhaupt zustande kam. Er gewährte uns nicht nur in seiner eigenen Behörde Akteneinsicht, sondern stellte auch die Kontakte zu den entsprechenden Behörden in Baden-Württemberg her. Die Zusicherung der Anonymität verbietet es, ihn namentlich hier zu nennen, wie wir aus diesem Grund auch darauf verzichten müssen, jenen Personen namentlich unseren Dank abzustatten, die in Süddeutschland das Projekt unterstützt haben. Ausdrücklich sei an dieser Stelle auch den mit den untersuchten Genehmigungsverfahren betrauten Sachbearbeitern gedankt, die beim Aktenziehen behilflich waren und geduldig die ihre Tätigkeit betreffenden Fragen beantwortet haben.

Nicht zuletzt ist ganz besonderer Dank der Volkswagen-Stiftung geschuldet, die Erarbeitung wie Drucklegung dieser Studie großzügig gefördert hat. Den Herausgebern der Fundamenta Juridica sei für die Aufnahme in die Reihe ebenfalls gedankt.

Hannover, im Januar 2000

Leonie Breunung/Hubert Treiber 
Die Fundamenta Juridica vereinigen seit 1985 Abhandlungen zur rechtswissenschaftlichen Grundlagenforschung. Wenn auch die Wissenschaftlichkeit der Jurisprudenz immer wieder umkämpft war und sein wird, wurde doch ebenso hartnäckig über den juridischen Tagesbetrieb hinaus nach Gründen und Zusammenhängen gefragt.

Für Analysen, die derart Gründe und Zusammenhänge aufgespürt haben, wollen die Herausgeber ein hilfreiches Forum schaffen, sie ermutigen und zusammenführen. Es gab und gibt viel Anlaß und Gelegenheit, im Grenzgang zwischen Jurisprudenz und den gesamten Sozialwissenschaften rechtswissenschaftliche Grundlagenforschung zu betreiben, alte Methoden neu zu erproben und neue kennenzulernen. Aus diesem immer noch jungen Prozeß sind eine Reihe grundlegender Arbeiten hervorgegangen.

Die Fundamenta Juridica sollen auch in Zukunft für solche Studien das äußere Band schaffen. Wegen des Tätigkeitsortes der Mehrheit der Herausgeber und wegen der beschriebenen Zielsetzung wird es sich vorwiegend um Hannoversche Beiträge handeln.

Hannover 1995

Die Herausgeber 
Inhaltsverzeichnis

$\begin{array}{ll}\text { I. Problemstellung } & 11\end{array}$

$\begin{array}{ll}\text { II. Anlage der Untersuchung } & 15\end{array}$

1. Erhebungsmethode 15

2. Auswahl der Genehmigungsbehörden 17

3. Auswahl der Verfahren 18

4. Randbedingungen der Verfahren 21

4.1. Behördliche Randbedingungen 21

4.2. Betriebliche Randbedingungen 25

4.2.1. Anlagenart 25

4.2.2. Unternehmensgröße 28

4.2.3. Externe Beratung 30

4.2.4. Genehmigungsart 32

$\begin{array}{ll}\text { III. Ergebnisse } & 37\end{array}$

1. Verfahrenseinleitung 37

1.1. Bekanntheit der behördlichen Zuständigkeit bei den Betreibern genehmigungspflichtiger Anlagen und der öffentlichen Verwaltung 37

1.2. Verfahrensauslösender Anlaß 40

1.3. Behördliche Vorantragsberatung 43

1.3.1. Häufigkeit von Vorantragsberatungen 43

1.3.2. Mitwirkung anderer Behörden 45

1.3.3. Medien der Kommunikation bei der
Vorantragsberatung

1.3.4. Gegenstände der Vorantragsberatung 52

2. Antragsbearbeitung (äußerlicher Verfahrensverlauf) 56

2.1. Vollständigkeitsprüfung der Antragsunterlagen 57

2.2. Unterrichtung des Antragstellers über den Gang des Verfahrens 58

2.3. Beteiligung von Fachbehörden und sonstigen Stellen 60 
2.3.1. Umfang der Fachbehördenbeteiligung 62

2.3.2. Art der beteiligten fachlichen Belange 66

2.4. Kommunikation zwischen Antragsteller und

Genehmigungsbehörde

2.4.1. Häufigkeit der Kontakte (Kommunikationsdichte)

2.4.2. Direkte und indirekte Beteiligung von Fachbehörden an der Kommunikation zwischen Antragsteller und Genehmigungsbehörde

2.4.3. Medium der Kommunikation zwischen Antragsteller und Genehmigungsbehörde

2.4.4. Gegenstände der Kommunikation

2.4.5. Behördliche Betriebsbegehungen bzw. -kontrollen

3. Entscheidungsverlauf

3.1. Sachliche Kontroversen zwischen den Hauptbeteiligten 95

3.1.1. Häufigkeit und Art der Kontroversen

3.1.2. Gegenstände und Ausgang immissionsschutzrechtlicher Kontroversen

3.1.3. Gegenstände und Ausgang nichtimmissionsschutzrechtlicher Kontroversen $\quad 106$

3.1.4. Einschaltung vorgesetzter Behörden 111

3.2. Revision von Genehmigungsentwürfen 113

3.3. Genehmigungsauflagen 121

3.3.1. Nicht-immissionsschutzrechtliche (sonstige) Auflagen

3.3.2. Immissionsschutzrechtliche Auflagen

3.4. Förmliche Widersprüche und ihr Ausgang

3.4.1. Häufigkeit von Widersprüchen

3.4.2. Ausgang der Widerspruchsverfahren (Entscheidung der Genehmigungsbehörde)

4. Verfahrensdauer(n)

4.1. Die Verfahrensdauer als Gegenstand von Kritik und Gesetzesreformen

4.2. Variationen der Gesamtdauer

4.2.1. Mögliche Bezugspunkte der Berechnung

4.2.2. Verschiedene Gesamtdauern im Vergleich

4.2.2.1. Dauer ab Vollständigkeit der Antragsunterlagen bis zum Erlaß des Genehmigungsbescheids

4.2.2.2. Dauer ab Antragseingang bis zum Erlaß des Genehmigungsbescheids 
4.2.2.3. Dauer ab erster Vorantragsberatung bis zum Erlaß des Genehmigungsbescheids 162

4.3. Dauern einzelner Verfahrensabschnitte 166

4.3.1. Vorantragsberatung (»Vorphase«) 167

4.3.2. Vollständigkeitsprüfung der Antragsunterlagen 170

4.3.3. Fachbehördenbeteiligung 173

4.3.3.1. Anforderung der Stellungnahmen durch die Genehmigungsbehörde (Fachbehördenbeiziehung) 174

4.3.3.2. Fachbehördliche Anfertigung der Stellungnahmen $\quad 180$

4.3.4. Erstellung des Genehmigungsbescheids $\quad 187$

4.3.4.1. Erarbeitung der Endfassung 187

4.3.4.2. Revision der Entwurfsfassung beim GAA-N 190

4.3.5. Widerspruchsbearbeitung (»Nachphase «) 192 


\section{Problemstellung}

Zur Implementation rechtlicher Programme durch die vollziehende Verwaltung liegt inzwischen eine umfängliche empirische Forschung aus unterschiedlichen rechtlichen Regelungsbereichen vor, darunter insbesondere auch zum Umweltschutzrecht. ${ }^{1}$ Den Anstoß dazu hatte das vielfach beobachtbare Auseinanderfallen von Norm und Realität, Programmzielen und deren tatsächliche Verwirklichung, gegeben. Die maßgebliche Ursache dafür wurde im Prozeß der Programmanwendung bzw. -durchführung vermutet. Die in den Untersuchungen allenthalben aufgedeckten, mehr oder weniger großen »Vollzugsdefizite « haben in der Folge zu einer Verlagerung des Forschungsinteresses geführt. Es geht weniger darum, Abweichungen der Verwaltungspraxis von der »buchstabengetreuen « Umsetzung der rechtlichen Vorschriften als solchen nachzugehen, ${ }^{2}$ als vielmehr darum, in welcher Art und Weise die Verwaltung in Ausübung ihrer Vollzugsaufgaben die in jedem Rechtsprogramm nahezu unvermeidlich enthaltenen Handlungs- und Entscheidungsspielräume nutzt oder auch über das anscheinend eindeutig rechtlich Zulässige hinaus erweitert und welche rechtlichen Ergebnisse und tatsächlichen Verhältnisse sie damit im jeweiligen Regelungsbereich herstellt. ${ }^{3}$ Diese Fragestellung trifft sich mit einem veränderten Bild der heutigen, modernen Verwaltung. Die klassische Zuschreibung der passiven Rolle als eines bloßen staatlichen Vollzugsorgans wird abgelöst durch die Akzeptanz - wenn nicht gar Einforderung - einer aktiven, gestaltenden Funktion, wie sie in den inzwischen geläufigen Bezeichnungen der »flexiblen «, »kooperativen « oder auch »verhandelnden $\ll^{4}$ Verwaltung zum Ausdruck kommt.

1 Als Beispiele für letztere seien die Untersuchungen von Winter 1975, Mayntz et al. 1978, Bohne 1981 genannt.

$2 \mathrm{Da}$ solche »Abweichungen« nicht erst ein Merkmal heutiger Verwaltungspraxis sind, sondern sich auch für die des 19. Jahrhunderts nachweisen lassen, zeigt Treiber 1989 am Beispiel des gewerblichen Arbeitsschutzes. Einen Überblick über die Hauptursachen von Vollzugsproblemen im heutigen Umweltrecht geben Huckel Wollmann 1994. Vgl. auch Lübbe-Wolff 1993 und Lorenz 1991.

3 Damit wird auch einem theoretischen Grundproblem der Vollzugsforschung Rechnung getragen. Denn wie der »buchstabengetreue« Gesetzesvollzug als Vergleichsmaßstab im jeweiligen konkreten Anwendungsfall auszusehen hätte, ist kein (wissenschaftlich lösbares) Erkenntnisproblem, sondern ein juristisches Entscheidungsproblem, für dessen verbindliche Lösung letztlich die Gerichte zuständig sind. Zu den theoretischen Grundlagen des Implementationsansatzes vgl. Mayntz 1980.

4 Unter diesen Titel stellt Dose 1997 seine Untersuchung zum Vollzug des Immissionsschutzrechts. 
Die vorliegende Untersuchung wendet sich dem Implementationsprozeß in erster Linie unter dem strukturellen Gesichtspunkt zu, wie sich die Organisation des verwaltungsförmigen Gesetzesvollzugs auf Verlauf und Ergebnis des Entscheidungsprozesses auswirkt. Das Interesse richtet sich dabei weniger auf Fragen der »Gesetzeskonformität« und möglicher »Vollzugsdefizite « der unter unterschiedlichen organisatorischen und personellen Rahmenbedingungen agierenden Vollzugspraxis als vielmehr auf die im »Schatten« der gesetzlichen Vorgaben jeweils entwickelte Ausgestaltung des Verwaltungshandelns sowie der für den Vollzug jeweils in Anspruch genommenen Zeit bzw. der jeweiligen Dauer der Verfahren.

Als geeigneter Gegenstand, dieser Frage empirisch nachzugehen, bot sich das »vereinfachte Verfahren « an, das das Bundesimmissionsschutzgesetz ( $\$ 19$ BImSchG) für die Genehmigung zur Errichtung und zum Betrieb bestimmter Anlagen zum Schutz der Umwelt vorsieht. Diese Verfahren bieten gegenüber den Regelverfahren, in denen die Öffentlichkeit zu beteiligen ist, ${ }^{5}$ nicht nur den Vorzug, den Routinebetrieb des Verwaltungsalltags erfassen zu können. Darüber hinaus und ausschlaggebend für ihre Wahl war der Umstand, daß die für den Verwaltungsvollzug des BImSchG zuständigen Bundesländer für die vereinfachten Verfahren unterschiedliche Modelle entwickelt haben. ${ }^{6}$ Ein wesentlicher Unterschied zwischen den Vollzugsmodellen, auf den sich die vorliegende Untersuchung konzentriert, betrifft die für die Genehmigungserteilung jeweils zuständige Behörde. In einigen Ländern wie Baden-Württemberg liegt die Zuständigkeit und damit die Verfahrenshoheit bei den unteren Behörden der allgemeinen Verwaltung, d.h. bei der »Auftragsverwaltung « der Landkreise und kreisfreien Städte. In anderen Ländern wie Niedersachsen liegen Genehmigungszuständigkeit und Verfahrenshoheit bei den für den Immissionsschutz zentralen technischen Fachbehörden, den staatlichen Gewerbeaufsichtsämtern, denen diese Funktion zusätzlich zu ihren fachtechnischen Aufgaben übertragen ist. ${ }^{7}$ In beiden Modellen hat allerdings die zuständige Genehmigungsbehörde nach dem BImSchG in jedem Fall die Pflicht, in Vorbereitung ihrer Entscheidung von anderen Behörden, deren Belange durch das anstehende Vorhaben betroffen sind, fachliche Stellungnahmen einzuholen. Somit ist die für den Immissionsschutz zentrale technische Fachbehörde, das staatliche Gewerbeaufsichtsamt, immer in das Genehmigungsverfahren eingebunden. Über die Fachbehördenbeteiligung erlangen die Bedingun-

5 Zum Unterschied zwischen vereinfachten und Regelverfahren (»förmliche Verfahren«) vgl. näher Harder 1987, S. 10ff.

6 Eine entsprechende Übersicht über die zum Zeitpunkt der vorliegenden Untersuchung praktizierten Modelle enthält Pütz/Buchholz 1991. Zu den wichtigsten Unterschieden der Modelle vgl. ausführlicher Lübbe-Wolff 1996, S. 55ff. und Dose 1997 , S. $231 \mathrm{ff}$.

7 In beiden Ländern gibt es jeweils nur wenige Ausnahmen, die bestimmte Anlagearten mit anders geregelter behördlicher Zuständigkeit betreffen. 
gen und Auflagen, die die Einhaltung der gesetzlichen Vorgaben sicherstellen sollen, ein für die Genehmigung entscheidendes Gewicht.

Die unterschiedliche behördliche Genehmigungszuständigkeit geht mit unterschiedlicher formaler fachlicher Qualifikation bzw. Kompetenz des entscheidungsbefugten Personals einher. Dieses besteht bei den für den Immissionsschutz verantwortlichen Ämtern der unteren allgemeinen Verwaltung aus ausgebildeten Verwaltungsfachleuten (in Baden-Württemberg mit einem 6-semestrigen Studium an einer landeseigenen Fachhochschule) ohne formale technisch-naturwissenschaftliche Qualifikation, bei den staatlichen Gewerbeaufsichtsämtern aus Technikern, Ingenieuren oder Naturwissenschaftlern mit zusätzlicher (im Rahmen eines Referendariats absolvierter) Verwaltungsausbildung. Die unterschiedliche berufliche Herkunft der Entscheidungsträger in den beiden Modellen läßt Auswirkungen auf die jeweilige Genehmigungspraxis insofern vermuten, als den technisch ausgebildeten Personen der Gewerbeaufsicht »schon von ihrer beruflichen Qualifikation her die ingenieurmäßige Beratung vor Ort« eher zusagen dürfte als die mehr in die genuine Kompetenz der Verwaltungsfachleute fallenden »bürokratischen Formen des umweltrechtlichen Vollzugs. ${ }^{8}$

Die vorliegende Untersuchung unternimmt eine vergleichende Analyse der genehmigungsrechtlichen Vollzugspraxis am Beispiel zweier badenwürttembergischer Landratsämter einerseits ${ }^{9}$, die für das Modell der Genehmigungszuständigkeit bei den Landkreisen und kreisfreien Städten mit Verwaltungsfachleuten als Entscheidungsträgern stehen, und am Beispiel eines niedersächsischen staatlichen Gewerbeaufsichtsamts andererseits, das das Modell der organisatorischen Integration von Genehmigungszuständigkeit und für den Umweltschutz maßgeblicher technischer Fachbehörde mit naturwissenschaftlich-technisch qualifizierten Entscheidungsträgern verkörpert.

Im Hinblick auf diesen Vergleich erscheint es angebracht, den Begriff »Gesetzesvollzug « zu differenzieren und zwei Vollzugsstufen zu unter-

8 Vgl. Lübbe-Wolff 1992, S. 231. Ähnliche Vermutungen finden sich bei Dose 1994, S. 231. Die unterschiedliche professionelle Orientierung von Technikern und Beamten der allgemeinen Verwaltung heben auch Mayntz et al. 1978, S. 252f. hervor. Allgemein zu den fachspezifischen Unterschieden in Denk- und Arbeitsweisen (»Habitus«) zwischen Ingenieuren und Juristen vgl. die Untersuchung von Mai 1989, die auf einer empirisch gestützten Analyse wissenschaftlicher Politikberatung der beiden Disziplinen im Bereich des Umweltschutzes beruht.

9 Genaugenommen handelt es sich jeweils um die für immissionsschutzrechtliche Genehmigungen zuständige, im Amt für Umweltschutz der Kreisverwaltung ressortierende untere Immissionsschutzbehörde. Da es in der Untersuchung um die unterschiedliche Zuordnung der Genehmigungskompetenz zur (unteren) allgemeinen Verwaltung einerseits und zur staatlichen Sonderverwaltung andererseits geht, wird nachfolgend die baden-württembergische Genehmigungsbehörde vereinfacht als Landratsamt bzw. abgekürzt als LRA bezeichnet. 
scheiden. ${ }^{10}$ Eine erste Stufe betrifft die behördliche Genehmigungsentscheidung als Anwendung einschlägiger abstrakter Regeln (BImSchG und zugehörige Durchführungsverordnungen) auf einen konkreten Fall. »Vollzug « bedeutet auf dieser Ebene - neben der Befolgung verfahrensrechtlicher Bestimmungen (wie Einhaltung von Fristen, Beteiligung der in ihren Belangen betroffenen Behörden und sonstigen Stellen u.ä.) ${ }^{11}-$ die inhaltliche Festlegung der Betreiberpflichten durch Auflagen und Bedingungen. $\mathrm{Ob}$ der Betreiber die Auflagen und Bedingungen auch tatsächlich befolgt, ist nach dieser Sicht nicht mehr Frage einer einzelfallbezogenen Normanwendung, sondern Frage einer zweiten, zeitlich notwendig nachgelagerten Durchsetzung vor Ort der in der ersten Stufe getroffenen gesetzesvollziehenden Entscheidung. ${ }^{12}$ Die maßgebliche Frage lautet also, ob bzw. inwieweit den im Genehmigungsbescheid festgesetzten Anforderungen im Ergebnis auch tatsächlich entsprochen wird. Eine große Zahl von Auflagen, die theoretisch eine vorbildliche $\gg$ Umsetzung « des BImSchG darstellen mögen, erfordert hinsichtlich ihres faktischen Vollzugs einen hohen Überwachungsaufwand der zuständigen Behörden. Auf der anderen Seite kann eine geringe Zahl von Auflagen Ausdruck einer den gesetzlichen Rahmen nicht ausschöpfenden Genehmigungspraxis sein. Sie dürfte aber den »Vorteil« eines weniger kontrollaufwendigen Folgevollzugs haben. Angesichts der begrenzten Kontrollkapazität der Behörden ist es nicht von vornherein ausgemacht, welche der beiden Varianten am Ende, gemessen an der tatsächlich erreichten Umweltverträglichkeit eines Anlagenbetriebs, die erfolgreichere ist. In bezug auf diese Unterscheidung ist es die erste Vollzugsstufe, auf die sich hier die Frage nach den Auswirkungen unterschiedlicher behördlicher Genehmigungszuständigkeiten richtet.

10 Eine Differenzierung, die üblicherweise in den einschlägigen Implementationsstudien so nicht vorgenommen wird.

11 Eine übersichtliche Darstellung der für die Vollzugspraxis wichtigsten Bestimmungen des BImSchG einschließlich zugehöriger Verordnungen und allgemeiner Verwaltungsvorschriften enthält Dose 1997, S. $171 \mathrm{ff}$.

12 Insoweit reflektiert die Differenzierung des Gesetzesvollzugs in zwei »Stufen« auch die von Lübbe-Wolff 1993, S. 218, getroffene Feststellung: »Anlagen werden mit standardisierten Auflagenkatalogen genehmigt, aber entweder überhaupt nicht oder ohne wirkliche, detaillierte Überprüfung behördlich abgenommen.« 


\section{Anlage der Untersuchung}

\section{Erhebungsmethode}

Die vergleichende Analyse nord- und süddeutscher Genehmigungspraxis stützt sich im wesentlichen auf die Auswertung der Akten von insgesamt 125 abgeschlossenen vereinfachten Genehmigungsverfahren. ${ }^{13}$ Davon stammen 67 Genehmigungsakten von den beiden baden-württembergischen Landratsämtern [im folgenden verkürzt LRA-1 und LRA-2] und 58 Genehmigungsakten von dem niedersächsischen staatlichen Gewerbeaufsichtsamt [im folgenden GAA-N]. Die Auswahl der Behörden war durch Zugangsprobleme zum Feld bedingt (vgl. dazu näher unten Ziff. 2).

Die Auswertung der Akten erfolgte an Hand eines umfassenden, mehrteiligen Erhebungsbogens. ${ }^{14}$ Der überwiegende Teil der erhobenen Variablen betrifft die Kontakte zwischen Genehmigungsbehörde und Antragsteller. Außerdem wurden die Interaktionen der beiden Hauptakteure mit drei (bzw. vier) weiteren, ebenfalls maßgeblichen Verfahrensbeteiligten erfaßt. Im einzelnen handelt es sich dabei um das in der Regel angehörte Bauamt und - in Baden-Württemberg - das stets mitwirkende Gewerbeaufsichtsamt [GAA] sowie um die fallweise beteiligten Gemeinden als Trägerinnen der kommunalen Entwicklungsplanung und herangezogenen (unabhängigen) fachtechnischen Gutachter.

Die erhobenen Variablen lagen zum kleineren Teil in den Akten bereits in standardisierter, teils quantitativer, teils qualitativer Form vor - wie Zeitangaben, Anzahl der (dokumentierten) Kontakte zwischen einzelnen Verfahrensbeteiligten, Aufgabenbereich der beigezogenen Fachbehörden u.ä. Der übrige, größere Teil bezieht sich jedoch auf nicht-standardisierte, qualitative Merkmale, deren Operationalisierung durch ein entsprechendes Code-Buch vorbereitet wurde. ${ }^{15}$ Die (kategoriale) Operationalisierung der

13 Da die Untersuchung ausschließlich vereinfachte Verfahren i.S. von § 19 BImSchG zum Gegenstand hat, unterbleibt im weiteren die besondere Kennzeichnung als »vereinfacht $\ll$.

14 Von einer Wiedergabe des Erhebungsbogens wird hier wegen seines Umfangs (49 Seiten) abgesehen. Im übrigen gehen die erhobenen Daten (Variablen) aus der ausführlichen Darlegung der Untersuchungsergebnisse in Kap. III hervor.

15 Zur Validierung einer ersten Fassung der Erhebungsbögen und des zugehörigen Code-Buchs wurde ein Pretest durchgeführt. Dazu wurden für das niedersächsische Modell rd. 10 Akten abgeschlossener Genehmigungsverfahren des GAA-N ausgewertet. Für das baden-württembergische Modell konnte der Pretest nur in eingeschränkter Form durchgeführt werden, da zu diesem Zeitpunkt noch nicht die Akten 
qualitativen Merkmale war Voraussetzung für die spätere quantitative Auswertung der Daten als Grundlage des vorliegenden Modellvergleichs. ${ }^{16}$

Wie jede Methode erlaubt auch die Aktenanalyse nur einen spezifischen Zugang zum Untersuchungsgegenstand. Akten enthalten eine »Realität eigener Art: Die zum Zwecke der Rechtfertigung von Entscheidungen und ihrer rechtlichen Absicherung produzierte Version eines Entscheidungsablaufs « ${ }^{17}$ D.h. die aktenmäßige Wiedergabe von Vorgängen verfährt selektiv. Weder dokumentieren Akten alles, was sich zwischen den Beteiligten tatsächlich abgespielt hat, noch sind sie frei von »positionsgebundenen Strategien « der Akteure. ${ }^{18}$ Andererseits sind beim Anlegen und Führen von Akten Regeln einzuhalten, die es erlauben, den Verlauf des Entscheidungsprozesses aus der Sicht des »außenstehenden Dritten « (was nicht gleichbedeutend ist mit der Übernahme der Behördenversion) nachzuzeichnen und Verbindungslinien zwischen den Verfahrensbeteiligten herauszuarbeiten: $»$ Wer den Vorgang veranlaßt hat, wer der jeweils nächste Bearbeiter war, wann Terminierungen erfolgt sind «, wer wann welche Sachverhaltsdarstellungen und Rechtsansichten vorgetragen hat und welche Reaktionen darauf erfolgten bzw. welche Ergebnisse erzielt wurden, aber auch »welche Teilschritte die Akte belegt « und welche offensichtlich nicht. ${ }^{19}$

Zusammengefaßt zeichnet sich die Aktenanalyse gegenüber anderen Methoden dadurch aus, daß die dokumentierten Vorgänge Handlungen repräsentieren und nicht - wie etwa die (alleinige) Befragung der Verfahrensbeteiligten - nachträgliche Äußerungen über bestimmte Vorgänge. Anders als Befragungsergebnisse, denen das Problem einer doppelten Selektivität anhaftet, ${ }^{20}$ stellen die aktenmäßig festgehaltenen und für alle Beteiligten rechtlich verbindlichen Ergebnisse von Entscheidungsprozessen (z.B. Genehmigungsbescheide mit Nebenbestimmungen) einen »objektiv« gegebenen Maßstab zur Verfügung, der die Gültigkeit und Zuverlässigkeit gerade

der Genehmigungsbehörden (des LRA-1 bzw. des LRA-2) zugänglich waren, sondern lediglich die beim (für das LRA-2) zuständigen GAA vorhandenen, den Gesamtverlauf nur selektiv dokumentierenden Akten von (ebenfalls etwa 10) einschlägigen Genehmigungsverfahren zur Verfügung standen.

16 Zur Unterscheidung zwischen der Erhebung quantitativer oder qualitativer Daten (Merkmalen) einerseits und quantitativer oder qualitativer Datenauswertung am Beispiel der Verwaltungsforschung s. näher Derlien 1987 und ders. 1982.

17 Blankenburg 1975, S. 195. Vgl. zur Selektivität von (Verwaltungs-)Akten auch Hellstern 1984, insbes. S. 201-206.

18 Seibert 1981, S. 34. Im Einzelfall werden solche Strategien sogar ausdrücklich über Aktenvermerke festgehalten.

19 Seibert 1981, S. 33.

20 Es handelt sich dabei zum einen um die Selektivität der Erinnerung an einzelne Vorgänge, zum anderen um die Selektivität, mit der der Befragte auf die Interviewsituation i.d.R. reagiert. Zu letzterer vgl. etwa die anschaulichen Beispiele bei Hucke/ Wollmann 1980, S. 222-225. 
auch einer vergleichenden Analyse wie der vorliegenden zu erhöhen vermag.

Lediglich ergänzend zur Auswertung der Akten wurden mit den Behördenleitern und den zuständigen Sachbearbeitern vorstrukturierte Gespräche (Experteninterviews) geführt, die deren allgemeine Einschätzung der Handlungsmöglichkeiten ihrer Behörde gegenüber den antragstellenden Unternehmen zum Gegenstand hatten. ${ }^{21}$ Bei den Sachbearbeitern kamen Nachfragen zu einzelnen, von ihnen bearbeiteten Genehmigungsverfahren hinzu, wenn die aus der Aktenanalyse gewonnene Rekonstruktion des Verfahrensverlaufs Lücken oder Inkonsistenzen ergeben hatte.

\section{Auswahl der Genehmigungsbehörden}

Die hier gewählte Vergleichsgruppenanalyse ist eine bewährte Methode, um den vermuteten (ursächlichen) Einfluß des zugrundeliegenden Unterscheidungsmerkmals der beiden Gruppen, hier die unterschiedliche behördliche Genehmigungszuständigkeit (als unabhängige Variable), auf die Ausprägungen sonstiger Merkmale (als abhängige Variablen) der verfahrensförmigen Erarbeitung der Genehmigungsentscheidung zu ermitteln, hier also des Gesetzesvollzugs i.S. der oben (Kap. I) definierten ersten Stufe. Der Schluß, Veränderungen bei den abhängigen Variablen als Ursache der unabhängigen Variablen zuzurechnen, setzt voraus, daß sich die Verfahren beider Gruppen in allen übrigen Merkmalen (bzw. der Verteilung ihrer Ausprägungen), die - wie die Genehmigungszuständigkeit - als sog. Randbedingungen theoretisch ebenfalls den Gesetzesvollzug beeinflussen könnten, nicht unterscheiden (ceteris paribus-Klausel). Darunter fallen etwa auf Behördenseite die Zahl der Sachbearbeiter, auf die sich die untersuchten Genehmigungsverfahren verteilen, auf seiten des Antragstellers etwa die Art der beantragten Anlage oder die Größe seines Unternehmens (hierzu näher unten Ziff. 4).

Aus naheliegenden Gründen ist Konstanz sämtlicher Randbedingungen in der sozialwissenschaftlichen Forschung nicht herzustellen. Das hieße vollständige Identität in den Merkmalsausprägungen des Untersuchungsfelds. Dem steht schon grundsätzlich entgegen, daß Identifizierung und Kleinarbeitung von Merkmalen bereits Ergebnis einer theoretischen Konstruktion des Gegenstands sind. Insofern muß sich die Forschung von vorn-

21 Die wesentlichen Inhalte der Interviews wurde jeweils nach Abschluß aus dem Gedächtnis schriftlich festgehalten. Unter methodischen Gesichtspunkten waren sie jedoch nicht als für sich selbst stehendes Datenkorpus angelegt. Entsprechend dienten die aus den Interviews gewonnenen Informationen in erster Linie als zusätzliche Erkenntnisquelle bei der Interpretation der Ergebnisse der Aktenanalyse und der Entwicklung weiterführender Hypothesen. 
herein auf die Auswahl eines überschaubaren Kranzes von erfahrungsgemäß für einen Gegenstandsbereich typischen Merkmalsdimensionen beschränken. Aber auch eine solche begrenzte Kontrolle der Randbedingungen ist von sozialwissenschaftlichen Untersuchungen, die wie auch die vorliegende als Feldforschung angelegt sind, nur schwer zu erfüllen. Da das Interesse der Analyse spezifischen thematischen Handlungszusammenhängen in einschlägigen, bestehenden (»natürlichen«) Handlungsfeldern und ihren Akteuren gilt, stehen als Auswahleinheiten nur jeweils komplette Handlungsfelder (und nicht einzelne Elemente daraus) zur Verfügung. Die Chance, hinsichtlich der jeweiligen Randbedingungen möglichst ähnliche Handlungsfelder als Untersuchungseinheiten zu finden, ist um so größer, je ungehinderter der Zugang zum gesamten einschlägigen Untersuchungsfeld ist. Bei Zugangsrestriktionen, die gerade im Bereich der öffentlichen Verwaltung (immer noch) weit verbreitet sind, ${ }^{22}$ muß dagegen eine methodengeleitete Auswahl zurücktreten zugunsten der Nutzung von Forschungsmöglichkeiten dort, wo - wie im vorliegenden Fall - die genannten drei Genehmigungsbehörden (s.o. Ziff. 1) die Einsichtnahme in das gewünschte Aktenmaterial gestattet haben.

Nachteil einer durch den Zugang zum Untersuchungsfeld bestimmten Auswahl der beiden Vergleichsgruppen ist, daß bei ihnen mangels vorgängiger Kontrolle mit ungleichen Randbedingungen zu rechnen ist, die sich im Einzelfall auf den Verlauf des Verfahrens auswirken können. Die Prüfung solcher möglichen »intervenierenden « (vom Untersuchungsansatz her unerwünschten) Effekte muß bei der Datenanalyse gewissermaßen »nachgeholt« werden, um ermittelte Vollzugsunterschiede nicht fälschlicherweise der verschiedenartigen Genehmigungsorganisation zuzuschreiben (vgl. hierzu unten Ziff. 4).

\section{Auswahl der Verfahren}

Erhebungsgrundlage der Untersuchung bilden die bei den drei Genehmigungsbehörden jeweils geführten Akten derjenigen Verfahren, in denen die Genehmigung zwischen Anfang 1994 und Mitte 1996 erteilt worden war. Die gewählte zeitliche Eingrenzung des Erhebungszeitraums sollte nicht nur ein gewisses $\mathrm{Maß}$ an Aktualität der Untersuchungsergebnisse verbürgen. Sie war vor allem auch geboten, um eine weitgehende Konstanz der rechtlichen Rahmenbedingungen in einem Untersuchungsbereich zu sichern, der sich durch eine rege Novellierungspraxis zentraler bundesgesetzlicher Grundlagen (insbes. BImSchG, 4. BImSchV, 9. BImSchV), aber auch - für den vorliegenden Ländervergleich relevanter - landesgesetzlicher

22 Inzwischen unter zusätzlicher (legitimierender) Berufung auf das geltende Datenschutzrecht. 
Durchführungsbestimmungen (Verwaltungsverordnungen) auszeichnet. ${ }^{23}$ Da die Menge der im Erhebungszeitraum abgeschlossenen Verfahren geringer als erwartet war, ${ }^{24}$ konnte bzw. mußte bei allen drei Untersuchungsbehörden, also auch bei der vergleichsweise größten niedersächsischen Behörde, eine Vollerhebung durchgeführt werden, um für statistische Analysen hinreichend große Grundgesamtheiten in den beiden Vergleichsländern zu erhalten. Einen Überblick über die Anzahl der bei den drei Genehmigungsbehörden untersuchten Verfahren gibt Tab. 1.1.

Tab 1.1

Anzahl der untersuchten vereinfachten Genehmigungsverfahren

\begin{tabular}{|l|lc|}
\hline Niedersachsen & GAA-N & 58 \\
\hline Baden-Württemberg & LRA-1 & 43 \\
\hline Baden-Württemberg & LRA-2 & 24 \\
\hline Gesamt & & 125 \\
\hline
\end{tabular}

Tab. 1.2 schlüsselt die Verfahren nach dem Jahr der Genehmigungserteilung auf. Wegen der im Vergleich zum GAA-N bei den beiden LRÄ ohnehin schon geringeren Menge an Genehmigungsverfahren wurden beim LRA-1 zwei und beim LRA-2 vier Fälle noch mit einbezogen, in denen die Genehmigung in den letzten Monaten des Jahres 1993 erteilt worden war.

23 Zitierte rechtliche Bestimmungen beziehen sich - soweit nicht anders vermerkt jeweils auf die im Untersuchungszeitraum gültige Fassung: BImSchG vom 15.3.1974 in der Fassung der Bekanntmachung vom 14.5.1990, zuletzt geändert durch das Gesetz vom 27.9.1994; 4. BImSchV vom 24.7.1985, zuletzt geändert durch Verordnung vom 26.10.1993; 9. BImSchV vom 18.2.1977 in der Fassung der Bekanntmachung vom 29.5.1992, zuletzt geändert durch Verordnung vom 27.9.1994.

24 In den beiden baden-württembergischen Landratsämtern konnte die Zahl der in den fraglichen Jahren erteilten Genehmigungen entsprechenden amtsintern geführten Listen entnommen werden. Eine solche Statistik stand im niedersächsischen Gewerbeaufsichtsamt nicht zur Verfügung. Hier mußten die einschlägigen Akten an Hand von in der Registratur jahrgangsmäßig erstellten Aufzeichnungen identifiziert werden, die sämtliche ausgehenden Zahlungsaufforderungen der Behörde für ihre Amtshandlungen, darunter eben auch die für erteilte BImSchG-Genehmigungen, enthielten. 
Tab 1.2

Jahr der Genehmigungserteilung der untersuchten Verfahren

\begin{tabular}{|c|c|c|c|}
\cline { 2 - 4 } \multicolumn{1}{c|}{} & GAA-N & $L R A-1$ & LRA-2 \\
\hline \multirow{2}{*}{1993} & 0 & 2 & 4 \\
& $0,0 \%$ & $4,7 \%$ & $16,7 \%$ \\
\hline \multirow{2}{*}{1994} & 11 & 8 & 12 \\
& $19,0 \%$ & $18,6 \%$ & $50 \%$ \\
\hline \multirow{2}{*}{1995} & 27 & 20 & 3 \\
& $46,6 \%$ & $46,5 \%$ & $12,5 \%$ \\
\hline \multirow{2}{*}{1996} & 20 & 13 & 5 \\
& $34,5 \%$ & $30,2 \%$ & $20,8 \%$ \\
\hline \hline \multirow{2}{*}{ Gesamt } & 58 & 43 & 24 \\
& $100,1 \%$ & $100,0 \%$ & $100,0 \%$ \\
\hline
\end{tabular}

* Abweichungen von 100,0\% durch Rundungen bei den prozentualen Einzelhäufigkeiten.

Die Zusammensetzung der untersuchten Verfahren nach dem Jahr der Antragstellung zeigt Tab. 1.3.

Tab. 1.3

Jahr der Antragstellung der untersuchten Verfahren

\begin{tabular}{|c|c|c|c|}
\cline { 2 - 4 } \multicolumn{1}{c|}{} & GAA-N & LRA-1 & LRA-2 \\
\hline \multirow{2}{*}{1992} & 1 & 5 & 0 \\
& $1,7 \%$ & $11,6 \%$ & $0,0 \%$ \\
\hline \multirow{2}{*}{1993} & 5 & 4 & 11 \\
& $8,6 \%$ & $9,3 \%$ & $45,8 \%$ \\
\hline \multirow{2}{*}{1994} & 23 & 12 & 9 \\
& $39,7 \%$ & $27,9 \%$ & $37,5 \%$ \\
\hline \multirow{2}{*}{1995} & 23 & 15 & 4 \\
& $39,7 \%$ & $34,9 \%$ & $16,7 \%$ \\
\hline \multirow{2}{*}{1996} & 6 & 7 & 0 \\
& $10,3 \%$ & $16,3 \%$ & $0,0 \%$ \\
\hline \hline \multirow{2}{*}{ Gesamt } & 58 & 43 & 24 \\
& $100,0 \%$ & $100,0 \%$ & $100,0 \%$ \\
\hline
\end{tabular}




\section{Randbedingungen der Verfahren}

Wie oben bereits angesprochen (Ziff. 2), konnte bei der Auswahl der Genehmigungsbehörden das Erfordernis gleicher Randbedingungen der untersuchten Genehmigungsverfahren nicht hinreichend berücksichtigt werden. Solche unterschiedlichen Randbedingungen, die unabhängig vom jeweiligen Genehmigungsmodell auf den Verfahrensverlauf einwirken könnten, können einmal auf seiten der Genehmigungsbehörde und - in Baden-Württemberg - des jeweils zugeordneten GAA (4.1), zum anderen auf seiten der Antragsteller bzw. der antragstellenden Unternehmen vorliegen (4.2).

\subsection{Behördliche Randbedingungen}

Als mögliche Einflußvariable auf den Untersuchungsgegenstand kommt bei der Genehmigungsbehörde vor allem die Anzahl der Sachbearbeiter in Frage, die mit der Durchführung der untersuchten Genehmigungsverfahren betraut waren. Je größer die Gruppe der Mitarbeiter ist, auf die sich die Verfahren verteilen, desto weniger können individuelle Besonderheiten des Arbeitsstils, der fachlichen Qualifikation, tätigkeitsbezogener Werthaltungen und Einstellungen u.ä. den behördlichen Verfahrensablauf prägen. Darüber hinaus erlaubt ein höherer Personalbestand ein größeres Maß an fachspezifischer Ausdifferenzierung der Aufgabenbereiche, mit denen der einzelne Mitarbeiter betraut ist. Ein fachlich begrenzteres Arbeitsfeld begünstigt den Erwerb zusätzlichen einschlägigen theoretischen und praktischen Spezialwissens und demzufolge eine Steigerung der fachlichen Kompetenzen.

Die jeweilige Anzahl der im Untersuchungszeitraum beteiligten Sachbearbeiter in den drei Genehmigungsbehörden zeigt Tab. 2.1 (Kategorie b). Bezieht man die Mitarbeiterzahl auf die jeweils zugehörige Gesamtheit der Genehmigungsverfahren (Kategorie a), ergibt sich die Zahl der im Durchschnitt je Bearbeiter erledigten Verfahren (Kategorie c). Steigender Durchschnittswert indiziert eine höhere Konzentration der Genehmigungen auf einzelne Sachbearbeiter. Damit verringert sich die Möglichkeit einer fachlichen Spezialisierung, und es steigt die Möglichkeit, daß (außerfachliche) individuelle Besonderheiten bei der Verfahrensdurchführung an Gewicht gewinnen.

Wie aus der Tabelle hervorgeht, waren bei der niedersächsischen Genehmigungsbehörde vergleichsweise erheblich mehr Sachbearbeiter mit den Genehmigungen befaßt als bei den beiden baden-württembergischen Ämtern, wo sich diese auf nur wenige Mitarbeiter konzentrierten, beim LRA2 sogar nur auf eine einzige Person. Außerdem verteilen sich die Verfahren 
beim GAA-N gleichmäßiger auf die einzelnen Sachbearbeiter als beim LRA- 1.25

Tab. 2.1

Anzahl der beteiligten Sachbearbeiter bei den Genehmigungsbehörden

\begin{tabular}{|l|c|c|c|}
\cline { 2 - 4 } \multicolumn{1}{c|}{} & GAA-N & LRA-I & LRA-2 \\
\hline a) Zahl der Genehmigungsverfahren & 58 & 43 & 24 \\
\hline b) Zahl der Sachbearbeiter & 25 & 5 & 1 \\
\hline c) Verfahren pro Sachbearbeiter & 2,3 & 8,6 & 24,0 \\
\hline
\end{tabular}

Die unterschiedliche personelle Situation im Hinblick auf den Grad der fachlichen Spezialisierung und Individualisierung (im hier verstandenen Sinne) ist allerdings nicht unabhängig von der jeweiligen Genehmigungsorganisation. Die baden-württembergische Lösung einer dezentralen Ansiediung der Genehmigungszuständigkeii auf der unteren Verwaltungsebene schafft zwangsläufig kleinere Verwaltungseinheiten als die niedersächsische Lösung einer Zentralisierung auf wenige staatliche Gewerbeaufsichtsämter. Der Faktor Personal der Genehmigungsbehörde ist also eine Variable des Genehmigungsmodells und keine davon unabhängige Randbedingung. Der »Ein-Mann-Betrieb « des LRA-2, der mit einem vergleichsweise kleinen Zuschnitt des regionalen Zuständigkeitsbereichs dieses Amts korrespondiert, dürfte allerdings nicht typisch für die dezentrale Variante sein. ${ }^{26}$

Eine vom Genehmigungsmodell tatsächlich unabhängige Randbedingung ist dagegen die personelle Ausstattung der an den Genehmigungen als wichtigster Fachbehörde beteiligten Gewerbeaufsichtsämter. ${ }^{27}$ Dies sind in Baden-Württemberg die für LRA-1 und LRA-2 jeweils zuständigen GAA-

25 Während beim GAA-N die höchste Zahl der von ein und derselben Person bearbeiteten Fälle 12,1\% des Gesamtaufkommens der Genehmigungen ausmacht, sind es $34,9 \%$ beim LRA-1. Die ungleiche Streuung der Fallzahlen über die einzelnen Mitarbeiter spiegelt sich auch in der signifikanten Differenz der Varianzen $(F=9,22>$ $\left.\mathrm{F}_{(0,999 ; 4,24)}=6,59\right)$. Vgl. im einzelnen Tab. 2.1-A im Anhang.

26 Im Zuge der Datenerhebung stellten sich noch weitere atypische Randbedingungen beim LRA-2 heraus, so daß es sich im Ergebnis empfahl, dieses Amt nicht systematisch in den vorliegenden Modellvergleich einzubeziehen (vgl. dazu unten Kap. III mit Anm. 53).

27 In Baden-Württemberg wurde das dezentrale Modell der Genehmigungszuständigkeit (für vereinfachte Verfahren) bei der allgemeinen unteren Verwaltung im Jahre 
1 und GAA-2, in Niedersachsen ist es das zugleich als Fachbehörde fungierende GAA-N. Tab. 2.2 stellt die personellen Verhältnisse bei diesen drei Behörden dar.

\section{Tab. 2.2}

Anzahl der beteiligten Sachbearbeiter bei den zuständigen Gewerbeaufsichtsämtern

\begin{tabular}{|l|c|c|c|}
\cline { 2 - 4 } \multicolumn{1}{c|}{} & GAA-N & GAA-1 & GAA-2 \\
\hline Zahl der Genehmigungsverfahren & 58 & 43 & 24 \\
\hline Zahl der Sachbearbeiter & 25 & 19 & 14 \\
\hline Verfahren pro Sachbearbeiter & 2,3 & 2,3 & 1,7 \\
\hline
\end{tabular}

Anders als bei den Genehmigungsbehörden ergibt sich für die immissionsschutzrechtlichen Fachbehörden eine weitgehend ähnliche Situation. Das gilt sowohl für die durchschnittlich auf einen Bearbeiter entfallenden Genehmigungen als auch für das $\mathrm{Maß}$ der Gleichverteilung auf die einzelnen Personen. ${ }^{28}$

1985, also knapp eine Dekade nach Inkrafttreten des BImSchG, eingeführt. Die vom damaligen Leiter des größten Regierungspräsidiums des Landes M. Bulling initiierte Reform, die zu Lasten der bis dahin wie in Niedersachsen über die Genehmigungskompetenz verfügenden staatlichen Gewerbeaufsichtsämter ging, sollte der offiziellen Begründung nach hauptsächlich für eine größere »Bürgernähe« der Verwaltung sorgen. Im Untersuchungszeitraum (1993 bis 1996) war die neue Regelung nahezu 10 Jahre und damit in etwa ebenso lange wie die frühere alte praktiziert worden. Dies ist einerseits eine hinreichende Zeitspanne, um Übergangsprobleme zu bewältigen und an die veränderte Situation angepaßte Routinen im Verwaltungsalltag $\mathrm{zu}$ entwickeln. Andererseits ist nicht auszuschließen (und muß hier offenbleiben), daß trotz der inzwischen verstrichenen Frist die vor Einbuße der Genehmigungskompetenz vorhandene größere Autonomie der Behörde Spuren in deren »Gedächtnis« hinterlassen hat. Aus dieser Quelle könnten sich u.U. Bemühungen speisen, die verlorene Entscheidungsbefugnis durch betontes »Ausspielen« der verbliebenen fachtechnischen Autorität zu kompensieren.

28 Der paarweise Vergleich der Varianzen ergibt keine signifikanten Differenzen (GAA-1 zu GAA-N: $\mathrm{F}=1,34<\mathrm{F}_{(0,95 ; 18,24)}=2,05$; GAA-1 zu GAA-2: $\mathrm{F}=3,13<$ $\mathrm{F}_{(0,995 ; 18,13)}=2,98 ;$ GAA-N zu GAA-2: $\left.\mathrm{F}=2,34<\mathrm{F}_{(0,95 ; 24,13)}=2,89\right)$. Das gleiche gilt für die Mittelwerte (GAA-1 zu GAA-N: $\mathrm{t}=0,12<\mathrm{t}_{(0,95 ; 42)}=1,68$; GAA-1 $\mathrm{zu}$ GAA-2: $\mathrm{t}=2,00<\mathrm{t}_{(0,975 ; 31)}=2,04 ;$ GAA-N zu GAA-2: $\left.\mathrm{t}=1,34<\mathrm{F}_{(0,95 ; 37)}=1,69\right)$. Vgl. im einzelnen Tab. 2.2-A im Anhang. 
Unterschiedliches Verhalten des niedersächsischen GAA-N und des baden-württembergischen GAA-1 (bzw. GAA-2) in den Genehmigungsverfahren läßt sich somit nicht auf die Personalausstattung zurückführen. Vielmehr dürfte die Ursache dafür in den modellbedingten unterschiedlichen Funktionen zu suchen sein.

Ebenfalls durch in erster Linie modellinhärente Bedingungen dürfte sich die unterschiedliche Präsenz von Vorgesetzten bei der Verfahrensdurchführung erklären. Tab. 2.3 zeigt die Häufigkeit, mit der die Sachbearbeiter der Genehmigungsbehörden ihre Vorgesetzten in das laufende Verfahren i.S. einer unmittelbaren Beteiligung an dessen Durchführung einschalteten oder Vorgesetzte sich auf Bitten oder Drängen des Antragstellers in das Verfahren »einmischten«. Bloße Verwaltungsroutinen wie das Abzeichnen von Schriftstücken, insbesondere des Genehmigungsbescheids, fallen hier also nicht darunter.

Tab. 2.3

Beteiligung von Vorgesetzten bei der Verfahrensdurchführung (Genehmigungsbehörden)

\begin{tabular}{|l|c|c|c|}
\cline { 2 - 4 } \multicolumn{1}{c|}{} & $G A A-N$ & $L R A-1$ & $L R A-2$ \\
\hline Ohne Vorgesetztenbeteiligung & 55 & 18 & 16 \\
& $94,8 \%$ & $41,9 \%$ & $66,7 \%$ \\
\hline Mit Vorgesetztenbeteiligung & 3 & 25 & 8 \\
& $5,2 \%$ & $58,1 \%$ & $33,3 \%$ \\
[darunter: Amtsleiter (GAA-N)/ & {$[-]$} & {$[9]$} & {$[5]$} \\
Dezernent (LRÄ)] & {$[-]$} & {$[20,9 \%]$} & {$[20,8 \%]$} \\
\hline \hline Verfahren gesamt & 58 & 43 & 24 \\
\hline \hline
\end{tabular}

Während beim GAA-N Vorgesetzte bei der Verfahrensdurchführung kaum eine Rolle spielten, wirkten sie beim LRA-1 in mehr als der Hälfte der Fälle mit, beim LRA-2 zu einem Drittel. Die Differenzen zwischen GAA-N einerseits und den beiden LRÄ andererseits sind statistisch hochsignifikant. ${ }^{29}$ Hinzu kommt, daß beim GAA-N die jeweiligen Vorgesetzten lediglich den

29 Die Testwerte lauten für den Vergleich zwischen GAA-N und LRA-1: chi $^{2}=34,36$ $>10,82$, für den Vergleich zwischen GAA-N und LRA-2: chi $^{2}=11,22$ (Yates' Korrektur) $>10,82$, jeweils $p=0,001$ und $\mathrm{df}=1$. - Die Anteilsunterschiede zwischen den beiden LRÄ sind auf dem untersten Niveau einer Irrtumswahrscheinlichkeit von $10 \%$ signifikant: $\mathrm{chi}^{2}=3,75>2,71, \mathrm{p}=0,1, \mathrm{df}=1$. 
Rang eines Abteilungsleiters innehatten. Dagegen war beim LRA-1 in mehr als einem Drittel (beim LRA-2 in mehr als der Hälfte) der einschlägigen Fälle der in der Behördenhierarchie höher angesiedelte (fachlich zuständige) Dezernent beteiligt, dessen Position mit der des Amtsleiters eines Gewerbeaufsichtsamts vergleichbar ist. Auf Anlässe und Folgen der Einschaltung von Vorgesetzten wird bei den Untersuchungsergebnissen zurückzukommen sein (Kap. III, Ziff. 3.1.4).

\subsection{Betriebliche Randbedingungen}

Auf betrieblicher Seite könnten sich die Ausprägungen vor allem folgender drei Faktoren auf den Gesetzesvollzug auswirken: die Art der zur Genehmigung anstehenden Anlage entsprechend der Klassifikation im Anhang der 4. BImSchV, die Größe des antragstellenden Unternehmens sowie die Art der beantragten Genehmigung im Hinblick auf die Errichtung einer neuen oder Änderung einer bereits bestehenden Anlage.

\subsubsection{Anlagenart}

Die zur Genehmigung anstehende Anlagenart nach der Klassifikation der 4. BImSchV könnte insofern den Vollzug beeinflussen, als etwa Anlagen zur Abfallagerung, - sortierung und -aufbereitung andere umweltschutzbezogene (fachtechnische) Probleme und ggf. auch andere genehmigungsrechtliche Fragen aufwerfen als etwa Anlagen zur Herstellung oder Verarbeitung von Pflanzenschutz- und Arzneimitteln oder Anlagen zur Wärmeerzeugung (wie Feuerungsanlagen und Verbrennungsmotoren). ${ }^{30}$

Die Verteilung der Verfahren auf die verschiedenen Anlagenarten zeigt Tab. 3. Danach besteht zwischen dem niedersächsischen GAA-N und dem baden-württembergischen LRA-1 die größte Differenz in der Sparte Chemie (Nr. 4 und 5 zusammengefaßt), die beim GAA-N mit zusammengenommen $41,4 \%$ der Verfahren zu Buche schlägt gegenüber einem nur knapp halb so großen Anteil beim LRA-1 von $20,9 \%,{ }^{31}$ was auf unterschiedliche

30 Zwar ist die Klassifizierung einer beantragten Anlage nach dem Anhang der 4. BImSchV verfahrensrechtlich Teil der behördlichen Genehmigungsentscheidung und insofern strikt genommen eine abhängige Variable. Da aber die Klassifikation an technisch weitgehend eindeutigen, leicht objektivierbaren Kriterien ausgerichtet ist, überläßt sie der Praxis kaum Interpretationsspielräume. Dies ergeben auch die Untersuchungsbefunde, wonach Dissense bei der Anlagenzuordnung selten auftraten und in diesen Fällen lediglich Nuancierungen und nicht die Grundkategorien betrafen.

31 Der Unterschied ist statistisch signifikant $\left(\right.$ chi $\left.^{2}=4,70>3,84, p=0,05, \mathrm{df}=1\right)$. 
gewerblich-industrielle Schwerpunkte in den Zuständigkeitsbereichen der beiden Genehmigungsbehörden hindeutet. Eine weitere auffällige Abweichung ist bei den Sportanlagen (Nr. 10) festzustellen, die nur beim LRA-1, und zwar mit rd. einem Sechstel der Verfahren, vorkommen. ${ }^{32}$ Ihr Fehlen beim GAA-N liegt darin begründet, daß Sportanlagen nach der niedersächsischen Zuständigkeitsverordnung zu den (wenigen) Ausnahmen gehören, bei denen die Kommunen (Landkreise bzw. kreisfreie Städte) und nicht die staatlichen Gewerbeaufsichtsämter Genehmigungsbehörde sind. Bei den übrigen Kategorien bewegen sich die Abweichungen zwischen den beiden Ämtern unterhalb der 10\%-Marke. An dieser Stelle sei bereits vorweggenommen, daß ein systematischer Einfluß der vorgefundenen unterschiedlichen Anteile an Verfahren mit (kürzelhaft gesprochen) chemischen Anlagen bzw. Sportanlagen auf den Verfahrensablauf nicht festgestellt werden konnte. Dies dürfte auf die im übrigen ähnliche, insgesamt breite Streuung der Verfahren über die verschiedenen Anlagenklassen zurückzuführen sein.

Wirft man noch einen Blick auf die Anlagenarten der Verfahren beim LRA-2, zeigt sich, daß hier der chemische Bereich, der beim GAA-N, aber auch, wenn auch mit einem geringeren Anteil, beim LRA-1 die jeweils am häufigsten vertretene Anlagenart stellt, völlig fehlt. Gut die Hälfte der Verfahren entfällt dort zu nahezu gleichen Anteilen auf die Bereiche Herstellung/Bearbeitung von Baustoffen (Nr. 2) und Abfallagerung, -sortierung und -aufbereitung (Nr. 8). ${ }^{33}$ Die Mehrheit dieser Anlagen dienten der Aufbereitung bzw. der Vorbereitung der Aufbereitung von Altstoffen zur Wiederverwertung (Recycling). Auf einen vereinfachten Nenner gebracht, kann man sagen, daß unter den drei Genehmigungsbehörden das GAA-N über das vergleichsweise am stärksten und das LRA-2 über das am wenigsten industriell geprägte Einzugsgebiet verfügt.

32 Auch diese Differenz ist statistisch signifikant $\left(\right.$ chi $^{2}=9,0$ [Yates' Korrektur] $>7,38$, $\mathrm{p}=0,005, \mathrm{df}=1)$.

33 Der geringe Anteil von Anlagen zur Abfallagerung, -sortierung und -aufbereitung beim GAA-N erklärt sich daraus, daß diese ab September 1993 der Genehmigungszuständigkeit der Bezirksregierung unterfielen. 
Tab. 3

Anlagenart der Genehmigungsverfahren (nach den Ziff. des Anhangs der 4. BImSchV)

\begin{tabular}{|c|c|c|c|}
\hline$G A A-N$ & $L R A-I$ & $L R A-2$ & Anlagen zur: [Ziffer 4, BImSchV] \\
\hline $\begin{array}{c}7 \\
12,1 \%\end{array}$ & $\begin{array}{c}2 \\
4,7 \%\end{array}$ & $\begin{array}{c}3 \\
12,5 \%\end{array}$ & $\begin{array}{l}\text { 1: Wärmeerzeugung (Feuerunganlagen, } \\
\text { Verbrennungsmotoren) } \\
{[1.2 \mathrm{a}, 1.2 \mathrm{~b}, 1.4,1.4 \mathrm{a}, 1.4 \mathrm{~b}, 1.8]}\end{array}$ \\
\hline $\begin{array}{c}12 \\
20,7 \%\end{array}$ & $\begin{array}{c}7 \\
16,3 \%\end{array}$ & $\begin{array}{c}7 \\
29,2 \%\end{array}$ & $\begin{array}{l}\text { 2: Herstellung/Bearbeitung von (nicht-metall.) Baustof- } \\
\text { fen (Gestein, Zement, Bitumen) } \\
{[2.1,2.2,2.13,2.14,2.15]}\end{array}$ \\
\hline $\begin{array}{c}6 \\
10,3 \%\end{array}$ & $\begin{array}{c}2 \\
4,7 \%\end{array}$ & $\begin{array}{c}4 \\
16,7 \%\end{array}$ & $\begin{array}{l}\text { 3: Herstellung/Bearbeitung (Schmelzen, Beschichten) } \\
\text { von Metallen } \\
{[3.4,3.8,3.9 \mathrm{a}, 3.9 \mathrm{~b}, 3.10,3.14]}\end{array}$ \\
\hline $\begin{array}{c}6 \\
10,3 \%\end{array}$ & - & - & $\begin{array}{l}\text { 4: Herstellung/Verarbeitung von Pflanzenschutz- und } \\
\text { Arzneimitteln (Chemie I) } \\
{[4.2,4.3 \mathrm{~b}, 4.3 \mathrm{c}]}\end{array}$ \\
\hline $\begin{array}{c}18 \\
31,0 \%\end{array}$ & $\begin{array}{c}9 \\
20,9 \%\end{array}$ & - & $\begin{array}{l}\text { 5: Herstellung/Behandlung von Gegenständen unter Ver- } \\
\text { wendung organischer Lösungsmittel (Chemie II) } \\
{[4.8,4.10,5.1 \mathrm{a}, 5.1 \mathrm{~b}, 5.1 \mathrm{c}, 5.5,5.8,5.10,10.7,10.8 \text {, }} \\
\text { 10.21, 10.23] }\end{array}$ \\
\hline$\stackrel{1}{1,7 \%}$ & $2,3 \%$ & - & $\begin{array}{l}\text { 6: Papierherstellung } \\
{[6.2]}\end{array}$ \\
\hline $\begin{array}{c}2 \\
3,4 \%\end{array}$ & $\stackrel{5}{11,6 \%}$ & - & $\begin{array}{l}\text { 7: Herstellung/Bearbeitung von Nahrungs- und } \\
\text { Futtermitteln } \\
{[7.4,7.5,7.21,7.27,7.29]}\end{array}$ \\
\hline $\begin{array}{c}2 \\
3,4 \%\end{array}$ & $\begin{array}{l}4 \\
9,3 \%\end{array}$ & $\begin{array}{c}6 \\
25,0 \%\end{array}$ & $\begin{array}{l}\text { 8: Abfallagerung, -sortierung und -aufbereitung } \\
{[8.4, \mathrm{a}, 8.4 \mathrm{~b}, 8.9,8.11]}\end{array}$ \\
\hline $\begin{array}{l}4 \\
6,9 \%\end{array}$ & $\begin{array}{c}6 \\
14,0 \%\end{array}$ & $\begin{array}{c}3 \\
12,5 \%\end{array}$ & $\begin{array}{l}\text { 9: Lagerung von brennbaren/giftigen Stoffen } \\
\text { (Gase, Mineralöle) } \\
{[9.1 b, 9.2 a, 9.34,9.35,10.25]}\end{array}$ \\
\hline- & $\begin{array}{c}7 \\
16,3 \%\end{array}$ & $\begin{array}{l}1 \\
4,2 \%\end{array}$ & $\begin{array}{l}\text { 10: Sportanlagen (Motor-, Schießsport) } \\
{[10.17,10.18]}\end{array}$ \\
\hline $\begin{array}{c}\mathbf{5 8} \\
99,8 \%\end{array}$ & $\begin{array}{c}\mathbf{4 3} \\
100,1 \%\end{array}$ & $\begin{array}{c}24 \\
100,1 \%\end{array}$ & Verfahren gesamt* \\
\hline
\end{tabular}

* Abweichungen von 100,0\% durch Rundungen bei den prozentualen Einzelhäufigkeiten. 


\subsubsection{Unternehmensgröße}

Wirtschaftskraft und personelle Ressourcen des antragstellenden Unternehmens sind ein weiterer Faktor, der auf den Verfahrensverlauf einwirken könnte. Je besser ein Unternehmen ökonomisch und personell ausgestattet ist, so die Annahme, um so eher wird es in der Lage sein, seinen Interessen gegenüber den Behörden Nachdruck zu verleihen. Die Verfügung über einschlägiges fachtechnisches Spezialwissen und ein versiertes, oft auch juristisch qualifiziertes Management ${ }^{34}$ machen solche Unternehmen zu mindestens gleichwertigen, vielfach der Verwaltung auch überlegenen Verhandlungspartnern. Hinzu kommt ihr ökonomisches Gewicht als Arbeitsplätze schaffende bzw. sichernde Arbeitgeber, deren Investitionsbereitschaft spätestens seit der in den 90er Jahren einsetzenden »Wirtschaftsstandort Deutschland «-Debatte ${ }^{35}$ faktisch zu einem staatlich schutzwürdigen öffentlichen Interesse avanciert ist.

Die nachfolgende Klassifizierung der die genehmigungsbedürftigen Anlagen betreibenden Unternehmen bezieht sich ausschließlich auf den hier interessierenden Zusammenhang ihres möglicherweise verfahrenswirksamen Potentials, das näherungsweise als »Unternehmensgröße« operationalisiert ist. Dabei wird zudem nur eine grobe dreistufige, relational (nicht absolut) zu verstehende Einteilung in »größere«, »mittlere« ünd »kleinere« Unternehmen zugrunde gelegt. Für die jeweilige Zuordnung wurden als Kriterien die Rechtsform, überregionale Präsenz, Investitionskosten für die beantragte Anlage bzw. deren Änderung und Zahl der Beschäftigten herangezogen. Rechtsform und Investitionskosten konnten durchgehend den Akten entnommen werden, Angaben über den Personalbestand fehlten entweder überhaupt oder umfaßten nur die an der Anlage arbeitenden Arbeitnehmer. ${ }^{36}$ Die überregionale Präsenz war teilweise durch die namentlich allgemeine Bekanntheit eines Unternehmens offenkundig, in den übrigen Fällen ergab sich die mögliche überlokale Bedeutung eines Unternehmens teils aus den Akten, teils aus entsprechenden Hinweisen der Sachbearbeiter.

Der Gruppe der »größeren« Unternehmen wurden alle als Aktiengesellschaft verfaßten Unternehmen sowie Firmen zugerechnet, die in anderen Rechtsformen wie der der $\mathrm{GmbH}, \mathrm{GmbH} \& \mathrm{Co} . \mathrm{KG}$ und $\mathrm{KG}$ organisiert und überregional vertreten sind. ${ }^{37}$ Den Gegenpol der »kleineren« Unternehmen

34 Großunternehmen haben im übrigen vielfach eine eigene Abteilung für die Durchführung von Genehmigungsverfahren errichtet.

35 Vgl. hierzu näher unten Kap. III, Ziff. 4.1 mit Anm. 298.

36 Die Genehmigungsakten werden anlagen- und nicht betriebsbezogen geführt.

37 Die letztgenannten Rechtsformen waren ursprünglich für mittelständische und kleinere Betriebe zur Kapitalbeschaffung konzipiert. Sie werden häufig aber auch von über die mittelständische Betriebsgröße hinausgewachsenen Unternehmen aus verschiedenen Gründen (wie Besteuerungsgrundlagen oder Publizitätspflichten) gewählt. 
bilden Kapital- und Personengesellschaften mit einer geringen Zahl von Mitarbeitern und einem auf den lokalen Nahbereich begrenzten Betätigungsfeld sowie nicht als Gesellschaft verfaßte Gewerbetreibende. Alle übrigen Unternehmen fallen entsprechend in die Gruppe der »mittleren« Unternehmen, die zur Wahrung der Homogenität i.S.d. Trennschärfe der beiden Extremklassen eine Art Auffangkategorie darstellt und von daher ein breiteres Spektrum unterschiedlicher Unternehmensgrößen umfaßt.

Die Verteilung der Genehmigungsverfahren auf die so definierten GröBenklassen der antragstellenden Unternehmen zeigt Tab. 4. Danach wurde beim GAA-N die Hälfte (50,0\%) der Genehmigungsverfahren von größeren Firmen geführt, kleinere Firmen kommen nur auf einen Anteil von weniger als einem Zehntel (6,9\%). Beim LRA-1 stellen demgegenüber die größeren Firmen nur ein gutes Viertel $(27,9 \%)$ und die kleineren nahezu ein Drittel $(32,6 \%)$ der Verfahren. Vergleichbare Werte ergeben sich beim LRA-2. ${ }^{38}$ Die gegenläufige Verteilung der Unternehmen zugunsten der größeren im Norden und der kleineren im Süden bei ähnlichen Anteilen bei den mittleren ist statistisch signifikant. ${ }^{39}$

Tab. 4

Unternehmensgröße der Antragsteller

\begin{tabular}{|l|c|c|c|}
\cline { 2 - 4 } \multicolumn{1}{c|}{} & GAA-N & LRA-1 & LRA-2 \\
\hline \multirow{2}{*}{ Größere Firmen } & 29 & 12 & 6 \\
& $50,0 \%$ & $27,9 \%$ & $25,0 \%$ \\
\hline \multirow{2}{*}{ Mittlere Firmen } & 25 & 17 & 12 \\
& $43,1 \%$ & $39,5 \%$ & $50,0 \%$ \\
\hline \multirow{2}{*}{ Kleinere Firmen } & 4 & 14 & 6 \\
\hline \hline \multirow{2}{*}{ Verfahren gesamt } & $6,9 \%$ & $32,6 \%$ & $25,0 \%$ \\
\hline \hline
\end{tabular}

38 Die Verteilungsunterschiede zwischen LRA-1 und LRA-2 sind nicht signifikant $\left(\mathrm{chi}^{2}=0,74<4,61\right.$ für $\mathrm{p}=0,1$ und $\left.\mathrm{df}=2\right)$.

39 Die Testwerte lauten für den Vergleich zwischen GAA-N und LRA-1: chi $^{2}=12,10$ $>10,6, p=0,005$, für den Vergleich zwischen GAA-N und LRA-2: chi $^{2}=6,80>$ $5,99, \mathrm{p}=0,05$, jeweils $\mathrm{df}=2$. 


\subsubsection{Externe Beratung}

Ein weiterer Faktor, der sich auf das Verfahren auswirken könnte, ist die Unterstützung des Antragstellers durch externen Sachverstand ${ }^{40}$ bei der Antragsbearbeitung und/oder der Verfahrensdurchführung. Die »eingekaufte« Kompetenz erhöht potentiell die Durchsetzungsfähigkeit des Unternehmens gegenüber den Behörden. Diesen ist ihrerseits durchaus an einem möglichst kompetenten Antragsteller gelegen, der sie von eigener Beratungstätigkeit entlastet, um den Preis allerdings, ggf. auf mehr Widerstand gegen ihre Anforderungen zu stoßen.

Tab. 5

Beratung des Antragstellers durch externen Sachverstand

\begin{tabular}{|l|c|c|c|}
\cline { 2 - 4 } \multicolumn{1}{c|}{} & GAA-N & LRA-1 & LRA-2 \\
\hline \hline a) Ohne externe Beratung & 34 & 21 & 14 \\
& $58,6 \%$ & $48,8 \%$ & $58,3 \%$ \\
\hline $\begin{array}{l}\text { b) Externe Beratung: } \\
\text { nur Antragsbearbeitung }\end{array}$ & 15 & 11 & 6 \\
\hline $\begin{array}{l}\text { c) Externe Beratung: } \\
\text { nur Verfahrensdurchführung }\end{array}$ & $25,9 \%$ & $25,6 \%$ & $25,0 \%$ \\
\hline d) Externe Beratung: & 1 & 1 & - \\
Antrag und Verfahren & $1,7 \%$ & $2,3 \%$ & $0,0 \%$ \\
\hline \hline Verfahren gesamt & 8 & 10 & 4 \\
\hline
\end{tabular}

Tab. 5 zeigt die Anteile der Unternehmen, die in der einen oder anderen Form professionelle Fremdhilfe in Anspruch genommen haben. Im großen und ganzen sahen sich alle drei Genehmigungsbehörden einer ähnlichen Situation gegenüber. Jeweils rd. ein Viertel der Antragsteller hat sich gezielt bei der Antragserarbeitung (Kategorie b) beraten lassen. Ein jeweils geringerer, aber noch ins Gewicht fallender Teil hat sich zusätzlich auch bei der Verfahrensdurchführung sachkundiger Unterstützung bedient (Kategorie d), wobei dieser Anteil zwischen 13,8\% beim GAA-N und 23,3\% beim LRA-1 streut. Eine erst bei der Verfahrensdurchführung beigezogene Bera-

40 Ausgenommen sind dabei Architekten, soweit sich ihre Mitwirkung auf die rein baurechtliche Genehmigung, die nach dem Konzentrationsprinzip des $\S 13$ BImSchG im Rahmen der immissionsschutzrechtlichen Genehmigung mit erteilt wird, beschränkt. 
tung (Kategorie c) bleibt die Ausnahme. Die Entscheidung für oder gegen die Inanspruchnahme externer Hilfe wird also anläßlich der Antragstellung, mithin frühzeitig, getroffen und im weiteren Verfahrensverlauf auch nicht mehr geändert.

Insgesamt haben beim GAA-N rd. $41 \%$ und beim LRA-1 rd. $51 \%$ der Unternehmen zur Erlangung der Genehmigung auf fremden Sachverstand zurückgegriffen (Kategorien b bis d), wobei die Differenz deutlich unterhalb des statistischen Signifikanzniveaus liegt. ${ }^{41}$ Umgekehrt besagt dieses Ergebnis, daß etwa die Hälfte der Unternehmen - mit gewissen Abweichungen nach oben oder unten bei den einzelnen Genehmigungsbehörden - entweder ihre eigene Kompetenz für ausreichend hielt oder sich aus finanziellen Erwägungen keinen externen Beistand leisten wollte bzw. konnte.

Zwischen externer Beratung und Unternehmensgröße ergibt sich bei allen drei Genehmigungsbehörden ein systematischer Zusammenhang in der (naheliegenden) Weise, daß größere Unternehmen dank ausreichender eigener Kompetenz am wenigsten auf fremden Sachverstand zurückgreifen und kleinere mangels genügenden eigenen Fachwissens am häufigsten. Eine mittlere Position nehmen die mittelgroßen Firmen ein mit einem Beratungsanteil von knapp $50 \% .{ }^{42} \mathrm{Ob}$ diese und insbesondere auch die kleineren Unternehmen durch den eingeholten externen Sachverstand ein den größeren vergleichbares Potential erreichten, die eigenen Interessen gegenüber den Behörden wirkungsvoll zu vertreten, ist fraglich.

Im Hinblick auf den hier im Vordergrund stehenden Modellvergleich ist die Unternehmensgröße unter dem Gesichtspunkt von Interesse, ob bzw. wieweit die unterschiedlichen Organisationsformen des Gesetzesvollzugs die jeweils zuständige Genehmigungsbehörde auch tatsächlich in die Lage versetzen, sich gegenüber kompetenten Antragstellern durchzusetzen. In dieser Hinsicht könnte man vermuten, daß das niedersächsische Modell, das dem als Genehmigungsbehörde fungierenden GAA einen größeren, kommunale Grenzen überschreitenden Zuständigkeitsbereich zuweist, die faktische Ausübung behördlicher Autonomie gegenüber dem Antragsteller eher unterstützt als das baden-württembergische Modell, das das zuständige LRA in die kommunalpolitischen Strukturen des zugehörigen Land-

41 Das gilt auch für den zwischen den beiden Werten liegenden Beratungsanteil von rd. $42 \%$ beim LRA-2. Die Testwerte lauten im einzelnen für den Vergleich zwischen GAA-N und LRA-1: chi $^{2}=0,94$, für den Vergleich zwischen LRA-1 und LRA-2: chi $^{2}=0,59$ jeweils $<2,71$, für $\mathrm{p}=0,1$ und $\mathrm{df}=1$.

42 Wegen der durch die Kreuztabellierung reduzierten absoluten Zellhäufigkeiten läßt sich der dargestellte Zusammenhang zwischen externer Beratung und Unternehmensgröße nur beim GAA-N im Hinblick auf den Vergleich der mittleren und gröBeren Firmen statistisch absichern: $\mathrm{chi}^{2}=5,80>5,02, \mathrm{p}=0,025$, df $=1$. Zu den Werten der Kreuztabellierungen im einzelnen vgl. Tab. 5-A im Anhang. 
kreises einbindet. ${ }^{43}$ Darüber hinaus könnten auch die unterschiedlichen Qualifikationen des Personals eine Rolle spielen, wobei es eine offene Frage ist, ob die niedersächsische fachtechnische Kompetenz oder die baden-württembergische verwaltungsrechtliche Kompetenz die jeweils geeignetere Grundlage für autonome Genehmigungsentscheidungen abgibt. ${ }^{44}$

\subsubsection{Genehmigungsart}

Ein vierter, von der Genehmigungsorganisation auf den ersten Blick unabhängiger Faktor ist die Art der nachgesuchten Genehmigung im Hinblick auf die Errichtung einer neuen Anlage oder die Änderung einer bereits bestehenden Anlage. Es geht also darum, ob es sich im konkreten Fall um eine Neu- oder um eine Änderungsgenehmigung handelt. ${ }^{45}$

43 Nach der baden-württembergischen Landkreisordnung werden der Landrat als Behördenleiter des LRA - unter Mitwirkung des Innenministeriums - wie auch die übrigen leitenden Beamten und Angestellten des Kreises vom Kreistag gewählt. Der Kreistag seinerseits wird dominiert von Angehörigen des Öffentlichen Dienstes, von denen wiederum ein hoher Anteil zugleich auch das Amt des Bürgermeisters einer Kreisgemeinde innehat. Diese Konstellation begünstigt eine faktische Abhängigkeit der Kreisverwaltung von kommunalpolitischen Kräfteverhältnissen und Interessenlagen. Vgl. hierzu näher Schneider 2000, insbes. S. 247f. u. S. $250 \mathrm{f}$. Vgl. auch Mayntz et al. 1978, S. 48, die im Falle kommunaler Genehmigungszuständigkeit eine größere Konzessionsbereitschaft zugunsten politischer Interessen als im Falle kommunalferner behördlicher Zuständigkeit (wie etwa hier der staatlichen Gewerbeaufsicht) sehen. Vg1. ferner auch Lübbe-Wolff 1990, S. 27, die in der kommunalpolitischen Einbindung der Genehmigungsbehörde zwar ebenfalls die Gefahr von einem strikten Gesetzesvollzug abträglichen Rücksichtnahmen insbes. auf Wirtschaftsförderungsinteressen der Gemeinden konstatiert, auf der anderen Seite aber auch auf die Möglichkeit für engagierte Bürger und die Lokalpresse hinweist, auf die Kommunalpolitik zugunsten des Umweltschutzes einzuwirken.

44 Insgesamt vermitteln die Untersuchungsbefunde den Eindruck, daß das baden-württembergische LRA eher als das niedersächsische GAA-N geneigt war, sich in seiner Verfahrensführung von der Größe eines Unternehmens beeindrucken zu lassen. Statistisch absichern ließ sich diese Tendenz aber - wegen der bei Untergruppenbildung zwangsläufig verringerten absoluten Fallzahlen - nur im Hinblick auf die Handhabung von Betriebskontrollen (s. unten Kap. III, Ziff. 2.4.5). In jedem Fall scheint die im Süden (formal) größere verwaltungsrechtliche Kompetenz des Personals in der Praxis keine die behördliche Autorität (zusätzlich) stützende Ressource zu sein.

45 Verfahrensrechtlich fällt die Zuordnung zur einen oder anderen Verfahrenskategorie zwar in die Entscheidungskompetenz der Genehmigungsbehörde. Insofern stellt sie strikt genommen eine abhängige Variable dar. Für die meisten Fallkonstellationen ist die rechtliche Abgrenzung von Neu- und Änderungsgenehmigung aber offenbar hinreichend eindeutig, so daß sich in dieser Hinsicht der behördlichen Praxis kein größerer Entscheidungsspielraum eröffnet. Hinzu kommt, daß die Zuordnung zur einen oder anderen Variante im vereinfachten Verfahren (zum Regelverfahren s. nachfolg. Anm.) keinen Einfluß auf den formellen Verfahrensablauf und den recht- 
Wenn auch beide Genehmigungsarten im vereinfachten Verfahren (im Unterschied zum »normalen « Verfahren $)^{46}$ grundsätzlich denselben verfahrensrechtlichen Regeln unterliegen, ${ }^{47}$ könnten sie in anderen verfahrensrelevanten Hinsichten unterschiedliche Fallkonstellationen repräsentieren. So gehen Änderungsgenehmigungen in vielen Fällen aus einer anders gelagerten betrieblichen Ausgangssituation hervor als Neugenehmigungen, ${ }^{48}$ was sich auch auf den weiteren Verfahrensverlauf auswirken könnte. Während die Neuerrichtung einer Anlage auf einer autonomen Entscheidung des Betreibers beruht, erfolgt die Änderung einer bestehenden Anlage häufig erst auf Druck der Genehmigungsbehörde, weil die Anlage den umweltschutzrechtlichen Anforderungen, meist wegen zwischenzeitlich herabgesetzter Grenzwerte oder des weiterentwickelten Stands der Technik, nicht mehr genügt und nur bei entsprechender Sanierung weiterbetrieben werden darf. Hinzu kommt, daß nicht wenige Änderungsgenehmigungsverfahren

lichen Rahmen des möglichen Genehmigungsinhalts hat. Von daher sind die Verfahrensbeteiligten in der Regel kaum daran interessiert, die Zuordnungskriterien zu problematisieren. Im Einzelfall (ein solcher Fall kam in der vorliegenden Untersuchung lediglich einmal vor) kann ein solches Interesse allerdings dann vorliegen, wenn es auf den nur bei Änderungsgenehmigungen gegebenen planungsrechtlichen Bestandsschutz ankommt. Eine von der Frage nach der Art der ersuchten Genehmigung grundsätzlich zu unterscheidende Frage ist die, ob eine Anlage oder deren Änderung überhaupt einer immissionsschutzrechtlichen Genehmigungspflicht unterliegt oder nicht. Insbesondere bei Änderungsgenehmigungen bietet das gesetzliche Kriterium der »wesentliche(n)« Änderung ( $§ 15$ Abs. 1 Satz 1 BImSchG) als Voraussetzung für das Genehmigungserfordernis Raum für unterschiedliche Bewertungen durch Genehmigungsbehörde und Betreiber. Der Bezug der vorliegenden Untersuchung auf abgeschlossene Genehmigungsverfahren bringt es mit sich, daß Streitfälle über die grundsätzliche Genehmigungspflicht einer Anlage bzw. Anlagenänderung immer zugunsten eines Genehmigungserfordernisses entschieden worden sind. Zu den Häufigkeiten derartiger Streitigkeiten s. im einzelnen unten Kap. III, Ziff. 3.1.2.

46 Im Regelverfahren hat die Genehmigungsbehörde das geplante Vorhaben bei Neuerrichtung öffentlich bekanntzumachen und den Antrag samt Unterlagen zur Einsicht auszulegen, um potentiell betroffenen Dritten Gelegenheit zu evtl. Einwendungen zu geben ( $\$ 10$ Abs. 3 BImSchG). Dagegen soll sie bei Änderungen einer bereits bestehenden (und in diesem Bestand genehmigten) Anlage unter bestimmten Bedingungen von der öffentlichen Bekanntmachung und Auslegung absehen (§ 15 Abs. 2 BImSchG).

47 Der nur bei Neuerrichtungen möglichen Beantragung einer Teilgenehmigung ( 88 BImSchG) entspricht die nur bei Änderungen einer bestehenden Anlage mögliche Beantragung der Zulassung vorzeitigen Beginns ( $\$ 15 \mathrm{a}$ BImSchG). Beide Varianten erlauben dem Betreiber im Ergebnis, bereits vor Erteilung der unabhängig davon in jedem Fall erforderlichen endgültigen Genehmigungserteilung mit der Umsetzung des Vorhabens zu beginnen. Zur Häufigkeit von zusätzlich zur (Haupt-) Genehmigung beantragter Teilgenehmigung bzw. Zulassung vorzeitigen Beginns in den hier untersuchten Verfahren s. unten Anm. 50.

48 Vgl. hierzu im einzelnen unten Kap. III, Ziff. 1.2. 
sich erst der behördlichen Aufdeckung einer ohne vorherige Genehmigung vorgenommenen Anlagenänderung verdanken. In beiden Fällen ist der Betreiber an einer mit Zeit- und vielfach auch finanziellem Aufwand verbundenen aktiven Mitwirkung am Verfahren eher desinteressiert, im ersten Fall, weil die nicht selten mit erheblichen Investitionskosten einhergehenden Sanierungsmaßnahmen - zumindest zum gegenwärtigen Zeitpunkt nicht geplant waren, im zweiten Fall, weil die Anlagenänderung bereits vollzogen ist (an die die Behörde möglicherweise zusätzliche Anforderungen stellt). Aus der vorliegenden Untersuchung ergibt sich allerdings, daß auch ungenehmigte Neuerrichtungen keine Ausnahmen sind.$^{49}$ Insofern relativiert sich der Unterschied in der betrieblichen Ausgangslage zwischen Neu- und Änderungsgenehmigungen. Grundsätzlich ist aber nicht von vornherein auszuschließen, daß ein Zusammenhang zwischen Entdekkungswahrscheinlichkeit ungenehmigter Anlagenerrichtung und -änderung einerseits und Genehmigungsorganisation andererseits besteht. Dann wäre das Aufkommen an Neu- und Änderungsgenehmigungen einer Behörde eher als abhängige denn als unabhängige Variable zu betrachten.

Die bei den untersuchten Genehmigungsbehörden vorgefundenen Anteile an Neu- und Änderungsgenehmigungen zeigt Tab. 6. Während beim LRA-2 auf jede Genehmigungsart genau die Hälfte der Verfahren entfällt, ging es beim GAA-N wie beim LRA-1 in der Mehrheit der Verfahren um Änderungsgenehmigungen $(72,4 \%$ bzw. $60,5 \%)$, entsprechend zum kleineren Teil um Neugenehmigungen $\left(27,6 \%\right.$ bzw. 39,5\%). ${ }^{50}$ Die anteiligen Differenzen bleiben dabei unterhalb statistischer Signifikanz. ${ }^{51}$ Vergleichbar sind auch die Anteile an (entdeckten) ungenehmigten Neuerrichtungen bzw. Anlagenänderungen (vgl. dazu näher unten Kap. III, Ziff. 1.2). Von daher ist die Wahrscheinlichkeit gering, daß Unterschiede im weiteren Verfahrensablauf zwischen diesen beiden Genehmigungsbehörden auf eine unterschiedliche Zusammensetzung der Genehmigungsart zurückzuführen sind. Dabei ist allerdings zu beachten, daß die Genehmigungsart mit der Unternehmensgröße insoweit kovariiert, als Änderungsgenehmigungen

49 Wie vorangehende Anm.

50 Zusätzlich zur (Haupt-) Genehmigung wurden bei beiden Ämtern zu ähnlichen Anteilen Anträge auf Teilgenehmigung (gem. § 8 BImSchG) bei Neugenehmigungen (GAA-N: 18,8\%, LRA-1: 23,5\%) und auf Zulassung vorzeitigen Beginns (gem. $\S 15$ a BImSchG) bei Änderungsgenehmigungen (GAA-N: 19,0\%, LRA-1: 15,4\%) gestellt. Die Prozentwerte beziehen sich jeweils auf die zugehörigen Grundgesamtheiten an Neu- bzw. Änderungsgenehmigungen.

$51 \mathrm{chi}^{2}=1,40<2,71$ für $\mathrm{p}=0,1$. Nicht signifikant sind auch die anteiligen Differenzen in der Verteilung auf Neu- und Änderungsgenehmigungen zwischen den beiden LRÄ $\left(\mathrm{chi}^{2}=0,67<2,71\right.$ für $\left.\mathrm{p}=0,1\right)$, signifikant dagegen die zwischen LRA-2 und GAA-N : $\left.\mathrm{chi}^{2}=3,78>2,71, \mathrm{p}=0,1\right)$. Alle Testwerte jeweils für $\mathrm{df}=1$. 
überproportional häufig größere (und mittlere) Unternehmen und Neugenehmigungen überproportional häufig kleinere Unternehmen betreffen. ${ }^{52}$

Tab. 6

Genehmigungsart der Verfahren

\begin{tabular}{|l|c|c|c|}
\cline { 2 - 4 } \multicolumn{1}{c|}{} & GAA-N & LRA-1 & LRA-2 \\
\hline \multirow{2}{*}{ Neugenehmigung } & 16 & 17 & 12 \\
& $27,6 \%$ & $39,5 \%$ & $50,0 \%$ \\
\hline Änderungsgenehmigung & 42 & 26 & 12 \\
& $72,4 \%$ & $60,5 \%$ & $50,0 \%$ \\
\hline \hline \multirow{2}{*}{ Verfahren gesamt } & 58 & 43 & 24 \\
& $100,0 \%$ & $100,0 \%$ & $100,0 \%$ \\
\hline
\end{tabular}

Betrachtet man die jeweilige Relation von Änderungs- und Neugenehmigungen bei den drei Genehmigungsbehörden im Vergleich, so verschiebt sich diese mit wachsender Größe des Amts (und auch seines Zuständigkeitsgebiets) vom kleinsten (LRA-2) mit einem Verhältnis von 1:1 über das mittlere (LRA-1) mit einem Verhältnis von 1:0,7 bis zum größten (GAAN) mit einem Verhältnis von 1:0,4 in Richtung eines abnehmenden Anteils an Neugenehmigungen.

Zusammengefaßt ergibt die Analyse der möglicherweise genehmigungsrelevanten betrieblichen Ausgangsbedingungen, daß es die Unternehmensgröße ist, bei der sich die Verfahren beim niedersächsischen GAA-N von denen beim baden-württembergischen LRA-1 am deutlichsten abheben. Mit ihr kovariieren jeweils in gewissem - wenn auch nicht in statistisch signifikantem - Maße die Inanspruchnahme bzw. der Verzicht auf externe Beratung und die Art der beantragten Genehmigung. Ein etwaiger Zusammenhang mit der Art der zu genehmigenden Anlage (gem. Anhang der 4 . $\mathrm{BImSchV}$ ) ist dagegen nicht nachzuweisen.

Unternehmensgröße und Anlagenart sind folglich als mögliche intervenierende Variablen in den Vergleich der Verfahrensabläufe unter den beiden Genehmigungsmodellen eingegangen. D.h. die Befunde wurden auf ent-

52. Die Kovariation ist beim GAA-N (das auch jeweils die vergleichsweise höchsten Anteile an Änderungsgenehmigungen und an größeren Unternehmen aufweist) deutlicher ausgeprägt als beim LRA-1, erreicht aber ebenfalls keine Signifikanz (auf Grund der durch Kreuztabellierung reduzierten absoluten Zellhäufigkeiten). Vgl. zu den Werten im einzelnen Tab. 6-A im Anhang. 
sprechende intervenierende Effekte hin überprüft, ohne daß dies (bei negativem Ausgang) jeweils ausdrücklich vermerkt ist. 


\section{Ergebnisse}

Ursprünglich sollten für den Vergleich der unter den beiden Modellen entwickelten Genehmigungspraxis die bei den beiden baden-württembergischen LRÄ erhobenen Daten zu einem gemeinsamen Datenkorpus zusammengefaßt werden. Im Zuge der Aktenauswertung wurden aber beim LRA2 (bei dem aus zugangsbedingten Gründen mit der Datenerhebung begonnen worden war) und bei dem diesem zugeordneten GAA einige eher als atypisch zu betrachtende Randbedingungen sichtbar, bei denen nicht auszuschließen ist, daß sie sich auch im Verwaltungsvollzug niedergeschlagen haben. ${ }^{53}$ Von daher mußte auf eine systematische Berücksichtigung der Befunde zum LRA-2 verzichtet werden. Diese werden zwar nachfolgend ebenfalls ausgewiesen, die Interpretation der Ergebnisse stützt sich aber vorrangig auf den Vergleich zwischen LRA-1 und GAA-N.

\section{Verfahrenseinleitung}

\subsection{Bekanntheit der behördlichen Zuständigkeit bei den Betreibern genehmigungspflichtiger Anlagen und der öffentlichen Verwaltung}

Aus der rechtlichen Konstruktion, die Errichtung und Betrieb bestimmter Anlagen an eine behördliche Genehmigung bindet, folgt die Pflicht des (potentiellen) Betreibers einer solchen Anlage, rechtzeitig, d.h. vor deren Errichtung oder »wesentlicher « Änderung, um eine entsprechende Genehmigung nachzusuchen (auf die er bei Erfüllung der gesetzlichen Vorausset-

53 Auf die Zuständigkeit nur eines einzigen Sachbearbeiters für die Genehmigungsverfahren beim LRA-2 und den damit korrespondierenden vergleichsweise kleinen regionalen Zuständigkeitsbereich dieses Amts wurde bereits hingewiesen (Kap. II, Ziff. 4.1). Hinzu kommt als weitere Besonderheit eine aus gemeinsamen Ausbildungszeiten herrührende persönliche Vertrautheit dieses Sachbearbeiters mit einem Großteil der Kollegen des zugehörigen GAA-2, wobei die räumliche Nähe der beiden Behörden Häufigkeit und Unmittelbarkeit der wechselseitigen Kontakte zusätzlich begünstigt. Das zur Entlastung des bislang zuständigen Amts (erst kurz vor Beginn des Untersuchungszeitraums) neugegründete GAA-2 zeichnet sich zudem (ebenfalls) durch einen vergleichsweise kleineren Zuschnitt im Hinblick auf den regionalen Zuständigkeitsbereich und den Personalbestand aus. Schließlich leitete beim LRA-2 das Amt für Umweltschutz ein Nicht-Jurist, während es wohl eher der (ungeschriebenen) Regel der Stellenbesetzung in der öffentlichen Verwaltung entsprechen dürfte, eine solche Position einem Juristen zu übertragen (wie dies beim LRA-1 auch der Fall war). 
zungen einen Anspruch hat). Ungenehmigtes Errichten, Betreiben oder wesentliches Ändern solcher Anlagen ist grundsätzlich rechtswidrig. ${ }^{54}$

Die baden-württembergische »Verteilung « der Genehmigungszuständigkeit auf die untere Verwaltungsebene der Landkreise und kreisfreien Städte sollte insbesondere eine größere »Bürgernähe« der Verwaltung gewährleisten. ${ }^{55}$ Ein solcher Effekt müßte sich jedoch längerfristig u.a. auch in einer besseren Kenntnis des Bürgers, zumal auch des gewerbetreibenden, über die in seinen Angelegenheiten jeweils zuständige behördliche Stelle niederschlagen.

Nimmt man als Indikator für ein solches Wissen die Häufigkeit von »Irrläufern«, d. h. bei einer nicht-zuständigen Behörde eingegangene Anträge auf Genehmigungserteilung, schneidet das (»bügernähere«) LRA-1 mit einem Anteil von 18,6\% nicht besser, sondern sogar etwas schlechter ab als das (»bürgerfernere«) niedersächsische GAA-N mit einem Anteil von $12,1 \%$. Fast ausnahmslos waren die entsprechenden Genehmigungsgesuche an die Baubehörde adressiert. Offenbar ist in jenen Fällen der Antragsteller davon ausgegangen, es genüge eine (einfache) Baugenehmigung statt der tatsächlich benötigten und voraussetzungsvolleren (zudem auch teuereren) immissionsschutzrechtlichen Genehmigung. ${ }^{56}$ Diese Anteile repräsentieren allerdings nur die entdeckten »Irrläufer«. Offen bleibt daher die Frage nach dem Vorkommen bzw. der Häufigkeit nicht entdeckter Fälle, in denen eine Baugenehmigung erteilt wurde, obwohl das beantragte Vorhaben ein immissionsschutzrechtliches Genehmigungsverfahren erfordert hätte (»Dunkelziffer«). Ein erster Anhaltspunkt für das mögliche Ausmaß läßt sich den unterschiedlichen Wegen entnehmen, die bei den beiden Behörden zur Entdeckung führten. In Niedersachsen leitet die Baubehörde ein entsprechendes Baugenehmigungsverfahren ein und fordert im Rahmen der Fachbehördenbeteiligung das GAA-N zur Stellungnahme auf, das dann an Hand der Antragsunterlagen ggf. das Erfordernis einer immissionsschutzrechtlichen Genehmigung feststellt. Hält das verfahrensleitende Bauamt eine Beteiligung des GAA (als Fachbehörde) für nicht geboten, bleiben mögliche »Irrläufer « unentdeckt. Demgegenüber legt das Bauamt des Landkreises Anträge, bei denen es eine potentielle immissionsschutzrecht-

54 Die Rechtswidrigkeit erfüllt mindestens den Tatbestand einer Ordnungswidrigkeit i. S. d. OWiG, vielfach auch zusätzlich einen Straftatbestand i. S. d. StGB. In diesen Fällen »soll« die zuständige (Genehmigungs-) Behörde nach § $20 \mathrm{Abs}$. 2 BImSchG Stillegung oder Beseitigung anordnen, sie »hat « es tun, »wenn die Allgemeinheit oder die Nachbarschaft nicht auf andere Weise ausreichend geschützt werden kann.«

55 Vgl. oben Anm. 27.

56 Für die Unkenntnis über die Genehmigungsbedürftigkeit solcher Vorhaben spricht auch, daß diese Anträge bei allen drei Genehmigungsbehörden überproportional häufig Neu- und nicht Änderungsgenehmigungen betreffen. Ein Zusammenhang mit der Unternehmensgröße, etwa in dem Sinne, daß kleinere Firmen weniger mit dem Umweltschutzrecht vertraut sind als größere, ist dagegen nicht nachzuweisen. 
liche Genehmigungsbedürftigkeit sieht, vor Einleitung eines Baugenehmigungsverfahrens zur entsprechenden Prüfung der (im selben Haus angesiedelten) Immissionsschutzbehörde vor, das diese Frage dann (wie bei den direkt bei ihr eingegangenen Anträgen in der Regel auch) in Rücksprache mit dem GAA klärt. ${ }^{57}$ An diesen Unterschied der Entdeckungswege läßt sich die Vermutung anknüpfen, daß die baden-württembergische Integration der immissionsschutzrechtlichen Genehmigungsbehörde in die allgemeine Verwaltung einer Blickverengung der einzelnen Fachbehörden auf die je eigenen Belange gegenzusteuern geeignet ist, während die niedersächsische Auslagerung fachbehördlichen Sichtbegrenzungen (im Hinblick auf den Immissionsschutz) eher Vorschub zu leisten scheint. Als »bürgernäher« könnte man die baden-württembergische Verwaltungsorganisation insofern betrachten, als eine frühzeitige Prüfung der im konkreten Fall gegebenen fachlich-rechtlichen Zuständigkeit die Anzahl innerbehördlicher $»$ Durchlaufstationen « von Anträgen auf das erforderliche $\mathrm{Ma}$ begrenzt und insoweit die Verfahrensdauer bis zur Genehmigungserteilung verkürzt.

Das baden-württembergische Organisationsmodell könnte im übrigen auch eine größere »Bürgernähe «, freilich in einem anderen als dem offiziell genannten Sinne, ungewollt begünstigen. Beim LRA-1 wie auch beim LRA-2 fand jeweils ein Fünftel der Verfahren (20,9\% bzw. 20,8\%) unter Beteiligung des zuständigen Dezernenten ${ }^{58}$ - und zugleich Ersten Landesbeamten und in dieser Funktion zusätzlich auch Stellvertreter des Landrats - statt (vgl. oben Kap. II, Ziff. 4.1, Tab. 2.3). Seine Mitwirkung diente ausnahmslos dazu, das beantragte Vorhaben überhaupt bzw. gemäß der betrieblichen Planung zu ermöglichen (was auch erreicht wurde).$^{59}$ In der Mehrzahl der Fälle hatte der Antragsteller um entsprechende Unterstützung beim Dezernenten (im Einzelfall auch beim Landrat als Behördenleiter) nachgesucht unter Hinweis auf eine die (mittelständische) Wirtschaft schädigende »Genehmigungsverhinderungspraxis « der beteiligten Behörden, die der vom Landkreis proklamierten Wirtschaftsförderung zuwiderlaufe. Für die niedersächsischen Genehmigungsverfahren ist ein entsprechendes kommunalpolitisch motiviertes »Engagement« höherer Stellen (bezogen auf die Entscheidungen der beteiligten Behörden) in den Akten nicht dokumentiert. Das schließt natürlich nicht aus, daß ein solches Verhalten im Einzelfall gleichwohl praktiziert wird. Möglich ist aber auch, daß im niedersächsischen Modell der Antragsteller auf einen solchen, für alle Beteiligten (zeit-) aufwendigen Weg zur Durchsetzung seiner Genehmigungsinteressen

57 In Zahlen: Während das LRA-1 die Mehrheit der »Irrläufer « vor Verfahrensbeginn von der unzuständigen Behörde erhielt, war das beim GAA-N lediglich einmal der Fall.

58 Dessen Dezernat umfaßt in beiden LRÄ neben dem Amt für Umweltschutz auch das Baurechtsamt und die Kommunalaufsicht.

$59 \mathrm{Vgl}$. hierzu auch unten Ziff. 3.1.2. 
nicht angewiesen ist. Darauf wird weiter unten zurückzukommen sein (Ziff. 3.1.4).

\subsection{Verfahrensauslösender Anlaß}

Wie erwähnt, liegt nach dem Gesetz die Initiative für die Einleitung eines Genehmigungsverfahrens für Errichtung bzw. Änderung einer genehmigungspflichtigen Anlage formell beim (potentiellen) Betreiber. Diese Verteilung der »Antragslast « ist für die Genehmigungsbehörde solange problemlos, wie der Betreiber ein Eigeninteresse an der Genehmigungserteilung hat und entsprechend auch von sich aus aktiv wird. Dies trifft in der Regel auf die Fälle zu, in denen dem Betreiber die Genehmigungsbedürftigkeit der Anlagenerrichtung bzw. -änderung bekannt ist und er mit deren (rechtswidriger) Durchführung auch noch nicht begonnen hat. Tatsächlich wurde aber nicht einmal die Hälfte aller Genehmigungsverfahren (ohne erkennbaren Anstoß seitens der Behörden) vom Betreiber initiiert, nämlich $48,3 \%$ beim GAA-N und - noch etwas weniger - 41,9\% beim LRA-1, wie Tab. 7 (Kategorie a) zeigt. ${ }^{60}$

Der größere Teil der Verfahren wurde mithin beim GAA-N wie beim LRA-1 auf Anstoß Dritter auf den Weg gebracht, bei beiden Ämtern vorrangig von behördlicher Seite (Kategorien b plus c, vgl. dazu auch weiter unten). Die restlichen Verfahren kamen durch Nachbarbeschwerden (vereinzelt auch durch Presse- und Rundfunkinformationen vermittelt $)^{61}$ in Gang (Kategorie d), wobei im Süden Nachbarbeschwerden etwas stärker hervortreten als im Norden. Dieser Unterschied scheint eher auf eine unterschiedliche »öffentliche Beschwerdekultur « - deren Ausprägung wiederum mit der Besiedlungsdichte zusammenhängen könnte - in den beiden Ländern $^{62}$ hinzudeuten als unterschiedlichem amtlichen Agieren oder der räumlichen »Bürgernähe« der Genehmigungsbehörde zuzurechnen zu sein. ${ }^{63}$ Dafür spricht, daß auch in laufenden Verfahren beim LRA-1 Nachbarn mit sechs Fällen $(=14,0 \%)$ öfter gegen anstehende Genehmigungen

60 Abweichend hier die Verteilung beim LRA-2, was auf den dortigen vergleichsweise hohen Anteil an Neugenehmigungen zurückzuführen sein dürfte (vgl. oben Kap. II, Ziff. 4.2.4, Tab. 6).

61 Bei allen drei untersuchten Ämtern jeweils ein Fall.

62 Vgl. auch die bei LRA-1 und LRA-2 ähnlichen Werte von 14,0\% bzw. 12,5\%.

63 Zusätzlich könnte auch die eigens beim baden-württembergischen Umweltministerium eingerichtete Beschwerdestelle (»Bürgertelefon«) die nachbarliche Gegenwehr ermuntert haben. 
Einwendungen vorbrachten als beim GAA-N mit nur einem Fall $(=1,7 \%){ }^{64}$ Hinzu kommt, daß diese Verfahren - von jeweils einer Ausnahme beim LRA-1 bzw. LRA-2 abgesehen - nicht identisch sind mit jenen, die durch Nachbarbeschwerden in Gang gekommen waren. Diesen war also in der Regel erfolgreich abgeholfen worden. Rechnet man die beiden (zeitversetzten) Formen der Nachbarbeschwerden zusammen (abzüglich der beiden jeweils als Doppelfall zählenden Ausnahmen), so ergibt sich folgendes Bild: Beim LRA-1 haben in $11(=25,6 \%)$, also in einem Viertel der Verfahren Nachbarn Beschwerde geführt, beim GAA-N nur in vier $(=6,9 \%)$ Verfahren. ${ }^{65}$ Der Unterschied ist statistisch signifikant. ${ }^{66}$

Tab. 7

Auslöser der Verfahren

\begin{tabular}{|l|c|c|c|}
\cline { 2 - 4 } \multicolumn{1}{c|}{} & GAA-N & LRA-1 & LRA-2 \\
\hline a) Betreiberinitiative & 28 & 18 & 15 \\
& $48,3 \%$ & $41,9 \%$ & $62,5 \%$ \\
\hline b) Genehmigungsbehörde* & 14 & 9 & 2 \\
& $24,1 \%$ & $20,9 \%$ & $8,3 \%$ \\
\hline c) Sonstige Behörden & 13 & 10 & 4 \\
& $22,4 \%$ & $23,3 \%$ & $16,7 \%$ \\
\hline d) Nachbarn / Medien** & 3 & 6 & 3 \\
\hline \hline Verfahren gesamt*** & $5,2 \%$ & $14,0 \%$ & $12,5 \%$ \\
\hline
\end{tabular}

* Aus Vergleichsgründen im Süden LRA und zugehöriges GAA.

** Presse, Funk.

*** Abweichungen von $100,0 \%$ durch Rundungen bei den prozentualen Einzelhäufigkeiten.

Die von Amtsseite ausgelösten Verfahren (Kategorien b plus c) gehen beim GAA-N wie beim LRA-1 jeweils in etwa zur Hälfte der Fälle auf ein entsprechendes Initiativwerden der Genehmigungsbehörde selbst zurück (Kategorie b), beim LRA-1 regelmäßig - abgesehen von Ausnahmen wie etwa

64 Beim LRA-2 sind es 8,3\% aller Verfahren. - In zwei Fällen (je einer beim LRA-1 bzw. LRA-2) legten die Einwender gegen die Genehmigungserteilung Widerspruch ein, einer davon (LRA-1) mündete in Klageerhebung beim Verwaltungsgericht und war bei Abschluß der Erhebung noch nicht entschieden. Im übrigen ist ein spürbarer verfahrensverlängernder Effekt der Einwendungen nicht auszumachen.

65 LRA-2 nimmt mit vier (=16,7\%) Verfahren eine Mittelposition ein.

$66 \mathrm{chi}^{2}=6,78>6,64, \mathrm{p}=0,01, \mathrm{df}=1$. 
der Durchführung eines ministeriellen Erlasses zur Altanlagensanierung nach Hinweisen des ihm hier aus Vergleichsgründen zugeordneten (»seines «) GAA. Die etwa gleichen Anteile der beiden Genehmigungsbehörden an entdeckten Fällen eines immissionsschutzrechtlich vorschriftswidrigen Anlagenbetriebs (Sanierungsbedarf auf Grund verschärfter Emissionsbzw. Immissionsgrenzwerte, ungenehmigt durchgeführte wesentliche Anlagenänderungen u.ä.) deuten darauf hin, daß das formell nur als Fachbehörde fungierende GAA im Süden die rechtliche Frage des Genehmigungserfordernisses nicht weniger im Blick hat und an die von ihm faktisch getroffene Entscheidung keine grundsätzlich anderen (strengeren oder nachgiebigeren) Maßstäbe anlegt als das zusätzlich mit der Genehmigungszuständigkeit ausgestattete GAA im Norden. In diesem Befund kündigt sich eine (sachliche) Aufgabenverteilung zwischen baden-württembergischem LRA und GAA an, die sich in weiteren Untersuchungsergebnissen bestätigen wird: Entgegen dem offiziellen Organisationsmodell hat sich die faktische Wahrnehmung der Genehmigungskompetenz vom LRA hin zum GAA verlagert (bzw. ist bei letzterem geblieben).

Bei der anderen Hälfte der amtsseitig in Gang gebrachten Verfahren war es in erster Linie die zuständige Baubehörde, die dafür sorgte. ${ }^{67}$ Der $\gg$ Entdeckungsprozeß $\ll$ folgt allerdings in den beiden Modellen von Grund auf anscheinend unterschiedlichen Regeln. Wie in einem anderen Zusammenhang schon angesprochen (Ziff. 1.1), informiert die Baubehörde im Süden das LRA gewissermaßen im Vorgriff über das aus ihrer Sicht mutmaßliche immissionsschutzrechtliche Genehmigungserfordernis für ein bei ihr zur Baugenehmigung eingereichtes Vorhaben, während im Norden die immissionsschutzrechtliche Genehmigungsbedürftigkeit eines beantragten Bauvorhabens gewissermaßen erst im nachhinein von der Genehmigungsbehörde anläßlich der von ihr angeforderten fachbehördlichen Stellungnahme festgestellt wird. Offenbar führen beide Wege zu vergleichbaren Anteilen entdeckter immissionsschutzrechtlich genehmigungsbedürftiger Bauvorhaben. Daraus läßt sich allerdings nicht schließen, daß dies auch für mögliche Anteile an in dieser Hinsicht unentdeckt gebliebenen Bauvorhaben gilt.

Eine auf erteilten Genehmigungen beruhende Untersuchung kann zwangsläufig keine Aussage über das Vorkommen ungenehmigten Anlagenbetriebs treffen, eine Möglichkeit, die durch das Schaffen einer Genehmigungspflicht nicht verhindert, sondern erst erzeugt wird. Eine gewisse Vorstellung davon, in welchem Ausmaß ein zumindest zeitweiliges (bis zur Entdeckung durch die Behörden) ungenehmigtes Betreiben von einschlägigen Anlagen überhaupt stattfindet, kann der Befund geben, daß in rd. 30\%

67 Im übrigen waren es insbesondere für andere umweltschutzrechtliche Belange zuständige Ämter wie die Wasser- und die Abfallbehörde. 
der Verfahren (GAA-N: $18=31,0 \%$, LRA-1: $13=30,2 \%)^{68}$ die zur Genehmigung anstehende Anlagenänderung oder Anlagenerrichtung bereits vor Antragstellung durchgeführt war. Aufgedeckt wurden die ungenehmigten Zustände, die sogar vergleichsweise häufiger Neu- als Änderungsgenehmigungen betrafen, ${ }^{69}$ in erster Linie bei Gelegenheit anders veranlaßter Kontrollen des GAA und (etwas weniger) anderer Fachbehörden, zum geringeren Teil durch Nachbarbeschwerden und (in seltenen Fällen) auch durch unaufgeforderte (»nachträgliche«) Antragstellung des Betreibers selbst.

\subsection{Behördliche Vorantragsberatung}

\subsubsection{Häufigkeit von Vorantragsberatungen}

Eine frühzeitige Beratung des potentiellen Antragstellers durch die Genehmigungsbehörde hinsichtlich der Erfordernisse des Genehmigungsantrags ist ausdrücklich gesetzlich vorgesehen. ${ }^{70}$ Sie erfolgte regelmäßig in allen von Amtsseite bzw. durch Nachbarbeschwerden ausgelösten Verfahren. ${ }^{71}$ Aber auch bei der Mehrzahl der vom Betreiber selbst initiierten Verfahren ging eine Beratung durch die Genehmigungsbehörde (im Süden einschließlich des zugehörigen GAA) voraus, wobei der Anteil der ohne (dokumentierte) Beratung eingereichten Anträge beim GAA-N höher ausfällt als

68 LRA-2: $6=25,0 \%$.

69 Bezogen auf die jeweiligen Anteile an Neu- bzw. Änderungsgenehmigungsverfahren (vgl. oben Kap. II, Ziff. 4.2.4, Tab. 6) belaufen sich die einschlägigen (wohlgemerkt: entdeckten bzw. aktenkundigen) Fälle ungenehmigter Anlagenerrichtung bzw. -änderung beim LRA-1 auf sieben $(=41,2 \%)$ bei den Neugenehmigungen und auf sechs $(23,1 \%)$ bei den Änderungsgenehmigungen, beim GAA-N auf fünf (= $31,3 \%)$ bei den Neugenehmigungen und $11(=26,2 \%)$ bei den Änderungsgenehmigungen. Diese, vielleicht eher überraschenden Relationen sind vor dem Hintergrund zu sehen, daß ein nicht unerheblicher Teil der Änderungsgenehmigungen auf einen behördlich festgestellten immissionsschutzrechtlichen Sanierungsbedarf der Anlage zurückging. Zwar ist das Betreiben einer genehmigungsrechtlich sanierungsbedürftigen Anlage ebenfalls genehmigungswidrig, die Genehmigungswidrigkeit resultiert dort allerdings aus einer (passiv) unterlassenen und nicht aus einer »eigenmächtig « (aktiv) ungenehmigt vorgenommenen Anlagenänderung, um die es im vorliegenden Zusammenhang geht.

$70 \S 2$ Abs. 2 Satz 1 der 9. BImSchV verpflichtet die Genehmigungsbehörde ausdrücklich zur Beratung, sobald sie vom Vorhabensträger über dessen Planung unterrichtet wird. Die Beratungspflicht erstreckt sich nicht nur auf die (förmliche) Anstellung, sondern auch auf den zeitlichen Ablauf des Genehmigungsverfahrens sowie die Erörterung sonstiger »für die Durchführung des Verfahrens erhebliche(r) Fragen«.

71 Ausgenommen je ein durch eine sonstige Fachbehörde in Gang gebrachtes Verfahren beim GAA-N bzw. LRA-1. 
beim LRA-1 ${ }^{72}$ Im Falle der Betreiberinitiative ist die behördliche Vorantragsberatung allerdings von einer entsprechenden Nachfrage des Antragstellers abhängig, die sich weitgehend dem Einfluß der Genehmigungsbehörde entzieht.

Insgesamt haben beim GAA-N in $82,8 \%$ und beim LRA-1 in $90,7 \%$ aller Genehmigungsverfahren Vorantragsberatungen stattgefunden, entsprechend sind $17,2 \%$ bzw. 9,3\% ohne vorherige Beratung eingeleitet worden. ${ }^{73}$ Die Verteilung der Verfahren nach der Anzahl der im einzelnen durchgeführten Beratungen zeigt Tab. $8 .^{74}$

Bei beiden Behörden, auf die sich der vorliegende Vergleich der zwei Genehmigungsmodelle im wesentlichen konzentriert, wurden jeweils in etwa zwei Dritteln der Verfahren zwei oder mehr Besprechungstermine abgehalten. ${ }^{75}$ Dabei zeigt sich, daß das LRA-1 höhere Anteile als das GAA$\mathrm{N}$ an besonders beratungsintensiven Verfahren mit fünf oder sechs Terminen sowie mit mehr als sieben Terminen aufweist. Auch das Maximum an Beratungsterminen pro Fall liegt beim LRA-1 mit 20 Terminen deutlich über dem beim GAA-N mit 12 Terminen. ${ }^{76}$ Die Tendenz zu einer höheren Kontaktdichte mit dem Antragsteller beim LRA-1 gegenüber dem GAA-N setzt sich auch in der offiziellen Verfahrenslaufzeit fort (vgl. unten Ziff. 2.4.1).

72 GAA-N: $9(32,1 \%)$ von 28 Verfahren, LRA-1: $3(16,7 \%)$ von 18 Verfahren (LRA2: $5(33,1 \%)$ von 15 Verfahren); der Unterschied zwischen GAA-N und LRA-1 ist allerdings statistisch nicht signifikant $\left(\right.$ chi $^{2}=0,87$ (Yates' Korrektur) $<2,71$ für $p=$ 0,1 und $\mathrm{df}=1$ ).

73 Die Abweichungen der Anteile zwischen den drei Genehmigungsbehörden sind statistisch nicht signifikant (GAA-N zu LRA-1: chi $^{2}=1,36<2,71$; LRA-1 zu LRA-2: chi $^{2}=1,32$ (Yates' Korrektur) $<2,71$ jeweils für $p=0,1$ und $\mathrm{df}=1$ ).

74 Die Klassengrenzen wurden auf der Grundlage der Rohverteilungen gezogen.

75 GAA-N: $67,2 \%$, LRA-1: $65,1 \%$ (LRA-2: 58,3\%).

76 Die Verschiebung zugunsten höherer Beratungshäufigkeit beim LRA-1 gegenüber GAA-N zeigt auch der Vergleich einzelner Verteilungsparameter: Das arithmetische Mittel beträgt beim GAA-N 3,0 (2,95), beim LRA-1 dagegen 4,5 (4,49) Beratungstermine pro Verfahren, wobei die Einzelwerte beim LRA-1 mit einer Varianz von 20,0 (19,92) statistisch signifikant stärker um den Mittelwert streuen als beim GAA$\mathrm{N}$ mit einer Varianz von $7,0(6,96)\left[\mathrm{F}=2,86>\mathrm{F}_{(0,999 ; 42,57)} 2,68\right]$, so daß schon von daher die Mittelwerte nicht vergleichbar sind. - Die Werte beim LRA-2 mit einem arithmetischen Mittel von 2,7 (2,71) und einer Varianz von 9,0 (8,56) sind dagegen denen des GAA-N (statistisch) vergleichbar $\left(F=1,23<F_{(0,975 ; 57,23)} 2,08 ; t=0,363\right.$ $\left.<\mathrm{t}_{(0,975 ; 80)} 1,99\right)$. 
Tab. 8

Anzahl an Vorantragsberatungen durch die Genehmigungsbehörde* (Häufigkeitsverteilung)

\begin{tabular}{|l|c|c|c|}
\cline { 2 - 4 } \multicolumn{1}{c|}{} & $G A A-N$ & $L R A-I$ & $L R A-2$ \\
\hline Keine & 10 & 44 & 5 \\
& $17,2 \%$ & $9,3 \%$ & $20,8 \%$ \\
\hline Eine & 9 & 11 & 5 \\
& $15,5 \%$ & $25,6 \%$ & $20,8 \%$ \\
\hline Zwei & 12 & 5 & 6 \\
Drei bis vier & $20,7 \%$ & $11,6 \%$ & $25,0 \%$ \\
\hline Fünf bis sechs & 14 & 5 & 4 \\
\hline Sieben und mehr** & $24,1 \%$ & $11,6 \%$ & $16,7 \%$ \\
\hline \hline Verfahren gesamt*** & 9 & 9 & 1 \\
\hline
\end{tabular}

* Aus Vergleichsgründen im Süden LRA und zugehöriges GAA.

** Maximale Zahl an Beratungsterminen pro Fall beim LRA-1: 20, beim GAA-N: 12, beim LRA-2: 10 Termine.

*** Abweichungen von 100,0\% durch Rundungen bei den prozentualen Einzelhäufigkeiten.

\subsubsection{Mitwirkung anderer Behörden}

Ergab die rein quantitative Betrachtung der Beratung bereits einen insgesamt größeren Umfang beim LRA-1 im Vergleich zum GAA-N, so treten bei qualitativer Betrachtung stärker ausgeprägte Differenzen zutage (Tab. 9). Als ein Indikator für die Qualität (zu einem weiteren Indikator s. nachfolgende Ziff. 1.3.3) dient hier die - rechtlich ausdrücklich zulässige - 77 Beiziehung mutmaßlich in ihren Belangen betroffener Fachbehörden (im Süden unter Ausnahme des zugehörigen GAA) durch die Genehmigungsbehörde. $^{78}$

$77 \S 2$ Abs. 2 Satz 2 der 9. BImSchV.

78 Diese Operationalisierung von Beratungsqualität mißt also die Unterstützung der Beratung durch sonstigen genehmigungsrelevanten fachspezifischen Sachverstand. Schlußfolgerungen über die fachliche und/oder rechtliche Güte der einzelnen Beratung (deren Messung wegen der damit verbundenen methodischen Probleme im Hinblick auf die Objektivität hier unterblieben ist) lassen sich daraus nicht ziehen. 
Tab. 9

Mitwirkung anderer Behörden bei der Vorantragsberatung

\begin{tabular}{|l|c|c|c|}
\cline { 2 - 4 } \multicolumn{1}{c|}{} & GAA-N & LRA-1 & LRA-2 \\
\hline Nur Genehmigungsbehörde* & 37 & 26 & 16 \\
& $77,1 \%$ & $66,7 \%$ & $84,2 \%$ \\
\hline Genehmigungsbehörde mit sonstigen Be- & 11 & 13 & 3 \\
hörden & $22,9 \%$ & $33,3 \%$ & $15,8 \%$ \\
[darunter: Vorantragskonferenzen] & {$[5]$} & {$[8]$} & {$[1]$} \\
& {$[10,4 \%]$} & {$[20,5 \%]$} & {$[5,3 \%]$} \\
\hline Verfahren mit Beratung & 48 & 39 & 19 \\
& $100,0 \%$ & $100,0 \%$ & $100,0 \%$ \\
\hline \multirow{2}{*}{ Verfahren ohne Beratung } & 10 & 4 & 5 \\
& $17,2 \%$ & $9,3 \%$ & $20,8 \%$ \\
\hline \hline \multirow{2}{*}{ Verfahren gesamt } & 58 & 43 & 24 \\
\hline
\end{tabular}

* Aus Vergleichsgründen im Süden LRA und zugehöriges GAA.

Zwar fand in der großen Mehrzahl der Fälle im Norden wie im Süden die Beratung ausschließlich durch die Genehmigungsbehörde (im Süden aus Vergleichsgründen unter Einschluß des zugehörigen GAA) statt. Das LRA1 zog aber öfter zusätzlich mindestens eine sonstige, voraussichtlich in ihren Belangen betroffene Fachbehörde hinzu als das GAA-N (33,3\% gegenüber $22,9 \%$, bezogen auf Verfahren mit Beratung $)^{79}$. Darüber hinaus waren beim LRA-1 diese Beratungen in 8 Fällen (20,5\% aller Verfahren mit Beratung) in Form der gemeinsamen Beteiligung mehrerer sonstiger Fachbehörden als sog. Vorantragskonferenzen ausgestaltet, ${ }^{80}$ beim GAA-N dagegen lediglich in 5 Fällen (10,4\% aller Verfahren mit Beratung) ${ }^{81}$ Auf Grund der geringen absoluten Fallzahlen bei beiden Behörden bleibt die Differenz

79 Die Differenz liegt allerdings unterhalb der Signifikanzgrenze $\left(\mathrm{chi}^{2}=1,13<2,71\right.$ für $\mathrm{p}=0,1$ und $\mathrm{df}=1$ ). Ebenfalls statistisch nicht signifikant ist die diesbezügliche Anteilsdifferenz zwischen LRA-1 und LRA-2 $\left(\mathrm{chi}^{2}=1,90<2,71\right.$ für $\mathrm{p}=0,1$ und df $=1$ ).

80 Der Begriff Vorantrags- bzw. Antragskonferenz ist eine Wortschöpfung ministerieller Erlasse zur Verfahrensbeschleunigung im Hinblick auf die Regelung in $\S 2$ Abs. 2 Satz 2 der 9. BImSchV. Er wird hier großzügig gehandhabt und schließt alle Fälle ein, in denen bei einem Beratungstermin mindestens zwei sonstige Fachbehörden (im Süden ausgenommen das zuständige GAA) anwesend sind.

81 LRA-2 weist nur einen einzigen Fall (5,3\% der 19 Verfahren mit Beratung) mit Vorantragskonferenz auf. 
aber statistisch unerheblich. ${ }^{82}$ Im übrigen reduziert sie sich deutlich, wenn man den weiteren Verfahrensverlauf hinzunimmt. Denn nach offiziellem Verfahrensbeginn fanden beim GAA-N mehr Antragskonferenzen statt als beim LRA-1, wobei allerdings auch hier die absoluten Fallzahlen niedrig ausfallen (vgl. hierzu näher unten Ziff. 2.4.2).

Im Süden führte die Genehmigungsbehörde den Großteil ihrer Beratung in Kooperation mit dem zugehörigen GAA durch. Es verbleibt eine Minderzahl von 8 Fällen (20,5\% aller Verfahren mit Beratung), in denen das LRA-1 die Beratung allein vornahm. ${ }^{83}$ In der Hälfte der Fälle hatte sich der Betreiber von sich aus an die Genehmigungsbehörde gewandt, in der anderen Hälfte hatte diese den Betreiber auf die durch gesetzliche Änderungen entstandene Genehmigungsbedürftigkeit seiner Anlage hingewiesen und ihn zur Antragstellung aufgefordert.

Kennzeichnend für die Beratungstätigkeit des LRA bleibt gleichwohl, $\mathrm{da}$ es mangels zureichenden eigenen (naturwissenschaftlich-technischen) Fachwissens den Sachverstand des GAA in Fragen der Antragsausarbeitung in Anspruch nehmen muß, ggf. auch nur, um sich gegenüber dem Antragsteller abzusichern. Der Umstand, daß beim LRA das Personal in diesem Wissensbereich nicht professionell geschult ist, könnte auch erklären, daß es zudem häufiger andere, ebenfalls hauptsächlich auf naturwissenschaftlich-technische Belange spezialisierte Fachbehörden bei der Beratung hinzuzieht als das GAA-N, dessen Mitarbeiter sich (nicht nur innerhalb ihres eigenen fachlichen Zuständigkeitsbereichs) auf Grund ihrer technischen Basisqualifikation ein eigenes Urteil zutrauen.

\subsubsection{Medien der Kommunikation bei der Vorantragsberatung}

Ein weiterer Indikator für die (qualitative) Intensität der Beratung ist das Medium der Kontakte. Dabei lassen sich zunächst zwei Grundformen der Kommunikation unterscheiden, eine medial vermittelte in der Form der schriftlichen oder telefonischen Kommunikation und eine unter physischer Anwesenheit. Im zweiten Fall wird zusätzlich nach dem Ort differenziert, d.h. danach, ob die Beratung in der Behörde oder in der Firma des Antragstellers erfolgte.

Wie Tab. 10 zeigt, machen Beratungskontakte, die sich auf Schriftwechsel (einschl. Fax) oder Telefonate beschränken (Kategorie a), beim GAAN wie beim LRA-1 jeweils rd. ein Fünftel (20,8\% bzw. 20,5\%) der einschlä-

82 Für die Anteile der Fälle mit Vorantragsberatung ergibt sich, bezogen auf die jeweilige Gesamtzahl der Beratungsfälle, ein Testwert von chi $^{2}=1,77<2,71$ und, bezogen auf die jeweilige Gesamtzahl der Verfahren, ein Testwert von $\mathrm{chi}^{2}=2,26<2,71$, jeweils für $\mathrm{p}=0,1$ und $\mathrm{df}=1$.

83 Beim LRA-2 sind es 7 Fälle (36,8\% aller Verfahren mit Beratung). 
gigen Verfahren mit Vorantragsberatung aus. ${ }^{84}$ In den übrigen Fällen fand mindestens ein Beratungsgespräch auch unter persönlicher Anwesenheit (beim LRA-1 stets unter Beteiligung des zugehörigen GAA) statt.

Tab. 10

Medium der Kommunikation bei der behördlichen Vorantragsberatung*

\begin{tabular}{|l|c|c|c|}
\cline { 2 - 4 } \multicolumn{1}{c|}{} & $G A A-N$ & $L R A-1$ & $L R A-2$ \\
\hline a) Nur schriftl./telefonisch & 10 & 8 & 9 \\
& $20,8 \%$ & $20,5 \%$ & $47,4 \%$ \\
\hline \multirow{2}{*}{ b) Auch persönlich, aber nur im Amt } & 3 & 5 & 6 \\
& $6,3 \%$ & $12,8 \%$ & $31,6 \%$ \\
\hline & 35 & 26 & 4 \\
c) Auch persönlich in der Firma & $72,9 \%$ & $66,7 \%$ & $21,1 \%$ \\
[c1) darunter: zusätzl. auch im Amt] & {$[4]$} & {$[2]$} & {$[0]$} \\
& {$[8,3 \%]$} & {$[5,1 \%]$} & {$[-]$} \\
\hline Verfahren mit Beratung** & 48 & 39 & 19 \\
\hline \multirow{2}{*}{ Verfahren ohne Beratung } & $100,0 \%$ & $100,0 \%$ & $100,0 \%$ \\
\hline \hline Verfahren gesamt & 10 & 4 & 5 \\
\hline
\end{tabular}

* Aus Vergleichsgründen im Süden LRA und zugehöriges GAA.

** Abweichungen von $100,0 \%$ durch Rundungen bei den prozentualen Einzelhäufigkeiten.

Vorwiegender Ort der Begegnung war dabei die Firma des Antragstellers (Kategorie c) mit einem Beratungsanteil von $72,9 \%$ beim GAA-N und 66,7\% beim LRA-1. Im Vergleich dazu spielen die Fälle, in denen der Antragsteller zum persönlichen Gespräch im Amt, sei es in der Genehmigungsbehörde, sei es im GAA oder auch in einer anderen Fachbehörde, ${ }^{85}$ erschien oder dorthin gebeten worden war, mit einem Anteil von 14,6\% bzw. 17,9\% (Kategorien b plus c1 im Kleindruck) in beiden Modellen nur

84 Beim LRA-2 sind es mit 47,4\% fast die Hälfte der Beratungsfälle. Das geht entsprechend zu Lasten der Anteile von Kontakten im Amt oder im Betrieb. Von daher ist die Beratungssituation dort mit der bei den beiden anderen Genehmigungsbehörden nicht vergleichbar, so daß auf das LRA-2 bei der Darstellung der weiteren Aufschlüsselung der Befunde nicht näher eingegangen wird.

85 Hierunter befindet sich auch der Hauptteil der als Vorantragskonferenzen ausgestalteten Beratungen. Die restlichen Vorantragskonferenzen finden sich unter den Fällen, in denen auch eine Beratung vor Ort stattgefunden hat. 
eine untergeordnete Rolle. Der mit der Dezentralisierung der Genehmigungszuständigkeit in Baden-Württemberg angestrebte Effekt eines erleichterten (persönlichen) Zugangs zu den Ämtern (»Bürgernähe«) ist insoweit nicht nachzuweisen. ${ }^{86}$

Tab. 10.1

Ausgestaltung der behördlichen Vorantragsberatung ${ }^{*}$ in der Firma des Antragstellers (Anteile bezogen auf die Beratungsverfahren)

\begin{tabular}{|l|c|c|c|}
\cline { 2 - 4 } \multicolumn{1}{c|}{} & GAA-N & LRA-1 & LRA-2** \\
\hline a) Nur Beratungsgespräch & 18 & 13 & 1 \\
& $51,4 \%$ & $50,0 \%$ & \\
\hline \multirow{2}{*}{ b) Nur informatorische Besichtigung } & 3 & 2 & 0 \\
\hline c) Gezielte Betriebskontrolle & $8,6 \%$ & $7,7 \%$ & \\
\hline \multirow{2}{*}{ d) Turnusgemäße Betriebsrevision } & 9 & 11 & 3 \\
& $25,7 \%$ & $42,3 \%$ & 0 \\
\hline \hline Beratungsfälle in der Firma gesamt & 5 & 0 & 0 \\
\hline \hline Beratungsverfahren gesamt & $14,3 \%$ & $0,0 \%$ & 4 \\
\hline
\end{tabular}

* Aus Vergleichsgründen im Süden LRA und zugehöriges GAA.

** Keine Prozentuierung wegen zu kleiner Gesamtfallzahl.

Einen genaueren Einblick in die Ausgestaltung der in der Firma des Antragstellers abgehaltenen Beratungen geben Tab. 10.1 und Tab. 10.2. Dort sind die einschlägigen Verfahren jeweils danach aufgeschlüsselt, wieweit sich der behördliche Besuch auf Gespräche im Firmenbüro beschränkte (Kategorie a) und wieweit er auch Betriebsbegehungen einschloß, wobei jene Fälle nach bloßer informatorischer Besichtigung (Kategorie b), anlaßbezogener Betriebskontrolle (Kategorie c) und routinemäßiger Betriebskontrolle (Kategorie d) unterschieden sind. Den in den beiden Tabellen aus-

86 Das gilt auch für das LRA-2 mit seinem vergleichsweise kleinen regionalen Zuständigkeitsbereich. Der dortige Anteil an Kontakten im Amt in Höhe von 31,6\% unterscheidet sich nicht signifikant von den jeweils entsprechenden Anteilen beim LRA-1 bzw. GAA-N, und zwar unabhängig davon, ob man diese nur auf die Beratungsfälle (Prozentuierungsbasis der in Tab. 10 ausgewiesenen Anteile) oder auf die Gesamtheit der Verfahren bezieht. 
gewiesenen relativen Häufigkeiten liegt als Berechnungsbasis einmal die Teilmenge der mit Beratung einhergegangenen Verfahren zugrunde (Tab. 10.1), das andere Mal dagegen die Gesamtmenge aller Verfahren (Tab. 10.2). Die relativen Anteile, auf die die nachfolgende Beschreibung der Befunde Bezug nimmt, entstammen der zweiten Tabelle.

Tab. 10.2

Ausgestaltung der behördlichen Vorantragsberatung* in der Firma des Antragstellers (Anteile bezogen auf alle Verfahren)

\begin{tabular}{|l|c|c|c|}
\cline { 2 - 4 } \multicolumn{1}{c|}{} & $G A A-N$ & $L R A-1$ & $L R A-2$ \\
\hline a) Nur Beratungsgespräch & 18 & 13 & 1 \\
& $31,0 \%$ & $30,2 \%$ & $5,2 \%$ \\
\hline b) Nur informatorische Besichtigung & 2 & 2 & 0 \\
& $5,2 \%$ & $4,7 \%$ & $0,0 \%$ \\
\hline c) Gezielte Betriebskontrolle & 9 & 11 & 3 \\
\hline d) Turnusgemäßfe Betriebsrevision & $15,5 \%$ & $25,6 \%$ & $0,0 \%$ \\
\hline \hline Beratungsfälle in der Firma gesamt & 5 & 0 & 0 \\
& $8,6 \%$ & $0,0 \%$ & $0,0 \%$ \\
\hline \hline Beratungsverfahren gesamt & 35 & 26 & 4 \\
& $50,3 \%$ & $60,5 \%$ & $16,7 \%$ \\
\hline
\end{tabular}

*Aus Vergleichsgründen im Süden LRA und zugehöriges GAA.

Danach fanden beim GAA-N wie beim LRA-1 mit $31,0 \%$ bzw. $30,2 \%$ jeweils in einem knappen Drittel aller Genehmigungsverfahren Beratungen zwar in der Firma statt, jedoch ohne begleitende Betriebsbegehungen (Kategorie a). Überproportional häufig handelte es sich dabei um größere Unternehmen, die die Behörden zu sich geladen hatten, um diesen dann unter Hinzuziehung ihres leitenden Fachpersonals gegenüberzutreten (»Heimspiel «). In den übrigen Beratungsfällen vor Ort, die insgesamt $27,5 \%$ bzw. 30,3\% der Verfahren beim GAA-N bzw. LRA-1 ausmachen (Kategorien b plus c plus d), war die Initiative jeweils von der Genehmigungsbehörde (bzw. beim LRA-1 zumeist von seiten des GAA) ausgegangen. In erster Linie galten die Termine einer Betriebskontrolle i.S. einer Überwachungsmaßnahme nach $\S 52$ BImSchG (Kategorien c plus d), die dann auf Grund 
des Prüfergebnisses das jeweilige Genehmigungsverfahren auslöste. ${ }^{87}$ Bloße informatorische Besichtigungen (Kategorie b) zur Hebung der fallspezifischen behördlichen Beratungskompetenz bilden dagegen die Ausnahme.

In rd. einem Viertel der Verfahren führten GAA-N $(24,1 \%)$ wie auch LRA-1 (25,3\%) Betriebskontrollen bereits im Vorantragszeitraum durch (Kategorien c plus d). Die Anlässe dafür waren allerdings unterschiedlich. Beim GAA-N handelt es sich in gut zwei Dritteln dieser Fälle um fallspezifisch begründete Kontrollen (Kategorie c) und im knappen restlichen Drittel um Kontrollen im Rahmen turnusgemäßer Betriebsrevisionen (Kategorie d). Demgegenüber resultieren beim LRA-1 sämtliche Kontrollen ausschließlich aus konkreten, fallbezogenen Anlässen. Fälle, die sich generellen Revisionen verdanken, fehlen völlig. Diese unterschiedliche Konstellation dürfte sich weniger aus den unterschiedlichen Genehmigungsmodalitäten erklären als vielmehr aus (situativen) Besonderheiten der Gewerbeaufsichtsämter in Ausübung der ihnen im Norden wie im Süden zukommenden Funktion als zuständige technische Überwachungsbehörden. In Ausübung dieser Funktion ist den Gewerbeaufsichtsämtern in den meisten Bundesländern (durch die zuständigen Ministerien) eine regelmäßige, turnusgemäße Revision der Betriebe aufgegeben. Die Richtwerte für den Überwachungsturnus umfassen dabei i.d.R. relativ große Zeiträume, die nach Betriebsgröße gestaffelt - in etwa zwischen 5 und 10-12 Jahren liegen. Es ist allerdings ein offenes Geheimnis, daß in der Praxis die Richtwerte regelmäßig und vielfach nicht unerheblich überschritten werden, weil das Personal mit der Bewältigung des aktuellen Tagesgeschäfts ausgelastet ist. Entsprechend gering ist die Wahrscheinlichkeit, daß in einem relativ kleinen Zeitraum von drei Jahren, den die vorliegende Untersuchung umfaßt, in nennenswerter Zahl immissionsschutzrechtswidrige Anlagenzustände im Zuge eines regulären Revisionsturnus aufgedeckt werden. Die beim GAA-N festgestellte Häufung so entdeckter Genehmigungsfälle dürfte darauf zurückzuführen sein, daß dort im Untersuchungszeitraum als vorrangiger Arbeitsschwerpunkt den Mitarbeitern die systematische Revision aller von ihnen betreuten »BImSch«-Anlagen aufgegeben war. Eine solche Sondermaßnahme hat es in dieser Zeit bei den beiden süddeutschen GAÄ nicht gegeben. Vielmehr existierte in Baden-Württemberg (aus Gründen der Personaleinsparung) nicht einmal der Form nach ein verbindlicher Überwachungsturnus.

Unabhängig vom Anlaß der Betriebskontrollen bleibt festzuhalten, daß sich im Norden wie im Süden die Genehmigungsbehörde (bzw. und/oder das GAA im Süden) im Rahmen der Vorantragsberatung jeweils in rund zwei Fünfteln dieser Verfahren (bzw. einem Viertel aller Verfahren) über die betriebliche Situation speziell im Hinblick auf den Genehmigungsge-

$87 \mathrm{Vgl}$. hierzu auch oben Ziff. 1.2. 
genstand aus eigener Anschauung und nicht nur durch schriftliche Unterlagen der Firma informiert hatte. Nach offiziellem Verfahrensbeginn, also im Verlauf der Antragsbearbeitung, kamen bei beiden Behörden weitere Besichtigungen bis dahin noch nicht kontrollierter Betriebe hinzu, im Süden mit 23,3\% etwas mehr als im Norden mit 15,5\% (vgl. dazu näher unten Ziff. 2.4.5).

\subsubsection{Gegenstände der Vorantragsberatung}

Naheliegenderweise geht es in Vorantragsberatungen in erster Linie um die immissionsschutzrechtliche Beurteilung der in Frage stehenden Anlage nach dem BImSchG. Das reicht von der Feststellung der Genehmigungspflicht überhaupt (was in allen Fällen entsprechend der Untersuchungsgrundlage erteilter Genehmigungen bejaht wird) und der Zuordnung zu den Ziffern des Anhangs zur 4. BImSchV über die Frage einer Neu- oder Änderungsgenehmigung bis hin zu den Anlagenteilen (auf die sich die Genehmigungspflicht erstreckt) und den für die Antragstellung erforderlichen Unterlagen über die Anlage. Der durch die Amtsautorität »privilegierten « Sicht der Behörde fügten sich die Betreiber mehrheitlich, aber nicht durchgängig. ${ }^{88}$

Über die instruierende Funktion hinaus diente der Vorantragskontakt den Behörden auch dazu, in den Fällen, in denen sie Anhaltspunkte für einen genehmigungswidrigen bzw. sanierungsbedürftigen Anlagenzustand hatten, den Betreiber zur Herstellung eines ordnungsgemäßen Betriebs zu bewegen. Die hauptsächlich eingesetzten Instrumente waren dabei das Einfordern gutachtlicher Prüfbelege und Meßnachweise über Emissionen/Immissionen bzw. zur Sicherheitstechnik der Anlage, die Verpflichtung zur Erarbeitung eines Sanierungskonzepts und zur Einleitung entsprechender Sanierungsmaßnahmen, Fristsetzungen für die Beantragung der erforderlichen (Änderungs-) Genehmigung und schließlich auch die Androhung und ggf. Vollstreckung von Verwaltungszwang, um den behördlichen Forderungen Nachdruck zu verleihen.

Tab. 11 zeigt die Häufigkeiten der jeweils eingesetzten Mittel. Die größten - und auch statistisch signifikanten - Abweichungen zwischen GAA-N und LRA-1 bestehen danach zum einen bei der Einforderung gutachtlicher Prüfbelege bzw. Meßnachweise (Kategorie a), zum anderen bei den Fristsetzungen für Sanierungsmaßnahmen bzw. -konzepte (Kategorie b). Immissionsschutzbezogene Prüfbelege oder Meßnachweise wurden beim LRA-1 - und zwar regelmäßig vom GAA-1 als zuständiger Fachbehörde - mit ei-

88 In der Häufigkeit der genannten Gegenstände gibt es zwischen GAA-N und LRA1 keine auffälligen Unterschiede. Zu inhaltlichen Kontroversen s. näher unten Ziff. 2.4.4 u. 3.1. 
nem Anteil von 46,2\% aller Beratungsfälle mehr als doppelt so häufig wie beim GAA-N mit einem Anteil von nur $22,9 \%$ verlangt. ${ }^{89}$ Während dort allerdings die geforderten Nachweise ausnahmslos im Laufe des Verfahrens auch erbracht wurden, konnte sich beim LRA-1 der Antragsteller in drei Fällen der Erfüllung der fachbehördlichen Forderungen des GAA-1 letztlich mit mehr oder weniger tatkräftiger Unterstützung der Genehmigungsinstanz erfolgreich widersetzen.

Tab. 11

Behördliche Maßnahmen (Auswahl) als Gegenstand der Vorantragsberatung

\begin{tabular}{|l|c|c|c|}
\cline { 2 - 4 } \multicolumn{1}{c|}{} & $G A A-N$ & $L R A-1$ & $L R A-2$ \\
\hline $\begin{array}{l}\text { a) Einforderung gutachterlicher } \\
\text { Prüfbelege/Meßnachweise }\end{array}$ & 11 & 18 & 2 \\
\hline $\begin{array}{l}\text { b) Fristsetzung für Sanierungskonzept/ } \\
\text {-maßnahmen }\end{array}$ & $22,9 \%$ & $46,2 \%$ & $10,5 \%$ \\
\hline c) Fristsetzung für Antragseinreichung & $8,3 \%$ & $23,1 \%$ & $10,5 \%$ \\
\hline d) Androhung von Verwaltungszwang* & 14 & 13 & 7 \\
& $29,1 \%$ & $33,3 \%$ & $36,8 \%$ \\
\hline e) Vollstreckung von Zwangsmaßnahmen & 7 & 6 & 2 \\
& $14,6 \%$ & $15,3 \%$ & $10,5 \%$ \\
\hline f) Von mind. einer Maßnahme betroffene & $4,4 \%$ & 1 & 0 \\
Fälle gesamt & 24 & $2,6 \%$ & $0,0 \%$ \\
\hline \hline Verfahren mit Beratung** & $50,0 \%$ & 27 & 7 \\
\hline Verfahren ohne Beratung & 48 & $69,2 \%$ & $36,8 \%$ \\
\hline \hline Verfahren gesamt & $100,0 \%$ & $100,0 \%$ & $100,1 \%$ \\
\hline \hline
\end{tabular}

* Stillegungsverfügungen, nachträgliche Anordnungen, Betriebsuntersagung, Bußgeld (wegen ungenehmigten Betriebs).

** Da sich die Kategorien der ausgewählten Beratungsgegenstände nicht wechselseitig ausschlieBen (Mehrfachnennungen), kann die Summe der absoluten bzw. relativen Häufigkeiten unter oder über der Gesamtzahl der Beratungsverfahren bzw. unter oder über 100\% liegen.

$89 \mathrm{chi}^{2}=5,23>5,02, \mathrm{p}=0,025, \mathrm{df}=1$. 
Berücksichtigt man - im Vorgriff auf die unten in Ziff. 2.4.4. dargestellten Befunde - weiter, daß beim GAA-N nach offiziellem Verfahrensbeginn in weiteren sechs Fällen, beim LRA-1 jedoch nur in weiteren drei Fällen immissionsschutzbezogene Gutachten auf behördliches Verlangen beigebracht wurden, verringert sich der Vorsprung des Südens gegenüber dem Norden in der Bilanz. Zwar wurden beim LRA-1 am Ende in $41,8 \%$ aller Verfahren einschlägige Gutachten vorgelegt und damit der vergleichbare Anteil in Höhe von $29,3 \%$ beim GAA-N immer noch spürbar übertroffen, doch bleibt die Differenz von 12,5 Prozentpunkten unterhalb der statistischen Signifikanzgrenze. ${ }^{90}$ Der Befund deutet aber eindeutig darauf hin, daß die zusätzliche Genehmigungskompetenz der (niedersächsischen) technischen Fachbehörde nicht als Mittel dient, die fachtechnischen Anforderungen zu erhöhen. Vielmehr verweist er eher auf den umgekehrten Effekt, daß nämlich die fehlende Genehmigungskompetenz des (baden-württembergischen) Pendants mit höheren fachlichen Anforderungen einhergeht (gleichsam im Sinne eines Kompensierens jenes Mangels). ${ }^{91}$

Unabhängig von den im einzelnen unterschiedlichen Anteilen, die jeweils auf die vor bzw. erst nach offizieller Antragstellung geforderten Prüfbelege und Meßnachweise entfallen, ergibt der Vergleich der beiden Zeiträume, daß bei beiden Genehmigungsmodellen - wenn auch in unterschiedlichem Maße - der Schwerpunkt der Abklärung der materiellen Genehmigungsvoraussetzungen im Vorantragszeitraum liegt. Das gilt zumindest für die i.e.S. immissionsschutzrechtlich relevante Beschaffenheit der Anlage und ihres Betriebs. ${ }^{92}$

Auch der angesprochenen Fristsetzungen für Sanierungsmaßnahmen bzw. -konzepte (Kategorie b) bediente man sich beim LRA-1 mit einem Anteil von $23,1 \%$ der Beratungsfälle häufiger als beim GAA-N mit einem entsprechenden Anteil von lediglich 8,3\%.$^{93}$ In dieser Differenz spiegeln sich allerdings auch die unterschiedlich hohen Anteile an Anlagen wider, die beide Ämter jeweils als (immissionsschutzrechtlich) sanierungsbedürftig eingestuft hatten. ${ }^{94}$

Im Gebrauch der übrigen Instrumente zeigen die Behörden eine weitgehend ähnliche Praxis. Fristen für die Antragseinreichung setzte das LRA-1 in einem Drittel $(33,3 \%)$ aller Beratungsfälle, beim GAA-N fällt der entsprechende Anteil mit 29,1\% etwas geringer aus. Auf der anderen Seite scheint es dem GAA-N eher gelungen zu sein, seinen Fristsetzungen auch

$90 \mathrm{chi}^{2}=1,72<2,71$ für $\mathrm{p}=0,1$ und $\mathrm{df}=1$. - Weitere Differenzierungen zur Häufigkeit von Gutachten finden sich unten Ziff. 2.4.4 mit Tab. 19 und Ziff. 3.1.2.

$91 \mathrm{Vgl}$. hierzu auch näher unten Ziff. 3.3.2.

92 Für die nicht-immissionsschutzrechtlichen Forderungen sonstiger Fachbehörden gilt das nicht. Vgl. zu den hier nur knapp umrissenen Zusammenhängen ausführlicher unten Ziff. 2.4.4.

$93 \mathrm{chi}^{2}=3,75>2,71, \mathrm{p}=0,1, \mathrm{~d} \mathbf{f}=1$. 
den entsprechenden Respekt auf seiten des Betreibers zu verschaffen. Dort wurden in neun von 14 Fällen, also zu knapp zwei Dritteln, die Fristen eingehalten, beim LRA-1 in sechs von 13 Fällen, also nur knapp zur Hälfte. ${ }^{95}$ Die im Norden günstigere Relation verdankt sich weniger dem Umstand, daß die Fristsetzungen öfter frühzeitig mit möglichem Verwaltungszwang verbunden worden waren, als vielmehr dem Umstand, daß der Betreiber zur Fristwahrung öfter um eine Fristverlängerung nachgesucht (und diese auch erhalten) hatte als im Süden. Allerdings läßt sich die Bitte um Fristverlängerung auch dahingehend interpretieren, daß der Betreiber die Frist als verbindlich erachtet.

Um die Durchsetzungschancen gegenüber dem Betreiber zu steigern, verbanden die Behörden ihre Forderungen zum Teil mit der (förmlichen) ${ }^{96}$ Androhung von Verwaltungszwang im Falle der Nichterfüllung, beim GAA-N in $14,6 \%$ und beim LRA-1 in $15,3 \%$ aller Beratungsfälle. ${ }^{97}$ Beim GAA-N wurden vier der sieben angedrohten Zwangsmaßnahmen vollstreckt, beim LRA-1 nur eine von sechs. ${ }^{98}$ Dabei ist bemerkenswert, daß das LRA-1 in vier der übrigen fünf einschlägigen Fälle von der angekün-

94 Entsprechend der Erhebungsgrundlage der vorliegenden Untersuchung handelt es sich um Sanierungsfälle, in denen die notwendigen Um- bzw. Nachrüstungen eine »wesentliche« Anlagenänderung i.S.d. § $15 \mathrm{BImSchG} \mathrm{darstellten} \mathrm{und} \mathrm{entsprechend}$ eine Änderungsgenehmigung erforderten. Die Zahl dieser Fälle bzw. ihr Anteil an der Gesamtheit der Verfahren beläuft sich beim GAA-N auf $8(=13,6 \%)$, beim LRA1 auf $11(=25,6 \%)$. Die Differenz bleibt allerdings unterhalb statistischer Signifikanz $\left(\right.$ chi $^{2}=2,25<2,71$ für $p=0,1$ und $\mathrm{df}=1$.). Beim LRA-2 beträgt der entsprechende Anteil 3 Fälle $(=12,5 \%)$.

95 Beim LRA-2 verhält es sich mit drei von sieben Fällen ähnlich wie beim LRA-1.

96 Bei förmlichen Ankündigungen von Verwaltungszwangsmaßnahmen wird dem Adressaten entsprechend den Bestimmungen des jeweiligen Landesverwaltungsgesetzes eine Äußerungsmöglichkeit eingeräumt. Von solchen förmlichen Ankündigungen sind bloße Hinweise der Behörde etwa auf die Rechtswidrigkeit (und ggf. auch Strafbarkeit) des fortgesetzten Betriebs einer nicht genehmigten oder genehmigungswidrig betriebenen Anlage oder Hinweise auf die Möglichkeit der Betriebsuntersagung, der Stillegungsverfügung oder des Widerrufs der bestehenden Genehmigung (nach $\S \S 20,21 \mathrm{BImSchG)} \mathrm{zu} \mathrm{unterscheiden.} \mathrm{Die} \mathrm{(rechtliche)} \mathrm{Unverbind-}$ lichkeit solcher Hinweise wurde offenbar von den Betreibern erkannt, sie ließen sich von ihnen jedenfalls kaum beeindrucken.

97 Bezieht man die Androhungen von Verwaltungszwang auf die Gesamtzahl der Beratungsfälle, die von mindestens einer der in Tab. 11 aufgelisteten Maßnahmen betroffen waren (Kategorie f), ergibt sich beim GAA-N mit 33,3\% ein (allerdings statistisch nicht signifikant) höherer Anteil als beim LRA-1 mit 22,2\%.

98 Aus Vergleichsgründen bleibt eine beim LRA-1 erlassene nachträgliche Anordnung unberücksichtigt, da diese auf Grund eines Ministerialerlasses zur Durchführung der Sanierung von Flüssiggasaltanlagen zwingend vorgeschrieben war, und zwar auch in den Fällen, in denen der Betreiber die neuen, abgesenkten Grenzwerte der TALuft faktisch bereits einhielt, seine immissionsschutzrechtliche Genehmigung aber noch auf den alten, höheren Werten basierte. 
digten Sanktion absah, obwohl die (nach vorangegangener, teils wiederholter Anmahnung) gesetzte »letzte« Frist erneut überschritten worden war. Lediglich in einem Fall hatte die Sanktionsandrohung den gewünschten Erfolg.

Die einzige beim LRA-1 vollstreckte Zwangsmaßnahme bestand in der Untersagung des Weiterbetriebs bis zur Erteilung der Änderungsgenehmigung. Die vier beim GAA-N vollstreckten Maßnahmen betrafen in zwei Fällen nachträgliche Anordnungen (nach $\S 17 \mathrm{BImSchG),} \mathrm{in} \mathrm{den} \mathrm{anderen}$ beiden Fällen die Verhängung eines Bußgelds (nach dem $\mathrm{OWiG}$ ) wegen (fortgesetzten) ungenehmigten Betriebs. Die beiden nachträglichen Anordnungen wurden allerdings im nachhinein zugunsten des Betreibers abgemildert. Von den beiden Bußgeldbescheiden resultiert lediglich einer (in Höhe von DM 5.000,-) aus einer autonomen Entscheidung der Genehmigungsbehörde. Der andere (in Höhe von DM 200,-) erging gegen deren Willen auf Anordnung der Bezirksregierung, die damit einem entsprechenden Verlangen der Baubehörde zur Befriedung eines internen Behördenstreits nachkam.

Zusammengefaßt ergibt sich, daß das verwaltungsrechtliche Repertoire zur (effektiven) Durchsetzung behördlicher Forderungen bei der badenwürttembergischen, verwaltungsversierten Genehmigungsbehörde keine größere Rolle spielt als bei der niedersächsischen, primär technisch qualifizierten Genehmigungsbehörde. Die Befunde erwecken vielmehr den Eindruck (der sich aber wegen der geringen Fallzahlen nicht absichern läßt), daß die Techniker in dieser Hinsicht etwas couragierter vorgehen als die Verwaltungsfachleute. Ob dies eher auf die unterschiedliche fachliche Qualifikation oder eher auf das unterschiedliche Maß (kommunal-)politischer Verflechtung zurückzuführen ist, ist eine Frage, die hier nicht entschieden werden kann.

\section{Antragsbearbeitung (äußerlicher Verfahrensverlauf)}

Die Leitung des Verfahrens und damit die Verantwortung für dessen Ablauf bzw. Durchführung bis zur abschließenden Entscheidung über die Genehmigungserteilung obliegen nach dem niedersächsischen wie auch nach dem baden-württembergischen Modell der Genehmigungsbehörde. Im einzelnen sind ihr damit im wesentlichen folgende, den Verfahrensablauf regelnde Aufgaben zugewiesen: ${ }^{99}$ Vollständigkeitsprüfung der Antragsunterlagen (2.1), Informationspflichten gegenüber dem Antragsteller (2.2), Beteiligung der in ihren Belangen mutmaßlich betroffenen Fachbehörden und

99 Niedergelegt in der 9 . BImSchV. 
sonstigen Stellen (2.3), Organisation und Koordination der Kooperation zwischen den Verfahrensbeteiligten (2.4).

\subsection{Vollständigkeitsprüfung der Antragsunterlagen}

Nach den gesetzlichen Vorschriften hat die Genehmigungsbehörde den Genehmigungsantrag samt Unterlagen nach Eingang auf Vollständigkeit im Hinblick auf die für die behördliche Entscheidung erforderlichen Angaben zur Anlage und zum Anlagenbetrieb zu prüfen. ${ }^{100}$ Dieser Aufgabe gerecht zu werden, dürfte (reinen) Verwaltungsfachleuten schwerer fallen als ausgewiesenen Technikern. Insofern ist es nicht überraschend, daß - wie Tab. 12 zeigt - das GAA-N bei (anteilig) doppelt so vielen Anträgen (19 = $32,8 \%$ ) Unvollständigkeit der Antragsunterlagen feststellte und vom Antragsteller entsprechende Ergänzungen forderte ${ }^{101}$ wie das LRA-1 (7 = $16,3 \%) .{ }^{102}$ Nur in der Minderzahl wurde den Ergänzungsforderungen durch Fristsetzung Nachdruck verliehen, beim LRA-1 sogar noch weniger als beim GAA-N. ${ }^{103}$

$100 \S 7$ Abs. 1 i.V.m. $\S \S 4$ ff. der 9. BImSchV.

101 Dabei forderte das GAA-N in zwei Fällen, in denen keine Vorantragsberatung vorausgegangen war, auch die Vorlage von gutachtlichen Prüfbelegen bzw. Meßnachweisen. Vgl. auch unten Ziff. 2.4.4 u. 3.1.2.

102 Die Differenz ist signifikant $\left(\mathrm{chi}^{2}=3,55>2,71, \mathrm{p}=0,1\right.$, df $\left.=1\right)$. - Beim LRA-2 liegt mit $9(=37,5 \%)$ einschlägigen Fällen der Anteil sogar noch über dem des GAAN, was auf die erwähnte (Anm. 53) atypische Beziehung zwischen dem LRA-2 und dem zugehörigen GAA zurückzuführen sein dürfte. Speziell im vorliegenden Zusammenhang klärte der dortige LRA-Sachbearbeiter auf »kurzem« Wege - telefonisch oder im Rahmen der regelmäßigen informellen persönlichen Begegnungen mit dem zuständigen GAA-Sachbearbeiter die Vollständigkeitsfrage im Sinne einer ersten Plausibilitätsprüfung ab, bevor er dann den - ggf. bereits vervollständigten - Antrag dem GAA formell zur Prüfung und Stellungnahme zuleitete.

103 Der Unterschied ist allerdings statistisch nicht signifikant: chi $^{2}=0,97$ (Yates' Korrektur) $<2,71$ für $\mathrm{p}=0,1$ und $\mathrm{df}=1$. 
Tab. 12

Nachforderungen von Unterlagen im Rahmen der Vollständigkeitsprüfung (vor Fachbehördenbeteiligung ${ }^{*}$ )

\begin{tabular}{|l|c|c|c|}
\cline { 2 - 4 } \multicolumn{1}{c|}{} & $G A A-N$ & $L R A-1$ & $L R A-2$ \\
\hline Verfahren mit Nachforderungen & 19 & 7 & 9 \\
[darunter: mit Fristsetzung] & $32,8 \%$ & $16,3 \%$ & $37,5 \%$ \\
\hline Verfahren ohne Nachforderungen & {$[8]$} & {$[1]$} & {$[3]$} \\
\hline \hline Verfahren gesamt & 39 & 36 & 15 \\
& $67,2 \%$ & $83,7 \%$ & $62,5 \%$ \\
\hline \hline
\end{tabular}

* Im Süden unter Einschluß des zuständigen GAA.

\subsection{Unterrichtung des Antragstellers über den Gang des Verfahrens}

Die Genehmigungsbehörde ist aủsdrücklich dazu verpflichtet, dem Antragsteller den Eingang seines Antrags schriftlich zu bestätigen ${ }^{104}$. Ferner hat sie ihn, sobald die Antragsunterlagen vollständig sind, über die voraussichtlich zu beteiligenden Behörden und den geplanten zeitlichen Ablauf des Verfahrens zu unterrichten. ${ }^{105}$ Der Zeitpunkt der Vollständigkeit der Antragsunterlagen markiert den Beginn der im BImSchG festgesetzten Frist, innerhalb derer die Entscheidung über den Antrag i.d.R. zu ergehen hat, ${ }^{106}$ und damit den Beginn der »offiziellen « Verfahrenslaufzeit. ${ }^{107}$

Die Anleitung des Verwaltungshandelns durch Formvorschriften wie die hier genannten ist ein klassisches Merkmal von Bürokratien. Es liegt von daher nahe zu erwarten, daß das auf den Umgang mit dem verwaltungsrechtlichen Instrumentarium geschulte Personal des baden-württembergischen Modells auf die Wahrung der Formvorschriften größeres Gewicht legt als das auf die Regelung technischer Sachverhalte ausgerichtete Personal des niedersächsischen Modells. Verwaltungsleute sind sich auf Grund ihrer Vorbildung in höherem Maße der möglichen rechtlichen Konsequen-

$104 \S 6$ der 9. BImSchV.

$105 \S 7$ Abs. 2 der 9. BImSchV.

106 Für vereinfachte Verfahren beträgt diese Regelfrist drei Monate für Neugenehmigungen ( $§ 10$ Abs. 6a Satz 1) und sechs Monate für Änderungsgenehmigungen ( $\$ 15$ Abs. 1 Satz 2). Zu den unterschiedlichen Fristen für die beiden Genehmigungsarten vgl. die Bemerkungen unten Ziff. 4.1.

107 Zur Differenzierung verschiedener Verfahrensdauern s. unten Ziff. 4.2.1. 
zen ihres Handelns bewußt und geneigt, sich in dieser Hinsicht abzusichern, so könnte man weiter vermuten, während Techniker ihr Handeln in erster Linie an fachtechnischen Kriterien der zu bewältigenden Probleme ausrichten.

Die Untersuchungsbefunde stützen diese These nur bedingt. Einerseits erfüllte das LRA-1 die Pflicht der Bestätigung des Antragseingangs tatsächlich in größerem Umfang als das GAA-N, nämlich in $40(=93 \%)$ gegenüber $46(=79,3 \%)$ der Verfahren. ${ }^{108}$ Ähnliches gilt für die Wahrung der Formvorschrift, dem Antragsteller den Zeitpunkt der (offiziellen) Einleitung des Verfahrens mitzuteilen. Eine solche (schriftliche) Mitteilung erging beim LRA-1 in 93\% der Verfahren, beim GAA-N nur in 67,2\%. ${ }^{109}$ Nimmt man jedoch - andererseits - den inhaltlichen Informationsgehalt hinzu und bezieht ihn auf die gesetzlichen Anforderungen, die ja sowohl eine Unterrichtung über die Vollständigkeit der Unterlagen als auch über die voraussichtlich zu beteiligenden Behörden und den geplanten zeitlichen Ablauf des Genehmigungsverfahrens vorsehen, ergibt sich ein anderes Bild. Im Süden wie im Norden zeigten sich die Behörden wenig geneigt, sich in diesen Punkten festzulegen. Sie bevorzugten statt dessen - meist in Form von Textbausteinen verfügbare - inhaltlich unverbindliche Standardfassungen. Danach behielt man sich in der Frage der Vollständigkeit der Antragsunterlagen Nachforderungen vor, wies auf die Beteiligung von »in ihren Belangen betroffenen Fachbehörden« zwar hin, ohne diese namentlich zu nennen, und ließ den Antragsteller wissen, man würde ihn über den weiteren Verfahrensablauf $» z u$ gegebener Zeit« unterrichten. Von solchen Standardformulierungen abweichende Schreiben mit verbindlicher Auskunft waren dabei eher beim GAA-N als beim LRA-1 anzutreffen. Das gilt sowohl für die Bestätigung der Vollständigkeit der Unterlagen ${ }^{110}$ als auch für die namentliche Auflistung der zu beteiligenden Fachbehörden. ${ }^{11}$ Zeitliche Festlegungen wie etwa das Inaussichtstellen der Genehmigungserteilung innerhalb einer bestimmten Frist waren nur beim GAA-N zu beobachten, wenn auch lediglich in wenigen (5) Fällen. Daß die angekündigte Frist

108 Der Unterschied ist signifikant $\left(\mathrm{chi}^{2}=3,70>2,71, \mathrm{p}=0,1, \mathrm{df}=1\right)$. Formvollendet in dieser Beziehung verhielt sich das LRA-2 mit 100\% Eingangsbestätigungen.

109 Der Unterschied ist signifikant $\left(\mathrm{chi}^{2}=9,73>7,88, \mathrm{p}=0,005, \mathrm{df}=1\right)$. Beim LRA2 liegt der Anteil bei 95,8\% aller Verfahren.

110 Ausdrückliche Vollständigkeitsbestätigungen finden sich beim GAA-N in sechs Verfahren $(=10,3 \%$ v. 48$)$, beim LRA-1 in keinem einzigen; der Unterschied ist signifikant $\left(\mathrm{chi}^{2}=3,88\right.$ [Yates' Korrektur] $>3,84, \mathrm{p}=0,05, \mathrm{df}=1$,). LRA-2 weist 4 einschlägige Fälle $(=16,7 \%)$ auf und liegt damit über dem Anteil beim GAA-N.

111 GAA-N in acht $(=13,8 \%$ v. 48$)$, LRA- 1 in drei $(=7,0 \%$ v. 48$)$, LRA- 2 in $23(=95,8 \%$ v. 24) Fällen. Während beim GAA-N und LRA-1 die Information über die beteiligten Fachbehörden im abschließenden Genehmigungsbescheid gleichsam nachgeholt wurde, unterblieb dies beim LRA-2, offenbar die bereits erbrachte »Vorleistung « berücksichtigend. 
in keinem Fall eingehalten, vielmehr um mindestens einen Monat überschritten wurde, zeigt, wie riskant derartige Festlegungen oder $\gg$ Versprechungen « für die Behörde sind. Es dürfte eben diese Erfahrung sein, die es ratsam erscheinen ließ, selbstverpflichtende Auskünfte gegenüber dem Antragsteller zu vermeiden.

Im Ergebnis zeigt sich, daß die Techniker im Norden im großen und ganzen einen nicht minder reflektierten, mögliche Konsequenzen mitbedenkenden Umgang mit den für ihren Handlungsbereich einschlägigen Verwaltungsvorschriften pflegten wie die Verwaltungsfachleute. Dies dürfte nicht nur ihrer (wenn auch eher rudimentären) verwaltungsrechtlichen Zusatzausbildung zuzuschreiben sein, sondern auch den Sozialisationseffekten eines learning by doing.

Statt für eine gewisse Transparenz des weiteren Verfahrensgangs entsprechend den angesprochenen Formvorschriften zu sorgen, die dem Antragsteller zur Wahrung seiner Rechte und Interessen von Anbeginn an entgegenkäme, bevorzugen die Genehmigungsbehörden in beiden Modellen (nach Lage der Akten) eine ihn in dieser Beziehung eher im Ungewissen haltende (insoweit »obrigkeitsstaatlich « anmutende) Verwaltungspraxis. Der Betreiber muß schon von sich aus aktiv werden und an die Behörde herantreten, um sein Interesse an frühzeitiger Unterrichtung geltend zu machen, was naheliegenderweise größeren Betricben leichter fällt (und ihnen auch leichter gemacht wird) als kleineren.

Speziell dadurch, daß die Behörden eine förmliche Bestätigung der Vollständigkeit der Antragsunterlagen in der Regel vermeiden, verschaffen sie sich einen vom Gesetz nicht gedeckten Handlungsspielraum. Wird dieser Zeitpunkt nicht fixiert, ist die Einhaltung der erwähnten gesetzlichen Regelfrist, innerhalb derer das Verfahren zum Abschluß zu bringen ist, für den Antragsteller (wie auch für die vorgesetzten behördlichen Stellen) nur schwer zu kontrollieren. Das stellt auch eine empirische Untersuchung vor das Problem, den im Gesetz definierten Verfahrensbeginn zu ermitteln, um ihn als Maßstab für die tatsächliche Dauer zu verwenden (s. dazu näher unten Ziff. 4.2.1).

\subsection{Beteiligung von Fachbehörden und sonstigen Stellen}

Die nicht nur im immissionsschutzrechtlichen Genehmigungsverfahren, sondern auch andernorts wie etwa beim Baugenehmigungsverfahren vorgeschriebene Beteiligung anderer Fachbehörden soll sicherstellen, daß die von diesen vertretenen öffentlichen Belange durch eine entsprechende Stellungnahme bei der Genehmigungsentscheidung Berücksichtigung finden. Äußern sich die angesprochenen Behörden nicht binnen der gesetzlich vorgegebenen Frist von einem Monat, kann die Genehmigungsbehörde davon 
ausgehen, daß diese von ihrem Recht keinen Gebrauch machen wollen. ${ }^{112}$ Sie kann daraus allerdings nicht den Schluß ziehen, daß der jeweilige Belang durch das Vorhaben nicht berührt ist. Sie muß in diesem Falle vielmehr selbst die inhaltliche Entscheidung treffen (und verantworten), was ggf. auch heißen kann, daß sie dazu eigene Ermittlungen anstellt. ${ }^{113}$ Formalrechtlich ist die fachbehördliche Stellungnahme für die Genehmigungsbehörde nicht bindend, ihr Übergehen oder Abändern birgt aber das Risiko einer rechtswidrigen Ermessensausübung in sich. Entsprechend wird ein solches Vorgehen in der Regel gemieden. ${ }^{114}$ Die Fachbehörden ihrerseits können praktisch keinen Einfluß auf eine Beteiligung nehmen. Abgesehen davon, daß es ihnen an einem eigenen Rechtsanspruch auf Beteiligung mangelt, fehlt es ihnen i.d.R. auch an Informationen über bei anderen Stellen laufende Verfahren. Nur soweit sie bei Gelegenheit davon erfahren, können sie auf eine Beteiligung hinwirken - angesichts des bei der Genehmigungsbehörde liegenden Risikos ihrer Nichtbeteiligung mit guten Erfolgsaussichten.

In den beiden Organisationsmodellen ist der Spielraum, den die Genehmigungsinstanz bei der Auswahl der im einzelnen zu hörenden Fachbehörden hat, unterschiedlich zugeschnitten, soweit diese Teil der Kommunalverwaltung sind. Die hier untersuchten, in die Kreisverwaltung integrierten baden-württembergischen Umweltämter entscheiden auch über die Beteiligung der übrigen dort angesiedelten Dienststellen. Dazu gehören insbesondere die hier interessierenden unteren Behörden für Baurecht, Wassserrecht, Naturschutzrecht, aber auch die Abfallrechtsbehörde und der vorbeugende Brandschutz. ${ }^{115}$

Anders verhält es sich beim niedersächsischen GAA als einer von der Kommunalverwaltung separierten staatlichen Sonderbehörde. Dieses wendet sich hinsichtlich der im konkreten Fall betroffenen fachlichen Belange, soweit sie von den Kommunen verwaltet werden, auf dem Dienstweg an die

$112 \S 11$ i.V.m. $\S 24$ der 9. BImSchV. - Eine rechtliche Verpflichtung der Fachbehörden, eine Stellungnahme abzugeben, besteht nicht. Im übrigen verfügt die Genehmigungsbehörde gegenüber den Fachbehörden über keinerlei Weisungsbefugnis.

113 Vgl. die Kommentierung bei Hansmann 1994, S. 229, Fn. 29.

114 Davon zu unterscheiden sind Versuche der Genehmigungsbehörde, eine Fachbehörde zur Rücknahme oder zu Abänderungen von Auflagen (fast immer zugunsten des Betreibers) zu bewegen (s. dazu unten Ziff. 3.1.3, ferner auch Ziff. 3.2 und Ziff. 3.4.2). Bleibt ein entsprechender Erfolg aus, fügt sich dem die Genehmigungsbehörde in der Regel. Abänderungen von Auflagen gegen das Votum der Fachbehörde bilden im Süden wie im Norden einen äußerst seltenen Ausnahmefall.

115 Lediglich bei Großen Kreisstädten mit eigener Baurechtsbehörde entfällt die Zuständigkeit der Kreisverwaltung für die baurechtlichen Belange. 
zuständige Kreis- bzw. Stadtverwaltung, ${ }^{116}$ die ihrerseits darüber entscheidet, welche Fachämter sie zur Stellungnahme auffordert. Für das GAA bedeutet dies einerseits eine Entlastung von Koordinationsarbeit, wie sie das baden-württembergische LRA zu erbringen hat. Andererseits bedeutet es eine faktische Beschneidung seines Entscheidungsspielraums. ${ }^{117}$ Zwar kann es sich »auf dem kleinen Dienstweg « auch direkt mit einem Amt der Kommunalverwaltung in Verbindung setzen. Es wird dies aber nur dann tun, wenn es aus eigenem Interesse einen spezifischen fachlichen Belang berücksichtigt haben will und die Verantwortung für eine eigene Entscheidung auf fachfremdem Gebiet nicht übernehmen möchte. Aber auch dann wird es von der internen Organisation (Kontrollstruktur) der jeweiligen Kommunalverwaltung abhängen, wieweit auch solche Kontakte über die zentrale Stelle ablaufen.

Die Frage ist, ob sich die in dieser Weise unterschiedlich vorstrukturierten Handlungsspielräume hinsichtlich der Fachbehördenbeteiligung auf die Beteiligungspraxis auswirken. Dies gilt einmal im Hinblick auf die Zahl der in den Verfahren beteiligten Fachbehörden (Ziff. 2.3.1) und zum anderen im Hinblick auf die Art der jeweils berücksichtigten fachlichen Belange (Ziff. 2.3.2). Aus Vergleichsgründen nicht mitgezählt ist dabei das GAA als Fachbehörde, das im Süden wie im Norden stets beteiligt war, im Norden aber eben in »Personalunion « mit der Genehmigungsbehörde.

\subsubsection{Umfang der Fachbehördenbeteiligung}

Einen Überblick über die Anzahl der in den Genehmigungsverfahren jeweils beteiligten Fachbehörden gibt Tab. 13.1 in Form einer Häufigkeitsverteilung auf verschiedene Größenklassen.

Wie zu sehen ist, bewegen sich die prozentualen Abweichungen zwischen GAA-N und LRA-1 in den einzelnen Klassen unterhalb der 10\%Marke (und unterhalb statistischer Signifikanz). Die einzige Ausnahme bildet die Gruppe von Verfahren mit fünf beteiligten Fachbehörden, die einen Anteil von $22,4 \%$ beim GAA-N, aber von nur $4,7 \%$ beim LRA-1 ausmachen. ${ }^{118}$ Im übrigen variieren bei beiden Behörden die Einzelhäufigkeiten

116 Als für immissionsschutzrechtliche Genehmigungsverfahren zuständige »Zentrale« fungiert bei den Kreisverwaltungen in der Regel das Ordnungsamt, bei den kreisfreien Städten mit stärkerer fachlicher Binnendifferenzierung das hauseigene Umweltamt (aber ohne Genehmigungskompetenz für die hier untersuchten Verfahren).

117 Die rechtliche Verantwortung für die Nicht-Beteiligung einzelner Fachämter liegt auch in diesem Fall allein beim GAA als zuständiger Genehmigungsbehörde. Im Zweifel muß es über die Betroffenheit der fachlichen Belange eines von der kommunalen »Zentrale« nicht einbezogenen Fachamts selbst entscheiden.

118 Der Unterschied ist (als einziger) statistisch signifikant $\left(\right.$ chi $^{2}=6,17>5,02, \mathrm{p}=$ 0,025 , df $=1$ ). 
diskontinuierlich über die verschiedenen Größenklassen, d.h. es ist weder eine Tendenz zu einer gleichmäßigen Verteilung noch eine Tendenz zu einer kontinuierlichen $\mathrm{Zu}$ - oder Abnahme der Anteile bei zunehmender Klassengröße erkennbar. Zudem verlaufen die Diskontinuitäten bei den beiden Behörden teilweise in entgegengesetzten Richtungen mit kompensatorischen Effekten in einzelnen Größenklassen. ${ }^{119}$

\section{Tab. 13.1}

Anzahl der in den Genehmigungsverfahren beteiligten Fachbehörden* (Häufigkeitsverteilung)

\begin{tabular}{|l|c|c|c|}
\cline { 2 - 4 } \multicolumn{1}{c|}{} & GAA-N & $L R A-1$ & $L R A-2$ \\
\hline \multirow{2}{*}{ Keine } & 2 & 0 & 0 \\
& $3,4 \%$ & $0,0 \%$ & $0,0 \%$ \\
\hline \multirow{2}{*}{ Eine } & 0 & 0 & 0 \\
& $0,0 \%$ & $0,0 \%$ & $0,0 \%$ \\
\hline \multirow{2}{*}{ Drei } & 0 & 3 & 0 \\
& $0,0 \%$ & $7,0 \%$ & $0,0 \%$ \\
\hline Vier & 8 & 6 & 3 \\
\hline Fünf & $13,8 \%$ & $14,0 \%$ & $12,5 \%$ \\
\hline \multirow{2}{*}{ Sechs } & 14 & 14 & 9 \\
\hline Sieben und mehr** & $24,1 \%$ & $32,6 \%$ & $37,5 \%$ \\
\hline \hline Verfahren gesamt*** & 13 & 2 & 6 \\
\hline \hline
\end{tabular}

Aus Vergleichsgründen im Süden ausgenommen GAA.

** Beim GAA-N und LRA-1 jeweils maximal 12, beim LRA-2 zehn Behörden/Stellen.

*** Abweichungen von 100,0\% durch Rundungen bei den prozentualen Einzelhäufigkeiten.

119 Dies schlägt sich in einer statistisch signifikanten Differenz der Gesamtverteilungen nieder $\left(\mathrm{chi}^{2}=12,81>12,6, \mathrm{p}=0,05, \mathrm{df}=6\right)$. Weitergehende inhaltliche Schlußfolgerungen im Hinblick auf die Praxis der Fachbehördenbeteiligung lassen sich daraus aber nicht ziehen. 
Dies tritt anschaulich hervor, wenn man die Größenklassen zu drei Gruppen bündelt, eine erste, die die Fälle mit bis zu drei, eine zweite, die vier bis sechs und eine dritte, die (wie bisher) sieben und mehr beteiligte Fachbehörden umfaßt (Tab. 13.2).

Danach ging im Norden wie im Süden die Mehrzahl der Verfahren mit einer Beteiligung von vier bis sechs Fachbehörden einher (mittlere Gruppe). Dabei beläuft sich der entsprechende Anteil beim LRA-1 mit $55,8 \%$ auf etwas mehr als die Hälfte, beim GAA-N mit 69,0\% sogar auf mehr als zwei Drittel. Der Unterschied bleibt allerdings unterhalb statistischer Signifikanz. ${ }^{120}$ Die übrigen Verfahren verteilen sich bei beiden Behörden zu näherungsweise ähnlichen Anteilen auf die anderen beiden Gruppen. Auf die erste Gruppe mit bis zu drei beteiligten Fachbehörden entfallen bei beiden Ämtern jeweils etwa ein Fünftel aller Verfahren (beim GAA-N mit 17,2\% etwas weniger und beim LRA-1 mit 20,9\% etwas mehr). Stärker, aber gleichfalls nicht statistisch signifikant, differieren die Anteile bei der dritten Gruppe mit sieben und mehr beteiligten Fachbehörden mit $23,3 \%$ beim GAA-N und $13,8 \%$ beim LRA-1.

Tab. 13.2

Anzahl der in den Genehmigungsverfahren beteiligten Fachbehörden* (komprimierte Häufigkeitsverteilung)

\begin{tabular}{|l|c|c|c|}
\cline { 2 - 4 } \multicolumn{1}{c|}{} & GAA-N & LRA-1 & LRA-2 \\
\hline Bis zu drei & 10 & 9 & 3 \\
& $17,2 \%$ & $20,9 \%$ & $12,5 \%$ \\
\hline Vier bis sechs & 40 & 24 & 18 \\
& $69,0 \%$ & $55,8 \%$ & $75,0 \%$ \\
\hline Sieben und mehr** & 8 & 10 & 3 \\
\hline \hline Verfahren gesamt & $13,8 \%$ & $23,3 \%$ & $12,5 \%$ \\
\hline \hline
\end{tabular}

* Aus Vergleichsgründen im Süden ausgenommen GAA.

* Beim GAA-N und LRA-1 jeweils maximal 12, beim LRA-2 zehn Behörden/Stellen.

Insgesamt bleiben die Abweichungen in der Verteilung der Verfahren auf die einzelnen Größenklassen der Fachbehördenbeteiligung unterhalb eines statistisch relevanten Niveaus. Von daher läßt sich von einer vergleichbaren Beteiligungspraxis bei den beiden Genehmigungsmodellen sprechen. Diese

120 chi $^{2}=1,84<2,71$ für $\mathrm{p}=0,1$ und $\mathrm{df}=1$. 
Schlußfolgerung läßt sich durch die Analyse der zentralen statistischen Parameter zusätzlich absichern (Tab. 13.3).

Durchschnittlich wurden beim GAA-N 5,0 und beim LRA-1 5,1 Fachbehörden je Verfahren beteiligt. ${ }^{121}$ Die häufigste Konstellation ist bei beiden Behörden die Beteiligung von vier Fachbehörden (Modalwert) ${ }^{122}$, die maximale Beteiligungszahl in ein und demselben Verfahren liegt bei jeweils 12. In zwei Hinsichten sind allerdings Unterschiede festzustellen. Der eine betrifft die Zahl der in einem Verfahren mindestens beteiligten Fachbehörden. Während beim LRA-1 in sämtlichen Verfahren Fachbehörden (und zwar mindestens zwei) beteiligt wurden, kamen beim GAA-N auch (und zwar zwei) Fälle ohne jegliche Fachbehördenbeteiligung vor. In den übrigen Verfahren waren mindestens drei externe Behörden präsent. Der zweite Unterschied betrifft den Median, der beim LRA-1 bei 4 und beim GAA-N bei 5 liegt. Das bedeutet, daß sich beim LRA-1 die (untere) Hälfte der Verteilung auf Verfahren mit nicht mehr als vier Fachbehörden erstreckt, während beim GAA-N diese Hälfte auch noch Verfahren mit fünf Fachbehörden einschließt. Beide Unterschiede, die die Spannweite der Verteilung bzw. ein $\mathrm{Ma} \beta$ der zentralen Tendenz berühren, sind aber zu gering, als daß sie sich in einer signifikanten Differenz der Varianzen (Streuung der Einzelwerte der Gesamtverteilung um den Mittelwert) niederschlügen. ${ }^{123}$

Die gesetzlich vorgeschriebene Fachbehördenbeteiligung führt, so das Resümee, in beiden Modellen zu einer im Durchschnitt gleich großen »Belastung « - wenn man aus Gründen der Vergleichbarkeit im vorliegenden Zusammenhang das im Süden zusätzlich hinzukommende GAA unberücksichtigt läßt. Demnach hat speziell auch der Umstand, daß das niedersächsische GAA-N organisationsbedingt die Entscheidung in der Beteiligungsfrage zu einem guten Teil an die »Zentrale « der allgemeinen Verwaltung abgibt, während die baden-württembergische Genehmigungsbehörde selbst als eine solche »zentrale Koordinationsstelle « fungiert, keine Auswirkungen auf den Beteiligungsumfang. Die Kommunalverwaltungen sind offenbar unabhängig davon, ob sie über eine Genehmigungskompetenz verfügen, im Norden wie im Süden gleichermaßen darauf bedacht, daß die bei ihnen vertretenen fachlichen Belange auch Berücksichtigung finden. Das

121 Mittelwerttest und (vorgeschalteter) Varianztest ergeben auf jeweils hohem Signifikanzniveau (d.h bei Akzeptanz eines hohen Fehlerrisikos von 5\% je Seite der Verteilung für die Annahme von Ungleichheit) keine signifikante Differenz (Varianztest: $\mathrm{F}=1,26<\mathrm{F}_{(0,90 ; 42,57)}=1,41$; Mittelwerttest: $\left.\mathrm{t}=0,318<\mathrm{t}_{(0,90 ; 99)}=1,29\right)$. LRA-2 hebt sich von den beiden anderen Genehmigungsbehörden vor allem durch eine geringere Varianz ab, die Differenzen sind aber gleichfalls - wenn auch unter geringeren Fehlertoleranzen - nicht signifikant (Vergleich mit GAA-N: F = 1,77< $\mathrm{F}_{(0,95 ; 57,23)}=1,84$; Vergleich mit LRA-2: $\left.\mathrm{F}=2,24<\mathrm{F}_{(0,99 ; 42,23)}=2,44\right)$.

122 Vgl. auch die Häufigkeitsverteilung oben in Tab. 13.1.

123 Vgl. die in vorletzter Anm. wiedergegebenen Testwerte für die Varianzen. 
bestätigen auch die nachfolgenden Befunde zur Art der beteiligten fachlichen Belange.

Tab. 13.3

Anzahl der in den Genehmigungsverfahren beteiligten Fachbehörden* (statistische Parameter)

\begin{tabular}{|l|c|c|c|}
\cline { 2 - 4 } \multicolumn{1}{c|}{} & GAA- $N$ & LRA-1 & LRA-2 \\
\hline Arith. Mittel & 4,97 & 5,10 & 4,83 \\
\hline Varianz & 3,65 & 4,61 & 2,06 \\
\hline Median & 5,00 & 4,00 & 4,50 \\
\hline Modalwert & 4,00 & 4,00 & 4,00 \\
\hline Minimum & 0,00 & 2,00 & 3,00 \\
\hline Maximum & 12,00 & 12,00 & 9,00 \\
\hline \hline Verfahren gesamt & 58 & 43 & 24 \\
\hline
\end{tabular}

* Aus Vergleichsgründen im Süden ausgenommen GAA.

\subsubsection{Art der beteiligten fachliche Belange}

Nicht nur in quantitativer, sondern auch in qualitativer Hinsicht ergeben sich weitgehende Ähnlichkeiten der unter den beiden Genehmigungsmodellen jeweils vollzogenen Beteiligungspraxis. Dies zeigt eine Aufschlüsselung der in den Verfahren hauptsächlich berücksichtigten fachlichen Belange bzw. der am häufigsten herangezogenen Fachbehörden.

Wie aus Tab. 14 hervorgeht, differieren die jeweiligen Anteile zwischen GAA-N und LRA-1 um weniger als 10 Prozentpunkte. ${ }^{124}$ Davon auszunehmen sind zum einen die Abfallbehörden einschließlich der Abfallwirtschaftsämter, zum anderen die nicht den Fachbehörden i.e.S. zuzurechnenden Kommunen (s. dazu weiter unten).

124 Alle Verteilungsunterschiede bei den einzelnen Fachbehörden einschließlich den sonstigen Behörden zwischen GAA-N und LRA-1 sind statistisch nicht signifikant - ausgenommen die nachfolgend im Text genannten zwei Ausnahmen. Das gilt auch für die teilweise etwas größer ausfallenden Verteilungsunterschiede zwischen LRA1 und LRA-2. 
Die am häufigsten beteiligte Fachbehörde ist die untere Baurechtsbehörde (GAA-N zu 89,7\%, LRA-1 zu 90,7\%, entsprechend 1,0 Prozentpunkte Differenz), was daher rühren dürfte, daß nicht nur Neuerrichtungen, sondern auch die meisten Änderungen einer Anlage mit baulichen Maßnahmen verbunden sind. Es folgen die Fachbehörden für den (vorbeugenden) Brandschutz (81,0\% bzw. 72,1\%, entsprechend 8,9 Prozentpunkte Differenz), für Wasser- und Bodenschutz einschließlich der Wasserwirtschaftsämter $(89,7 \%$ bzw. 90,7\%, entsprechend 1,0 Prozentpunkte Differenz) sowie für den (allerdings deutlich weniger häufig herangezogenen) Naturschutz (29,3\% bzw. 23,3\%, entsprechend 6,0 Prozentpunkte Differenz). ${ }^{125}$

Tab. 14

Am häufigsten beteiligte Fachbehörden* (Auswahl)

\begin{tabular}{|l|c|c|c|}
\cline { 2 - 4 } \multicolumn{1}{c|}{} & GAA-N & LRA-1 & LRA-2 \\
\hline Baubehörde & 52 & 39 & 23 \\
& $89,7 \%$ & $90,7 \%$ & $95,8 \%$ \\
\hline \multirow{2}{*}{ Brandschutzbehörde } & 47 & 31 & 14 \\
& $81,0 \%$ & $72,1 \%$ & $58,3 \%$ \\
\hline Wasserschutzbehörde** & 52 & 39 & 24 \\
\hline Naturschutzbehörde & $89,7 \%$ & $90,7 \%$ & $100,0 \%$ \\
\hline \multirow{2}{*}{ Abfallbehörde*** } & 17 & 10 & 6 \\
\hline Sonstige Behörden & $29,3 \%$ & $23,3 \%$ & $25,0 \%$ \\
\hline Kommune & 43 & 3 & 8 \\
& $74,1 \%$ & $7,0 \%$ & $33,3 \%$ \\
\hline \hline Verfahren gesamt**** & 34 & 22 & 10 \\
& $58,6 \%$ & $51,2 \%$ & $41,7 \%$ \\
\hline \hline
\end{tabular}

* Aus Vergleichsgründen im Süden ausgenommen GAA.

** Einschließlich Wasserwirtschaft und Bodenschutz.

*** Einschließlich Abfallwirtschaft.

**** Da sich die Kategorien nicht wechselseitig ausschließen (Mehrfachnennungen), liegt die Summe der absoluten bzw. relativen Häufigkeiten über der Gesamtzahl der Verfahren bzw. über $100 \%$.

125 Beim LRA-2 fallen die Anteile vergleichbar aus. 
In gut der Hälfte der Genehmigungsverfahren (GAA-N zu 58,6\%, LRA-1 zu 51,2\%, entsprechend 7,4 Prozentpunkte Differenz) ${ }^{126}$ wurden eine oder mehrere sonstige Stellen hinzugezogen, die über eine breite Palette von weiteren öffentlichen Belangen streuen. Als Beispiele für die unter diese Kategorie subsumierten Behörden seien genannt: Gesundheitsamt, Veterinäramt, Amt für Denkmalschutz, Polizei, Bezirksschornsteinfeger, StraBenverkehrsamt, u.ä. Jeweils für sich genommen erfolgte ihre Beteiligung gegenüber den zuvor genannten, teilweise routinemäßig angehörten Fachbehörden erheblich seltener und dürfte in erster Linie je spezifischen Besonderheiten des Einzelfalls geschuldet gewesen sein.

Aus der weitgehend ähnlichen (qualitativen) behördlichen Beteiligungspraxis fällt die bereits als Ausnahme angesprochene Abfallbehörde heraus. Sie wurde beim GAA-N in 74,1\%, beim LRA-1 lediglich in 7,0\% (und beim LRA-2 in 33,3\%) der Verfahren beigezogen. Der Unterschied könnte sich daraus erklären, daß beim LRA-1 (wie auch beim LRA-2) die untere Abfallbehörde mit der (hier im Zentrum stehenden) unteren Immissionsschutzbehörde das Amt für Umweltschutz bilden. Die organisationsbedingte und auch räumliche Nähe (durch Ansiedlung auf demselben Flur) sorgt offenbar für einen hinreichenden Einblick der Sachbearbeiter des Immissionsschutzes in den Aufgabenbereich der Abfallbehörde, so daß diese nür in den Fällen (förmlich) beteiligt wurde, in denen abfallrelevante Belange auch tatsächlich betroffen waren. Demgegenüber scheint im Norden das überwiegend über die »Zentrale« der jeweils zuständigen Kommunalverwaltung laufende Beteiligungsverfahren eine routinemäßige Anfrage beim abfallrechtlichen Fachamt zu begünstigen. Als Indiz dafür läßt sich der Anteil der Fälle werten, in denen die Abfallbehörde keine Bedenken bzw. Auflagen gegen das in Frage stehende Vorhaben vorbrachte oder sogar ausdrücklich die eigenen Belange als nicht berührt betrachtete. Gut ein Drittel der 43 Beteiligungsfälle $(15=34,9 \%$ von 43$)$ beim GAA-N sind ohne abfallrechtliche Auflagen geblieben. ${ }^{127}$ Auf der anderen Seite verbleibt eine Mehrheit von immerhin zwei Dritteln, in denen einschlägige Auflagen erteilt wurden. ${ }^{128}$ Man könnte aus diesem Befund den (vorsichtigen) Schluß ziehen, daß eine beteiligte Behörde die eingeräumte Gelegenheit, ihre Interessen wahrzunehmen, auch soweit wie möglich ausschöpft. Die Frage, wieweit die in den beiden Genehmigungsmodellen unterschied-

126 LRA-2 nur zu 41,7\%.

127 S. hierzu unten Ziff. 3.3.1 mit Tab. 30. Ein vergleichbarer Anteil an »Fehlanzeigen« hinsichtlich eigener Belange findet sich bei keiner der übrigen beteiligten Fachbehörden.

128 Ein (statistischer) Vergleich mit dem LRA-1 ist nicht möglich, da dort nur in drei Verfahren eine förmliche Beteiligung der Abfallbehörde erfolgt war, die in einem Fall zu Auflagen führte. Beim LRA-2, das im Hinblick auf die abfallrechtlichen Belange ebenfalls eine eher restriktive Beteiligungspraxis vollzog, belaufen sich die einschlägigen Verfahren auf acht Fälle, von denen in sieben auch Auflagen ergingen. 
liche Beteiligung der Abfallbehörden im Einzelfall sich auch »sachlich rechtfertigt $\ll$, muß offenbleiben. ${ }^{129}$ Jedenfalls ist eine vergleichbar unterschiedliche Selektivität der Beteiligungspraxis bei den anderen, in der Tabelle namentlich aufgeführten Fachbehörden nicht zu beobachten. Die Besonderheiten im Umgang mit den abfallrechtlichen bzw. abfallwirtschaftlichen Belangen könnte sich zumindest zum Teil aus unterschiedlichen Abfallwirtschaftskonzepten und -regelungen der niedersächsischen bzw. baden-württembergischen Kommunen erklären, die in diesem Bereich über eine größere Autonomie verfügen als bei den von den anderen Fachbehörden »verwalteten « öffentlich-rechtlichen Belangen.

Das im Nord-Süd-Vergleich anteilige Ungleichgewicht in der Beteiligung der Abfallbehörde findet ein (gleichsam seitenverkehrtes) Pendant in der Beteiligung der Kommunen. ${ }^{130}$ Deren mögliche Betroffenheit resultiert aus ihrer Eigenschaft als (bau-)planungsrechtlicher Hoheitsträger für den Standort, auf dem sich das zur Genehmigung anstehende Vorhaben entweder bereits befindet oder der dafür vorgesehen ist. ${ }^{131}$ Während das badenwürttembergische LRA die Kommunen fast ausnahmslos anhörte, ${ }^{132}$ geschah dies beim niedersächsischen GAA-N nur in einem Drittel aller Verfahren $(32,8 \%)$. Darin sind zudem sechs Fälle (10,3\%) enthalten, in denen sich die Anhörung nicht der Initiative des GAA-N verdankte, sondern der des beteiligten Ordnungsamts des Landkreises, dem die betroffene Gemeinde angehörte. ${ }^{133}$ Die unterschiedliche Beteiligungspraxis bei den Kommunen scheint - wie auch bei den Abfallbehörden - nicht in den modellbedingten organisatorischen Unterschieden zu gründen, sondern eher in verschiedenen kommunalpolitischen Traditionen. Der im Süden vom Kreistag gewählte Landrat dürfte infolge der Doppelfunktion des Landkreises - einerseits als Selbstverwaltungskörperschaft Träger von öffentlichen Aufgaben im eigenen Wirkungskreis, andererseits als (untere) Verwaltungsbehörde Träger von Aufgaben im übertragenen Wirkungskreis -

129 Grundsätzlich läßt sich aus der Nichtbeteiligung einer Fachbehörde nicht ohne weiteres auf die (subjektive oder auch »objektive«) Nichtbetroffenheit ihrer Belange schließen.

130 Die im Nord-Süd-Vergleich beim GAA-N höheren und beim LRA-1 niedrigeren Beteiligungsanteile bei den Abfallbehörden werden durch die gegenläufigen, beim GAA-N niedrigeren und beim LRA-1 höheren Beteiligungsanteile bei den Kommunen nahezu vollständig kompensiert, so daß sie sich auf die durchschnittliche Anzahl der je Verfahren beteiligten Behörden und Ämter in beiden Organisationsmodellen (vgl. oben Ziff. 2.3.1 mit Tab. 13.3) nicht auswirken.

131 Die im Einzelfall ggf. erforderliche planungsrechtliche Zustimmung der Kommune ergibt sich aus der gemäß dem Konzentrationsprinzip ( $\$ 13$ BImSchG) mit zu erteilenden Baugenehmigung.

132 Beim LRA-1 hat es lediglich in zwei, beim LRA-2 in nur einem einzigen Verfahren keine Beteiligung gegeben.

133 Der Unterschied ist hochsignifikant, selbst wenn man die nicht durch das GAA-N veranlaßten Beteiligungsfälle mit einbezieht $\left(\mathrm{chi}^{2}=43,35>10,82, \mathrm{p}=0,001, \mathrm{df}=1\right)$. 
»kommunalen« Interessen im Landkreis größere Aufmerksamkeit zu schenken geneigt sein als das niedersächsische GAA-N mit seinem Status als zentrale, von der kommunalen Ebene unabhängige staatliche Genehmigungsbehörde. Offenbar begünstigt die organisatorische Einbindung der süddeutschen Genehmigungsbehörde in die Kreisverwaltung einen eher kooperativen und konfliktvermeidenden Umgang mit den Gemeinden, zumindest läßt die auf Grund der Kommunalverfassung politisch relativ starke Stellung der Gemeinden einen solchen Umgang angeraten erscheinen. ${ }^{134}$ Dafür spricht nicht zuletzt, daß selbst in den Fällen, in denen planungsrechtliche kommunale Belange von vornherein nicht betroffen sein können, gleichwohl die Gemeinde angehört wird. ${ }^{135}$ Ergebnis dieser Praxis ist der schon erwähnte Befund eines nahezu 100\%igen Beteiligungsanteils der Kommunen. Die mutmaßlich in erster Linie verwaltungspolitisch und nicht aus der Sache begründete Beteiligung spiegelt sich auch in der im Ergebnis geringen planungsrechtlich fundierten Einflußnahme der Gemeinden auf den Genehmigungsinhalt wider. Darauf gestützte Auflagen oder Beschränkungen für Errichtung oder Betrieb der beantragten Anlage bzw. Anlagenänderung finden sich beim LRA-1 lediglich in einer Minderzahl der Genehmigungsbescheide $(11,6 \%)$, ein Anteil, der nur geringfügig den entsprechenden Anteil beim GAA-N mit 6,9\% übersteigt (vgl. dazu auch unten Ziff. 3.3.1). ${ }^{136}$

Sieht man vom Abfallbereich und den Kommunen ab, geht mit der unterschiedlichen Organisation der Genehmigungszuständigkeit im Ergebnis keine unterschiedliche Praxis im Hinblick auf Art und Umfang der Beteiligung externer Fachbehörden einher. Von daher muß der Antragsteller im Norden wie im Süden in vergleichbarem Maße damit rechnen, mit (von der Genehmigungsbehörde praktisch als verbindlich behandelten) Auflagen auch anderer Fachbehörden beschwert zu werden. Der organisationsbedingt größere Entscheidungsspielraum, über den das baden-württembergische LRA gegenüber dem niedersächsischen GAA-N bei der Auswahl der Fachbehörden faktisch verfügt, bleibt insoweit folgenlos. Unter dem Ge-

$134 \mathrm{Vgl}$. oben Anm. 43.

135 Damit kann sich die Genehmigungsbehörde freilich insofern zusätzliche Probleme einhandeln, als die Gemeinden vielfach geneigt sind, bei Gelegenheit ihrer Beteiligung von ihren planungsrechtlichen Hoheitsrechten nicht gedeckte Ziele zu verfolgen. Das kann für den Betreiber u.U. zusätzliche Einschränkungen für Errichtung oder Betrieb der Anlage mit sich bringen (vgl. hierzu auch Harder 1987, S. $31 \mathrm{ff}$.). In den hier betrachteten vereinfachten Verfahren kam es allerdings insgesamt nur in einer Minderzahl (13) zu Konflikten mit den Gemeinden, wobei tatsächlich vergleichsweise mehr Fälle auf den großzügig Beteiligung gewährenden Süden (LRA1: 7; LRA-2: 3) als auf den in dieser Hinsicht deutlich restriktiver vorgehenden Norden (GAA-N: 3) entfallen. Vgl. hierzu auch unten Ziff. 3.1.3.

136 Beim LRA-2 liegt der entsprechende Anteil sogar bei nur 4,2\% (= ein einziges Verfahren) und damit noch unter dem des GAA-N. 
sichtspunkt der Arbeitsbelastung befindet sich allerdings das GAA-N in der günstigeren Position. Das vom LRA selbst zu organisierende Einholen und ggf. erforderliche Anmahnen der einzelnen Stellungnahmen delegiert das GAA-N weitgehend an die jeweils zuständige »Zentrale « der allgemeinen Verwaltung des Landkreises bzw. der (kreisfreien) Städte. ${ }^{137}$ Hinzu kommt, daß das baden-württembergische LRA modellbedingt mit dem GAA stets eine Behörde mehr als das niedersächsische GAA-N hinzuzuziehen hat. Tatsächlich nahm die Phase der Fachbehördenbeiziehung im Durchschnitt beim LRA-1 (und auch beim LRA-2) erheblich mehr Zeit in Anspruch als beim GAA-N und beeinflußte insoweit auch die Gesamtdauer der Genehmigungsverfahren. ${ }^{138}$

\subsection{Kommunikation zwischen Antragsteller und Genehmigungsbehörde}

\subsubsection{Häufigkeit der Kontakte (Kommunikationsdichte)}

Einen Überblick über die Häufigkeit unmittelbarer (dokumentierter) Kontakte, die zwischen Antragsteller und Genehmigungsbehörde nach Antragseingang bis zur Überstellung des Genehmigungsbescheids stattgefunden haben, gibt Tab. 15. Dabei ist im Süden - wie auch schon bei den Vorantragsberatungen (vgl. 1.3.1) - aus Vergleichsgründen das zugehörige GAA mit einbezogen.

137 Von daher stellte sich für das GAA-N in den Fällen, in denen es nur bei der Kommunalverwaltung angesiedelte Behörden in die Beteiligung einbezog, erst gar nicht das Problem der mit Blick auf eine Verfahrensbeschleunigung in $\S 11$ Satz 2 der 9. BImSchV vorgeschriebenen »sternförmigen«, als zeitgleich zu verstehenden Behördenbeteiligung. Das betraf allein die Hälfte $(50,0 \%)$ aller Verfahren mit Behördenbeteiligung. In den übrigen Fällen, in denen nicht zur Kommunalverwaltung gehörende Fachämter gehört wurden, erfolgte die Beteiligung in drei Vierteln dieser Teilmenge $(75,0 \%)$ »sternförmig «, was bezogen auf alle Verfahren mit Behördenbeiziehung einen Anteil von 37,5\% ausmacht. Beim LRA-1, dem in jedem Fall die Koordination sämtlicher anzuhörenden Fachbehörden obliegt, wurde die Vorschrift dagegen häufiger, nämlich bei $44,2 \%$ aller Verfahren erfüllt, beim insoweit vergleichbaren LRA-2 allerdings lediglich in einem Viertel (25,0\%). Vgl. hierzu auch unten Ziff. 4.3.3.1 mit Tab. 41.2 (erste Kategorie).

$138 \mathrm{Vgl}$. hierzu ausführlicher unten Ziff. 4.3.3.1 mit Tab. 41.21 und Ziff, 4.4 mit Tab. 46 und Tab. 46.1 . 
Tab. 15

Anzahl der (dokumentierten) Kontakte zwischen Antragsteller und Genehmigungsbehörde* nach Antragseingang (Häufigkeitsverteilung)

\begin{tabular}{|l|c|c|c|}
\cline { 2 - 4 } \multicolumn{1}{c|}{} & $G A A-N$ & $L R A-1$ & $L R A-2$ \\
\hline Keiner & 14 & 6 & 5 \\
& $24,1 \%$ & $14,0 \%$ & $20,8 \%$ \\
\hline \multirow{2}{*}{ Einer } & 13 & 7 & 5 \\
& $22,4 \%$ & $16,3 \%$ & $20,8 \%$ \\
\hline Zwei & 9 & 8 & 5 \\
Drei bis vier & $15,5 \%$ & $18,6 \%$ & $20,8 \%$ \\
\hline Fünf bis sechs & 11 & 6 & 4 \\
\hline Sieben und mehr** & $19,0 \%$ & $14,0 \%$ & $16,7 \%$ \\
\hline \hline Verfahren gesamt*** & 4 & 4 & 4 \\
\hline
\end{tabular}

* Aus Vergleichsgründen im Süden LRA und zugehöriges GAA.

** Maximale Kontaktzahl beim GAA-N: 20, LRA-1: 21, LRA-2: 7.

*** Abweichungen von 100,0\% durch Rundungen bei den prozentualen Einzelhäufigkeiten.

Wie die Befunde zeigen, stellen Verfahren, die in diesem Zeitraum (nach Aktenlage) ohne jeglichen direkten Kontakt zwischen den Hauptbeteiligten verlaufen sind (Häufigkeitsklasse: »Keiner «), nur eine Minderheit dar. Der entsprechende Anteil fällt beim GAA-N mit einem knappen Viertel $(24,1 \%)$ zwar höher aus als beim LRA-1 mit rd. einem Siebtel $(14,0 \%)$, liegt aber unterhalb statistischer Signifikanz. ${ }^{139}$

In der Mehrheit der Genehmigungsverfahren kam es mithin zu mindestens einem direkten (dokumentierten) Kontakt. Dabei ergeben sich in den einzelnen, (nach Anzahl der je Verfahren erfolgten Interaktionen) aufsteigenden Häufigkeitsklassen bei beiden Genehmigungsbehörden weitgehend ähnliche Anteile. Davon auszunehmen ist allerdings die letzte Häufigkeitsklasse mit sieben und mehr Kontakten. Hier beläuft sich der Anteil beim LRA-1 mit 27,9\% gegenüber dem beim GAA-N von lediglich $12,1 \%$ auf mehr als das Doppelte, wobei die beiden Behörden im Hinblick auf die maximale Kontaktzahl in ein und demselben Verfahren mit 21 bzw. 20 Kon- 
takten nahezu gleichauf liegen. ${ }^{140}$ Hauptsächlich diese (signifikante) ${ }^{141} \mathrm{Di}$ vergenz sorgt im Verbund mit der gleichgerichteten, wenn auch für sich genommen nicht signifikanten Differenz in der untersten Häufigkeitsklasse für einen signifikant höheren Wert in der durchschnittlichen Interaktionszahl pro Verfahren von 4,4 Kontakten beim LRA-1 gegenüber 2,8 Kontakten beim GAA-N. ${ }^{142}$

Die schon bei der Vorantragsberatung beobachtete höhere Interaktionsdichte zwischen Antragsteller und Genehmigungsbehörde beim LRA-1 im Vergleich zum GAA-N (vgl. oben Ziff. 1.3.1, Tab. 8) setzt sich demnach in noch deutlicherer Ausprägung - auch im nachfolgenden »offiziellen « Verfahrensgang fort. Dies gilt jedenfalls für den Durchschnitt der Verfahren. Die Frage ist, wieweit dies auch auf das einzelne Verfahren zutrifft. Sie zielt darauf, ob Verfahren, die sich im Vorantragszeitraum als beratungsintensiv erwiesen haben, auch während der Antragsbearbeitung mit einer überdurchschnittlichen Kommunikationsdichte zwischen den Hauptbeteiligten einhergehen oder ob Beratungen in der Vorantragszeit den Kommunikationsbedarf im weiteren Verfahrensgang (i.S. einer Substitution) senken.

Tab. 16.1 gibt zunächst einen Überblick über die Kontakthäufigkeit während des gesamten Verfahrensverlaufs vom Vorantragszeitraum bis zur Genehmigungserteilung. Anschaulicher treten die gegenläufigen Tendenzen zwischen GAA-N und LRA-1 hervor, wenn man die Verteilung auf vier Häufigkeitsklassen verdichtet (Tab. 16.2). Läßt man einmal die wenigen Verfahren ohne dokumentierte Kommunikation außen vor, entfallen auf die übrigen drei Häufigkeitsklassen beim GAA-N mit steigender Kontaktfrequenz abnehmende Anteile (von 55,2\% über 24,1\% bis $13,8 \%$ ), während die Anteile beim LRA-1 zunehmen (von $27,9 \%$ über $30,2 \%$ bis $39,5 \%$ ). Oder anders gesagt: Beim GAA-N kam es in rd. $62 \%$ der Verfahren zu höchstens fünf Kontakten und in $38 \%$ zu mehr als fünf Kontakten, beim LRA-1 dagegen nur in rd. 30\% zu höchstens fünf Kontakten und in $70 \% \mathrm{zu}$ mehr als fünf Kontakten. Die durchschnittliche Kontaktzahl pro Verfahren

140 Das LRA-2 hebt sich hier mit einem Anteil von nur 4,2\% deutlich von den beiden anderen Ämtern ab. Das gilt auch für die lediglich bei sieben Kontakten liegende maximale Kontaktzahl.

$141 \mathrm{chi}^{2}=4,05>3,84, \mathrm{p}=0,05, \mathrm{df}=1$.

$142 \mathrm{t}=2,02>\mathrm{t}_{(0,975 ; 99)}=1,984$. Die Differenz der Varianzen (GAA-N: 12,59 Mon., LRA-1: 20,54 Mon.) ist nicht signifikant $\left(F=1,63<\mathrm{F}_{(0,975 ; 42,57)}=1,75\right)$. - Beim LRA-2 ist die Kontaktdichte mit durchschnittlich 2,4 am geringsten bei einer signifikant niedrigeren Varianz (4,32 Mon.) gegenüber den beiden anderen Ämtern (in bezug auf GAA-N: $\mathrm{F}=2,91>\mathrm{F}_{(0,995 ; 57,23)}=2,77$, in bezug auf LRA-1: $\mathrm{F}=4,698$ $\left.>\mathrm{F}_{(0,999 ; 42,23)}=3,77\right)$. 
beim LRA-1 mit 8,9 Kontakten übersteigt signifikant die beim GAA-N mit 5,7 Kontakten. ${ }^{143}$

Tab. 16.1

Anzahl der (dokumentierten) Kontakte zwischen Antragsteller und Genehmigungsbehörde* ab Vorantragszeitraum bis Genehmigungsbescheid (Häufigkeitsverteilung)

\begin{tabular}{|l|c|c|c|}
\cline { 2 - 4 } \multicolumn{1}{c|}{} & GAA-N & LRA-1 & LRA-2 \\
\hline Keiner & 4 & 1 & 1 \\
\hline \multirow{2}{*}{ Einer } & $6,9 \%$ & $2,3 \%$ & $4,2 \%$ \\
\hline Zwei & 2 & 2 & 1 \\
& $3,4 \%$ & $4,7 \%$ & $4,2 \%$ \\
\hline Drei bis vier & 7 & 0 & 6 \\
& $12,1 \%$ & $0,0 \%$ & $25,0 \%$ \\
\hline Fünf bis sechs & 15 & 9 & 6 \\
\hline \multirow{2}{*}{ Sieben bis zehn } & $25,9 \%$ & $20,9 \%$ & $25,0 \%$ \\
\hline Mehr als zehn** & 12 & 4 & 3 \\
\hline \hline Verfahren gesamt*** & $20,7 \%$ & $9,3 \%$ & $12,5 \%$ \\
\hline \hline
\end{tabular}

* Aus Vergleichsgründen im Süden LRA und zugehöriges GAA.

** Maximale Kontaktzahl beim GAA-N: 25, LRA-1: 25, LRA-2: 14.

*** Abweichungen von 100,0\% durch Rundungen bei den prozentualen Einzelhäufigkeiten.

$143 \mathrm{t}=3,075>\mathrm{t}_{(0,995 ; 99)}=2,626$ bei statistisch nicht differierenden Varianzen (GAAN: 22,16 Mon., LRA-1: 29,85 Mon.; $\left.F=1,35<\mathrm{F}_{(0,90 ; 42,57)}=1,41\right)$. - Beim LRA2 ist die Tendenz der Verteilung zugunsten niedrigerer Kontaktfrequenzen noch etwas stärker ausgeprägt als beim GAA-N, was sich auch in einem etwas niedrigeren Mittelwert von 5,1 spiegelt. 
Tab. 16.2

Anzahl der (dokumentierten) Kontakte zwischen Antragsteller und Genehmigungsbehörde* ab Vorantragszeitraum bis Genehmigungsbescheid (komprimierte Häufigkeitsverteilung)

\begin{tabular}{|l|c|c|c|}
\cline { 2 - 4 } \multicolumn{1}{c|}{} & GAA-N & LRA-1 & LRA-2 \\
\hline \multirow{2}{*}{ Keiner } & 4 & 1 & 1 \\
& $6,9 \%$ & $2,3 \%$ & $4,2 \%$ \\
\hline \multirow{2}{*}{ Einer bis fünf } & 32 & 12 & 15 \\
& $55,2 \%$ & $27,9 \%$ & $62,5 \%$ \\
\hline \multirow{2}{*}{ Sechs bis zehn } & 14 & 13 & 5 \\
\hline \multirow{2}{*}{ Mehr als zehn** } & $24,1 \%$ & $30,2 \%$ & $20,8 \%$ \\
\hline \hline \multirow{2}{*}{ Verfahren gesamt*** } & 8 & 17 & 3 \\
\hline \hline
\end{tabular}

* Aus Vergleichsgründen im Süden LRA und zugehöriges GAA.

** Maximale Kontaktzahl beim GAA-N: 25, LRA-1: 25, LRA-2: 14.

*** Abweichungen von $100,0 \%$ durch Rundungen bei den prozentualen Einzelhäufigkeiten.

Mit Blick auf die aufgeworfene Frage, wieweit Vorantragsberatungen den (antragsbezogenen) Kommunikationsbedarf zwischen Genehmigungsbehörde und Antragsteller ausschöpfen und insoweit die Phase der Antragsbearbeitung entlasten, unterteilt Tab. 16.3 die stattgefundenen Kontakte nach den beiden Verfahrensphasen vor und nach (offizieller) Antragstellung. Danach liegt der Anteil an Verfahren, in denen sich die Kommunikation auf den Vorantragszeitraum beschränkte, beim GAA-N etwas über dem beim LRA-1 (20,7\% gegenüber 11,6\%). Auch die Anteile der Verfahren, in denen die Kontaktaufnahme erst nach Antragstellung erfolgte, bewegen sich bei beiden Behörden auf ähnlichem (niedrigen) Niveau (10,3\% bzw. 7,0\%). In der großen Mehrheit der Verfahren erstreckte sich somit die Kommunikation auf beide Verfahrensphasen, beim LRA-1 mit 79,1\% allerdings zu einem (aber nicht signifikant) höheren Anteil als beim GAA-N mit $62,1 \% .^{144}$

$144 \mathrm{chi}^{2}=2,16<2,71$ für $\mathrm{p}=0,1$ und $\mathrm{df}=1$. 
Tab. 16.3

Anzahl der Verfahren mit (kumulierten) Kontakten zwischen Antragsteller und Genehmigungsbehörde* im Vorantragszeitraum und während der Antragsbearbeitung

\begin{tabular}{|l|c|c|c|}
\cline { 2 - 4 } \multicolumn{1}{c|}{} & GAA-N & LRA-1 & LRA-2 \\
\hline Verfahren ohne Kontakte & 4 & 1 & 1 \\
in beiden Verfahrensphasen & $6,9 \%$ & $2,3 \%$ & $4,2 \%$ \\
\hline Verfahren mit Kontakten & 12 & 5 & 4 \\
nur im Vorantragszeitraum & $20,7 \%$ & $11,6 \%$ & $16,7 \%$ \\
\hline Verfahren mit Kontakten & 6 & 3 & 4 \\
nur nach Antragstellung & $10,3 \%$ & $7,0 \%$ & $16,7 \%$ \\
\hline Verfahren mit Kontakten & 36 & 34 & 15 \\
in beiden Verfahrensphasen & $62,1 \%$ & $79,1 \%$ & $62,5 \%$ \\
\hline \hline Verfahren gesamt*** & 58 & 43 & 24 \\
\hline
\end{tabular}

* Aus Vergleichsgründen im Süden LRA und zugehöriges GAA.

** Abweichungen von 100,0\% durch Rundungen bei den prozentualen Einzelhäufigkeiten.

Zusammengefaßt erlauben die Befunde zwei Schlußfolgerungen. Zum einen zeigen sie, daß Vorantragsberatungen in beiden Genehmigungsmodellen nur zu einem kleineren Teil weitere Kommunikation während der Antragsbearbeitung zwischen den Hauptbeteiligten erübrigen. Dies läßt darauf schließen, daß sich - modellunabhängig - aus behördlicher Sicht »schwierige« Fälle bereits in einem frühen Stadium des Verfahrens herauskristallisieren und dann auch weiterhin als solche behandelt werden. Zum zweiten zeigen die Daten, daß die Genehmigungsverfahren im Süden in beiden Phasen - vor und nach Antragstellung - in größerem Umfang von direkter Kommunikation zwischen Genehmigungsbehörde und Antragsteller begleitet sind als im Norden. Dies könnte auch daran liegen, daß die süddeutsche Genehmigungsbehörde den Antragsteller in den Fällen, in denen sie sich durch dessen fachtechnisches Auskunfts- bzw. Beratungsersuchen überfordert sieht, an das zugehörige GAA weiterverweist bzw. weiterverweisen muß. Derartige, die Kontaktfrequenz erhöhende Konstellationen sind bei der norddeutschen, auch fachlich zuständigen Genehmigungsbehörde ausgeschlossen. 
2.4.2. Direkte und indirekte Beteiligung von Fachbehörden an der Kommunikation zwischen Antragsteller und Genehmigungsbehörde

Für die These, daß die beim LRA-1 im Vergleich zum GAA-N höhere Kontaktdichte zwischen Genehmigungsbehörde und Antragsteller im Verlauf der Antragsbearbeitung trotz vorausgegangener höherer Beratungstätigkeit im Vorantragszeitraum zumindest zum Teil, wenn nicht in erster Linie auf die unterschiedlich organisierte Genehmigungskompetenz zurückzuführen ist, spricht ein weiterer Befund. Er betrifft das Ausmaß direkter bzw. indirekter Beteiligung von Fachbehörden an der Kommunikation zwischen Antragsteller und Genehmigungsbehörde.

Tab. 17

Fachbehördenbeteiligung bei den Kontakten zwischen Genehmigungsbehörde und Antragsteller in der Zeit der Antragsbearbeitung

\begin{tabular}{|c|c|c|c|}
\hline & \\
\hline & $G A A-N$ & $L R A-1$ & $L R A-2$ \\
\hline $\begin{array}{l}\text { a) Ohne Beteiligung sonstiger Fachbehör- } \\
\text { den* }^{*}\end{array}$ & $\begin{array}{c}21 \\
36,2 \%\end{array}$ & $\begin{array}{c}17 \\
39,5 \%\end{array}$ & $\begin{array}{c}11 \\
45,8 \%\end{array}$ \\
\hline $\begin{array}{l}\text { b) Direkte Beteiligung sonstiger Fachbe- } \\
\text { hörden* } \\
\text { [darunter: sog. Antragskonferenzen] }\end{array}$ & $\begin{array}{c}14 \\
24,1 \% \\
{[5]}\end{array}$ & $\begin{array}{c}4 \\
9,3 \% \\
{[1]}\end{array}$ & $\begin{array}{c}1 \\
4,2 \% \\
{[1]}\end{array}$ \\
\hline $\begin{array}{l}\text { c) Nur indirekte Beteiligung sonstiger } \\
\text { Fachbehörden* }\end{array}$ & $\begin{array}{c}9 \\
15,5 \%\end{array}$ & $\begin{array}{c}16 \\
37,2 \%\end{array}$ & $\begin{array}{c}7 \\
29,2 \%\end{array}$ \\
\hline $\begin{array}{l}\text { [a)-c): mit direkter und/oder indirekter } \\
\text { Beteiligung von GAA-1 bzw. GAA-2] }\end{array}$ & $\begin{array}{l}{[-]} \\
{[-]}\end{array}$ & $\begin{array}{c}{[26]} \\
{[60,5 \%]}\end{array}$ & $\begin{array}{c}{[10]} \\
{[41,7 \%]}\end{array}$ \\
\hline Verfahren ohne dokumentierte Kontakte & $\begin{array}{c}14 \\
24,1 \%\end{array}$ & $\begin{array}{c}6 \\
14,0 \%\end{array}$ & $\begin{array}{c}5 \\
20,8 \%\end{array}$ \\
\hline Verfahren gesamt** & $\begin{array}{c}58 \\
99,9 \%\end{array}$ & $\begin{array}{c}43 \\
100,0 \%\end{array}$ & $\begin{array}{c}24 \\
100,0 \%\end{array}$ \\
\hline
\end{tabular}

* Aus Vergleichsgründen im Süden mit zugehörigem GAA.

** Abweichungen von 100,0\% durch Rundungen bei den prozentualen (nur kursiv gekennzeichneten) Einzelhäufigkeiten.

Tab. 17 schlüsselt dazu die Verfahren nach folgenden Beteiligtenkonstellationen auf: Verfahren, in denen sich die Kontakte ausschließlich zwischen Antragsteller und Genehmigungsbehörde (im Süden aus Vergleichsgründen einschließlich des zugehörigen GAA) abspielen (Kategorie a der Tabelle), 
Verfahren, in denen mindestens ein Kontakt unter direkter Teilnahme i.S. persönlicher Anwesenheit (mindestens) eines Mitarbeiters einer oder mehrerer (sonstiger) Fachbehörden stattfindet (Kategorie b), und schließlich Verfahren, in denen (sonstige) Fachbehörden lediglich indirekt durch Übermittlung von Antragsnachforderungen und fachspezifischen Erfordernissen an den Antragsteller über die Genehmigungsbehörde beteiligt sind (Kategorie c). Zusätzlich (kleingedruckte Kategorie [a - c]) ist für den Süden die direkte oder indirekte Mitwirkung des zugehörigen GAA-1 bzw. GAA-2 innerhalb einer der drei fachbehördlichen Beteiligungskonstellationen ausgewiesen. ${ }^{145}$

Wie aus der Tabelle hervorgeht, beschränkten sich beim GAA-N und beim LRA-1 in knapp bzw. rund zwei Fünfteln der Verfahren (36,2\% bzw. $39,5 \%$ ) die Kontakte mit dem Antragsteller auf die Genehmigungsbehörden bzw. im Süden einschließlich des zugehörigen GAA (Kategorie a). Etwa ähnlich hoch fallen mit 39,6\% beim GAA-N und 46,5\% beim LRA-1 auch die Anteile der Verfahren aus, in denen (sonstige) Fachbehörden in direkter Form oder indirekter Form in die Kommunikation einbezogen wurden bzw. sich selbst einschalteten (Kategorien b plus c). Während beim GAA-N aber die direkte $(24,1 \%)$ gegenüber einer lediglich indirekten $(15,5 \%)$ Beteiligung überwiegt, verhält es sich beim LRA-1 mit einem deutlichen Überwiegen indirekter $(37,2 \%)$ gegenüber direkter $(9,3 \%)$ Beteiligung umge-

145 Der Klarstellung halber sei ausdrücklich betont, daß es hier ausschließlich um direkte oder indirekte Kontakte der Behörden mit dem Antragsteller geht. Entsprechend nicht einbezogen sind sich ausschließlich behördenintern abspielende Kontakte, d.h. solche, über deren Ergebnis der Antragsteller nicht informiert wird und die insofern nicht Gegenstand weiterer Kommunikation werden können. Darunter fallen insbesondere die von den Fachbehörden an die Genehmigungsbehörde adressierten Stellungnahmen oder auch (bei Verzug) deren Anmahnung seitens der Genehmigungsbehörde, speziell im Süden zusätzlich interne Kontakte zwischen dem LRA und dem zugehörigen GAA zum Zwecke des Informationsaustauschs, der Abstimmung des Vorgehens gegenüber dem Antragsteller u.ä. Im übrigen kann zwar auf Grund der hier gewählten Erhebungsgrundlage nicht ausgeschlossen werden, daß zwischen Antragsteller und Fachbehörde (u.U. auch für den Entscheidungsverlauf maßgebliche) Kontakte gezielt »an der Genehmigungsbehörde vorbei« stattgefunden haben. Die Wahrscheinlichkeit dafür, daß derartige Kontakte in der Akte spurlos bleiben, wird aber als gering eingeschätzt. Denn die Fachbehörden können nicht auch »an der Genehmigungsbehörde vorbei « entscheiden, da sie auf Grund der rechtlichen Verfahrensstruktur ihre Position dieser gegenüber offenlegen müssen und von daher einer gewissen Fremdkontrolle unterliegen. Vom Ansatz her anders liegen die Dinge, wenn in Absprachen zwischen Fachbehörde und Antragsteller die Genehmigungsbehörde mit einbezogen wird oder bilaterale Absprachen zwischen Antragsteller und Genehmigungsbehörde getroffen werden. Aber auch hier sind der Informalität insofern Grenzen gesetzt, als das ausgehandelte Ergebnis am Ende in Gestalt des Genehmigungsbescheids rechtsförmlich verbindlich gemacht werden muß. Vgl. hierzu auch die übernächste Anm. 
kehrt. ${ }^{146}$ Außerdem wurde beim GAA-N in fünf Verfahren eine der oben (Ziff. 1.3.2) erwähnten Vorantragskonferenz vergleichbare sog. Antragskonferenz abgehalten, also eine frühzeitig nach offizieller Antragstellung anberaumte gemeinsame Besprechung mit in ihren Belangen betroffenen Fachbehörden vor bzw. im Rahmen der Eröffnung der förmlichen Behördenbeteiligung. Beim LRA-1 (wie auch beim LRA-2) trifft das lediglich jeweils in einem Fall zu (Zeile im Kleindruck in Kategorie b).

Die gegenüber dem GAA-N beim LRA-1 modellbedingt in Form des zugehörigen GAA separierte immissionsschutzbezogene Fachkompetenz wurde in der Phase der Antragsbearbeitung in drei Fünfteln (60,5\%) aller Verfahren direkt oder indirekt, d.h. vorschriftsmäßig über die Genehmigungsbehörde vermittelt, ${ }^{147}$ in die Kommunikation mit dem Antragsteller einbezogen (kleingedruckte Kategorie [a-c]). ${ }^{148}$ Das GAA-1 ist damit im Vergleich zu den in 46,5\% der Verfahren mitwirkenden sonstigen Fachbehörden das am häufigsten beteiligte Fachamt, was auch zu vermuten war. Eher hätte man in Anbetracht seiner zentralen fachlichen Relevanz einen noch höheren Anteil erwarten können. ${ }^{149} \mathrm{Daß}$ das nicht der Fall ist, deutet darauf hin, daß das LRA-1 bestrebt ist, so weit wie möglich das Verfahren in der eigenen Hand zu behalten. Dafür spricht auch der Befund, daß sich die Mitwirkung des GAA-1 - anders als bei den sonstigen Fachbehörden überwiegend im direkten, zudem vielfach auch von ihm selbst initiierten Kontakt mit dem Antragsteller vollzog. Nur bei einem knappen Drittel der Fälle (entsprechend 12,1\% aller Verfahren) beschränkte sich das GAA-1

146 Die Differenzen in den Anteilen direkter bzw. indirekter Fachbehördenbeteiligung sind signifikant: chi $^{2}=3,78>2,71, p=0,1$ bzw. chi $^{2}=6,35>5,02, p=0,025$, jeweils $\mathrm{df}=1$.

147 Der im Süden formell gebotene »Umweg « über den Dienstweg, den auch die sonstigen Fachbehörden bei Nachforderungen an den Antragsteller einzuhalten haben, soll die jederzeitige Kontrolle der Genehmigungsbehörde auch über den Verfahrensgang sicherstellen. Die Kontrollfunktion dürfte dabei in der Praxis weniger im Sinne einer inhaltlichen Prüfung fachbehördlicher (Nach-) Forderungen im Hinblick auf deren sachliche Berechtigung ausgeübt werden - dazu fehlt es der Genehmigungsbehörde i.d.R. an einer entsprechenden, den Fachbehörden gleichwertigen oder gar überlegenen fachlichen Kompetenz. In erster Linie dient sie der reinen Information über fachbehördlich relevante Rahmenbedingungen bzw. Entscheidungsgrundlagen des Genehmigungsgegenstands.

148 Beim LRA-2 ergibt sich mit 41,7\% ein deutlich niedrigerer Beteiligungsanteil des zugehörigen GAA-2, die Differenz zum LRA-1 ist aber (auf Grund der geringen Fallzahl beim LRA-2) statistisch nicht signifikant $\left(\mathrm{chi}^{2}=2,20<2,71\right.$ für $\mathrm{p}=0,1$ und $\mathrm{df}=1$ ).

149 Zumal der Unterschied in den Beteiligungsanteilen zwischen dem GAA-1 und den sonstigen Fachbehörden unterhalb der statistischen Signifikanzgrenze bleibt $\left(\mathrm{chi}^{2}\right.$ $=1,68<2,71$ für $\mathrm{p}=0,1$ und $\mathrm{df}=1$ ). 
auf die Einhaltung des Dienstwegs. ${ }^{150}$ Die gegenüber Niedersachsen »ausgelagerte « immissionsschutzbezogene Fachkompetenz erhöht so in jedem Fall die Kommunikationsbelastung der baden-württembergischen Genehmigungsbehörde durch zusätzliche Kontakte mit dem Antragsteller. Außerdem kommen (hier nicht dargestellte) interne Kontakte zwischen diesen beiden Ämtern hinzu. ${ }^{151}$

Die aufgezeigten Unterschiede hinsichtlich der direkten Beteiligung von (sonstigen) Fachbehörden wie auch hinsichtlich der Durchführung von Antragskonferenzen während der Antragsbearbeitung relativieren sich allerdings, wenn man den Vorantragszeitraum hinzunimmt (Tab. 17.1). In dieser Phase waren beim LRA-1 bereits in 30,2\%, beim GAA-N aber lediglich in $12,1 \%$ aller Verfahren Fachbehörden bei der Beratung beteiligt worden, darunter beim LRA-1 in acht Fällen bzw. 18,6\% der Verfahren, beim GAA$\mathrm{N}$ in fünf Fällen bzw. 8,6\% der Verfahren auch in Form einer Vorantragskonferenz. ${ }^{152}$ Wie die Tabelle zeigt, wurden über beide Phasen der Genehmigungsverfahren hinweg im Ergebnis die Fachbehörden bei beiden Genehmigungsbehörden gleichermaßen beteiligt, beim GAA-N in 36,2\% der Fälle, beim LRA-1 in 39,5\% der Fälle (Kategorien b plus c plus d). ${ }^{153}$ Ähnliches gilt für die Durchführung einer Vorantrags- bzw. Antragskonferenz ${ }^{154}$ mit zehn Fällen bzw. 17,2\% aller Verfahren beim GAA-N und mit neun Fällen bzw. 20,9\% aller Verfahren beim LRA-1 (Zeilen im Kleindruck in Kategorie b bzw. c). ${ }^{155}$

Zusammengefaßt ergibt sich, daß in beiden Organisationsmodellen die (sonstigen) Fachbehörden in etwa gleichem Maße vor Abfassung ihrer Stellungnahmen in das Genehmigungsverfahren einbezogen werden, sei es, daß dies auf Initiative der Genehmigungsbehörde, sei es, daß dies auf eigene Initiative hin geschieht. Lediglich für den Zeitpunkt ihres Eintretens in das Verfahren zeigt sich eine unterschiedliche Gewichtung zugunsten der Vorantragsphase beim LRA-1. Eine andere Frage ist, ob die weitgehende Indifferenz der beiden Modelle in der formalen und kommunikativen Mitwirkung der Fachbehörden auch für deren inhaltlichen Einfluß auf die Genehmigungserteilung gilt (s. dazu unten Ziff. 3.3.1).

150 Beim GAA-2 verhält es sich umgekehrt. Hier überwiegt mit gut zwei Dritteln der Fälle (entsprechend 29,2\% aller Verfahren) der Dienstweg.

151 Vgl. hierzu oben Anm. 145.

152 Vgl. oben Ziff. 1.3.2, Tab. 9. Die dort berechneten Anteile sind hier umprozentuiert auf die jeweilige Grundgesamtheit der Genehmigungs verfahren. Vgl. auch die nachfolgende Tab. 17.1, Kategorien b bzw. d.

153 Beim LRA-2 fällt der Anteil mit 12,5\% aller Verfahren erheblich niedriger aus.

154 Eine Kumulation von Vorantrags- und Antragskonferenz in ein und demselben Verfahren kam dabei nicht vor.

155 Beim LRA-2 sind es mit lediglich zwei Fällen bzw. 8,3\% aller Verfahren deutlich weniger. 
Tab. 17.1

Direkte Fachbehördenbeteiligung* bei den Kontakten zwischen Genehmigungsbehörde und Antragsteller ab Vorantragszeitraum bis Genehmigungsbescheid

\begin{tabular}{|l|c|c|c|}
\cline { 2 - 4 } \multicolumn{1}{c|}{} & $G A A-N$ & $L R A-1$ & $L R A-2$ \\
\hline a) Ohne direkte Fachbehördenbeteiligung & 33 & 25 & 20 \\
& $56,9 \%$ & $58,1 \%$ & $83,3 \%$ \\
\hline $\begin{array}{l}\text { b) Direkte Fachbehördenbeteiligung } \\
\text { nur im Vorantragszeitraum } \\
\text { [darunter: sog. Vorantragskonferenzen] }\end{array}$ & 7 & 13 & 2 \\
\hline c) Direkte Fachbehördenbeteiligung nur & {$[5]$} & $30,2 \%$ & $8,3 \%$ \\
nach Antragstellung & 10 & {$[8]$} & {$[1]$} \\
\hline [darunter: sog. Vorantragskonferenzen] & $17,2 \%$ & $4,3 \%$ & $4,2 \%$ \\
\hline d) Direkte Fachbehördenbeteiligung in & {$[5]$} & {$[1]$} & {$[1]$} \\
\hline beiden Phasen & $6,9 \%$ & 0 & 0 \\
\hline Verfahren ohne Kontakte & 4 & 1 & $0,0 \%$ \\
\hline \hline Verfahren gesamt** & $6,9 \%$ & $2,3 \%$ & $4,2 \%$ \\
\hline
\end{tabular}

* Aus Vergleichsgründen im Süden mit zugehörigem GAA.

** Abweichungen von 100,0\% durch Rundungen bei den prozentualen Einzelhäufigkeiten.

Vor dem Hintergrund ähnlicher Teilhabe von Fachbehörden an der Erarbeitung der Entscheidungsgrundlagen dürfte die festgestellte höhere Kontakthäufigkeit nicht nur in der ersten, sondern noch ausgeprägter auch in der zweiten Verfahrensphase beim LRA-1 gegenüber dem GAA-N (vgl. oben Ziff. 2.4.1) in erster Linie auf die modellbedingte behördliche Trennung von formaler (immissionsschutzrechtlicher) Entscheidungs- und materieller Fachkompetenz im Süden zurückzuführen sein.

\subsubsection{Medium der Kommunikation zwischen Antragsteller und Genehmigungsbehörde}

Während die Vorantragsberatung in beiden Genehmigungsmodellen großteils auch persönliche Begegnungen zwischen Antragsteller und Genehmigungsbehörde eingeschlossen hat (vgl. oben Ziff. 1.3.3, Tab. 10), spielte sich der Hauptteil der Kommunikation zwischen den Hauptbeteiligten nach Antragseingang ausschließlich schriftlich ab (Brief und/oder in Einzelfäl- 
len auch Fax). Wie aus Tab. 18 hervorgeht, belaufen sich die entsprechenden Anteile auf 43,1\% beim GAA-N bzw. 58,1\% beim LRA-1. Etwa in der Hälfte dieser Fälle sind zusätzlich auch Telefonate (über Aktennotiz) belegt (Kategorie a). Dabei ist zu berücksichtigen, daß im Vergleich zur Vorantragsphase beim GAA-N wie auch beim LRA-1 jeweils bei einem größeren Teil der Verfahren von 24,1\% bzw. 14,0\% (gegenüber 17,2\% bzw. 9,3\%) keinerlei (weitere) Kontakte dokumentiert sind (Kategorie c). ${ }^{156}$

Es verbleibt ein beim GAA-N und LRA-1 jeweils etwa gleich hoher Anteil von jeweils rd. $30 \%$ der Verfahren (32,8\% bzw. 27,9\%), in denen persönliche Gespräche zwischen Antragsteller und Genehmigungsbehörde (im Süden unter Einschluß des zugehörigen GAA) stattgefunden haben (Kategorie b). ${ }^{157}$ Teils wurden die Gespräche im Amt geführt, ${ }^{158}$ teils in der Firma des Antragstellers (Kategorie b1 im Kleindruck), ${ }^{159}$ wobei es sich wie es bei den vergleichbaren Fällen in den Vorantragsberatungen schon zu registrieren war (vgl. oben Ziff. 1.3.3) - vorwiegend um größere Unternehmen handelt, die die Behördenvertreter in ihre Räumlichkeiten haben »bitten lassen«. Zur Klarstellung sei betont, daß die Kategorie der bei der Firma abgehaltenen Termine sich ausschließlich auf die Wahl des Gesprächsorts bezieht. Die Fälle, in denen die Behörde die Firma eigens zum Zwecke einer Betriebsbegehung, sei es i.S. einer lediglich informatorischen Besichtigung, sei es i.S. einer förmlichen Überprüfung der zur Genehmigung anstehenden Anlage aufsuchte, sind gesondert erfaßt (vgl. dazu nachfolgende Ziff. 2.4.5).

Was schon für die Vorantragsberatung festzustellen war, bestätigt sich auch für die Zeit der (»offiziellen«) Antragsbearbeitung: Die behördliche Dezentralisierung der Genehmigungszuständigkeit in Baden-Württemberg geht zwar mit einer höheren Kontaktdichte mit dem Antragsteller einher, die im Vergleich zu Niedersachsen geringere räumliche Distanz steigert aber nicht das Quantum an persönlichen Kontakten. Die Erklärung dürfte darin liegen, daß die dezentralisierte Genehmigungszuständigkeit durch eine (wie in Niedersachsen) zentralisierte immissionsschutzrechtliche Fachbehörde insofern konterkariert wird, als auf deren Mitwirkung das LRA mangels eigener Fachkompetenz kaum verzichten kann. Von daher dirigieren Bereitschaft und zeitliche Verfügbarkeit der Mitarbeiter des zugehörigen GAA die Häufigkeit der für die Entscheidungsfindung wichtigen

156 Der Differenz der auf Kategorie a) entfallenden Anteile zwischen GAA-N und LRA1 von 10,1 Prozentpunkten steht eine gegenläufige Differenz an Verfahren der auf Kategorie c) entfallenden Anteile von 15,0 Prozentpunkten gegenüber, die sich rechnerisch weitgehend ausgleichen.

157 Beim LRA-2 beläuft sich der Anteil auf nur 12,5\% der Verfahren.

158 Meist bei der Genehmigungsbehörde, in Einzelfällen beim GAA-N auch bei einer Fachbehörde und beim LRA-1 auch im GAA-1.

159 In jeweils zwei Fällen (beim GAA-N bzw. LRA-1) gab es Gespräche sowohl in der Firma als auch im Amt. 
Termine wie eben gemeinsame Besprechungen. In dieser Hinsicht unterscheidet sich das baden-württembergische GAA offenbar nicht von seinem niedersächsischen Pendant.

Tab. 18

Medium der Kommunikation zwischen Antragsteller und Genehmigungsbehörde* nach Antragseingang

\begin{tabular}{|l|c|c|c|}
\cline { 2 - 4 } \multicolumn{1}{c|}{} & GAA-N & LRA-1 & LRA-2 \\
\hline a) Nur schriftl./telefonisch & 25 & 25 & 16 \\
& $43,1 \%$ & $58,1 \%$ & $66,7 \%$ \\
\hline \multirow{2}{*}{ b) Auch persönlich } & 19 & 12 & 3 \\
& $32,8 \%$ & $27,9 \%$ & $12,5 \%$ \\
[b1) darunter: in der Firma] & {$[8]$} & {$[6]$} & {$[0]$} \\
\hline \multirow{2}{*}{ c) Verfahren ohne dokumentierte Kontakte } & {$[13,8 \%]$} & {$[14,0 \%]$} & {$[0,0 \%]$} \\
\hline \hline \multirow{2}{*}{ Verfahren gesamt } & $24,1 \%$ & $14,0 \%$ & $20,8 \%$ \\
\hline
\end{tabular}

* Aus Vergleichsgründen im Süden LRA und zugehöriges GAA.

\subsubsection{Gegenstände der Kommunikation}

Die Phase der Antragsbearbeitung dient der Abklärung rechtlicher und tatsächlicher Grundlagen der anstehenden Genehmigungsentscheidung. Die damit verbundenen wichtigsten Gegenstände und Probleme, die Anlaß für Kontakte zwischen Antragsteller und Behörden waren, sind in Tab. $19 \mathrm{zu}-$ sammengestellt. 


\section{Tab. 19}

Gegenstände der Kommunikation (Auswahl) im Zeitraum der Antragsbearbeitung

\begin{tabular}{|l|c|c|c|}
\cline { 2 - 4 } \multicolumn{1}{c|}{} & $G A A-N$ & $L R A-I$ & $L R A-2$ \\
\hline a) Nachforderungen Antragsunterlagen & 21 & 30 & 12 \\
& $36,2 \%$ & $69,8 \%$ & $50,0 \%$ \\
[a1) darunter: nur Nachforderungen vom & {$[3]$} & {$[12]$} & {$[7]$} \\
GAA als Fachbehörde] & {$[5,2 \%]$} & {$[27,9 \%]$} & {$[29,2 \%]$} \\
[a2) darunter: nur Nachforderungen von & {$[17]$} & {$[12]$} & {$[3]$} \\
sonstigen Fachbehörden] & {$[29,3 \%]$} & {$[27,9 \%]$} & {$[12,5 \%]$} \\
[a3) darunter: Nachforderungen vom & {$[3]$} & {$[6]$} & {$[2]$} \\
GAA und von sonstigen Fachbehörden] & {$[5,2 \%]$} & {$[14,0 \%]$} & {$[8,3 \%]$} \\
[a4) darunter: Einforderung gutachtlicher & {$[4]$} & {$[3]$} & {$[2]$} \\
Prüfbelege/Meßnachweise] & {$[6,9 \%]$} & {$[7,0 \%]$} & {$[8,3 \%]$} \\
\hline b) Befristete Anmahnungen & 6 & 12 & 5 \\
\multicolumn{1}{|c|}{ nachgeforderter Unterlagen } & $10,3 \%$ & $27,9 \%$ & $20,8 \%$ \\
\hline c) Sachliche Kontroversen & 36 & 38 & 19 \\
& $62,1 \%$ & $88,4 \%$ & $79,2 \%$ \\
\hline d) Informeller Genehmigungsbescheid & 29 & 2 & 0 \\
$\quad$ (Entwurf) & $50,0 \%$ & $4,7 \%$ & $0,0 \%$ \\
\hline e) Anmahnung Verfahrensbeschleunigung & 8 & 5 & 2 \\
\hline seitens Antragsteller & $13,8 \%$ & $11,6 \%$ & $8,3 \%$ \\
\hline \hline Verfahren gesamt* & 58 & 43 & 24 \\
\hline
\end{tabular}

* Da die Kategorien eine Auswahl der Beratungsgegenstände darstellen und sich zudem nicht wechselseitig ausschließen (Mehrfachnennungen), übersteigt die Summe der absoluten bzw. relativen Häufigkeiten die jeweilige Grundgesamtheit der Verfahren bzw. 100\%.

Von seiten der Behörden ging es dabei naheliegenderweise vordringlich um die Komplettierung der Antragsunterlagen (Kategorie a), zumal in diese Verfahrensphase auch die förmliche Beteiligung der in ihren Belangen betroffenen Fachbehörden mit der Aufforderung zur Abgabe einer Stellungnahme fällt. Antragsbezogene Nachforderungen ergingen im Süden in deutlich mehr Verfahren als im Norden, wobei der Anteil beim LRA-1 mit $69,8 \%$ nahezu doppelt so hoch ausfällt wie beim GAA-N mit lediglich $36,2 \% .{ }^{160}$ Der Unterschied ist hochsignifikant. ${ }^{161}$ Seine modellbedingten

160 Beim LRA-2 liegt der entsprechende Anteil mit 50,0\% über dem des GAA-N und unter dem des LRA-1. Die jeweiligen Anteilsdifferenzen sind statistisch nicht signifikant $\left(\mathrm{chi}^{2}=2,56\right.$ bzw. 1,29 , jeweils $<2,71$ für $\mathrm{p}=0,1$ und $\left.\mathrm{df}=1\right)$.

$161 \mathrm{chi}^{2}=11,16>10,82, \mathrm{p}=0,001, \mathrm{df}=1$. 
Ursachen erhellt eine Aufschlüsselung nach der fachlichen Zuständigkeit der Behörden, die die Nachforderungen erhoben haben (Kategorien im Kleindruck a1 bis a3): ${ }^{162}$ Das GAA als für den Immissionsschutz zuständige Fachbehörde in Gestalt des GAA-N im Norden bzw. des GAA-1 und GAA-2 im Süden einerseits und die »sonstigen«, nicht-immissionsschutzrechtliche Belange vertretenden Fachbehörden andererseits. Während letztere beim GAA-N und LRA-1 mit ähnlichen Anteilen von 34,5\% bzw. 41,9\% (20 bzw. 18 absolut) der Verfahren Unterlagen nachgefordert haben (Kategorien a2 plus a3), ${ }^{163}$ klaffen die Häufigkeiten bei den Nachforderungen des GAA hochsignifikant auseinander (Kategorien a1 plus a3). Für das GAA-N beläuft sich der entsprechende Anteil auf lediglich 10,3\%, für das GAA-1 dagegen mit 41,9\% auf das Dreifache. ${ }^{164}$ Das auf seine Fachkompetenz beschränkte baden-württembergische GAA-1 schließt mit dieser Quote an Nachforderungen zudem ziemlich genau auf zu derjenigen der sonstigen Fachbehörden. Das verweist auf eine nicht nur formell, sondern auch faktisch ähnliche Position in der Verfahrensbeteiligung. Eine privilegierte, auch informale Einflußnahme auf den Entscheidungsgang wird dem baden-württembergischen GAA von seiten des LRA nicht eingeräumt bzw. ersteres scheint selbst (auch) kein Interesse daran zu haben. ${ }^{165}$

Die Befunde sind vor dem Hintergrund zu sehen, daß beim LRA-1 in weitaus geringerem Maße als beim GAA-N Nachforderungen bereits im Rahmen der Vollständigkeitsprüfung der Unterlagen nach Antragseingang erhoben worden sind (vgl. oben Ziff. 2.1). Entsprechend verlagert sich die Vervollständigung eines nicht unerheblichen Teils speziell umweltschutzrelevanter Antragsunterlagen in den Zeitraum der Antragsbearbeitung. Wenn das LRA-1 allein schon in seiner Entscheidung über den fachlichen Informationsbedarf zur Anlagenausstattung weitgehend auf den Sachverstand der Fachbehörden und unter diesen gerade auch auf den des GAA angewiesen ist, verweist dies auf die engen Grenzen, die der faktischen Handlungsautonomie einer primär durch Verwaltungskompetenz legitimierten Genehmigungsinstanz gezogen sind.

162 Ergänzend sei erwähnt, daß Nachforderungen von Antragsunterlagen überproportional - allerdings nicht in statistisch signifikantem Ausmaß - Änderungsgenehmigungs- gegenüber Neugenehmigungsverfahren betrafen. Das gilt sowohl beim GAA-N als auch beim LRA-1 und zwar mit nahezu gleichen proportionalen Relationen.

163 Beim LRA-2 ergibt sich ein in der Höhe von den anderen beiden Genehmigungsbehörden deutlich (nach unten) abweichender Anteil von nur 20,8\%. Die Differenz zum GAA-N i.S. des Modellvergleichs ist nicht signifikant (chi $\left.{ }^{2}=1,47<2,71\right)$, wohl aber (auf niedrigem Niveau) die zum LRA-1, also innerhalb desselben Modells (chi ${ }^{2}$ $=3,02>2,71$ ), Testwerte jeweils für $\mathrm{p}=0,1$ und $\mathrm{df}=1$.

$164 \mathrm{chi}^{2}=13,61>10,82, \mathrm{p}=0,001, \mathrm{df}=1$. - Beim LRA-2 fällt der Anteil mit 37,5\% ähnlich hoch aus wie beim LRA-1.

$165 \mathrm{Zu}$ weiteren Belegen für diese These vgl. unten Ziff. 3.1.1 u. 3.1.2 sowie Ziff. 3.3. 
In Anbetracht des erheblich höheren »Nachholbedarfs« des baden-württembergischen gegenüber dem niedersächsischen GAA bei der Unterlagenvervollständigung mag es überraschen, daß dabei bei beiden Ämtern das Einfordern gutachtlicher Prüfbelege bzw. Meßnachweise mit Anteilen von jeweils weniger als $10 \%$ der Verfahren eine ähnlich geringe, kaum ins Gewicht fallende Rolle spielt (Kategorie a4 im Kleindruck). Die große Masse der Nachforderungen galt also »Selbstauskünften« des Antragstellers über Anlagenausstattung und Betriebsabläufe. Das deutet darauf hin, daß sich die Entscheidung, ob der Betreiber durch Dritte (Gutachter) »objektivierte« Nachweise beizubringen hat, im wesentlichen nach standardisierten, von der konkreten Ausgestaltung der Anlage und ihres Betriebs weitgehend unabhängigen Kriterien richtet und weniger nach fallspezifischen »Verdachtsmomenten« unzulänglichen Umweltschutzes bzw. unzureichender Umweltschutzprophylaxe. Entsprechend wurde die Mehrzahl derartiger Nachweise im Norden wie im Süden bereits frühzeitig im Rahmen der Vorantragsberatung gefordert (vgl. oben Ziff. 1.3.4), beim GAA-N außerdem in zwei Fällen ohne Vorantragsberatung im Rahmen der Vollständigkeitsprüfung nach Antragseingang (vgl. oben Ziff. 2.1, Anm. 101).

Dem unterschiedlichen Umfang von Antragsergänzungen dürfte der weitere Befund zuzurechnen sein, daß beim LRA-1 im Vergleich zum GAA-N aüch signifikant häufiger befristete Anmahnungen der nachzureichenden Unterlagen mit einem Anteil von 27,9\% gegenüber einem Anteil von 10,3\% der Verfahren (Kategorie b) ergingen. ${ }^{166}$ Aus der insoweit »stimmigen « Relation folgt aber auch, daß das Genehmigungsmodell keinen belangvollen Einfluß auf die Säumigkeit der Antragsteller hat.

Mehr noch als die (informationelle) Komplettierung der Antragsunterlagen beschäftigten sachliche Kontroversen um die tatsächlichen und rechtlichen Anforderungen an die Genehmigungserteilung die Verfahrensbeteiligten (Kategorie c). Der Anteil solcher (streckenweise) kontrovers geführten Verfahren liegt beim LRA-1 mit 88,4\% erheblich über dem von $62,1 \%$ beim GAA-N. ${ }^{167}$ Ging insoweit dort nur etwa jedes achte bis neunte (genau: $8,6$.$) Verfahren »glatt« über die Bühne, verlief hier jedes zweite bis dritte$ (genau: 2,6.) Verfahren für die Behörden insoweit »problemlos«. Die Differenz ist hochsignifikant. Auch in dieser Hinsicht weist eine nähere Aufschlüsselung der kontroversen Verfahren nach ihren Streitgegenständen und den daran Beteiligten auf die modellbedingte institutionelle Trennung von Genehmigungs- und zentraler Fachkompetenz als Hauptursache hin (s. dazu näher unten Ziff. 3.1). ${ }^{168}$

$166 \mathrm{chi}^{2}=5,28>5,02, \mathrm{p}=0,025, \mathrm{df}=1$.

$167 \mathrm{chi}^{2}=8,74>7,88, \mathrm{p}=0,005, \mathrm{df}=1$.

168 Das gilt auch für das LRA-2. Sein Anteil an kontroversen Verfahren mit 79,2\% liegt zwar um 9,2 Prozentpunkte unter dem entsprechenden Wert des LRA-1, deutlich 
Ein weiterer Gegenstand der Kommunikation in der Phase der Antragsbearbeitung war die Unterrichtung des Antragstellers durch die Genehmigungsbehörde über die (unter Berücksichtigung der fachbehördlichen Stellungnahmen) vorgesehene Genehmigungsentscheidung in Form eines schriftlichen Entwurfs (Kategorie d). Wie ein Blick auf diese Kategorie zeigt, handelt es sich dabei um eine weitgehend auf das niedersächsische GAA-N beschränkte, hier in der Hälfte der Verfahren zum Zuge gekommene Praxis. Im Süden bildet ein solcher »Service« mit nur zwei Fällen beim LRA-1 eine seltene Ausnahme (und dort auch erst - im Unterschied zum GAA-N - auf ausdrückliches Ersuchen des Antragstellers). Mit der informellen Bekanntgabe des geplanten Genehmigungsinhalts gibt das GAA$\mathrm{N}$ dem Antragsteller Gelegenheit (oder auch Ermutigung), noch vor offiziellem Abschluß des Verfahrens Einwendungen gegen einzelne, ihn beschwerende Bestimmungen und Auflagen anzumelden und für Abänderungen zu seinen Gunsten einzutreten. Wieweit es sich dabei um einen modellbedingten Unterschied handelt und was daraus für das Verfahrensergebnis am Ende folgt, wird in einem späteren Abschnitt (Ziff. 3.2) zu erörtern sein. Im vorliegenden Zusammenhang sei lediglich vorweggenommen, daß bei kontroversem Verfahrensverlauf häufiger als in unstreitigen Fällen dem Antragsteller vorab eine Entwurfsfassung zuging. ${ }^{169}$

Angesichts der in der einschlägigen Literatur nahezu durchgängig geführten Klage über zu lange Genehmigungsdauern, die den Antragsteller vielfach davon abhielten, genehmigungspflichtige Investitionen zu tätigen, sind in den hier untersuchten Verfahren (aktenkundige) Beschwerden über behördliche Verzögerungen bzw. Anmahnungen einer Verfahrensbeschleunigung ${ }^{170}$ bei allen drei Genehmigungsbehörden nur in vergleichsweise geringem Umfang von rd. 10\% der Verfahren aufgetreten (Kategorie e). Daraus läßt sich zum einen schließen, daß in der großen Mehrheit der vereinfachten Verfahren in beiden Genehmigungsmodellen zumindest aus Sicht des Antragstellers die Bearbeitungsdauer seines Antrags seinen eigenen

größer fällt aber der Abstand zum GAA-N mit einer Differenz von 17,1 Prozentpunkten aus. Vgl. dazu im einzelnen unten Ziff. 3.1.1.

169 Die Differenz verfehlt allerdings, wenn auch knapp, die statistische Signifikanzgrenze $\left(\mathrm{chi}^{2}=2,64<2,71\right.$ für $\mathrm{p}=0,1$ und $\left.\mathrm{df}=1\right)$.

170 Dabei wurde die Kategorie der »Anmahnung « sehr weit gefaßt. Sie schließt auch bloße Anfragen des Antragstellers nach dem Stand des Verfahrens mit ein. - Für die Möglichkeit, daß es sich bei der geringen Zahl der registrierten Beschwerden und Anmahnungen um ein der Erhebungsgrundlage (Akten der Genehmigungsbehörde) zuzuschreibendes Forschungsartefakt handelt, spricht wenig. Wie auch die Befunde zur Verfahrensdauer zeigen werden, hat die verfahrensleitende Genehmigungsbehörde kaum Anlaß, solche Vorhaltungen nicht aktenkundig zu machen. Eher dürfte das Gegenteil in ihrem Interesse liegen. Schließlich waren es neben dem Antragsteller selbst hauptsächlich die beteiligten Fachbehörden, die durch zögerliche Abgaben ihrer Stellungnahmen Verfahrensverlängerungen verursachten (s. unten Ziff. 4.3.3.2 und Ziff. 4.4). 
terminlichen Interessen an der Genehmigungserteilung nicht zuwiderläuft. Vielmehr vermittelt eine Reihe der hier dargestellten Untersuchungsbefunde im Gegenteil den Eindruck, ${ }^{171}$ daß einer nicht unerheblichen Anzahl der Antragsteller an einem zügigen Verfahrensgang offenbar wenig gelegen war, wovon nicht zuletzt die häufig nur schleppend und erst nach mehrmaligen Anmahnungen beigebrachten Antragsunterlagen zeugen. Auch im übrigen herrscht der Eindruck einer eher reaktiven als aktiven Kooperationsbereitschaft vor. Schließlich zeigten gerade diejenigen Betreiber, die die genehmigungspflichtigen Änderungen bereits durchgeführt hatten oder auf Druck der Behörden (unfreiwillig) aus Sanierungserfordernissen durchführen mußten, kaum ein Eigeninteresse an einer raschen Genehmigungserteilung. Sie richteten ihr Augenmerk vielmehr vorrangig auf Zeitgewinn und möglichst günstige Genehmigungsbedingungen. Die Behörden machten es ihnen dabei insofern leicht, als sie es - von wenigen Ausnahmen abgesehen (vgl. oben Ziff. 1.3.4) - vorzogen, den Weiterbetrieb der geänderten oder zu ändernden Anlage trotz fehlender gültiger Genehmigung zu dulden, als »harte« Zwangsmaßnahmen wie Betriebsuntersagung oder Stillegung glaubhaft anzudrohen oder gar zu ergreifen, wenn nur der Betreiber sich bereit erklärte, den erforderlichen Änderungsgenehmigungsantrag zu stellen.

Eine genauere Betrachtung des Verlaufs der Fälle, in denen der Antragsteller gegenüber der Genehmigungsbehörde auf eine Beschleunigung des Verfahrens dringt, führt zu der - auf Grund der geringen Fallzahl allerdings hier nicht weiter abzusichernden - Hypothese, daß das Argument einer primär der Verwaltung anzulastenden zu langen Verfahrensdauer über den so erzeugten Zeitdruck im Kern auf eine Absenkung der inhaltlichen Standards für die Genehmigungserteilung zugunsten der Betreiber zielt. Von einer einzigen Ausnahme (beim GAA-N) abgesehen, hatte der anmahnende Antragsteller außerdem auch Einwände gegen behördliche Anforderungen erhoben, deren »Verhandlung « zwangsläufig zusätzliche Zeit in Anspruch nahm. ${ }^{172}$

\subsubsection{Behördliche Betriebsbegehungen bzw. -kontrollen}

Geht man davon aus, daß persönliche Inaugenscheinnahmen der zur Genehmigung anstehenden Anlage (bei Änderungsgenehmigungen) und ihrer be-

171 Vgl. oben Ziff. 1.2, 1.3.4 u. 2.1.

172 Wenn auch im Unterschied zum GAA-N beim LRA-1 fast $90 \%$ der Verfahren kontrovers geführt wurden und von daher allein die statistische Wahrscheinlichkeit dafür spricht, daß auch die Beschwerdefälle in diese Gruppe fallen, so steht dies nicht der hier vertretenen generellen These entgegen, daß es dem Antragsteller in erster Linie um eine für ihn günstige und nicht unbedingt um eine schnelle Genehmigung geht. 
trieblichen und außerbetrieblichen Umgebung (bei Änderungs- und Neugenehmigungen) die behördlichen Entscheidungsgrundlagen wenn nicht verbessern, so doch zumindest absichern und damit auch der materiellen Qualität der Genehmigungsentscheidung zugute kommen dürften, ist die Frage von Interesse, ob bzw. inwieweit die Häufigkeit von Betriebsbegehungen bzw. -kontrollen in den beiden Genehmigungsmodellen differiert.

Tab. 20 gibt die entsprechenden Befunde für den Zeitraum der Antragsbearbeitung wieder. Danach wurden beim LRA-1 mit einem Anteil von einem guten Drittel $(34,9 \%)$ der Verfahren die Betriebe häufiger in Augenschein genommen als beim GAA-N mit einem Anteil von einem guten Fünftel $(22,4 \%)$. Die Differenz von 12,5 Prozentpunkten bleibt allerdings unterhalb der statistischen Signifikanzgrenze. ${ }^{173}$ Im übrigen waren es bei beiden Behörden jeweils nur einige wenige Betriebe, die im Zuge der Antragsbearbeitung nochmals aufgesucht wurden (Zeile im Kleindruck). ${ }^{174}$

Tab. 20

Verfahren mit Betriebsbegehungen bzw. -kontrollen (Inspektionen) durch die Genehmigungsbehörde* im Zeitraum der Antragsbearbeitung

\begin{tabular}{|l|c|c|c|}
\cline { 2 - 4 } \multicolumn{1}{c|}{} & $G A A-N$ & LRA-I & LRA-2 \\
\hline Verfahren mit Inspektionen & 13 & 15 & 4 \\
[darunter: Fälle mit mehr als einer Inspektion] & $22,4 \%$ & $34,9 \%$ & $16,7 \%$ \\
& {$[4]$} & {$[4]$} & {$[1]$} \\
& {$[6,9 \%]$} & {$[9,3 \%]$} & {$[4,2 \%]$} \\
\hline \multirow{2}{*}{ Verfahren ohne Inspektionen } & 45 & 28 & 20 \\
\hline \hline \multirow{2}{*}{ Verfahren gesamt } & $77,6 \%$ & $65,1 \%$ & $83,3 \%$ \\
\hline
\end{tabular}

* Aus Vergleichsgründen im Süden LRA und zugehöriges GAA.

Veranlassung und Durchführung der Inspektionen lagen im Süden, wie auch schon in den analogen Fällen im Vorantragszeitraum, überwiegend in der Hand des zuständigen GAA, wobei in gut der Hälfte der Fälle auch das LRA-1 zugegen war. ${ }^{175}$ Lediglich in vier Fällen nahm das LRA-1 allein (1) bzw. unter Beteiligung sonstiger Fachbehörden (3) den Betrieb in Augen-

$173 \mathrm{chi}^{2}=1,94<2,71$ für $\mathrm{p}=0,1$ und $\mathrm{df}=1$.

174 Beim GAA-N in je zwei Fällen zwei- bzw. dreimal, beim LRA-1 in drei Fällen zweimal, in einem Fall viermal, beim LRA-2 in einem Fall zweimal.

175 Beim LRA-2 trifft das nur in einem der vier einschlägigen Fälle zu. 
schein. Im Norden war die Anwesenheit des GAA-N auf Grund seiner Doppelfunktion als Fach- und Genehmigungsbehörde zwangsläufig stets gegeben, in zwei Fällen mit zusätzlicher Teilnahme sonstiger Fachbehörden. ${ }^{176}$ Die Beiziehung von Sachverständigen anläßlich einer vom Antragsteller vorzulegenden umweltschutzbezogenen Begutachtung bildete - ähnlich wie die Beteiligung sonstiger Fachbehörden - eher die Ausnahme. Beim LRA-1 waren es drei, beim GAA-N zwei Fälle.

Betrachtet man den gesamten, sich über beide hier unterschiedene Phasen vor bzw. nach formeller Antragstellung erstreckenden Zeitraum des Verfahrensverlaufs (Tab. 21), so waren beim LRA-1 in mehr als der Hälfte der Verfahren $(53,5 \%)$ die betrieblichen Verhältnisse durch unmittelbare Präsenz der Genehmigungsbehörde, teils »vertreten « durch das zugehörige GAA, bei Genehmigungserteilung bekannt. Beim GAA-N fällt der entsprechende Anteil mit 46,6\% der Verfahren auch hier kleiner aus, der Unterschied ist aber nicht signifikant. ${ }^{177}$ Bei diesem Vergleich ist allerdings zu berücksichtigen, daß der Anteil beim GAA-N - im Unterschied zum LRA1 - fünf Fälle enthält, die sich einer verfahrensunabhängigen, generellen Betriebsrevision im Rahmen eines amtsinternen Arbeitsschwerpunkts verdanken (vgl. oben Ziff. 1.3.3). Keiner dieser Betriebe wurde nach der Revision, deren Ergebnis das Verfahren dann auch erst auslöste, im weiteren Verlauf einer nochmaligen Prüfung unterworfen.

Die in den Tab. 20 und 21 dargestellten Häufigkeiten von Betriebskontrollen sind auf die davon betroffenen Verfahren bezogen. Mehrfachkontrollen ein und desselben Betriebs sind darin zwangsläufig nicht berücksichtigt. Sie lassen sich erst mit einer von der Verfahrenseinheit unabhängigen Zählung der in beiden Verfahrensphasen durchgeführten Betriebsüberprüfungen erfassen.

176 Im Norden wie im Süden haben außerdem auch Ortstermine der Fachbehörden ohne Anwesenheit des GAA-N bzw. LRA-1 oder GAA-1 stattgefunden. Es handelt sich dabei jedoch jeweils nur um wenige Fälle, die durch vorherige oder nachträgliche Unterrichtung der Genehmigungsbehörde dort aktenkundig geworden sind. Im übrigen liefern die dokumentierten Verfahrensverläufe keine Anhaltspunkte dafür, daß darüber hinaus - d.h. an der Genehmigungsbehörde vorbei und insofern für die vorliegende Untersuchung nicht erkennbar - die Fachbehörden eigenmächtig solche Termine abgehalten haben.

177 chi $^{2}=0,46<2,71$ für $\mathrm{p}=0,1$ und $\mathrm{df}=1$. - Das LRA-2 hebt sich mit einem Gesamtanteil an Betriebskontrollen von lediglich einem Fünftel der Verfahren (20,8\%) von den beiden anderen Genehmigungsbehörden statistisch signifikant ab (in bezug auf GAA-N: chi $^{2}=4,73>3,84, p=0,05$, in bezug auf LRA-1: chi $^{2}=6,75>6,64, p=$ 0,01 , jeweils $\mathrm{df}=1$ ). 
Tab. 21

Verfahren mit Betriebsbegehungen bzw. -kontrollen (Inspektionen) durch die Genehmigungsbehörde* ab Vorantragszeitraum bis Genehmigungsbescheid

\begin{tabular}{|l|c|c|c|}
\cline { 2 - 4 } \multicolumn{1}{c|}{} & GAA-N & LRA-1 & LRA-2 \\
\hline Verfahren mit Inspektionen & 27 & 23 & 5 \\
& $46,6 \%$ & $53,5 \%$ & $20,8 \%$ \\
\hline \multirow{2}{*}{ Verfahren ohne Inspektionen } & 31 & 20 & 19 \\
\hline \hline \multirow{2}{*}{ Verfahren gesamt } & $53,4 \%$ & $46,5 \%$ & $79,2 \%$ \\
\hline
\end{tabular}

* Aus Vergleichsgründen im Süden LRA und zugehöriges GAA.

Wie aus der zugehörigen Tab. 21.1 hervorgeht, erfolgten beim GAA-N und LRA-1 in nahezu gleicher (absoluter) Anzahl (38- bzw. 36mal) betriebliche Kontrollen (Zeile 2). Bezieht man diese Werte auf die jeweils zugehörige Grundgesamtheit der Genehmigungsverfahren (erste Zeile), ergeben sich die im Durchschnitt pro Verfahren vorgenommenen Inspektionen (letzte Zeile). Danach liegt der Durchschnittswert beim LRA-1 mit 0,84 Kontrollen pro Verfahren deutlich über dem beim GAA-N mit 0,66 Kontrollen pro Verfahren.

Tab. 21.1

Anzahl der Betriebsbegehungen bzw. -kontrollen (Inspektionen) durch die Genehmigungsbehörde* ab Vorantragszeitraum bis Genehmigungsbescheid

\begin{tabular}{|l|c|c|c|}
\cline { 2 - 4 } \multicolumn{1}{c|}{} & GAA-N & LRA-1 & LRA-2 \\
\hline Zahl der Genehmigungsverfahren & 58 & 43 & 24 \\
\hline Zahl der Inspektionen & 38 & 36 & 9 \\
\hline Inspektionen pro Verfahren & 0,66 & 0,84 & 0,38 \\
\hline
\end{tabular}

* Aus Vergleichsgründen im Süden LRA und zugehöriges GAA. 
Ein weiterer Unterschied zwischen GAA-N und LRA-1 tritt zutage, wenn man die Unternehmensgröße der kontrollierten Betriebe einbezieht. Während beim GAA-N alle drei Größenklassen der verfahrensführenden Unternehmen in etwa entsprechend ihrem jeweiligen Anteil in der Grundgesamtheit ${ }^{178}$ betrieblichen Überprüfungen unterzogen wurden, ${ }^{179}$ sind beim LRA-1 in dieser Hinsicht die größeren Firmen unterrepräsentiert ${ }^{180}$ und die kleineren Firmen (noch ausgeprägter) überrepräsentiert. ${ }^{181}$ An diesen Befund läßt sich die These anschließen, daß das baden-württembergische Genehmigungsmodell sich eher als das niedersächsische $»$ Rücksichtnahmen « auf regionale/lokale Wirtschaftsinteressen öffnet, denen sich auch das dortige GAA trotz seiner insoweit gegebenen größeren (»kommunalpolitischen«) Unabhängigkeit nur begrenzt widersetzt.

Den modellbedingt unterschiedlichen formellen Entscheidungskompetenzen des GAA dürfte ein weiterer Befund zuzurechnen sein. Er betrifft den Zusammenhang zwischen Betriebskontrollen und streitigem Verfahrenshergang. ${ }^{182}$ Zwar wurden bei beiden Genehmigungsmodellen in (sachlich) kontrovers verlaufenen Fällen die Betriebe insgesamt nicht häufiger geprüft als in kontroversfreien. ${ }^{183}$ Schlüsselt man aber die kontroversen Fälle nach ihrem Bezug zu immissionsschutzrechtlichen Problemen i.e.S. auf, stellt sich folgendes heraus. ${ }^{184}$ Beim GAA-N sind Betriebskontrollen überproportional häufig in den Verfahren zü finden, iñ deneñ die Streitigkeiten ausschließlich oder zumindest auch sonstige, nicht-immissionsschutzrechtliche fachliche Belange betrafen. ${ }^{185}$ Demgegenüber sind Ver-

178 Zur Verteilung der Unternehmensgröße in den Grundgesamtheiten der Genehmigungsverfahren aller drei Genehmigungsbehörden vgl. oben Kap. II, Ziff. 4.2.2, Tab. 4.

179 Vgl. im einzelnen die entsprechenden Kreuztabellierungen für alle drei Genehmigungsbehörden in Tab. 21a-A im Anhang. Der Testwert beispielsweise für die hier besonders interessierenden größeren Unternehmen liegt weit unter einer möglichen Signifikanz mit chi ${ }^{2}=1,73<2,71$ für $\mathrm{p}=0,1$ und $\mathrm{df}=1$. Er bleibt auch dann noch klar unterhalb der Signifikanzgrenze $\left(\mathrm{chi}^{2}=2,32<2,71\right)$, wenn man die (nur beim GAA-N vorkommenden) fünf Revisionsfälle, die drei größere und zwei mittlere Firmen betreffen, außer Betracht läßt.

$180 \mathrm{chi}^{2}=2,72>2,71, \mathrm{p}=0,1, \mathrm{df}=1$.

$181 \mathrm{chi}^{2}=8,66>7,88, \mathrm{p}=0,005, \mathrm{df}=1$. - Beim LRA-2 unterbleibt wegen der (absoluten) Fallzahl von nur 5 Betriebskontrollen die Prüfung eines möglichen Zusammenhangs mit der Unternehmensgröße.

182 Zum Umfang streitiger Verfahrensverläufe vgl. oben Ziff. 2.4.4 mit Tab. 19, Kategorie c sowie ausführlicher unten Ziff. 3.1.1.

$183 \mathrm{Zu}$ den Werten im einzelnen vgl. die zugehörigen Kreuztabellierungen in Tab. 21bA im Anhang.

184 Aus dem oben in Anm. 181 genannten Grund bleibt dabei LRA-2 außer Betracht.

185 Der Zusammenhang ist statistisch signifikant: chi $^{2}=4,26>3,84, p=0,05, \mathrm{df}=1$. Die Zahl der dieser Kategorie zugehörigen Fälle ergibt sich aus der Addition der Spalten 1 u. 3 von Tab. 21b-A. 
fahren, die entweder ausschließlich oder zumindest auch mit immissionsschutzrechtlichen Kontroversen einhergingen, eher (wenn auch nur leicht, also statistisch unerheblich) bei den Kontrollen unterrepräsentiert. ${ }^{186}$ Beim LRA-1 zeigt sich für die beiden nach ihrem Konfliktbezug unterschiedenen Kategorien von Verfahren ein gegenläufiger Zusammenhang. Die Fälle mit Konflikten (auch) um nicht-immissionsschutzrechtliche Belange sind hier zwar bei den Betriebskontrollen ebenfalls überrepräsentiert, die Relation ist aber nicht statistisch signifikant. ${ }^{187}$ In größerem und statistisch signifikantem Maße (wenn auch knapp und auf niedrigem Niveau) trifft das jedoch auf die Verfahren mit immissionsschutzrechtlichen Streitigkeiten zu. ${ }^{188}$ Dieser Zusammenhang ${ }^{189}$ stützt die These weiter ab, daß ein GAA, das sich mangels Genehmigungskompetenz allein über seine spezifischen fachtechnischen Mittel im Verfahren zur Geltung bringen kann, auf diese in stärkerem Maße zurückgreift als sein mit beiden zentralen Kompetenzen ausgestattetes Pendant.

Ergänzend sei noch erwähnt, daß ein Zusammenhang zwischen Betriebskontrollen und Genehmigungsart, den zu vermuten plausible Gründe durchaus nahelegten, nicht festzustellen ist. Die betrieblichen Überprüfungen betrafen beim GAA-N wie beim LRA-1 nur unwesentlich häufiger Neu- als Änderungsgenehmigungen. ${ }^{190}$

186 Die Zahl der dieser Kategorie zugehörigen Fälle ergibt sich aus der Addition der Spalten 2 u. 3 von Tab. 21b-A. Fälle, in denen der Konfliktstoff ausschließlich immissionsschutzrechtlicher Natur war (Spalte 2), sind sogar deutlich unterrepräsentiert.

$187 \mathrm{chi}^{2}=1,77<2,71$ für $\mathrm{p}=0,1$ und $\mathrm{df}=1$.

$188 \mathrm{chi}^{2}=2,72>2,71, \mathrm{p}=0,1, \mathrm{~d} \mathbf{f}=1$. - Der überproportionale Anteil der Betriebskontrollen in beiden Verfahrenskategorien ist vor dem Hintergrund zu sehen, daß sich die Kategorien nicht wechselseitig ausschließen wie eben auch das Aufkommen eines immissionsschutzrechtlichen Konflikts nicht das (zusätzliche) Auftreten eines nicht-immissionsschutzrechtlichen Belangen geltenden Konflikts - und umgekehrt - ausschließt. Hinzu kommt der hier insgesamt hohe Anteil an Konfliktfällen von fast $90 \%$ der Verfahren. Von daher kommt nicht der Überproportionalität als solcher, sondern ihrem Ausmaß die wesentliche Aussagekraft in der vorliegenden Zusammenhangsanalyse zu.

189 Eine Verzerrung dieses Ergebnisses durch die Unternehmensgröße, für die sich, wie gezeigt, beim LRA-1, nicht aber beim GAA-N ein systematischer Zusammenhang mit Betriebskontrollen herausgestellt hat und die von daher als mögliche intervenierende Variable in Frage kam, ist nicht festzustellen. Wie aus Tab. 23b-A im Anhang hervorgeht, verteilen sich die Streitfälle bzw. ihre hier unterschiedenen Unterkategorien auf die drei Unternehmensgrößenklassen beim GAA-N wie beim LRA1 mit prozentual nur geringfügigen, weit unter jeglicher statistischer Signifikanz liegenden Abweichungen von den jeweiligen Anteilen in der Grundgesamtheit.

190 Beim LRA-2 betrafen die vergleichsweise und auch absolut wenigen (vgl. oben Anm. 181) Betriebskontrollen allerdings ausschließlich Neugenehmigungen. 


\section{Entscheidungsverlauf}

Galt die bisherige Darstellung der Analyse des äußeren Verfahrensverlaufs nach Maßgabe der Aktivitäten der Hauptbeteiligten, behandelt das vorliegende Kapitel die inhaltlichen Dimensionen des Entscheidungsprozesses sowie die im Genehmigungsbescheid niedergelegten materiellen Ergebnisse. Da von der Anlage der Untersuchung her in allen Verfahren die Genehmigung erteilt wurde, ist der Verfahrensausgang einer (förmlichen) Ablehnung der Genehmigungserteilung ausgeschlossen.

Eine solche förmliche Ablehnung hat die Genehmigungsbehörde nach den gesetzlichen Bestimmungen auszusprechen, »sobald die Prüfung ergibt, daß die Genehmigungsvoraussetzungen nicht vorliegen und ihre Erfüllung nicht durch Nebenbestimmungen sichergestellt werden kann.« Sie »soll« dies tun, »wenn der Antragsteller einer Aufforderung zur Ergänzung der Unterlagen innerhalb einer ihm gesetzten Frist, die auch im Falle ihrer Verlängerung drei Monate nicht überschreiten soll, nicht nachgekommen ist. « ${ }^{191}$ Die hier untersuchten Verfahrensverläufe lassen es allerdings als wenig wahrscheinlich erscheinen, daß die gesetzlich vorgesehenen Ablehnungsmöglichkeiten in der Genehmigungspraxis, von Extremfällen abgesehen, auch tatsächlich zum Zuge kommen. Die Behörden sehen darin eher ein Drohpotential. Doch selbst in dieser Funktion kam es nür selten zur Añwendung. Statt dessen griff man eher auf Androhung und ggf. auch Vollzug von Verwaltungszwangsmaßnahmen als funktionale Äquivalente zurück, wobei auch diese Mittel eher sparsam verwandt wurden (vgl. oben Ziff. 1.3.4). In aller Regel war den Genehmigungsbehörden im Norden wie im Süden vorrangig daran gelegen, bei Vorhaben, die in der beantragten Konzeption als nicht genehmigungsfähig betrachtet wurden, den Antragsteller von der Notwendigkeit entsprechender (zusätzliche Kosten verursachenden) Nachbesserungen der Anlagenausstattung zu überzeugen bzw. bei umfassendem Änderungsbedarf zu empfehlen, den Antrag zurückzuziehen und in überarbeiteter Form neu einzureichen. ${ }^{192}$ Bei säumiger Unterlagenbeibringung schließlich setzten die Behörden mehr auf den guten Willen als auf strikte Fristvorgaben, zumindest eine gute Weile lang.

Nicht nur das Interesse an rechtskonformen Anlagenzuständen dürfte die Genehmigungsbehörde förmliche Ablehnungen vermeiden lassen. Die Anwendung dieses Instruments bei unzureichender Genehmigungsfähigkeit

$191 \S 20$ Abs. 2 der 9. BImSchV. Die Ablehnung ist nach $\S 20$ Abs. 3 Satz 1 der Verordnung i.V.m. § 10 Abs. 7 BImSchG in Form eines (schriftlichen) Genehmigungsbescheids zu erlassen und schriftlich zu begründen.

192 Da es sich bei der Genehmigung um einen begünstigenden Verwaltungsakt handelt, kann sie sich (aus rechtlicher Sicht) nur auf das erstrecken, was auch beantragt wurde. Die Genehmigungsbehörde kann nicht von sich aus den Antragsgegenstand ändern, im Zweifel müßte sie dann eben den Antrag ablehnen. 
ist für sie auch insofern riskant, als es ihr eine prospektive Begründungslast eines noch in Planung stehenden Vorhabens auferlegt. Demgegenüber kann sie sich bei Stillegungs-, Untersagungs- oder Beseitigungsverfügungen nach $\S 20 \mathrm{BImSchG}$ auf belegbare, tatsächlich gegebene Mißstände beziehen. Vergleichbares gilt für die Ablehnungsvariante bei säumiger Unterlagenbeibringung, wo die Behörde im Zweifel der Nachweispflicht unterliegt, nicht durch eigene (Verfahrens-) Fehler Verzögerungen verursacht oder mit verursacht zu haben.

Kurz gefaßt: Im Genehmigungsverfahren steht nicht die Entscheidung über die Alternative der Erteilung oder Nichterteilung einer Genehmigung an, sondern die Entscheidung über deren inhaltliche Ausgestaltung im Hinblick auf die nach den immissionsschutzrechtlichen Vorschriften erforderliche, spezifische Anlagenausstattung und die vom Betreiber zu erfüllenden anlagenspezifischen Pflichten nach Inbetriebnahme. Hinzu kommen die Entscheidungen über die von den in ihren Belangen betroffenen Fachbehörden erhobenen anlagenspezifischen Forderungen und Betriebsbedingungen, zumal die immissionsschutzrechtliche Genehmigung auf Grund des Konzentrationsprinzips gem. $\S 13 \mathrm{BImSchG}$ andere behördliche Erlaubnisse und Genehmigungen einschließt. In welchem Maße es in den beiden Gruppen entscheidungsbedürftiger Belange, den immissionsschutzrechtlichen i.e.S. und den sonstigen, nicht-immissionsschutzspezifischen, zu Kontroversen zwischen den Verfahrensbeteiligten kam, wie sich »Gewinne « und »Verluste« dabei verteilen, welche Rolle der beim GAA-N geübten Praxis des »Austestens « der Abnahmebereitschaft der projektierten Genehmigungsentscheidung in Form eines Bescheidsentwurfs zukommt, welche Anforderungen am Ende im Genehmigungsbescheid verbindlich fixiert wurden und schließlich wieweit und mit welchem Ausgang das Instrument des Widerspruchs in Anspruch genommen wurde, sind Gegenstand der folgenden Abschnitte.

\subsection{Sachliche Kontroversen zwischen den Hauptbeteiligten}

\subsubsection{Häufigkeit und Art der Kontroversen}

Tab. 22 gibt einen Überblick über die Anzahl an Verfahren, in deren Verlauf es zwischen den Beteiligten zu sachlichen Kontroversen gekommen ist. Dabei werden drei Gruppen von Konfliktkonstellationen unterschieden: Solche, die ausschließlich immissionsschutzrechtliche (Kürzel: I-Streit), solche, die ausschließlich nicht-immissionsschutzrechtliche (Kürzel: NIStreit) sowie solche, die beide Arten von Streitigkeiten (Kürzel: I/NI-Streit) enthalten. 
Tab. 22

Häufigkeiten der Verfahren mit sachlichen Kontroversen zwischen den Hauptbeteiligten

\begin{tabular}{|l|c|c|c|}
\cline { 2 - 4 } \multicolumn{1}{c|}{} & $G A A-N$ & $L R A-1$ & $L R A-2$ \\
\hline a) Verfahren ohne Kontroversen & 22 & 5 & 5 \\
& $37,9 \%$ & $11,6 \%$ & $20,8 \%$ \\
\hline \multirow{2}{*}{ b) Verfahren nur mit NI-Streit } & 14 & 7 & 1 \\
& $24,1 \%$ & $16,3 \%$ & $4,2 \%$ \\
\hline \multirow{2}{*}{ c) Verfahren nur mit I-Streit } & 10 & 14 & 10 \\
\hline \multirow{2}{*}{ d) Verfahren mit I-/NI-Streit } & $17,2 \%$ & $32,6 \%$ & $41,7 \%$ \\
\hline \hline \multirow{2}{*}{ Verfahren gesamt } & 12 & 17 & 8 \\
\hline \hline
\end{tabular}

Wie die Tabelle zeigt, weist das GAA-N mit einem Anteil von 37,9\%, also fast zwei Fünfteln der Verfahren, die höchste Rate an konfliktfreien Verfahren auf, das LRA-1 mit einem Anteil von 11,6\%, also nur einem Zehntel der Verfahren, die niedrigste (Kategorie a). Nicht wesentlich höher fällt die entsprechende Quote mit 20,8\% beim LRA-2 aus. Anders gesagt: In beiden Modellen kam es zwar in mehr als der Hälfte aller Verfahren zu sachlichen Kontroversen, im Süden jedoch mit einem Anteil von 88,4\% (bzw. 79,2\%) weitaus häufiger als im Norden mit einem Anteil von 62,1\% (Kategorien b plus c plus d). ${ }^{193}$ Die Differenz zwischen LRA-1 und GAA-N ist, wie in anderem Zusammenhang bereits erwähnt, hochsignifikant. ${ }^{194}$ Während sich die streitigen Verfahren beim GAA-N zu etwa gleichen Anteilen von jeweils rd. $20 \%$ auf die drei hier unterschiedenen Untergruppen von Kontroversen verteilen, entfällt beim LRA-1 auf diejenige Gruppe, die sowohl mit I- als auch mit NI-Streitigkeiten einherging, mit 39,5\% ein erheblich höheres Kontingent (Kategorie d). ${ }^{195}$

Aus Tab. 22.l ist zu ersehen, daß sich dieser Unterschied in erster Linie dem differierenden Vorkommen von I-Kontroversen verdankt, mit denen (nur oder auch) beim LRA-1 insgesamt $72,1 \%$, beim GAA-N jedoch ledig-

193 Vgl. auch oben Ziff. 2.4.4, Tab. 19, Kategorie c.

194 Vgl. oben Anm. 168. - Die Differenz zwischen LRA-2 und GAA-N in Höhe von 17,1 Prozentpunkten bleibt unterhalb statistischer Signifikanz $\left(\right.$ chi $^{2}=2,04<2,71$ für $\mathrm{p}=0,1$ und $\mathrm{df}=1$ ), was durch die für statistische Prüfzwecke kritische Größe der Grundgesamtheit beim LRA-2 bedingt ist.

195 Auch diese Differenz ist statistisch signifikant: chi $^{2}=4,29>3,84, p=0,05, \mathrm{df}=1$. 
lich $37,9 \%$ der Verfahren einhergingen. Zwar traten auch NI-Konflikte beim LRA-1 mit einer Quote von 55,8\% häufiger auf als beim GAA-N mit einer Quote von 44,8\%, statistisch signifikant (und zwar hochsignifikant) ist aber nur die Differenz bei den I-Kontroversen, nicht aber die bei den NIKontroversen. ${ }^{196}$

\section{Tab. 22.1}

Häufigkeiten der Verfahren mit sachlichen Kontroversen (gruppiert) zwischen den Hauptbeteiligten

\begin{tabular}{|l|c|c|c|}
\cline { 2 - 4 } \multicolumn{1}{c|}{} & GAA-N & LRA-1 & LRA-2 \\
\hline a) Verfahren ohne Kontroversen & 22 & 5 & 5 \\
& $37,9 \%$ & $11,6 \%$ & $20,8 \%$ \\
\hline \multirow{2}{*}{ b) Verfahren (nur bzw. auch) mit NI-Streit } & 26 & 24 & 9 \\
& $44,8 \%$ & $55,8 \%$ & $37,5 \%$ \\
\hline \multirow{2}{*}{ c) Verfahren (nur bzw. auch) mit I-Streit } & 22 & 31 & 18 \\
\hline \hline \multirow{2}{*}{ Verfahren gesamt* } & $37,9 \%$ & $72,1 \%$ & $75,0 \%$ \\
\hline \hline
\end{tabular}

* Da sich die Kategorien (b und c) teilweise überschneiden, liegt die Summe der absoluten und relativen Häufigkeiten über der Gesamtzahl der Verfahren bzw. über 100\%.

Bezogen auf die alternativen Genehmigungsmodelle bedeuten diese Befunde zweierlei. Die baden-württembergische Aufteilung der Genehmigungs- und zentralen immissionsschutzrechtlichen Fachkompetenz auf zwei Instanzen erzeugt nicht allein zusätzlichen Konfliktstoff, was als solches angesichts des insofern erweiterten Kreises der Verfahrensbeteiligten eine voraussehbare Konsequenz ist. Darüber hinaus mündet das zwischen Genehmigungsbehörde und GAA strukturell angelegte Konfliktpotential sehr viel häufiger auch in aktuelle Auseinandersetzungen, als das bei den sonstigen Fachbehörden, bei denen insoweit ein vergleichbares strukturelles Konfliktpotential mit der Genehmigungsbehörde gegeben ist, der Fall ist. Das gesamte Ausmaß der durch die institutionelle Trennung der beiden Hauptkompetenzen bedingten immissionsschutzrechtlichen Streitanfälligkeit des Verfahrensablaufs zeigt sich, wenn man auch die Zahl der in ein und demselben Verfahren aufgetretenen I- und NI-Streitigkeiten berücksichtigt und einander gegenüberstellt (Tab. 23).

196 Die entsprechenden Testwerte lauten im ersten Fall chi $^{2}=11,46>10,8, p=0,001$, im zweiten Fall chi ${ }^{2}=1,18<2,71$ für $p=0,1$, jeweils df $=1$. 
Tab. 23

Verfahrensbezogene Häufigkeiten immissionsschutzrechtlicher und nichtimmissionsschutzrechtlicher Einzelkontroversen zwischen den Hauptbeteiligten

\begin{tabular}{|c|c|c|c|c|c|c|}
\hline & \multicolumn{2}{|c|}{$G A A-N$} & \multicolumn{2}{|c|}{$L R A-1$} & \multicolumn{2}{|c|}{ LRA-2 } \\
\hline & I-Str. & NI-Str. & I-Str. & NI-Str. & I-Str. & NI-Str. \\
\hline Keine & $\begin{array}{c}36 \\
62,1 \%\end{array}$ & $\begin{array}{c}32 \\
55,2 \%\end{array}$ & $\begin{array}{c}12 \\
27,9 \%\end{array}$ & $\begin{array}{c}19 \\
44,2 \%\end{array}$ & $\begin{array}{c}6 \\
25,0 \%\end{array}$ & $\begin{array}{c}15 \\
62,5 \%\end{array}$ \\
\hline Eine & $\begin{array}{c}18 \\
31,0 \%\end{array}$ & $\begin{array}{c}16 \\
27,6 \%\end{array}$ & $\begin{array}{c}11 \\
25,6 \%\end{array}$ & $\begin{array}{c}15 \\
34,9 \%\end{array}$ & $\begin{array}{c}7 \\
29,2 \%\end{array}$ & $\begin{array}{c}7 \\
29,2 \%\end{array}$ \\
\hline Zwei & $\begin{array}{c}4 \\
6,9 \%\end{array}$ & $\begin{array}{c}8 \\
13,8 \%\end{array}$ & $\begin{array}{c}9 \\
20,9 \%\end{array}$ & $\begin{array}{c}7 \\
16,3 \%\end{array}$ & $\begin{array}{c}8 \\
33,3 \%\end{array}$ & $\begin{array}{c}0 \\
0,0 \%\end{array}$ \\
\hline Drei & $\begin{array}{c}0 \\
0,0 \%\end{array}$ & $\begin{array}{c}0 \\
0,0 \%\end{array}$ & $\begin{array}{c}5 \\
11,6 \%\end{array}$ & $\begin{array}{c}2 \\
4,7 \%\end{array}$ & $\begin{array}{c}2 \\
8,3 \%\end{array}$ & $\begin{array}{c}2 \\
8,3 \%\end{array}$ \\
\hline Vier & $\begin{array}{c}0 \\
0,0 \%\end{array}$ & $\begin{array}{c}2 \\
3,4 \%\end{array}$ & $\begin{array}{c}6 \\
14,0 \%\end{array}$ & $\begin{array}{c}0 \\
0,0 \%\end{array}$ & $\begin{array}{c}1 \\
4,2 \%\end{array}$ & $\begin{array}{c}0 \\
0,0 \%\end{array}$ \\
\hline Verfahren gesamt* & $\begin{array}{c}58 \\
100,0 \%\end{array}$ & $\begin{array}{c}58 \\
100,0 \%\end{array}$ & $\begin{array}{c}43 \\
100,0 \%\end{array}$ & $\begin{array}{c}43 \\
100,1 \%\end{array}$ & $\begin{array}{c}24 \\
100,0 \%\end{array}$ & $\begin{array}{c}24 \\
100,0 \%\end{array}$ \\
\hline
\end{tabular}

* Abweichungen von 100,0\% durch Rundungen bei den prozentualen Einzelhäufigkeiten.

Wie aus der Tabelle hervorgeht, finden sich beim LRA-1 in der Gruppe der Verfahren mit I-Konflikten deutlich höhere Anteile an Fällen mit zwei, drei oder vier einschlägigen Kontroversen als beim GAA-N, wo solche Fälle eher die Ausnahme darstellen. Bei den andere fachliche Belange betreffenden NI-Konflikten dagegen ergeben sich nur kleinere Differenzen in der (anteiligen) Besetzung der einzelnen Häufigkeitsklassen.

Plastischer lassen sich die dargestellten Unterschiede zwischen den beiden Modellen zum Ausdruck bringen, wenn man das Vorkommen der beiden Konfliktarten auf der Basis von Durchschnittswerten vergleicht (Tab. 23.1).

Danach hat es beim LRA-1 im Durchschnitt aller Verfahren mehr als dreimal so viele immissionsschutzrechtliche Einzelkontroversen gegeben wie beim GAA-N, nämlich rd. 1,6 gegenüber rd. 0,5. ${ }^{197}$ Die höhere Kon-

197 Die Differenz der Mittelwerte zwischen LRA-1 und GAA-N ist statistisch hochsignifikant: $\mathrm{t}=5,527>\mathrm{t}_{(0,9995 ; 99)}=3,390$, bei allerdings signifikant ungleichen Varianzen $\left(F=4,89>F_{(0,999 ; 42,57)}=2,56\right)$. - Das gleiche gilt im übrigen auch für die Differenz zwischen GAA-N und LRA-2 (Mittelwerte: $t=4,833>t_{(0,9995 ; 80)}=3,416$; Varianzen: $\left.F=3,06>F_{(0,999 ; 23,57)}=2,83\right)$. Dagegen sind die Differenzen der Werte zwischen LRA-1 und LRA-2 statistisch unerheblich (Varianzen: $F=1,60<\mathrm{F}_{(0,95}$; $42,23)=1,86$; Mittelwerte: $\left.\mathrm{t}=0,627<\mathrm{t}_{(0,95 ; 65)}=1,69\right)$. 
fliktdichte resultiert nicht allein aus einer größeren Zahl kontrovers geführter Verfahren, sondern auch aus einer größeren Zahl von Kontroversen, die im Durchschnitt auf jedes Verfahren dieser Konfliktgruppe entfallen. Beim LRA-1 sind es mit rd. 2,2 Streitigkeiten pro Verfahren fast doppelt so viele wie beim GAA-N mit rd. 1,2 Streitigkeiten pro Verfahren.

Tab. 23.1

Anzahl und statistische Parameter immissionsschutzrechtlicher und nichtimmissionsschutzrechtlicher Einzelkontroversen zwischen den Hauptbeteiligten

\begin{tabular}{|l|c|c|c|}
\cline { 2 - 4 } \multicolumn{1}{c|}{} & GAA-N & LRA-1 & LRA-2 \\
\hline Anzahl der I-Kontroversen gesamt & 26 & 68 & 33 \\
Zahl der Verfahren mit I-Streit & 22 & 31 & 18 \\
\hline I-Kontroversen pro I-Streit-Verfahren & 1,18 & 2,19 & 1,83 \\
\hline Durchschnittl. Zahl I-Kontroversen* & 0,45 & 1,58 & 1,38 \\
\hline Anzahl der NI-Kontroversen gesamt & 40 & 35 & 13 \\
Zahl der Verfahren mit NI-Streit & 26 & 24 & 9 \\
\hline NI-Kontroversen pro NI-Streit-Verfahren & 1,54 & 1,46 & 1,44 \\
\hline Durchschnittl. Zahl NI-Kontroversen* & 0,69 & 0,81 & 0,54 \\
\hline Anzahl der I-/NI-Kontroversen gesamt & 66 & 103 & 46 \\
Zahl der Verfahren mit I-/NI-Streit & 36 & 38 & 19 \\
\hline I-/NI-Kontrov. pro I-/NI-Streit-Verfahren & 1,83 & 2,71 & 2,42 \\
\hline Durchschnittl. Zahl I-/NI-Kontrov. gesamt* & 1,14 & 2,40 & 1,92 \\
\hline
\end{tabular}

* Bezogen auf die jeweiligen Grundgesamtheiten.

Ein anderes Bild ergibt sich dagegen für die nicht-immissionsschutzrechtlichen Konflikte. Hier bewegt sich die durchschnittliche Zahl einschlägiger Einzelkontroversen, bezogen auf die Gesamtheit der Verfahren, mit rd. 0,7 beim GAA-N und rd. 0,8 beim LRA-1 auf etwa gleichem Niveau. ${ }^{198}$ Auch

198 Die Differenzen der Varianzen und Mittelwerte zwischen GAA-N und LRA-1 liegen unterhalb jeglicher Signifikanz (Varianzen: $F=1,19<\mathrm{F}_{(0,95 ; 57,42)}=1,58$; Mittelwerte: $\left.\mathrm{t}=0,670<\mathrm{t}_{(0,95 ; 99)}=1,660\right)$. Das gleiche gilt für den Vergleich zwischen GAA-N und LRA-2 (Varianzen: $\mathrm{F}=1,18<\mathrm{F}_{(0,95 ; 57,23)}=1,90$; Mittelwerte: $\mathrm{t}=0,649$ $\left.<\mathrm{t}_{(0,95 ; 80)}=1,664\right)$ und zwischen LRA-1 und LRA-2 (Varianzen: $\mathrm{F}=1,01<\mathrm{F}_{(0,95 ;}$ $23,42)=1,74$; Mittelwerte: $\left.\mathrm{t}=1,214<\mathrm{t}_{(0,95 ; 65)}=1,671\right)$. 
die im Durchschnitt auf die Verfahren dieser Konfliktgruppe entfallende Zahl von Kontroversen ist mit jeweils rd. 1,5 nahezu identisch. Der Vergleich auf der Basis dieser Parameter macht nochmals deutlich, daß es die immissionsschutzrechtlichen und nicht sonstige fachliche Belange waren, die in diesem Bereich für eine mit 2,4 Kontroversen mehr als doppelt so hohe durchschnittliche Streitanfälligkeit des Verfahrensverlaufs sorgten als beim GAA-N mit 1,1 Kontroversen. ${ }^{199}$ Entsprechendes gilt für den höheren Gesamtwert von rd. 2,7 gegenüber rd. 1,8 Einzelkontroversen, mit denen die von der einen und/oder anderen Art von Konflikten betroffenen Verfahren belastet waren.

Speziell immissionsschutzrechtliche Konfliktlagen zeichneten sich im übrigen in beiden Modellen mehrheitlich bereits im Vorantragszeitraum, also vor der offiziellen Antragstellung, ab. Vor allem gingen sie signifikant häufiger als die insoweit oder gänzlich konfliktfreien Verfahren mit einer intensiveren Vorantragsberatung mit mehr als zwei Beratungskontakten einher. ${ }^{200}$ Bei den nicht-immissionsschutzrechtlichen Konflikten besteht ein vergleichbarer Zusammenhang dagegen nicht. Dies erklärt sich in erster Linie daraus, daß die sonstigen Fachbehörden nur zu einem kleineren Teil $\mathrm{zu}$ den Vorantragsberatungen beigezogen wurden (vgl. oben Ziff. 1.3.2). Mit ihnen konnten sich folglich Konflikte großteils erst nach ihrer offiziellen Verfahrensbeteiligung ergeben.

Die Möglichkeit, daß das ermittelte Nord-Süd-Gefälle bei den immissionsschutzrechtlichen Streitigkeiten modellunabhängigen Randbedingungen zuzuschreiben ist, wurde im Hinblick auf die Unternehmensgröße, die Genehmigungsart und die Anlagenart (gem. Anhang der 4. BImSchV) überprüft. In beiden Modellen hat sich bei keinem der drei Faktoren ein statistischer Zusammenhang mit der Häufigkeit von I-Konflikten ergeben. ${ }^{201}$

199 Die Differenz der Mittelwerte zwischen LRA-1 und GAA-N ist statistisch hochsignifikant: $\mathrm{t}=4,360>\mathrm{t}_{(0,9995 ; 99)}=3,390$, bei statistisch gleichen Varianzen $(\mathrm{F}=1,78$ $\left.<\mathrm{F}_{(0,99 ; 42,57)}=1,88\right)$. - Das gleiche gilt für die Mittelwertunterschiede zwischen LRA-2 und GAA-N, wenn auch hier auf etwas niedrigerem Signifikanzniveau (Mittelwerte: $\mathrm{t}=2,370>\mathrm{t}_{(0,975 ; 80)}=1,990$, bei gleichen Varianzen: $\mathrm{F}=1,61<\mathrm{F}_{(0,95 ; 23}$, $\left.{ }_{57}=1,74\right)$. Dagegen sind die Differenzen der Werte zwischen LRA-1 und LRA-2 statistisch unerheblich (Mittelwerte: $\mathrm{t}=1,146<\mathrm{t}_{(0,95 ; 65)}=1,69$, bei gleichen Varianzen: $\left.\mathrm{F}=1,10<\mathrm{F}_{(0,95 ; 42,23)}=1,86\right)$.

200 Vgl. zu den Häufigkeiten der Kreuztabellierung im einzelnen Tab. 23a-A im Anhang. Die angesprochene Differenz ist beim GAA-N wie beim LRA-1 signifikant $\left(\mathrm{chi}^{2}=4,16>3,84, \mathrm{p}=0,05\right.$ bzw. $\mathrm{chi}^{2}=2,72>2,71, \mathrm{p}=0,1$, jeweils df $\left.=1\right)$. Das niedrige Signifikanzniveau beim LRA-1 hängt damit zusammen, daß dort die Verfahren insgesamt beratungsintensiver geführt wurden als beim GAA-N (vgl. oben Ziff. 1.3.1).

201 Vgl. zu den Häufigkeiten der Kreuztabellierungen im einzelnen Tab. 23b-A (Unternehmensgröße), Tab. 23c-A (Genehmigungsart) u. Tab. 23d-A (Anlagenart) jeweils im Anhang. 


\subsubsection{Gegenstände und Ausgang immissionsschutzrechtlicher}

Kontroversen

Bei den immissionsschutzrechtlichen Kontroversen lassen sich nach den Konfliktgegenständen zwei Grundarten unterscheiden: Solche, die fachtechnische bzw. fachspezifische Anforderungen an Anlagenausstattung und/oder Anlagenbetrieb betreffen, und solche, bei denen Fragen der verfahrensrechtlichen Durchführung zur Debatte stehen. Fachspezifische Kontroversen umfassen dabei im einzelnen Auseinandersetzungen um (gutachtliche) Emissions- bzw. Immissionsprognosen und Meßnachweise einschließlich Meßvorschriften, um Sanierungserfordernisse und Sanierungsfristen, um bauliche Umweltschutzmaßnahmen und nicht zuletzt um Grenzwerte für Emissionen bzw. Immissionen. Den verfahrensrechtlichen Kontroversen sind im einzelnen Dispute um die grundsätzliche Genehmigungsbedürftigkeit einer Anlage oder deren (als »wesentlich« zu betrachtende) Änderung, um die der Genehmigungspflicht im einzelnen unterfallenden Anlagenteile, darunter insbesondere sog. Nebeneinrichtungen, ${ }^{202}$ um Zulassung vorzeitigen Beginns (gem. § 15a BImSchG) ${ }^{203}$, um (vom Antragsteller ergänzend zu beantragende) Befristungen der Genehmigung und schließlich auch um formgerechte Antragsgestaltung (etwa die Verwendung der behördlichen Antragsformulare) zugeordnet. In diese Rubrik fallen des weiteren Auseinandersetzungen über angedrohten Verwaltungszwang wie insbesondere Stillegungsverfügungen wegen ungenehmigten Betriebs und nachträgliche Anordnungen zur Durchsetzung von Sanierungsforderungen. Im Süden kommen noch streitige Verhandlungen um den Abschluß bzw. die Ausgestaltung eines öffentlich-rechtlichen Vertrags als Alternative zur nachträglichen Anordnung und einer ggf. erforderlichen Änderungsgenehmigung hinzu. ${ }^{204}$

Konfliktbeteiligte sind in beiden Untergruppen Antragsteller und Genehmigungsbehörde. Im Süden kommt als weitere Konfliktlinie die zwischen LRA und zugehörigem GAA in Frage, die entlang der ihnen jeweils institutionell zugewiesenen genuinen Kompetenzbereiche verläuft. Dabei inter-

202 Zur Legaldefinition der Nebeneinrichtung vgl. § 1 Abs. 2 der 4. BImSchV. In der Anwendungspraxis bereitet dieser Begriff nicht weniger Abgrenzungsprobleme als die im Gesetz nicht näher definierte »wesentliche« Änderungsgenehmigung.

203 Zur Häufigkeit entsprechender Anträge vgl. oben Anm. 50.

204 Das Instrument des öffentlich-rechtlichen Vertrags kam nur beim LRA-1 zur Anwendung. Inzwischen hat man dort jedoch davon Abstand genommen, weil die meisten Betriebe einen Vertragsabschluß verweigerten. Solche freiwilligen Selbstbindungen waren am ehesten große Firmen eingegangen, die über die notwendige Verhandlungsmacht verfügten, günstige Vertragsbedingungen für sich auszuhandeln. $\mathrm{Zu}$ den Einsatzmöglichkeiten des öffentlich-rechtlichen Vertrags als alternativer Handlungsmöglichkeit der Verwaltung vgl. den Erfahrungsbericht von Arnold 1989. 
essiert hier auch die Frage, wieweit es in der Verfahrenspraxis zu einseitigen bzw. wechselseitigen Kompetenzüberschreitungen gekommen ist.

Tab. 24 zeigt die Häufigkeiten der Verfahren mit Kontroversen der einen oder anderen Art. Da in einigen Verfahren sowohl fachspezifische als auch verfahrensrechtliche Auseinandersetzungen aufgetreten sind, liegt die Summe der Fälle beider Varianten über der Gesamtzahl der mit I-Konflikten einhergegangenen Verfahren. Außerdem gibt die Tabelle an, wieviel Einzelkontroversen sich insgesamt und im Durchschnitt je Konfliktfall in jeder der beiden Gruppen abgespielt haben (Zeilen im Kleindruck).

Wie bereits erwähnt, gingen insgesamt fast doppelt so viele Verfahren beim LRA-1 wie beim GAA-N, nämlich gut zwei Drittel gegenüber gut einem Drittel, mit immissionsschutzrechtlichen Streitigkeiten einher. Insofern kann es nicht überraschen, daß sich diese Unterschiede in der Grundtendenz auch in jeder der beiden Untergruppen von Kontroversen wiederfinden. Daß sie so hoch ausfallen - mit einer Differenz von 36,2 Prozentpunkten bei den fachspezifischen bzw. von 33,5 Prozentpunkten bei den verfahrensrechtlichen Streitigkeiten ${ }^{205}$-, war dagegen weniger zu erwarten. Die höhere Streitwilligkeit (in immissionsschutzbezogenen Fragen) beim LRA-1 gegenüber dem GAA-N spiegelt sich auch in der Zahl der im Durchschnitt auf jeden Konfliktfall entfallenden Einzelkontroversen wider. Bei den Verfahren mit fachlichen Divergenzen kommen auf jedes der einschlägigen Verfahren 1,3 Einzelkontroversen beim LRA-1 gegenüber 1,0 beim GAA-N, bei denen mit verfahrensrechtlichen Streitigkeiten 1,56 Einzelkontroversen im Süden gegenüber 1,18 im Norden.

Darüber hinaus fällt auf, daß bei beiden Modellen die verfahrensrechtlichen Kontroversen jeweils die fachspezifischen überwiegen. Während beim LRA-1 die Abweichung von der theoretisch zu erwartenden Gleichverteilung $^{206}$ aber noch innerhalb der statistischen Toleranz liegt, ${ }^{207}$ sind beim GAA-N die fachlichen Kontroversen im Verhältnis zu den verfahrensrechtlichen signifikant unterrepräsentiert. ${ }^{208}$

205 In beiden Fällen sind die Unterschiede hochsignifikant (fachliche Kontroversen: chi $^{2}=16,77$, verfahrensrechtliche Kontroversen: chi $^{2}=13,27$, jeweils $>10,8, p=$ $0,001, \mathrm{df}=1$ ).

206 Es ist kein theoretischer Grund - die pseudo-theoretische Figur der »Natur der Sache« eingeschlossen - ersichtlich, etwas anderes zu erwarten und damit eine den Verfahren innewohnende Tendenz, Konflikte der einen oder anderen Art vermehrt zu provozieren, zu unterstellen. Wenn es eines empirischen Belegs bedürfte, wäre er - im Vorgriff auf spätere Befunde - bei den nicht-immissionsschutzrechtlichen Konflikten zu finden, bei denen in beiden Genehmigungsmodellen die empirische Verteilung der beiden Arten von Kontroversen nicht signifikant von der theoretisch $\mathrm{zu}$ erwartenden Gleichverteilung abweicht (mit den Testwerten: chi $^{2}=0,50$ beim GAA-N und $\mathrm{chi}^{2}=1,58$ beim LRA-1, jeweils $<2,71$ für $\mathrm{p}=0,1$ und $\mathrm{df}=1$ ). $\mathrm{S}$. dazu im einzelnen nachfolgende Ziff. 3.1.3.

$207 \mathrm{chi}^{2}=1,04<2,71$ für $\mathrm{p}=0,1$ und $\mathrm{df}=1$.

$208 \mathrm{chi}^{2}=5,26>5,02, \mathrm{p}=0,025, \mathrm{df}=1$. 
Tab. 24

Gegenstände der Kontroversen in den Verfahren mit immissionsschutzrechtlichen (I-)Konflikten (Häufigkeiten)

\begin{tabular}{|l|c|c|c|}
\cline { 2 - 4 } \multicolumn{1}{c|}{} & GAA-N & $L R A-1$ & LRA-2 \\
\hline a) Verfahren mit fachlich-technischen & 6 & 20 & 4 \\
Kontroversen & $10,3 \%$ & $46,5 \%$ & $16,7 \%$ \\
[Zahl der Einzelkontroversen] & {$[6]$} & {$[26]$} & {$[4]$} \\
[Einzelkontrov. pro Verfahren] & {$[1,0]$} & {$[1,3]$} & {$[1,0]$} \\
\hline b) Verfahren mit verfahrensrechtlichen & 17 & 27 & 18 \\
Kontroversen & $29,3 \%$ & $62,8 \%$ & $75,0 \%$ \\
[Zahl der Einzelkontroversen] & {$[20]$} & {$[42]$} & {$[29]$} \\
[Einzelkontrov. pro Verfahren] & {$[1,18]$} & {$[1,56]$} & {$[1,61]$} \\
\hline Verfahren mit I-Konflikten gesamt* & 22 & 31 & 18 \\
\hline Verfahren ohne I-Konflikte & $37,9 \%$ & $72,1 \%$ & $75,0 \%$ \\
\hline \hline Verfahren gesamt & 36 & 12 & 6 \\
\hline
\end{tabular}

* Da sich die beiden Unterkategorien (a und b) nicht wechselseitig ausschließen, liegt die Summe der absoluten und relativen Häufigkeiten über der Gesamtzahl der absoluten bzw. relativen Häufigkeiten der Gesamtzahl der Verfahren mit I-Konflikten.

Auch für das Verhältnis zwischen immissionsschutzrechtlichen und nichtimmissionsschutzrechtlichen Konflikten rechtfertigt sich die theoretische Erwartung einer Gleichverteilung. Der Vergleich mit den vorgefundenen empirischen Verteilungen ergibt dabei folgende Gewichtungen: Bei den fachlichen Kontroversen sind die I-Konflikte gegenüber den NI-Konflikten beim GAA-N signifikant unterrepräsentiert, beim LRA-1 entfallen auf beide Konfliktgruppen (statistisch) gleiche Anteile. ${ }^{209}$ Bei den verfahrensrechtlichen Kontroversen hingegen sind die I-Konflikte gegenüber den NIKonflikten beim LRA-1 signifikant überrepräsentiert, beim GAA-N entfallen auf beide Konfliktgruppen (statistisch) gleiche Anteile. ${ }^{210}$

Welche Schlußfolgerungen diese Zusammenhänge für die jeweilige Position des GAA in den beiden Genehmigungsmodellen nahelegen, wird abschließend zu erörtern sein, um auch die nachfolgenden Befunde zum Aus-

209 Die entsprechenden Testwerte lauten: chi $^{2}=6,00>5,02, p=0,025$ beim GAA-N und $\mathrm{chi}^{2}=0,05<2,71$ für $\mathrm{p}=0,1$ beim LRA-1, jeweils $\mathrm{df}=1$.

210 Die entsprechenden Testwerte lauten: $\operatorname{chi}^{2}=7,41>6,64, p=0,001$ beim LRA-1 und $\mathrm{chi}^{2}=0,29<2,71$ für $\mathrm{p}=0,1$ beim GAA-N, jeweils $\mathrm{df}=1$. 
gang der verschiedenen Arten von Kontroversen und Konflikten berücksichtigen zu können.

Die Ergebnisse dazu sind, getrennt nach den beiden Arten von Kontroversen, in Tab. 24.1 zusammengestellt. Innerhalb jeder Gruppe sind die einzelnen Streitfälle unter dem Gesichtspunkt bilanziert, wieweit ihr jeweiliger Ausgang eher den Interessen des Betreibers entgegenkam oder zuwiderlief, sie also letztlich eher zu dessen Gunsten oder eher zu dessen Lasten entschieden wurden. Für den Süden ist zusätzlich berücksichtigt, wieweit sich das GAA in umstrittenen fachtechnischen Fragen bzw. das LRA in umstrittenen verfahrensrechtlichen Fragen durch Optionen oder »Einmischungen« des jeweils anderen Amts in seiner Entscheidung hat beeinflussen lassen (Zeilen im Kleindruck).

Tab. 24.1

Ausgang der immissionsschutzrechtlichen Einzelkontroversen

\begin{tabular}{|l|c|c|c|}
\cline { 2 - 4 } \multicolumn{1}{c|}{ a) Fachliche Kontroversen } & GAA-N & LRA-1 & LRA-2 \\
\hline GAA setzt sich durch & 3 & 15 & 1 \\
zu Lasten des Betreibers & $11,5 \%$ & $22,1 \%$ & $3,0 \%$ \\
[davon: gegen Position des LRA-1/-2] & - & {$[4]$} & {$[0]$} \\
\hline GAA gibt nach & 3 & 11 & 3 \\
zugunsten des Betreibers & $11,5 \%$ & $16,2 \%$ & $9,1 \%$ \\
[davon: bei Fürsprache seitens LRA-1/-2 & - & {$[7]$} & {$[2]$} \\
\hline
\end{tabular}

b) Verfahrensrechtliche Kontroversen

\begin{tabular}{|l|c|c|c|}
\hline Genehmigungsbehörde setzt sich durch & 8 & 24 & 12 \\
Zu Lasten des Betreibers & $30,8 \%$ & $35,3 \%$ & $36,4 \%$ \\
[davon: in Abstimmung mit GAA-1/-2] & - & {$[9]$} & {$[7]$} \\
[davon: auf Druck von GAA-1/-2] & - & {$[13]$} & {$[0]$} \\
[davon: gegen Position von GAA-1/-2] & - & {$[2]$} & {$[3]$} \\
\hline Genehmigungsbehörde gibt nach & 12 & 18 & 17 \\
zugunsten des Betreibers & $46,2 \%$ & $26,5 \%$ & $51,5 \%$ \\
[davon: in Abstimmung mit GAA-1/-2] & - & {$[7]$} & {$[11]$} \\
[davon: gegen Position von GAA-1/-2] & - & {$[11]$} & {$[3]$} \\
\hline \hline I-Einzelkontroversen gesamt* & 26 & 68 & 33 \\
\hline
\end{tabular}

* Abweichungen von 100,0\% durch Rundungen bei den prozentualen Einzelhäufigkeiten. 
Richtet man zunächst den Blick auf die (kleinere) Gruppe der fachlichen Kontroversen, in denen die Kompetenz des GAA als zuständiger Fachbehörde gefragt ist, ergibt sich folgendes Bild. Das GAA-N hielt hier je zur Hälfte der (wenigen) Streitigkeiten an seinen Forderungen gegen den Betreiber fest bzw. gab zu dessen Gunsten nach. Das baden-württembergische GAA-1 setzte sich in den (erheblich zahlreicheren) Streitfällen etwas häufiger mit seinen Anforderungen durch - in vier Fällen gegen das ausdrückliche, zugunsten des Betreibers plädierende Votum des LRA-1 -, als daß es sich zu Zugeständnissen bereit erklärte. Das Entgegenkommen war zum (kleineren) Teil eine Reaktion auf die Kooperationsbereitschaft des Betreibers, seinerseits Vorleistungen zu erbringen, zum (größeren) Teil eine Reaktion auf einen »starken« Kontrahenten. Dessen Stärke beruhte entweder (in vier Fällen) auf einer Kombination von einsetzbarer Beschwerdemacht als Großunternehmen und (wirtschaftspolitisch) motivierter Unterstützung durch das LRA-1 oder (in drei Fällen) darauf, daß der Betreiber den Dezernenten des LRA-1 in das Verfahren einschaltete. Sieht man von der letztgenannten, spezifischen Konstellation einmal ab, ergibt sich, daß das GAA1 in den (acht) Fällen, in denen sich das LRA-1 für den Betreiber verwandt hatte, jeweils zur Hälfte dem »Druck « widerstand bzw. nachgab. In diesen »hart« umstrittenen Fällen weist das GAA-1 demnach eine ausgeglichene Bilanz in seinem Durchsetzungsvermögen gegenüber dem LRA-1 auf. In allen übrigen Fällen traf es seine Entscheidungen völlig autonom, abgesehen von den drei Fällen, in denen es sich von einer höheren Hierarchieebene hat beeindrucken lassen, wenn dieser auch keine formelle Weisungsbefugnis zusteht. ${ }^{211}$

Bei den verfahrensrechtlichen Kontroversen, die gegenüber den fachlichen Kontroversen häufiger aufgetreten sind, ist der formellen Kompetenz nach in erster Linie die Genehmigungsbehörde angesprochen. Das auch diese Funktion wahrnehmende GAA-N entschied die Streitfälle etwas häufiger zugunsten als zu Lasten des Betreibers (in der Relation $12 \mathrm{zu} 8$ ), beim LRA-1 verhält es sich umgekehrt (in der Relation $18 \mathrm{zu} 24$ ). Der Unterschied ist allerdings nicht signifikant. ${ }^{212}$ Die Streitigkeiten wären beim LRA-1 jedoch noch häufiger zugunsten des Betreibers ausgegangen, wenn dieses sich nicht mehrfach (in 13 Fällen) gezwungen gesehen hätte, sich dem auf Durchsetzung bestimmter verfahrensrechtlicher Positionen insistierenden GAA-1 zu beugen. Von mangelndem Vertrauen in die eigene Kompetenz zeugen auch die (insgesamt 16) Fälle, in denen das LRA-1 seine - zugunsten oder auch zu Lasten des Betreibers - geplante Entscheidung dem GAA-1 gleichsam zur Billigung vorlegte. Den genannten 13

211 Informell verfügt sie gleichwohl über die Möglichkeit, die Vorgesetztenebene des GAA-1 einzuschalten, dem es insofern geraten erscheint, solchen Konflikten lieber durch Nachgeben auszuweichen.

$212 \mathrm{chi}^{2}=1,59<2,71$ für $\mathrm{p}=0,1$ und $\mathrm{df}=1$. 
Streitfällen, in denen die beiden Behörden gegensätzliche Positionen vertraten und in denen das GAA-1 mit seiner Fachkompetenz »obsiegte «, stehen 11 Fälle gegenüber, in denen das LRA-1 den Dissens autoritativ mit Hilfe seiner formellen Entscheidungsmacht in seinem Sinne »auflöste«. Rein quantitativ betrachtet, ist die Bilanz zwischen den »Kontrahenten « nahezu ausgeglichen. Von der Sache her gewann das GAA-1 vor allem in Fragen der grundsätzlichen Genehmigungsbedürftigkeit, der Genehmigungsart und der im einzelnen genehmigungspflichtigen Anlagenteile und Nebeneinrichtungen sowie der Durchsetzung von Sanierungserfordernissen die Oberhand. Demgegenüber spielte das LRA-1 seine Genehmigungskompetenz vor allem dort (und zugunsten des Betreibers) aus, wo es ihm abverlangte Verwaltungszwangsmaßnahmen wie Stillegungsverfügungen und Bußgeldverhängung verweigerte oder auf eine formgerechte Antragsausarbeitung verzichtete. Zugespitzt gesagt, machte das LRA-1 hauptsächlich in den Materien von seiner formellen Entscheidungsmacht Gebrauch, deren Beurteilung am wenigsten fachtechnischen Sachverstand voraussetzte.

Sieht man einmal vom Ausgang der verfahrensrechtlichen Kontroversen für den Betreiber ab und betrachtet sie allein mit Blick auf das interne Kräfteverhältnis zwischen den beiden Behörden, erweist sich das GAA-1 eindeutig als die dominierende Instanz. Es kann zu seinen Gunsten insgesamt 29 Fälle verbuchen, in denen es sich entweder gegen die Position des LRA1 durchsetzte (13) oder in denen die Entscheidung ausdrücklich von seinem Votum abhängig war (16). Dem stehen lediglich 13 Fälle gegenüber, in denen sich das LRA-1 behaupten konnte. Die Differenz ist statistisch signifikant. $^{213}$

\subsubsection{Gegenstände und Ausgang nicht-immissionsschutzrechtlicher Kontroversen}

Analog zu den immissionsschutzrechtlichen Streitfällen lassen sich auch die nicht-immissionsschutzrechtlichen Konflikte nach den beiden Grundarten fachspezifischer und verfahrensrechtlicher Kontroversen aufschlüsseln. Konfliktbeteiligte sind hier die jeweilige (sonstige) Fachbehörde, die Genehmigungsbehörde und, unmittelbar oder mittelbar, der Antragsteller. ${ }^{214}$

Bei den konfliktbeteiligten Fachbehörden handelte es sich vor allem um die Fachämter für Bauwesen, Wasser- und Bodenschutz sowie für den Naturschutz. Letzteres zeichnete sich beim LRA-1 - im Unterschied zum GAA-N - durch eine im Verhältnis zu seiner Beteiligungsquote an den Ver-

$213 \mathrm{chi}^{2}=6,10>5,02, \mathrm{p}=0,025, \mathrm{df}=1$.

214 In einigen wenigen Fällen war auch das GAA-1 mittelbar beteiligt. Wegen ihres Ausnahmecharakters wird diese Konstellation im folgenden nicht gesondert berücksichtigt. 
fahren (vgl. oben Ziff 2.3.2, Tab. 14) überproportionale Präsenz aus. Den Fachbehörden zugerechnet wurden außerdem die Gemeinden in ihrer Eigenschaft als planungsrechtliche Hoheitsheitsträger. ${ }^{215}$

Unter die fachlichen Kontroversen fallen auch hier Auseinandersetzungen um spezifische Anforderungen an Anlagenausstattung und/oder Anlagenbetrieb. Die verfahrensrechtlichen Kontroversen betrafen hauptsächlich Divergenzen über fachbehördliche Beteiligungserfordernisse und Zuständigkeiten für den jeweils in Frage stehenden Belang sowie im Einzelfall auch Verhandlungen über eine informelle »Arbeitsteilung « zwischen Fachund Genehmigungsbehörde, etwa durch die separate Erteilung der erforderlichen Baugenehmigung durch das Bauamt. ${ }^{216}$ Hinzu kommen Streitigkeiten um - aus Sicht der Genehmigungsbehörde - Überschreitungen der fachbehördlichen Kompetenzen durch ihnen nicht zustehende, eigenmächtige Anordnungen und Verfügungen gegen den Antragsteller und (in umgekehrter Richtung) Forderungen der Fachbehörden an die Genehmigungsbe-hörde, ihren Belangen durch Zwangsmaßnahmen gegen den Betreiber zur Durchsetzung zu verhelfen.

Tab. 25 gibt die Häufigkeiten wieder, mit denen jede der beiden Arten von Kontroversen aufgetreten ist. Auch hier übersteigt die Summe der Fälle beider Varianten die Gesamtzahl der mit NI-Konflikten einhergegangenen Verfahren, weil einige von ihnen sowohl in fachlicher als auch in verfahrensrechtlicher Hinsicht kontrovers verlaufen sind. Analog zu Tab. 24 führt auch die vorliegende Tabelle für jede der beiden Gruppen die Gesamtzahl der Einzelkontroversen und die Anzahl pro Konfliktfall auf (Zeilen im Kleindruck).

Beim GAA-N wie beim LRA-1 wurde gleichermaßen in jeweils rd. einem Viertel der Verfahren $(24,1 \%$ bzw. $27,9 \%)$ um verfahrensrechtliche Fragen im Zusammenhang mit nicht-immissionsschutzrechtlichen Belangen gestritten. Häufiger kam es, wie schon angedeutet (vgl. oben Ziff. 3.1.2), zu fachlichen Kontroversen mit Anteilen von 44,2\% beim LRA-1 bzw. von $31,0 \%$ beim GAA-N, ${ }^{217}$ wobei diese Differenz statistisch nicht

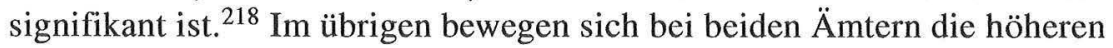

215 Auf mitunter von den Gemeinden unternommene Versuche, anläßlich ihrer Verfahrensbeteiligung auch durch ihre planungsrechtlichen Befugnisse nicht gedeckte Interessen zu verfolgen, wurde bereits hingewiesen (s.o. Anm. 135). Ihre Präsenz mündete aber nicht häufiger als bei den sonstigen Fachbehörden in Konflikte, selbst im Süden mit seiner großzügigen Beteiligungspraxis.

216 Mit dem Ziel einer Beschleunigung des Verfahrens, obwohl dies gerade einer der Hauptgründe für die Einführung des Konzentrationsprinzips war.

217 Das LRA-2, das sich mit einem Anteil von 25,0\% an verfahrensrechtlichen Kontroversen auf dem Niveau der anderen beiden Genehmigungsbehörden bewegt, liegt bei den fachlichen Kontroversen mit einem Anteil von nur 16,7\% deutlich unter deren Werten.

$218 \mathrm{chi}^{2}=1,84<2,71$ für $\mathrm{p}=0,1$ und $\mathrm{df}=1$. 
Anteile an Verfahren mit fachlichen Konflikten gegenüber denen mit verfahrensrechtlichen Streitigkeiten noch innerhalb der statistischen Toleranzgrenzen einer theoretisch zu erwartenden Gleichverteilung. ${ }^{219}$

Tab. 25

Gegenstände der Kontroversen in den Verfahren mit nicht-immissionsschutzrechtlichen (NI-)Konflikten (Häufigkeiten)

\begin{tabular}{|l|c|c|c|}
\cline { 2 - 4 } \multicolumn{1}{c|}{} & GAA-N & LRA-1 & LRA-2 \\
\hline a) Verfahren mit fachlich-technischen & 18 & 19 & 4 \\
Kontroversen & $31,0 \%$ & $44,2 \%$ & $16,7 \%$ \\
[Zahl der Einzelkontroversen] & {$[24]$} & {$[21]$} & {$[5]$} \\
[Einzelkontrov. pro Verfahren] & {$[1,33]$} & {$[1,11]$} & {$[1,25]$} \\
\hline b) Verfahren mit verfahrensrechtlichen & 14 & 12 & 6 \\
Kontroversen & $24,1 \%$ & $27,9 \%$ & $25,0 \%$ \\
[Zahl der Einzelkontroversen] & {$[16]$} & {$[14]$} & {$[10]$} \\
[Einzelkontrov. pro Verfahren] & {$[1,14]$} & {$[1,17]$} & {$[1,67]$} \\
\hline \hline Verfahren mit NI-Konflikten gesamt* & 26 & 24 & 9 \\
\hline Verfahren ohne NI-Konflikte & $44,8 \%$ & $55,8 \%$ & $37,5 \%$ \\
\hline \hline Verfahren gesamt & 32 & 19 & 15 \\
\hline
\end{tabular}

* Da sich die beiden Unterkategorien (a und b) nicht wechselseitig ausschließen, liegt die Summe der absoluten und relativen Häufigkeiten über der absoluten bzw. relativen Gesamtzahl der Verfahren mit I-Konflikten.

Auch bei der Zahl von Einzelkontroversen, die im Durchschnitt auf jeden NI-Konfliktfall in beiden Streitkategorien entfallen (Zeilen im Kleindruck), differieren die beiden Genehmigungsmodelle in geringerem Maße, als dies bei den I-Konflikten zu beobachten war. Bei den Verfahren mit verfahrensrechtlichen Divergenzen kommen auf jedes der einschlägigen Verfahren 1,17 Einzelkontroversen beim LRA-1 gegenüber 1,14 beim GAA-N. Bei den Verfahren mit fachlichen Streitigkeiten liegt der Durchschnittswert von 1,33 Einzelkontroversen im Norden zwar über dem entsprechenden Wert von 1,11 im Süden, in dieser Relation läßt sich jedoch ein gewisser Ausgleich für den erwähnten niedrigeren Anteil an Verfahren mit fachlichen Konflikten beim GAA-N gegenüber dem beim LRA-1 sehen. 
Nicht nur in quantitativer Hinsicht, sondern auch im Hinblick auf den jeweiligen Ausgang der Einzelkontroversen zeigen sich weitgehende Affinitäten zwischen den beiden Modellen. Die Ergebnisse sind in Tab. 25.1, getrennt nach Konfliktart, dargestellt. Bei der Bilanzierung von »Gewinnen « und »Verlusten « ist zu berücksichtigen, daß sich die fachlichen Kontroversen in erster Linie zwischen der jeweiligen Fachbehörde und dem Antragsteller abspielen. Die Position des Fachamts ist von daher vergleichbar derjenigen des GAA bei den immissionsschutzbezogenen Auseinandersetzungen. Die Genehmigungsbehörde kann sich auch hier zugunsten der einen oder anderen Seite verwenden (Zeilen im Kleindruck). Demgegenüber beziehen sich die verfahrensrechtlichen Kontroversen in erster Linie auf das Binnenverhältnis zwischen Fachbehörde und Genehmigungsbehörde. Insofern betreffen sie nur mittelbar den Antragsteller, was allerdings nicht ausschließt, daß der Streitausgang im Ergebnis seinen Interessen ebenso entgegenkommen wie zuwiderlaufen kann (Zeilen im Kleindruck). Die Befunde, die sich allerdings nur auf niedrige (absolute) Fallzahlen stützen können, weisen für beide Konfliktarten weitgehend ausgeglichene »Gewinn-Verlust«-Bilanzen im Norden wie im Süden aus. Bei den fachlichen Kontroversen setzte sich die Fachbehörde (nahezu) ebenso häufig gegenüber den Wünschen des (künftigen) Betreibers durch wie sie diesen nachgab, beim GAA-N in der Relation von jeweils 12 Fällen (absolut), beim LRA-1 in der Relation von 9 zu 12 Fällen (absolut). Ähnlich ausgewogen ist die Bilanz bei den verfahrensrechtlichen Kontroversen. Beim GAA-N »obsiegte « die Genehmigungsbehörde in 9 Fällen, in 7 Fällen steckte sie gegenüber der Fachbehörde zurück. Beim LRA-1 verbucht die Genehmigungsbehörde bei einer Relation von 9 zu 5 Fällen etwas mehr »Gewinne«, die Differenz liegt aber weit unterhalb jeglicher statistischer Signifikanz.

Tab. 25.1

Ausgang der nicht-immissionsschutzrechtlichen Einzelkontroversen

a) Fachliche Kontroversen

\begin{tabular}{|l|c|c|c|}
\cline { 2 - 4 } \multicolumn{1}{c|}{} & $G A A-N$ & $L R A-1$ & $L R A-2 *$ \\
\hline Fachbehörde setzt sich durch & 12 & 9 & 2 \\
zu Lasten des Betreibers & $30,0 \%$ & $25,7 \%$ & $*$ \\
[davon: mit Unterstützung der Genehm.Beh.] & {$[4]$} & {$[1]$} & {$[0]$} \\
[davon: gegen Position der Genehm.Beh.] & {$[3]$} & {$[0]$} & {$[0]$} \\
\hline Fachbehörde gibt nach & 12 & 12 & 3 \\
zugunsten des Betreibers & $30,0 \%$ & $34,3 \%$ & $*$ \\
[davon: mit Unterstützung der Genehm.Beh.] & {$[9]$} & {$[7]$} & {$[2]$} \\
[davon: gegen Position der Genehm.Beh.] & {$[0]$} & {$[1]$} & {$[0]$} \\
\hline
\end{tabular}




\begin{tabular}{|l|c|c|c|}
\hline Genehmigungsbehörde setzt sich & 9 & 9 & 6 \\
gegen Fachbehörde durch & $22,5 \%$ & $25,7 \%$ & $*$ \\
[davon: zugunsten des Betreibers] & {$[7]$} & {$[7]$} & {$[4]$} \\
[davon: zu Lasten des Betreibers] & {$[1]$} & {$[2]$} & {$[0]$} \\
\hline Genehmigungsbehörde gibt nach & 7 & 5 & 2 \\
gegenüber Fachbehörde & $17,5 \%$ & $14,3 \%$ & $*$ \\
[davon: zugunsten des Betreibers] & {$[0]$} & {$[2]$} & {$[0]$} \\
[davon: zu Lasten des Betreibers] & {$[2]$} & {$[1]$} & {$[0]$} \\
\hline \hline & 40 & 35 & 13 \\
NI-Einzelkontroversen gesamt & $100,0 \%$ & $100,0 \%$ & $*$ \\
\hline
\end{tabular}

* Keine Prozentuierung wegen zu kleiner Gesamtfallzahl.

Soweit die Genehmigungsbehörde bei den (fachlichen) Kontroversen zwischen Fachbehörde und Betreiber intervenierte, geschah dies in beiden Modellen in der Mehrheit der Fälle zugunsten des Betreibers und nur in der Minderheit zu dessen Lasten. Beim GAA-N beläuft sich die Relation auf 12 zu 4, beim LRA-1 auf 7 zu 2 Fälle. Zusammengenommen, d. h. unabhängig von der jeweils gegenüber den Betreiberinteressen eingenommenen Position, engagierte sich die niedersächsische Genehmigungsbehörde in 16 der fraglichen 24 Fälle und damit deutlich häufiger als die baden-württembergische, die dies in 9 der 21 einschlägigen Fälle tat. Die Differenz unterschreitet allerdings, wenngleich nur knapp, die statistische Signifikanzgrenze. ${ }^{220}$ Immerhin deuten die Befunde darauf hin, daß das technisch versierte Personal der niedersächsischen Gewerbeaufsicht sich eher ein kompetentes Mitreden bei den überwiegend ebenfalls technikbezogenen Belangen anderer Behörden zutraut, als dies bei den baden-württembergischen Verwaltungsfachleuten der Fall ist.

Die Interventionsneigung des LRA-1 in den vorliegenden nicht-immissionsschutzbezogenen fachlichen Streitfällen differiert im Ergebnis nicht von derjenigen, die es in den immissionsschutzbezogenen Fachkonflikten gezeigt hat. ${ }^{221}$ M.a.W. es sieht für sich gegenüber dem GAA keine größeren Chancen, auf dessen fachspezifische Entscheidungen Einfluß zu nehmen, als gegenüber den übrigen Fachbehörden, obwohl es zu jenem die engeren, kontinuierlicheren Beziehungen unterhält und dabei auch eine gewisse Vertrautheit mit technischen Fragen und Lösungen erworben haben dürfte. Ein solches formell nicht zertifiziertes (lediglich »privates«) Wissen scheint

$220 \mathrm{chi}^{2}=2,58<2,71$ für $\mathrm{p}=0,1$ und $\mathrm{df}=1$.

221 Beim GAA-N entfällt ein solcher Vergleich, da es Fach- und Genehmigungskompetenz in sich vereinigt. 
aber offenbar nur bedingt, in Konfliktfällen zumal, den Handlungsspielraum der Verwaltung auf diesem Sektor zu erweitern.

\subsubsection{Einschaltung vorgesetzter Behörden}

Ein weiterer Unterschied zwischen den beiden Modellen stellt sich heraus, wenn man die jeweilige Bereitschaft der Genehmigungsbehörde, sich zur Lösung von Konflikten an eine vorgesetzte Behörde zu wenden, vergleicht. ${ }^{222}$ Tab. 26 gibt einen Überblick über Häufigkeit und Anlässe von Auskunftsersuchen an übergeordnete Stellen. ${ }^{223}$ In der Regel handelt es sich dabei um die Mittelinstanz (Bezirksregierung bzw. Regierungspräsidium), lediglich in einem Fall um das Umweltministerium.

Tab. 26

Einschaltung vorgesetzter Behörden

\begin{tabular}{|l|c|c|c|}
\cline { 2 - 4 } \multicolumn{1}{c|}{} & GAA-N & LRA-1 & LRA-2 $*$ \\
\hline Einschaltung vorgesetzter Behörden & 7 & 10 & 3 \\
& $12,1 \%$ & $23,3 \%$ & $12,5 \%$ \\
[a) Anläßlich von I-Kontroversen] & {$[1]$} & {$[8]$} & {$[2]$} \\
[b) Anläßlich von NI-Kontroversen] & {$[3]$} & {$[0]$} & {$[0]$} \\
[c) Zur Klärung von I-Fragen] & {$[1]$} & {$[1]$} & {$[1]$} \\
[d) Zur Klärung von NI-Fragen] & {$[2]$} & {$[1]$} & {$[0]$} \\
\hline \multirow{2}{*}{ Keine Einschaltung vorgesetzter Behörden } & 51 & 33 & 21 \\
\hline \hline \multirow{2}{*}{ Verfahren gesamt } & $87,9 \%$ & $76,7 \%$ & $87,5 \%$ \\
\hline
\end{tabular}

Insgesamt wurde beim LRA-1 die Mittelinstanz mit einem Anteil von 23,3\% (10 absolut) der Verfahren häufiger angerufen, als dies beim GAA-

222 In diesem Zusammenhang ist festzuhalten, daß in keinem einzigen Fall der vorliegenden Untersuchung eine vorgesetzte Behörde von sich aus in ein laufendes Verfahren eingegriffen hat. Allgemein gehaltene, also nicht auf einen konkreten Einzelfall gemünzte Erlasse sind dabei nicht berücksichtigt.

223 Nicht dieser Kategorie zugerechnet wurden zwei das LRA-1 betreffende Fälle, in denen das Regierungspräsidium als Vertragspartner eines öffentlich-rechtlichen Vertrags notwendig in das Verfahren einzubeziehen war. 
$\mathrm{N}$ mit einem Anteil von 12,1\% (7 absolut) der Fall war. Die Differenz bleibt aber unterhalb der statistischen Signifikanzgrenze. ${ }^{224}$

Schlüsselt man dabei die Anfragen nach ihrem jeweiligen Anlaß auf (Kategorien a bis d im Kleindruck), ergibt sich folgendes Bild. Beim LRA-1 dienten sie überwiegend (8) als Mittel zur Beendigung von Kontroversen, wobei ausschließlich (immissionsschutzrechtliche) I-Kontroversen (Kategorie a), nicht aber (nicht-immissionsschutzrechtliche) NI-Kontroversen (Kategorie b) betroffen waren. Nur in der Minderzahl (2) wollte die Behörde eigener Unsicherheit über die gültige Rechtslage abhelfen, einmal in einer immissionsschutzrechtlichen Frage (Kategorie c), das andere Mal in einer andere Belange betreffenden Rechtsfrage (Kategorie d). Demgegenüber dienten die Anfragen an die Mittelinstanz beim GAA-N zu nahezu gleichen Teilen der Bewältigung von Konflikten (4) - darunter vorrangig solche nicht-immissionsschutzrechtlicher Natur (3) - und der Abklärung (3) eines immissionsschutzrechtlichen (1) bzw. anderweitigen Rechtsproblems (2).

Die nach diesen Befunden naheliegende Vermutung, daß beim LRA-1 die Einschaltung einer vorgesetzten Behörde als Instrument der Beendigung speziell von immissionsschutzrechtlichen Konflikten eine sichtlich größere Rolle spielt als beim GAA-N, läßt sich untermauern, wenn man die betreffenden Fälle auf die jeweilige Gesamtzahl der mit Konflikten einhergegangenen Verfahren bezieht. Tab. 26.1 zeigt das Ergebnis.

Tab. 26.1

Einschaltung vorgesetzter Behörden in immissionsschutzrechtlichen Konflikten

\begin{tabular}{|l|c|c|c|}
\cline { 2 - 4 } \multicolumn{1}{c|}{} & $G A A-N$ & LRA-1 & LRA-2* \\
\hline Einschaltung vorgesetzter Behörden & 1 & 8 & 2 \\
& $4,5 \%$ & $25,8 \%$ & $11,1 \%$ \\
\hline \multirow{2}{*}{ Keine Einschaltung vorgesetzter Behörden } & 21 & 23 & 16 \\
& $95,5 \%$ & $74,2 \%$ & $88,9 \%$ \\
\hline \hline Verfahren gesamt* & 22 & 31 & 18 \\
\hline
\end{tabular}

* Trotz der für eine Prozentuierung kritischen (niedrigen) Gesamtfallzahlen erscheint diese zulässig, da sie in erster Linie der Vergleichbarkeit der absoluten Häufigkeiten dient und auf einem vergleichbaren absoluten Niveau der zugehörigen Grundgesamtheiten basiert.

$224 \mathrm{chi}^{2}=2,20<2,71$ für $\mathrm{p}=0,1$ und $\mathrm{df}=1$. 
Beim LRA-1 wurde in einem Viertel $(25,8 \%)$ der immissionsschutzrechtlichen Konflikte die Lösung an die Mittelinstanz delegiert, beim GAA-N lediglich in einem Fall. Die Differenz ist statistisch signifikant. ${ }^{225} \mathrm{Im}$ Umgang mit den nicht-immissionsschutzrechtlichen Konflikten unterscheiden sich die beiden Ämter dagegen nur unwesentlich. ${ }^{226}$ Von daher dürfte die Neigung des LRA-1, sich vorzugsweise in I-Konflikten an vorgesetzte Stellen zu wenden, in erster Linie auf die modellbedingte Auslagerung der immissionsschutzrechtlichen Fachkompetenz zurückzuführen sein. Deren institutionelle Verselbständigung stellt der Genehmigungsbehörde auf dem dieser formell zugewiesenen Entscheidungssektor einen faktisch gleich kompetenten, wenn nicht (zumindest bereichsweise) überlegenen »Mitspieler« zur Seite. Die behördliche Binnendifferenzierung durch Spezialisierung geht jedoch nicht, wie man hätte erwarten können, mit einer wechselseitigen Entlastung in der Erarbeitung der Entscheidungsgrundlagen einher. Sie scheint, im Gegenteil, in dieser Hinsicht einer Erweiterung der Komplexität i.S. einer »Verkomplizierung « Vorschub zu leisten. Dies zieht nicht allein, wie gezeigt, eine höhere Konflikthäufigkeit im immissionsschutzrechtlichen Bereich nach sich. Es mindert auch die Selbststeuerungsfähigkeit des behördlichen Entscheidungssystems mit der Folge, daß Problemlösungen auf die übergeordnete Hierarchieebene verlagert werden (müssen).

\subsection{Revision von Genehmigungsentwürfen}

Dem Erlaß des Genehmigungsbescheids, der das Verfahren abschließt, hat die niedersächsische Genehmigungsbehörde genau in der Hälfte der Fälle die Übersendung eines Entwurfs des endgültigen Bescheids vorgeschaltet. ${ }^{227}$ Es handelt sich dabei, wie bereits kurz erwähnt, um eine hausintern geübte Praxis des GAA-N. ${ }^{228}$ Die Entwurfsfassung zur Vorabinformation des Antragstellers ist zwar auch im Süden bekannt. Ein routinemäßiger Einsatz dieses rechtlich zwar nicht vorgesehenen (aber auch nicht unzulässigen) Instruments wird dort, jedenfalls in den beiden hier untersuchten

$225 \mathrm{chi}^{2}=3,64$ (Yates' Korrektur) $>2,71, \mathrm{p}=0,1, \mathrm{df}=1$. Der nächsthöhere Signifikanzwert von $\mathrm{chi}^{2}=3,84$ für $\mathrm{p}=0,05$ wird nur knapp verfehlt.

226 Hier wandte sich das GAA-N in drei der insgesamt 26 Konfliktfälle an die vorgesetzte Behörde, das LRA-1 in keinem der 24 einschlägigen Fälle (chi ${ }^{2}=1,95$ (Yates' Korrektur) $<2,71$ für $\mathrm{p}=0,1$ und $\mathrm{df}=1$ ).

227 Vgl. oben Ziff. 2.4.4, Tab. 19, Kategorie d.

228 Es handelt sich dabei aber keineswegs um eine ausgefallene »Spezialität« des GAAN. Vielmehr ist die Vorabzuleitung eines Entscheidungsentwurfs auch anderwärts eine verbreitete Praxis, vgl. dazu Bohne 1981, S. 59f. 
Landratsämtern, aber ausdrücklich abgelehnt. ${ }^{229}$ Tatsächlich ist diese Maxime lediglich in zwei Fällen (beide beim LRA-1) durchbrochen worden, wobei man dem ausdrücklichen Interesse der Antragsteller, zwei größeren Unternehmen, an einem Entwurf des Genehmigungsbescheids nachgegeben hatte.

Entsprechend dieser Ausgangssituation läßt sich die Frage nach den materiellen Auswirkungen dieses Instruments auf die abschließende Genehmigungsentscheidung nur für das niedersächsische GAA-N untersuchen.

Tab. 27

Entwurfsfassung des Genehmigungsbescheids nach Streitigkeit des Verfahrensverlaufs beim GAA-N

\begin{tabular}{|l|c|c|c|}
\hline Verfahren & $\begin{array}{c}\text { Verfahren } \\
\text { ohne Streit }\end{array}$ & $\begin{array}{c}\text { Verfahren } \\
\text { mit Streit }\end{array}$ & $\begin{array}{c}\text { Reihe } \\
\text { gesamt }\end{array}$ \\
\hline Ohne Entwurf & 14 & 15 & 29 \\
Mit Entwurf & $24,1 \%$ & $25,9 \%$ & $50,0 \%$ \\
\hline \hline Spalte gesamt & 8 & 21 & 29 \\
& $13,8 \%$ & $36,2 \%$ & $50,0 \%$ \\
\hline
\end{tabular}

Wenn auch dort die Übermittlung einer Entwurfsfassung an den Antragsteller eine hausintern anerkannte Praxis ist, steht es dem einzelnen Sachbearbeiter frei, wieweit er sich ihrer bedienen will. Eine offizielle oder auch nur inoffizielle Verpflichtung dazu besteht nicht. Als Ausdruck der Wahlfreiheit der Mitarbeiter ist der Befund zu sehen, daß das Instrument nicht durchgängig, sondern (statistisch) nur in jedem zweiten Verfahren zum Zuge kam. Dabei ist die Option zugunsten der einen oder anderen Variante nicht auf prinzipielle Präferenzen der einzelnen Sachbearbeiter zurückzuführen (in diesem Fall wäre die Option als Variable des Personals zu betrachten). Vielmehr machte die Mehrheit der Mitarbeiter von beiden Möglichkeiten Gebrauch. Dies wirft die Frage auf, nach welchen Kriterien sie ihre Wahl trafen. Eine entsprechende (statistische) Prüfung ergibt, daß dem vorausgegangenen Verfahrensverlauf der größte Einfluß auf die Entscheidung zuzuschreiben ist. Wie aus Tab. 27 hervorgeht, erhielt der Antragsteller überproportional häufig einen Entwurf des Genehmigungsbescheids

229 So lt. Auskunft des LRA-1. Für das LRA-2 dürfte ausweislich der Befunde dasselbe gelten. 
überstellt, wenn sein Verfahren mit sachlichen Auseinandersetzungen einhergegangen war (in 36,2\% der Verfahren), ${ }^{230}$ und unterproportional häufig im Fall eines konfliktfreien Verlaufs (in 13,2\% der Verfahren). ${ }^{231}$ Die Vermutung, daß größere Unternehmen bevorzugt mit einer Entwurfsfassung bedient werden, hat sich dagegen nicht bestätigt.

Mit der Information über den vorgesehenen Genehmigungsinhalt, insbesondere die ins Auge gefaßten einschränkenden Bedingungen und Auflagen, wurde dem Betreiber i.d.R. ausdrücklich die Möglichkeit eingeräumt, sich dazu innerhalb einer bestimmten Frist zu äußern. ${ }^{232}$ In welchem Maße und in welchen Punkten dieser mit Abänderungswünschen der Entwurfsfassung reagierte, zeigt Tab. 28 .

Tab. 28

Abänderungswünsche des Antragstellers gegenüber der Entwurfsfassung des Genehmigungsbescheids beim GAA-N

\begin{tabular}{|l|c|}
\cline { 2 - 2 } \multicolumn{1}{c|}{} & $\begin{array}{c}\text { Verfahren } \\
\text { mit Entwurf }\end{array}$ \\
\hline a) Keine Änderungswünsche & 15 \\
\hline b) Änderungswünsche nur bzgl. NI-Auflagen & $51,7 \%$ \\
\hline c) Änderungswünsche nur bzgl. I-Auflagen & 6 \\
\hline d) Änderungswünsche bzgl. I-/NI-Auflagen & $20,7 \%$ \\
\hline \hline Verfahren mit Entwurfsfassung (gesamt) & 2 \\
\hline \hline
\end{tabular}

Danach akzeptierte der Antragsteller in gut der Hälfte $(51,7 \%)$ der Fälle die vorgesehenen Auflagen (Kategorie a), in der knappen anderen Hälfte $(48,3 \%)$ trug er Abänderungswünsche vor (Summe der übrigen Kategorien). Die Revisionsbegehren galten dabei in 12 Fällen entweder ausschließlich (6) oder zumindest auch (6) nicht-immissionsschutzrechtlichen

230 Dabei betrafen die Streitigkeiten etwa gleich häufig den Immissionsschutz (I-Kontroversen) wie sonstige fachliche Belange (NI-Kontroversen).

231 Die Differenz liegt allerdings knapp unter der Signifikanzgrenze $\left(\mathrm{chi}^{2}=2,64<2,71\right.$ für $\mathrm{p}=0,1$ und $\mathrm{df}=1$ ).

232 In der Regel blieb es bei einer Entwurfsfassung. Lediglich in zwei Fällen wurde ein zweiter Entwurf und in einem Fall sogar ein dritter erstellt. 
Auflagen (NI-Auflagen: Kategorie b bzw. d) und in 8 Fällen entweder ausschließlich (2) oder zumindest auch (6) immissionsschutzrechtlichen Auflagen (I-Auflagen: Kategorie c bzw. d). Zeichnet sich auf der Ebene der betroffenen Fälle im Verhältnis von 12:8 bereits ein - von der theoretisch zu erwartenden Gleichverteilung allerdings nicht signifikant abweichendes Übergewicht der nicht-immissionsschutzrechtlichen gegenüber den immissionsschutzrechtlichen Änderungswünschen ab, so wird das Ausmaß vollends sichtbar, wenn man die jeweilige Anzahl der monierten Auflagen mit berücksichtigt. Hier stehen den 26 Beanstandungen von NI-Auflagen lediglich 11 Beanstandungen von I-Auflagen gegenüber, ${ }^{233}$ eine von der theoretischen Gleichverteilung signifikante Abweichung zugunsten der NI-Auflagen. ${ }^{234}$ Diese Relation unterstreicht die bereits an verschiedenen Befunden ausgemachte Tendenz, wonach beim GAA-N die fallspezifischen Belange des Immissionsschutzes in geringerem Maße als die der sonstigen Fachbehörden sachliche Kontroversen auslösten.

Im Rückblick auf den Befund, daß bei streitigem Verfahrensverlauf öfter als bei unstreitigem dem Antragsteller ein Genehmigungsentwurf zur Stellungnahme übermittelt wurde (s.o. Tab. 27), ist das folgende Ergebnis bemerkenswert. Es läuft darauf hinaus, daß Abänderungen der Entwurfsfassung unabhängig davon begehrt wurden, ob zuvor kontrovers »verhandelt « worden war oder nicht. Bei den einschlägigen 8 streitfreien Verfahren beläuft sich die Relation von Akzeptanz und Beanstandungen des Entwurfs auf $5: 3$ Fälle, bei den 21 streitigen auf $10: 11$ Fälle. ${ }^{235}$ Der Antragsteller orientiert seine Reaktion auf den Entwurf offenbar in erster Linie daran, wieweit er darin seine Interessen gewahrt findet - zumal die Äußerung von Abänderungswünschen für ihn völlig risikolos ist -, und nicht am zurückliegenden Verfahrensverlauf. Schließlich darf er sich dazu sogar geradezu ermuntert fühlen, da die Genehmigungsbehörde mit dem Entwurf ihre Bereitschaft signalisiert, über die vorgesehenen Auflagen (ggf. erneut) zu verhandeln. Mit der Entwurfsfassung provoziert die Behörde einerseits in Form von Beanstandungen überhaupt erst Konflikte oder wiederbelebt frühere Kontroversen. Auf der anderen Seite verfügt sie damit über ein effektives Instrument, formelle Widersprüche abzuwehren (s. dazu weiter unten). Die Funktion der Absorption potentieller Widersprüche dürfte allerdings nicht unabhängig sein von der Reaktion der Genehmigungsbehörde auf die Beanstandungen bzw. die Erfolgschancen der Antragsteller.

233 Inhaltlich betrafen die Monita von I-Auflagen vor allem Grenzwerte und/oder Meßnachweise für Luft/Abgas (6) und Lärm (4), die Monita von NI-Auflagen vorrangig Vorgaben zum Arbeitsschutz (7) und Brandschutz (4); Bestimmungen des Naturschutzes waren nur in einem Fall betroffen.

$234 \mathrm{chi}^{2}=6,04>5,02, \mathrm{p}=0,025, \mathrm{df}=1$.

235 Die Differenz der Relationen ist, das sei der Vollständigkeit halber ausdrücklich vermerkt, weit unterhalb jeglicher statistischen Signifikanz. 
Tab. 28.1 weist (allerdings auf der Basis einer niedrigen absoluten Gesamtfallzahl) diesen Erfolg aus, den die Abänderungswünsche insgesamt verbuchen konnten.

In 11 Fällen und damit in der Mehrheit der »Einsprüche« hat sich diese Option für den Antragsteller ausgezahlt (Kategorien a plus b). Dabei erging zum (kleineren) Teil (4 Fälle) vollständige »Abhilfe« (Kategorie a), zum (größeren) Teil (7) wurde zumindest ein Teil der beanstandeten Auflagen entweder zurückgenommen oder wenigstens abgemildert (Kategorie b). Lediglich in den verbleibenden drei Fällen zeigten sich die jeweils zuständigen Behörden unnachgiebig und hielten an ihren Forderungen fest. Das quantitative Übergewicht der ganz oder teilweise erfolgreichen »Einsprüche« gegenüber den erfolglosen ist im Verhältnis zur theoretisch zu erwartenden Gleichverteilung statistisch signifikant. ${ }^{236}$

Tab. 28.1

Behördliche »Abhilfe« vom Antragsteller monierter Auflagen der Entwurfsfassung des Genehmigungsbescheids beim GAA-N*

\begin{tabular}{|c|c|}
\hline & \multirow[b]{2}{*}{$\begin{array}{c}\text { Entwürfe } \\
\text { mit Beanstandungen }\end{array}$} \\
\hline & \\
\hline a) Vollständige »Abhilfe« & $\begin{array}{c}4 \\
28,6 \%\end{array}$ \\
\hline b) Teilweise »Abhilfe« & $\begin{array}{c}7 \\
50,0 \%\end{array}$ \\
\hline c) Keine »Abhilfe « & $\begin{array}{c}3 \\
21,4 \%\end{array}$ \\
\hline Entwürfe mit Beanstandungen gesamt & $\begin{array}{c}14 \\
100,0 \%\end{array}$ \\
\hline
\end{tabular}

* Angesichts der niedrigen absoluten Grundgesamtheit an beanstandeten Entwürfen dient die Prozentuierung lediglich einer näherungsweisen (heuristischen) Vergleichbarkeit der auf die einzelnen Kategorien entfallenden absoluten Häufigkeiten.

Differenziert man die Erfolgsbilanz nach den beiden Gruppen der fachlich betroffenen Belange (I- bzw. NI-Auflagen), ergeben sich die in Tab. 28.2 dargestellten Erfolgsquoten. Bei den NI-Auflagen ist allerdings zu berücksichtigen, daß die Entscheidung darüber formell ebenfalls in die Kompetenz der Genehmigungsbehörde fällt. In der Praxis riskiert diese es aber kaum, sich über die Voten der für die nicht-immissionsschutzrechtlichen

$236 \mathrm{chi}^{2}=4,57>3,84, \mathrm{p}=0,05, \mathrm{df}=1$. 
Belange fachlich zuständigen Stellen ohne weiteres hinwegzusetzen. Vom Betreiber gewünschte Abänderungen von NI-Auflagen durch die Genehmigungsbehörde setzen somit faktisch eine entsprechende Einwilligung der betroffenen Fachbehörden voraus. Eine Einflußnahme der Genehmigungsbehörde auf deren Entscheidung ist insofern begrenzt.

Von den insgesamt acht »Einsprüchen«, die I-Auflagen galten, waren fünf ganz oder zumindest teilweise erfolgreich (Kategorien a und b) und drei gänzlich erfolglos (Kategorie c). Bei den insgesamt 12 gegen NI-Auflagen gerichteten »Einsprüchen« beläuft sich die entsprechende Relation auf neun zu drei Fälle. Die Differenz zwischen den beiden Verteilungen erreicht allerdings keine statistische Signifikanz. Die für die I-Auflagen zugleich auch fachlich verantwortliche niedersächsische Genehmigungsbehörde begegnet den vom Antragsteller verlangten Abänderungen nicht grundsätzlich entgegenkommender oder ablehnender als die für die NIAuflagen fachlich zuständigen sonstigen beteiligten Behörden. Dies ist freilich vor dem Hintergrund zu sehen, daß beide eher geneigt sind, ihre Anforderungen abzumildern als an ihnen festzuhalten.

\section{Tab. 28.2}

Behördliche »Abhilfe« vom Antragsteller monierter Auflagen der Entwurfsfassung des Genehmigungsbescheids nach Auflagenart beim GAA-N*

\begin{tabular}{|l|c|c|}
\cline { 2 - 3 } \multicolumn{1}{c|}{} & $\begin{array}{c}\text { Änderung } \\
\text { I-Auflagen }\end{array}$ & $\begin{array}{c}\text { Änderung } \\
\text { NI-Auflagen }\end{array}$ \\
\hline a) Vollständige "Abhilfe" & 3 & 3 \\
\hline b) Teilweise "Abhilfe" & $37,5 \%$ & $25,0 \%$ \\
\hline c) Keine "Abhilfe" & 2 & 6 \\
\hline \hline & $25,0 \%$ & $50,0 \%$ \\
\hline Monierte Entwürfe (gruppiert) gesamt & 3 & 3 \\
\hline
\end{tabular}

Angesichts der niedrigen absoluten Grundgesamtheit an beanstandeten Entwürfen dient die Prozentuierung lediglich einer näherungsweisen (heuristischen) Vergleichbarkeit der auf die einzelnen Kategorien entfallenden absoluten Häufigkeiten.

Das Ergebnis, daß die Antragsteller mit ihren Einwendungen gegen den Entwurf mehrheitlich die Genehmigungsbedingungen zu ihren Gunsten revidieren konnten - wenn auch nur zum kleineren Teil im vollen, gewünsch- 
ten Umfang -, dürfte auch die Eignung der Entwurfsfassung als Instrument der Widerspruchsprophylaxe (mit) erklären. Tab. 29 zeigt die Beziehung zwischen (formellem) Widerspruch gegen den (endgültigen) Genehmigungsbescheid und vorangegangener Entwurfsfassung.

Tab. 29

Widersprüche nach Entwurfsfassung des Genehmigungsbescheids beim GAA-N

\begin{tabular}{|l|c|c|c|}
\cline { 2 - 4 } \multicolumn{1}{c|}{} & $\begin{array}{c}\text { Verfahren } \\
\text { mit Entwurf }\end{array}$ & $\begin{array}{c}\text { Verfahren } \\
\text { ohne Entwurf }\end{array}$ & Reihe gesamt \\
\hline Widerspruch & 3 & 12 & 15 \\
\hline Kein Widerspruch & $5,2 \%$ & $20,7 \%$ & $25,9 \%$ \\
\hline \hline \multirow{2}{*}{ Spalte gesamt } & 26 & 17 & 43 \\
\hline \hline
\end{tabular}

Während die 29 Antragsteller, die eine Entwurfsfassung erhalten hatten, lediglich in drei Fällen (5,2\% aller bzw. 10,3\% der einschlägigen Verfahren) Widerspruch gegen den abschließenden Genehmigungsbescheid einlegten, taten dies dagegen 12 (20,7\% aller bzw. 41,4\% der einschlägigen Verfahren) der gleichfalls 29 Personen umfassenden Gruppe der anderen Antragsteller, die keine Gelegenheit zu einer Stellungnahme zum vorgesehenen Genehmigungsinhalt bekommen hatten. ${ }^{237}$ Der Zusammenhang zwischen Widerspruchsverzicht und informellem Vorabbescheid ist statistisch hochsignifikant. ${ }^{238}$

Nimmt man an, daß es sich bei den 14 Einwendern gegen vorgesehene Genehmigungsauflagen in den »Verfahren mit Entwurf « (s. oben Tab. 28) um potentielle Widerspruchskandidaten handelt, ${ }^{239}$ ergibt sich für die Gesamtgruppe dieser Verfahren eine Widerspruchsneigung, die an diejenige aufschließt, die in der gleich großen anderen Gruppe der »Verfahren ohne

237 In dieser Gruppe befindet sich außerdem der (einzige) Fall, in dem der Antragsteller ausdrücklich Rechtsmittelverzicht erklärt hatte. Seine betriebliche Planung erforderte, so seine Begründung, die Genehmigung umgehend in Anspruch nehmen zu können.

$238 \mathrm{chi}^{2}=7,28>6,64, \mathrm{p}=0,01, \mathrm{df}=1$. Siehe hierzu auch näher unten Ziff. 3.4.1.

239 Die Frage, ob diese Einwender im Falle, daß sie keinen Entwurf erhalten hätten, tatsächlich auch (formellen) Widerspruch eingelegt hätten, zielt auf einen hypothetischen Sachverhalt, für den der Beweis zwangsläufig nicht angetreten werden kann. 
Entwurf « (29) mangels Alternative im Wege des (förmlichen) Rechtsmittels verwirklicht wurde. Die über das Instrument der Entwurfsfassung erzielte $»$ Absorption « potentieller Widersprüche ist nicht unbedingt daran gebunden, daß die Behörde die Beanstandungen des Betreibers durch Rücknahme ihrer Anforderungen gegenstandslos macht. Wie oben (Tab. 28.1) gezeigt, geschah dies nur in der Minderzahl (4) der einschlägigen Fälle. In sieben der übrigen 10 Fälle akzeptierten die Antragsteller (im förmlichen Sinne) widerspruchslos, daß ihre Änderungswünsche teilweise (4) oder sogar gänzlich (3) unerfüllt blieben. Das deutet darauf hin, daß die prophylaktische Wirkung der Entwurfsfassung zu einem guten Teil auch auf der bloßen behördlichen Bereitschaft beruht, sich einer Kritik zu stellen und die vorgesehene Entscheidung nochmals zu überprüfen. ${ }^{240}$ Lediglich die noch verbleibenden restlichen drei Fälle, in denen jeweils die beanstandeten Auflagen nicht gänzlich, sondern nur partiell zurückgenommen worden waren, zogen förmliche Widerspruchsverfahren nach sich. Möglicherweise rechnet sich ein Antragsteller, der bei der Auseinandersetzung um die Entwurfsfassung der Behörde gewisse Zugeständnisse hat abringen können, weitere Erfolgschancen mit der Einlegung eines Widerspruchs aus (den er bei signalisiertem Entgegenkommen seitens der Behörde wieder zurücknehmen kann). Demgegenüber scheint das Kalkül eines Antragstellers, dem jegliches behördiiches Entgegenkommen versagt blieb, offenbar weniger optimistisch auszufallen. Tatsächlich konnten die drei Widerspruchsfüh$\operatorname{rer}^{241}$ in dem anschließenden Rechtsmittelverfahren einen weiteren Erfolg für sich verbuchen, zwei von ihnen einen (neuerlichen) teilweisen, der dritte einen einschränkungslosen. Dies ist allerdings vor dem Hintergrund zu sehen, daß die Widersprüche aus der Gruppe der »Verfahren ohne Entwurf « in vergleichbarer Weise zugunsten der Betreiber ausgingen (s. dazu näher unten Ziff. 3.4.2).

Die baden-württembergischen Genehmigungsbehörden, die die Überstellung einer Entwurfsfassung an den Antragsteller mit dem Argument, damit überhaupt erst Änderungswünsche zu provozieren, grundsätzlich ablehnen, begeben sich mit dem Verzicht auf dieses Mittel aber zugleich auch der Möglichkeit, vorhandenes Protestpotential gegen die Genehmigungsauflagen noch im Verfahren selbst aufzufangen bzw. zu absorbieren. ${ }^{242} \mathrm{Im}$ Ergebnis riskieren sie mit ihrer »Verfahrensphilosophie«, so ließe sich mit Blick auf die Befunde für das einer anderen »Philosophie« folgende GAA-

240 Bohne 1981, S. 60, spricht in diesem Zusammenhang von einer Art »vorweggenommene $(\mathrm{m})$ Widerspruchsverfahren «.

241 Bei allen dreien handelt es sich um ein mittelgroßes Unternehmen.

242 Die erwähnten zwei Ausnahmefälle, in denen das LRA-1 dem Antragsteller auf dessen ausdrücklichen Wunsch hin eine Entwurfsfassung aushändigte, nahmen folgenden Ausgang. Im einen Fall beanstandete der Betreiber lediglich eine nicht-immissionsschutzrechtliche Auflage, die die zuständige Fachbehörde daraufhin aufhob, was einen förmlichen Widerspruch gegenstandslos machte. Im anderen Fall drang 
$\mathrm{N}$ vermuten, eine höhere Zahl von formellen Widersprüchen. Ob bzw. wieweit das tatsächlich der Fall ist, wird weiter unten (Ziff. 3.4.1) zu zeigen sein.

\subsection{Genehmigungsauflagen}

Inhaltlicher Kern des abschließend erlassenen (formellen) Genehmigungsbescheids sind die auf die beantragte Anlage hin spezifizierten Anforderungen, die der Genehmigungsinhaber bei deren baulich-technischer Ausstattung und/oder im laufenden Betrieb zu erfüllen hat. Dem Untersuchungsziel entsprechend interessieren unter diesen als Nebenbestimmungen $(\S 12$ BImSchG) bezeichneten individuellen Anforderungen hauptsächlich die immissionsschutzrechtlichen Auflagen, die vom Betreiber ein bestimmtes Tun oder Unterlassen verlangen. ${ }^{243}$ Wie schon bei der Analyse des Verfahrensablaufs, die aus Vergleichsgründen die Beteiligung der sonstigen, nicht-immissionsschutzrechtliche Belange vertretenden Fachbehörden einbezogen hat, wird auch deren Auflagenerteilung hier mit berücksichtigt. Auf diese Weise soll der Möglichkeit Rechnung getragen werden, daß Unterschiede bei den immissionsschutzrechtlichen Auflagen weniger aus der Verschiedenartigkeit der Genehmigungsmodelle resultieren als vielmehr aus davon unabhängigen unterschiedlichen Randbedingungen. $\mathrm{Zu}$ denken wäre dabei etwa an eine unterschiedliche Zusammensetzung der Regelungsmaterien im Hinblick auf deren fachspezifischen »Auflagenbedarf« oder auch an im Nord-Süd-Vergleich unterschiedlich »strenge « (informelle) Maßstäbe, die jeweils fachbehördenübergreifend die Praxis der Auflagenerteilung steuern.

Zunächst wird deshalb geprüft, ob bzw. inwieweit sich die vorherrschend beteiligten sonstigen Fachbehörden beider Modelle des Instruments der Auflagen in jeweils vergleichbarer Weise bedienten. Vor diesem Hintergrund wird dann die Handhabung der immissionsschutzrechtlichen Auflagen untersucht. Die Datenanalyse stützt sich wie bisher vorrangig auf die für das GAA-N und das LRA-1 ermittelten Befunde.

der Betreiber auf Änderungen sowohl nicht- als auch immissionsschutzrechtlicher Auflagen. Während die zuständige (sonstige) Fachbehörde zumindest teilweise nachgab, bestand das GAA-1 auf seiner Forderung (dem sich das LRA-1 anschloß). Widerspruch erging gleichwohl nicht.

243 Außer den Auflagen umfaßt der Begriff der Nebenbestimmungen auch Bedingungen und Befristungen. Von letzteren unterscheiden sich Auflagen dadurch, daß sie selbständig (behördlicherseits) durchsetzbare Forderungen enthalten, deren Nichterfüllung die Wirksamkeit der Genehmigung aber nicht berührt. Vgl. im einzelnen die Erläuterung in Hansmann 1994, S. 27 f. 


\subsubsection{Nicht-immissionsschutzrechtliche (sonstige) Auflagen}

Tab. 30 zeigt die Häufigkeiten der Verfahren, in denen die hauptsächlich beteiligten sonstigen Fachbehörden einschlägige Auflagen für die Genehmigung erteilt haben. Die relativen Anteile beziehen sich dabei auf die jeweiligen Grundgesamtheiten der Verfahren. Das gleiche gilt für die (im Kleindruck ausgewiesene) Beteiligung der zugehörigen Fachbehörden. ${ }^{244}$ Aus ähnlich hohen Verfahrensanteilen in einer Auflagenkategorie läßt sich ohne weiteres nur dann auf eine vergleichbare »Auflagenneigung « der jeweils zuständigen Fachbehörden schließen, wenn diese in beiden Modellen auch ähnlich häufig in den Verfahren präsent waren.

Diese Konstellation ist offenkundig bei den bauordnungsrechtlichen Auflagen gegeben. Sie wurden mit einer Differenz von 1,8 Prozentpunkten nahezu gleichermaßen beim GAA-N (48,3\% der Verfahren) und beim LRA$1(46,5 \%)$ erteilt bei ebenfalls nahezu gleicher Bauamtspräsenz von 89,7\% bzw. 90,7\% (entsprechend 1,0 Prozentpunkte Differenz). Die Quoten für die wasser- und bodenschutzbezogenen Auflagen differieren mit 6,2 Prozentpunkten zwischen dem GAA-N $(69,0 \%)$ und dem LRA-1 $(62,8 \%)$ etwas stärker, wobei die Beteiligungsquoten der zugehörigen Fachbehörden von $89,7 \%$ bzw. $90,7 \%$ ebenfalls nur um 1,0 Prozentpunkte - wenngleich in gegenläufiger Richtung - voneinander abweichen. ${ }^{245}$

Bei den übrigen nicht-immissionsschutzrechtlichen Materien stehen den Auflagenanteilen größere Divergenzen in der Verfahrensbeteiligung der jeweiligen Fachbehörden gegenüber. Von daher läßt sich die »Auflagenneigung « nicht mehr hilfsweise aus den auf den Grundgesamtheiten basierenden Anteilswerten ablesen. Vergleichbar sind die Häufigkeiten der Auflagenerteilung erst dann, wenn man sie in Beziehung zur (im jeweiligen Bereich jeweils unterschiedlichen) Beteiligung der zugehörigen Behörde setzt. ${ }^{246}$ Die statistische Prüfung der auf dieser Basis errechneten Auflagenanteile ergibt bei zwei weiteren Belangen, beim Brandschutz und beim Naturschutz, keine signifikanten Differenzen. ${ }^{247}$ Wenn auch die Auflagenhäu-

244 Vgl. zu den relativen (und absoluten) Anteilen der Fachbehördenbeteiligung oben Ziff. 2.3.2, Tab. 14. Der Fall, daß keinerlei fachbehördliche Auflagen erteilt wurden, bildet bei allen drei Genehmigungsbehörden die seltene Ausnahme.

245 Die Differenz bei den Auflagenanteilen ist - unter Berücksichtigung der zugehörigen fachbehördlichen Beteiligungsanteile - statistisch nicht signifikant $\left(\right.$ chi $^{2}=0,68$ $<2,71$ für $\mathrm{p}=0,1$ und $\mathrm{df}=1$ ).

246 Dies gilt selbstverständlich auch für die bereits angesprochenen bauordnungs- und wasser-/bodenschutzrechtlichen Belange, konnte dort aber wegen der jeweils nur geringen Differenz der Behördenbeteiligung in der Darstellung vernachlässigt werden (nicht aber bei der statistischen Prüfung der Auflagendifferenz bei den wasser/bodenschutzrechtlichen Belangen, s. vorhergehende Anm.).

247 Brandschutz: chi $^{2}=0,98$, Naturschutz: chi $^{2}=1,47$ (Yates' Korrektur), jeweils $<2,71$ für $\mathrm{p}=0,1$ und $\mathrm{df}=1$. 
Tab. 30

Nicht-immissionsschutzrechtliche Auflagen (Auswahl - alphabetisch sortiert)

\begin{tabular}{|c|c|c|c|}
\hline & \\
\hline & $G A A-N$ & $L R A-I$ & LRA-2 \\
\hline $\begin{array}{l}\text { Abfall } \\
\text { [Fachbehördenbeteiligung] }\end{array}$ & $\begin{array}{c}28 \\
48,3 \% \\
{[72,4 \%]}\end{array}$ & $\begin{array}{c}1 \\
2,3 \% \\
{[7,0 \%]}\end{array}$ & $\begin{array}{c}7 \\
29,2 \% \\
{[33,3 \%]}\end{array}$ \\
\hline $\begin{array}{l}\text { Arbeits-/gewerbl. Gesundheitsschutz } \\
\text { [Fachbehördenbeteiligung] }\end{array}$ & $\begin{array}{c}50 \\
86,2 \% \\
{[100,0 \%]}\end{array}$ & $\begin{array}{c}31 \\
72,1 \% \\
{[100,0 \%]}\end{array}$ & $\begin{array}{c}19 \\
79,2 \% \\
{[100,0 \%]}\end{array}$ \\
\hline $\begin{array}{l}\text { Bauordnung } \\
\text { [Fachbehördenbeteiligung] }\end{array}$ & $\begin{array}{c}28 \\
48,3 \% \\
{[89,7 \%]}\end{array}$ & $\begin{array}{c}20 \\
46,5 \% \\
{[90,7 \%]}\end{array}$ & $\begin{array}{c}8 \\
33,3 \% \\
{[95,8 \%]}\end{array}$ \\
\hline $\begin{array}{l}\text { Brandschutz } \\
\text { [Fachbehördenbeteiligung] }\end{array}$ & $\begin{array}{c}31 \\
53,4 \% \\
{[81,0 \%]}\end{array}$ & $\begin{array}{c}17 \\
39,5 \% \\
{[72,1 \%]}\end{array}$ & $\begin{array}{c}13 \\
54,2 \% \\
{[58,3 \%]}\end{array}$ \\
\hline $\begin{array}{l}\text { Naturschutz } \\
\text { [Fachbehördenbeteiligung] }\end{array}$ & $\begin{array}{c}9 \\
15,5 \% \\
{[29,3 \%]}\end{array}$ & $\begin{array}{c}8 \\
18,6 \% \\
{[23,3 \%]}\end{array}$ & $\begin{array}{c}0 \\
0,0 \% \\
{[25,0 \%]}\end{array}$ \\
\hline $\begin{array}{l}\text { Wasser-/Bodenschutz } \\
\text { [Fachbehördenbeteiligung] }\end{array}$ & $\begin{array}{c}40 \\
69,0 \% \\
{[89,7 \%]}\end{array}$ & $\begin{array}{c}27 \\
62,8 \% \\
{[90,7 \%]}\end{array}$ & $\begin{array}{c}17 \\
70,8 \% \\
{[100,0 \%]}\end{array}$ \\
\hline $\begin{array}{l}\text { Kommunale Anforderungen } \\
\text { [Gemeindenbeteiligung] }\end{array}$ & $\begin{array}{c}4 \\
6,9 \% \\
{[32,8 \%]}\end{array}$ & $\begin{array}{c}5 \\
11,6 \% \\
{[97,7 \%]}\end{array}$ & $\begin{array}{c}1 \\
4,2 \% \\
{[95,8 \%]}\end{array}$ \\
\hline Keine Auflagen & $\begin{array}{c}1 \\
1,7 \%\end{array}$ & $\begin{array}{c}3 \\
7,0 \%\end{array}$ & $\begin{array}{c}0 \\
0,0 \%\end{array}$ \\
\hline Verfahren gesamt* & $\begin{array}{c}58 \\
100,0 \%\end{array}$ & $\begin{array}{c}43 \\
100,0 \%\end{array}$ & $\begin{array}{c}24 \\
100,0 \%\end{array}$ \\
\hline
\end{tabular}

* Da sich die Kategorien nicht wechselseitig ausschließen (Mehrfachnennungen), liegt die Summe der absoluten bzw. relativen Häufigkeiten über der Gesamtzahl der Verfahren bzw. über $100 \%$. 
figkeiten in den genannten vier Fachbelangen nicht statistisch signifikant voneinander abweichen, so folgen sie insofern einer einheitlichen Tendenz, als sich die jeweils zugehörige Fachbehörde beim GAA-N stets etwas »auflagengeneigter « zeigt als das entsprechende Gegenstück beim LRA-1. Davon auszunehmen ist lediglich der - vergleichsweise deutlich weniger häufig beteiligte - Naturschutz. Er wurde beim LRA-1 gegenüber dem GAA$\mathrm{N}$ einerseits in geringerem Maße konsultiert, andererseits wartete er in diesen Fällen öfter mit Auflagen auf. ${ }^{248}$

Nicht in das bisherige Bild einer (statistisch) gleichartigen Auflagenpraxis der beteiligten baden-württembergischen und niedersächsischen sonstigen Fachbehörden fügen sich die beiden noch offenen Belange des Abfallrechts und des Arbeitsschutzes (einschl. des gewerblichen Gesundheitsschutzes).

Beim Abfallrecht basiert die Auflagenerteilung beim LRA-1 (wie auch beim LRA-2) auf einer nicht nur relativ zum GAA-N, sondern auch absolut äußerst geringen Behördenbeteiligung, auf deren mögliche Gründe schon näher eingegangen wurde (vgl. oben Ziff. 2.3.2). Von daher fehlt es bereits an den quantitativen Voraussetzungen für eine vergleichende Betrachtung der geforderten einschlägigen Auflagen.

Anders stellt sich die Situation beim Arbeitsschutz dar. Für ihn ist bei beiden Modellen das GAA zusätzlich zü den immissionsschützrechtlichen Belangen fachlich zuständig. Auf Grund dieser im Norden wie im Süden gleichermaßen gegebenen fachlichen Doppelkompetenz hat das in immissionsschutzrechtlichen Verfahren jeweils ausnahmslos beteiligte GAA immer auch die arbeitsschutzrechtlichen Belange zu prüfen. Bei von daher gegebener identischer Fachbehördenbeteiligung erteilte das dem LRA-1 zugehörige GAA-1 mit einem Anteil von 72,1\% der Verfahren deutlich weniger Auflagen als das GAA-N mit einer entsprechenden Quote von 86,2\%. Die Differenz von 14,1 Prozentpunkten ist statistisch signifikant. ${ }^{249} \mathrm{Im}$ Hinblick auf den nachfolgenden Vergleich der immissionsschutzrechtlichen Auflagen ist es wichtig festzuhalten, daß beide Ämter intern branchenspezifisch organisiert sind und keine Separierung nach Arbeits- und Immissionsschutz praktizieren (»Ein Mann-ein Betrieb«-Prinzip). Das bedeutet im konkreten Fall, daß beide Belange von jeweils ein und demselben Sachbearbeiter geprüft werden.

Die von den Genehmigungsbehörden unterschiedlich gehandhabte Einbeziehung der - nicht den Fachbehörden im strikten Sinn zuzurechnenden - Gemeinden wurde bereits angesprochen (vgl. oben Ziff. 2.3.2). Ihrer rou-

248 Beim LRA-1 in 8 von insgesamt 10, beim GAA-N in 9 von insgesamt 17 einschlägigen Fällen. Wegen der geringen absoluten Beteiligungsrate in beiden Modellen bleibt die Differenz bei der Auflagenerteilung deutlich unterhalb statistischer Signifikanz (vgl. vorhergehende Anm.).

$249 \mathrm{chi}^{2}=3,11>2,71, \mathrm{p}=0,1, \mathrm{df}=1$. 
tinemäßigen Anhörung im Süden steht eine selektive, fallspezifisch gewährte Partizipation im Norden gegenüber. Angesichts dieser divergierenden Ausgangssituation dürfte es wenig überraschen, daß in der Gesamtheit der Verfahren die Kommunen beim LRA-1 vergleichsweise häufiger zusätzliche Anforderungen an die Genehmigungserteilung erhoben als beim GAA-N (11,6\% zu 6,9\%). Man hätte jedoch mit einer noch größeren (und auch signifikanten) Differenz zugunsten des LRA-1 rechnen dürfen. Indessen liegt der Anteil an kommunalen Auflagen, wenn man ihn auf die jeweilige gemeindliche Beteiligungsquote bezieht, beim GAA-N über dem beim LRA-1 (21,1\% zu 11,9\%) - eine nicht unbedingt zu erwartende Differenz, womit weniger ihr (statistisch nicht signifikantes) Ausma $\beta^{250}$ als vielmehr ihre Bilanz »zugunsten « des GAA-N gemeint ist.

Die Analyse der im Genehmigungsbescheid niedergelegten nicht-immissionsschutzrechtlichen fallspezifischen Anforderungen läßt sich abschlieBend wie folgt resümieren. Insgesamt unterscheiden sich die an den Verfahren vorherrschend beteiligten Fachbehörden in Baden-Württemberg in ihrer »Auflagenneigung « nicht in statistisch relevanter Ausprägung von ihren niedersächsischen Pendants. Davon auszunehmen ist das GAA, das sich in seiner fachlichen Zuständigkeit für die arbeitsschutzrechtlichen Belange im Norden als sichtlich »strenger« erweist als im Süden.

\subsubsection{Immissionsschutzrechtliche Auflagen}

Die Belange, die am häufigsten Gegenstand immissionsschutzrechtlicher Auflagen waren, und die Anteile der jeweils davon betroffenen Verfahren weist Tab. 31 aus.

Ein erster vergleichender Blick auf die Häufigkeitsverteilung ergibt, daß in jeder der aufgeführten Kategorien beim LRA-1 jeweils (relativ) mehr Verfahren mit entsprechenden Auflagen belastet wurden als beim GAA-N. Im einzelnen fallen dabei die anteiligen Differenzen bei den verschiedenen Auflagenarten unterschiedlich hoch aus. Die geringste Abweichung von 1,2 Prozentpunkten zeigen Grenzwertfestsetzungen für Lärmimmissionen mit einer Quote von $51,2 \%$ beim LRA-1 gegenüber $50,0 \%$ beim GAA-N. Es folgen - in aufsteigender Linie - mit einer Differenz von 4,1 Prozentpunkten Grenzwertfestsetzungen für Schadstoffkonzentrationen in der Abluft (55,8\% gegenüber 51,7\%), mit einer Differenz von 6,1 Prozentpunkten Sicherheitstechnik (23,3\% gegenüber 17,2\%) und mit einer Differenz von 7,4 Prozentpunkten baulich-technische Maßnahmen zur Reduktion von Emis-

$250 \mathrm{chi}^{2}=0,38$ (Yates' Korrektur) $<2,71$ für $\mathrm{p}=0,1$ und $\mathrm{df}=1$. 
sionen über die Abluft (41,9\% gegenüber 34,5\%). Sämtliche Unterschiede bleiben allerdings unterhalb der Signifikanzgrenze. ${ }^{251}$

Tab. 31

Immissionsschutzrechtliche Auflagen (ohne Meß- und Prüfnachweise) $)^{252}$

\begin{tabular}{|l|c|c|c|}
\cline { 2 - 4 } \multicolumn{1}{c|}{} & GAA-N & LRA-1 & LRA-2 \\
\hline Lärm: Grenzwerte & 29 & 22 & 16 \\
& $50,0 \%$ & $51,2 \%$ & $66,7 \%$ \\
\hline Lärm: Schallschutzmaßnahmen & 4 & 10 & 3 \\
& $6,9 \%$ & $22,3 \%$ & $12,5 \%$ \\
\hline Luft (Abluft): & 30 & 24 & 16 \\
Grenzwerte Schadstoffkonzentration & $51,7 \%$ & $55,8 \%$ & $66,7 \%$ \\
\hline Luft (Abluft): & 20 & 18 & 4 \\
Baulich-technische Maßnahmen & $34,5 \%$ & $41,9 \%$ & $16,7 \%$ \\
\hline Sicherheitstechnik* & 10 & 10 & 4 \\
\hline Sonstige Auflagen & $17,2 \%$ & $23,3 \%$ & $16,7 \%$ \\
\hline \hline Keine Auflagen & 7 & 22 & 3 \\
\hline \hline Verfahren gesamt** & $12,1 \%$ & $51,2 \%$ & $12,5 \%$ \\
\hline \hline
\end{tabular}

* Die sicherheitstechnischen Auflagen sind nicht sämtlich im strikten Sinne immissionsschutzrechtlicher Natur, sondern schließen (zum kleineren Teil) auch gewerberechtliche Sicherheitstechnik ein.

** Da sich die Kategorien nicht wechselseitig ausschließen (Mehrfachnennungen), liegt die Summe der absoluten bzw. relativen Häufigkeiten über der Gesamtzahl der Verfahren bzw. über $100 \%$.

Anders verhält es sich bei den geforderten (baulich-technischen) Schallschutzmaßnahmen und den sonstigen, von den anderen Kategorien nicht erfaßten Auflagen. ${ }^{253}$ Beide erreichen beim LRA-1 signifikant höhere Anteile als beim GAA-N. Schallschutzmaßnahmen wurden beim LRA-1 in

251 Der höchste Testwert von $\mathrm{chi}^{2}=1,15<2,71$ für $\mathrm{p}=0,1$ und $\mathrm{df}=1$ ergibt sich für die Differenz bei sicherheitstechnischen Auflagen.

$252 \mathrm{Zu}$ den insoweit auferlegten Nachweispflichten s. nachf. Tab. 32.

253 Der »Restkategorie« der sonstigen Auflagen zugeordnet ist ein breiteres Spektrum von insgesamt seltener erhobenen Anforderungen an den Anlagenbetrieb wie bei- 
23,3\% der Verfahren auferlegt gegenüber einem Anteil von lediglich 6,9\% beim GAA-N. ${ }^{254}$ Noch stärker divergieren die erzielten Quoten von 51,2\% bzw. $12,1 \%$ bei den sonstigen Auflagen. ${ }^{255}$

Obwohl das LRA-1 bzw. »sein « zuständiges GAA-1 in allen Kategorien immissionsschutzrechtlicher Auflagen höhere Werte erzielt und sich insofern »auflagengeneigter « zeigt als das GAA-N, weichen die Anteile der Verfahren, die bei beiden Ämtern von jeglichen Auflagen verschont blieben, nur geringfügig voneinander ab $(11,6 \%$ gegenüber $15,5 \%)$. Dies läßt darauf schließen, daß die größere Bereitschaft des GAA-1 zur Erteilung von Auflagen weniger einem höheren Aufkommen an Anlagen mit immissionsschutzrechtlichem Reglementierungsbedarf zuzurechnen ist als vielmehr einer häufigeren Kumulation von gleichgerichteten Auflagen i.S. einer Absicherung der im jeweiligen Bereich angestrebten Umweltverträglichkeit des Anlagenbetriebs. Das zeigt sich nicht zuletzt etwa auch darin, daß Grenzwertfestsetzungen für Lärm und Schadstoffkonzentrationen in der Abluft beim GAA-1 häufiger als beim GAA-N von der Verpflichtung auf baulich-technische Emissionsminderungsmaßnahmen flankiert wurden, um so einem Überschreiten der Grenzwerte vorzubeugen.

Die im Hinblick auf umweltschädliches Betreiberverhalten prohibitiver orientierte Auflagenpraxis im Süden kommt auch bei den Forderungen nach Meß- und Prüfnachweisen zum Ausdruck, die in Tab. 32 wiedergegeben sind.

Wie zu sehen ist, verlangte das GAA-1 häufiger als das GAA-N Meßnachweise für Schallimmissionen (Lärm) und Schadstoffemissionen (Luft bzw. Abluft), ${ }^{256}$ sicherheitstechnische Prüfungen ${ }^{257}$ sowie sonstige (anlagenbezogene Funktions-) Nachweise. ${ }^{258}$ Auch hier sind die Abweichungen

spielsweise Beschränkungen der Anlagenkapazität und der Betriebszeiten, Fristsetzungen für baulich-technische Emissionsschutzmaßnahmen, vom Betreiber durchzuführende regelmäßige Wartungs- und Kontrollmaßnahmen oder auch die Vermeidung von Belästigungen der Nachbarn durch betriebliche Lichtquellen.

$254 \mathrm{chi}^{2}=5,54>5,02, \mathrm{p}=0,025, \mathrm{df}=1$.

$255 \mathrm{chi}^{2}=21,19>10,8, \mathrm{p}=0,001, \mathrm{df}=1$.

256 In beiden Rubriken handelt es sich ausschließlich um förmliche Meßnachweise nach $\S 26 \mathrm{BImSchG}$, d.h. die Messungen (ggf. auch Berechnungen) müssen von einer öffentlich anerkannten, von der zuständigen obersten Landesbehörde bekanntgegebenen Stelle durchgeführt werden. Die Kosten trägt der Betreiber.

257 Auch hier handelt es sich um förmliche Prüfungen gem. § 29a BImSchG, d.h. sie sind von einer öffentlich anerkannten, von der zuständigen obersten Landesbehörde bekanntgegebenen Stelle oder durch andere gesetzlich anerkannte Sachverständige vornehmen zu lassen, ebenfalls auf Kosten des Betreibers.

258 Darunter zählen im einzelnen einmalige Nachweise wie Belege zur Wirksamkeit der gewählten Abluftreinigungsvorrichtung oder der Abscheideleistung der vom Betreiber vorgesehenen Filteranlage (etwa durch Herstellerbescheinigung), Nachweise der chemischen Zusammensetzung eines eingesetzten Stoffs oder regelmäßige Nachweise im Hinblick auf die Einhaltung der maximal erlaubten Anlagenleistung 
in den einzelnen Nachweiskategorien unterschiedlich stark ausgeprägt. ÄuBerst gering fällt die Differenz (0,2 Prozentpunkte) bei den Schallimmissionsmessungen aus, die in 20,9\% der Verfahren beim LRA-1 gefordert wurden gegenüber $20,7 \%$ beim GAA-N. Kaum größer ( 0,7 Prozentpunkte) ist sie bei den sonstigen Nachweisen in Höhe von 9,3\% gegenüber 8,6\%. Eine deutlichere Abweichung (7,7 Prozentpunkte) findet sich bei den sicherheitstechnischen Prüfungen von $16,3 \%$ gegenüber $8,6 \%$, die aber gleichfalls statistisch unerheblich ist. ${ }^{259}$ Signifikant ist dagegen der Unterschied in Höhe von 16,7 Prozentpunkten bei den Emissionsnachweisen für Schadstoffkonzentrationen in der Abluft, die das LRA-1 bzw. GAA-1 in $51,2 \%$, das GAA-N lediglich in $34,5 \%$ der Verfahren dem Betreiber auferlegte. 260

Tab. 32

Immissionsschutzrechtliche Nachweispflichten: Meß- und Prüfnachweise

\begin{tabular}{|l|c|c|c|}
\cline { 2 - 4 } \multicolumn{1}{c|}{} & GAA-N & LRA-1 & LRA-2 \\
\hline \multirow{2}{*}{ Lärm } & 12 & 9 & 2 \\
& $20,7 \%$ & $20,9 \%$ & $8,3 \%$ \\
\hline \multirow{2}{*}{ Luft (Abluft) } & 20 & 22 & 10 \\
& $34,5 \%$ & $51,2 \%$ & $41,7 \%$ \\
\hline \multirow{2}{*}{ Sicherheitstechnik } & 5 & 7 & 3 \\
\hline \multirow{2}{*}{ Sonstige Nachweise } & $8,6 \%$ & $16,3 \%$ & $12,5 \%$ \\
\hline \hline \multirow{2}{*}{ Keine Nachweise } & 5 & 4 & 1 \\
& $8,6 \%$ & $9,3 \%$ & $4,2 \%$ \\
\hline \hline Endabnahme & 27 & 15 & 11 \\
[Anzeige der Betriebsaufnahme] & $46,6 \%$ & $34,9 \%$ & $45,8 \%$ \\
\hline \hline \multirow{2}{*}{ Verfahren gesamt* } & 33 & 0 & 0 \\
& $56,9 \%$ & $0,0 \%$ & $0,0 \%$ \\
\hline
\end{tabular}

* Da sich die Kategorien nicht wechselseitig ausschließen (Mehrfachnennungen), liegt die Summe der absoluten bzw. relativen Häufigkeiten über der Gesamtzahl der Verfahren bzw. über $100 \%$.

oder auch im Hinblick auf die Funktionstüchtigkeit der verlangten kontinuierlichen Meßeinrichtungen.

259 Die Testwerte lauten für Schallimmissionsnachweise $\mathrm{chi}^{2}=0,90$ und für sicherheitstechnische Prüfungen chi $^{2}=1,38$, jeweils $<2,71$ für $\mathrm{p}=0,1$ und $\mathrm{df}=1$.

$260 \mathrm{chi}^{2}=2,83>2,71, \mathrm{p}=0,1, \mathrm{df}=1$. 
Insgesamt liegt der Anteil der Verfahren mit Nachweispflichten für Lärmund/oder Luftwerte beim LRA-1 mit 60,4\% zwar deutlich über dem beim GAA-N mit 44,8\%, die Differenz (15,6 Prozentpunkte) bleibt jedoch noch (knapp) unterhalb statistischer Signifikanz. ${ }^{261}$ Eine in der Höhe vergleichbare, ebenfalls nicht ganz an die Signifikanzgrenze heranreichende anteilige Differenz von 15,2 Prozentpunkten (72,1\% gegenüber 56,9\%) ergibt sich, ${ }^{262}$ wenn man auch die Verfahren berücksichtigt, in denen der Betreiber bereits seinem Antrag gutachtliche Meßbelege auf entsprechendes behördliches Verlangen hin beigefügt hat. ${ }^{263}$ Während aber beim GAA-1 in den Verfahren mit Auflagen zur Lärm- und/oder Luftmessung mehrheitlich zusätzliche Begutachtungen der Lärm- bzw. Luftverhältnisse des Anlagenbetriebs als Antragsunterlage vorlagen, verhält es sich beim GAA-N genau umgekehrt, d.h. mehrheitlich fehlte es an derartigen Gutachten. ${ }^{264}$ Dieser Unterschied ist, wenn auch knapp, statistisch signifikant. ${ }^{265}$

Wie schon bei den in Tab. 31 dargestellten immissionsschutzrechtlichen Auflagen wurden beim LRA-1 auch weniger Verfahren von jeglichen Nachweispflichten freigestellt als beim GAA-N (34,9\% gegenüber 46,6\%). Aber auch diese - mit 11,7 Prozentpunkten im Vergleich zu dem entsprechenden Wert bei den Auflagen deutlich größere - Differenz reicht nicht an eine statistische Signifikanz heran. ${ }^{266}$ Insofern unterstreicht dieses Resultat die für die Auflagenpraxis formulierte These in analoger Weise, daß nämlich das in den verschiedenen Kategorien von Nachweispflichten ermittelte Gefälle zwischen dem baden-württembergischen und dem niedersächsischen Gewerbeaufsichtsamt weniger auf unterschiedliche, anlagenspezifisch begründete Kontrollerfordernisse zurückzuführen ist, sondern sich mehr einer den Betreiber stärker in die Pflicht der Auflagenerfüllung nehmenden und insoweit absichernden Strategie verdankt.

Dieses Ergebnis scheint auf den ersten Blick nicht in Einklang zu stehen mit einem weiteren Befund, der die sog. Endabnahme betrifft (vorletzte Zeile von Tab. 32). Es handelt sich dabei um eine in ihrem Umfang noch unbestimmt belassene Überprüfung der genehmigten Anlagenerrichtung

261 chi $^{2}=2,44<2,71$ für $p=0,1$ und $\mathrm{df}=1$. Die absoluten Häufigkeiten belaufen sich auf jeweils 26 Fälle.

$262 \mathrm{chi}^{2}=2,45<2,71$ für $\mathrm{p}=0,1$ und $\mathrm{df}=1$. Die absolute Fallzahl beträgt 31 beim LRA1 und 33 beim GAA-N.

263 Zu den im Rahmen der Vorantragsberatung bzw. der Antragsbearbeitung verlangten Gutachten vgl. oben Ziff. 1.3.4 und 2.4.4.

264 Bei gleicher absoluter Anzahl an Verfahren mit Nachweispflichten für Luft und/oder Lärmwerte von jeweils 26 erfolgte die Auflage in 16 Fällen beim LRA-1, aber nur in 10 Fällen beim GAA-N zusätzlich zu einem bereits als Antragsunterlage gelieferten einschlägigen (prognostischen) Gutachten. Entsprechend beträgt die Relation der Fälle, in denen kein Gutachten existierte, $10 \mathrm{zu} 16$.

$265 \mathrm{chi}^{2}=2,77>2,71, \mathrm{p}=0,1, \mathrm{df}=1$.

$266 \mathrm{chi}^{2}=1,38<2,71, \mathrm{p}=0,1, \mathrm{df}=1$. 
bzw. -änderung nach deren Fertigstellung und Inbetriebnahme. Als Nebenbestimmung zur Genehmigung findet sie sich nur in den niedersächsischen Bescheiden, und zwar in gut der Hälfte $(56,9 \%)$ der Verfahren. Bei beiden baden-württembergischen Genehmigungsbehörden ist dieses Instrument zwar als Möglichkeit bekannt, wird aber nicht in Anspruch genommen.

In der Ankündigung einer Endabnahme könnte man eine Art Ausgleich oder Surrogat für die festgestellte zurückhaltendere Verwendung von Nachweispflichten beim GAA-N sehen. Tatsächlich konzentrieren sich die Endabnahmen überproportional häufig auf solche Verfahren, in denen für die festgelegten Emissions- bzw. Immissionsgrenzwerte für Luft- und/oder Lärm keine Meßbelege für ihre Einhaltung gefordert wurden. ${ }^{267}$ Mehrere Gründe sprechen allerdings dagegen, die beiden Kontrollinstrumente als materielle Äquivalente anzusehen. Während Meßauflagen typischerweise in Art, Umfang und Zeitpunkt spezifiziert sind und von daher dem Betreiber wenig eigenen Handlungsspielraum bei ihrer Erfüllung belassen, ist bei der behördlichen Ankündigung einer abschließenden Gesamtüberprüfung der Anlage nach Inbetriebnahme eher das Gegenteil der Fall. Zum einen unterliegt schon deren Terminierung einer gewissen Disposition des Betreibers insofern, als es in seiner Hand liegt, welche Zeitspanne er zwischen Inbetriebnahme der Anlage und entsprechender Unterrichtung der Behörde verstreichen läßt und wieweit er darüber hinaus auch noch nach Anzeige der Betriebsaufnahme den behördlichen Abnahmetermin unter Berufung auf betriebsbedingte Umstände weiter hinausschieben kann. ${ }^{268}$ Zum zweiten ist eine solche Gesamtprüfung zwangsläufig auf Sichtkontrollen äußerlicher Betriebszustände und -abläufe (und ggf. zugehöriger technischer Unterlagen) beschränkt. Von daher dürfte sie allenfalls in Ausnahmefällen hinreichende Anhaltspunkte für überhöhte Emissionen oder Immissionen liefern, in der Regel die Frage der Grenzwerteinhaltung aber ungeprüft lassen (müssen). Werden dem Betreiber anläßlich festgestellter Mängel oder hinreichender Verdachtsmomente auf immissionsschutzwidrige Gegebenheiten Nachbesserungen und/oder Meßnachweise auferlegt, dürfte er bestrebt sein, der Behörde wenn nicht eine substantielle Minderung ihrer Forderun-

267 Der Zusammenhang zwischen fehlender Nachweispflicht der Grenzwerteinhaltung und vorgesehener Endabnahme ist statistisch signifikant $\left(\mathrm{chi}^{2}=3,45>2,71, \mathrm{p}=0,1\right.$, df $=1$ ).

268 Dabei dürfte es dem Betreiber entgegenkommen, daß das GAA-N in der Mehrzahl der Fälle die Endabnahme unter Beteiligung der übrigen am Genehmigungsverfahren beteiligten Fachbehörden vorsieht und damit das Risiko zeitverzögernder Koordinationsprobleme in Kauf nimmt. 
gen, so doch zumindest eine großzügige Frist für deren Erfüllung abzuhandeln. ${ }^{269}$

Zwar ist beim Vergleich des Instruments der Endabnahme und dem der Meßnachweise einzuräumen, daß die regelmäßige Bindung von Nachweispflichten an Fristen ${ }^{270}$ nicht bereits auch deren Einhaltung sichert und von daher der Betreiber sich auch hier in Form von (geduldeten) Fristüberschreitungen temporäre Entlastung verschaffen kann. Gleichwohl dürften dessen Dispositionsmöglichkeiten bei der zeitlich und vor allem auch inhaltlich weitgehend offengehaltenen Endabnahme im ganzen gesehen deutlich größer sein als bei den in Art, Umfang und Zeitpunkt präzisierten Meßauflagen. ${ }^{271} \mathrm{Im}$ Unterschied zu jenen zielgerichtet-problemspezifischen Kontrollen hebt sich die Endabnahme nicht grundsätzlich von einer Betriebsrevision im Rahmen der allgemeinen immissionsschutzrechtlichen Überwachungstätigkeit der Gewerbeaufsicht nach § 52 BImSchG ab. ${ }^{272}$

Die dargelegten Befunde lassen sich thesenhaft zu folgendem Fazit für die beiden Genehmigungsmodelle bündeln. Das baden-württembergische Modell begünstigt eher eine prophylaktisch-absichernde, den Antragsteller von vornherein auf »handfeste « Schutz- und Prüfmaßnahmen verpflichtende Strategie des dem LRA »zuarbeitenden « GAA. Dieses »überwälzt« so die Kontrolle der Umsetzung der Genehmigungsentscheidung in der zweiten Vollzugsstufe »vor Ort « unmittelbar auf den Betreiber und mittelbar auf die Genehmigungsbehörde, die die Einhaltung der Auflagen gegenüber dem Betreiber durchzusetzen hat. Das LRA gewinnt damit aber zu-

269 Die Verpflichtung des Betreibers zur bloßen Anzeige der Betriebsaufnahme (im Kleindruck der Kategorie »Endabnahme« zugeordnet) statuiert dagegen lediglich eine reine Informationspflicht, an die keine darüber hinausgehenden Konsequenzen geknüpft sind.

270 Die Nachweise für Lärmmessungen waren in der großen Mehrheit innerhalb von maximal sechs Monaten nach Betriebsaufnahme vorzulegen. Bei den Nachweisen für Luftmessungen war zum Teil eine längere Frist von maximal 12 Monaten vorgesehen.

271 In nicht wenigen Fällen enthalten sie detaillierte Vorgaben - wie etwa Zahl und Lage der Meßpunkte - für die Durchführung der Messung.

272 Das gilt auch für die Kosten, die in beiden Fällen grundsätzlich der Betreiber zu tragen hat ( $\$ 52$ Abs. 4 BImSchG mit gewissen, hier aber nicht relevanten Differenzierungen). - Es ist an dieser Stelle nochmals ausdrücklich zu betonen, daß weder die (fristgerechte) Erfüllung der Nachweispflichten durch den Betreiber noch die Terminierung und Durchfuihrung der Endabnahmen durch die Genehmigungsbehörde systematisch erfaßt wurden. Beide fallen in das hier als zweite Stufe des Gesetzesvollzugs bezeichnete Stadium der Durchsetzung der im Genehmigungsbescheid niedergelegten Entscheidung der ersten Vollzugsstufe, auf die sich die vorliegende Untersuchung konzentriert und auch beschränkt (vgl. oben Kap. I). Die Auswirkungen der Instrumentenwahl - Meßnachweise vs. Endabnahme - auf das Ergebnis der zweiten Stufe i.S. der damit tatsächlich erreichten Umweltverträglichkeit des Anlagenbetriebs zu analysieren, bleibt vorerst ein Forschungsdesiderat. 
gleich einen Teil seines Handlungsspielraums zurück, den es auf der ersten Vollzugsstufe mangels zureichender eigener fachlich-technischer Kompetenz faktisch an das GAA hat abtreten müssen. Demgegenüber unterstützt das niedersächsische Modell eher einen »sparsameren« Gebrauch von Vorabkontrollen zugunsten der Option einer zukünftigen, nicht näher begrenzten und ggf. umfassenden nachträglichen Überprüfung. Damit verschafft sich hier die Gewerbeaufsicht größeren Handlungsspielraum für die zweite Vollzugsstufe, wobei sie sich offenhält, wieweit sie ihn zugunsten des Betreibers ausschöpfen will.

\subsection{Förmliche Widersprüche und ihr Ausgang}

Der Antragsteller kann gegen den Genehmigungsbescheid - wie gegen jeden anderen Verwaltungsakt auch - Rechtsmittel einlegen. Der Widerspruch, der im einzelnen materiell zu begründen ist, ${ }^{273}$ wird zunächst von der Behörde, die den angegriffenen Verwaltungsakt erlassen hat, im vorliegenden Fall also von der Genehmigungsbehörde, i.S. einer Eigenkontrolle auf Möglichkeiten einer Abhilfe der vorgetragenen Einwendungen des Widerspruchsführers hin geprüft. Hält die Behörde die Einwendungen für zulässig und begründet, erläßt sie einen entsprechenden (kostenfreien) Abhilfebescheid. Sieht sie sich hierzu nicht in der Lage, leitet sie den Widerspruch zusammen mit einem verwaltungsinternen Vorlagebericht über das (negative) Ergebnis ihrer Abhilfeprüfung der (übergeordneten) Widerspruchsbehörde (hier die niedersächsische Bezirksregierung bzw. das baden-württembergische Regierungspräsidium) zu, die das Widerspruchsverfahren in Form eines Widerspruchsbescheids abschließend entscheidet. Der Betreiber trägt die Kosten des Verfahrens, soweit seinem Widerspruch nicht stattgegeben wird. Gegen den Widerspruch kann er Klage beim Verwaltungsgericht erheben.

Soweit der formelle Gang des verwaltungsförmigen Widerspruchsverfahrens. In der Praxis ist es dagegen weit verbreitet (und war auch in den hier untersuchten einschlägigen Fällen zu beobachten), daß die Behörde den Widerspruchsführer vorab informiert, wenn sie keine Abhilfemöglichkeit sieht, und ihn auffordert mitzuteilen, ob er angesichts dieser Lage seinen Widerspruch aufrechterhalten (und das Risiko einer kostenpflichtigen Ablehnung durch die Widerspruchsbehörde eingehen) will. Diese »War-

273 Eine rechtlich-juristische Begründung ist dagegen nicht erforderlich. Im übrigen müssen die Einwendungen gegen den Verwaltungsakt nicht explizit als Widerspruch bezeichnet werden. Es genügt, wenn der Betroffene zum Ausdruck bringt, in welchen Punkten er sich gegen die behördliche Entscheidung zur Wehr setzen will. Abgrenzungsprobleme in dieser Hinsicht traten in der vorliegenden Untersuchung allerdings nicht auf. 
nung « ist freilich nicht ganz uneigennützig, da die Behörde im Falle der Weiterleitung des Widerspruchs ihrerseits Gefahr läuft, zu unterliegen.

Dem behördlichen Interesse, förmliche Widerspruchsverfahren möglichst zu verhindern, ist - wie bereits angesprochen (Ziff. 3.2) - auch die beim GAA-N geübte Praxis der Zuleitung eines Bescheidsentwurfs zuzurechnen. Der Antragsteller erhält so Gelegenheit, durch Einwendungen auf eine »Revision« der Genehmigungsbestimmungen zu seinen Gunsten hinzuwirken. Von daher läßt sich das Instrument des Vorabentwurfs als funktionales Äquivalent zum förmlichen Widerspruch betrachten. Der entscheidende Unterschied zwischen den beiden Varianten der »Gegenwehr « des Betreibers ist im wesentlichen in den Folgen für die Genehmigungsbehörde als Adressatin zu sehen. Ihr ist die Reaktion auf Einwendungen gegen den Entwurf freigestellt, da es sich jeweils um bloße inoffizielle, nur der Eigenkontrolle unterliegende Instrumente handelt. Dies bedeutet insbesondere, daß sie für ihre Entscheidung, ob bzw. wieweit sie der Beschwerde nachgibt, weder dem Betreiber noch der vorgesetzten Instanz Rechenschaft schuldig ist. ${ }^{274}$ Beim förmlichen Widerspruch unterliegt die Behörde dagegen - soweit sie keine vollständige Abhilfe schafft - einer Begründungspflicht und muß sich der »externen« Kontrolle durch die Widerspruchsbehörde aussetzen. Mit dieser »Fremdkontrolle« verbindet sich das Risiko aktenkundiger Abänderungen der Genehmigungsentscheidung, was den künftigen Handlungsspielraum der Genehmigungsbehörde in ähnlich gelagerten Fällen einschränkt. Für diese ist es von daher eine naheliegende Strategie, jenes Risiko möglichst zu minimieren, etwa eben durch das bereits näher beschriebene Einräumen vorgelagerter, inoffizieller Beschwerdemöglichkeiten gegen einen als »probeweise « deklarierten Genehmigungsbescheid. Die Frage, wieweit dieses Mittel tatsächlich trägt, förmliche Widersprüche »aufzufangen«, läßt sich überprüfen, wenn man die Einwendungen gegen Vorabentwürfe als eine Art faktischer Widersprüche bei der nachfolgenden quantitativen und qualitativen Bilanz der (förmlichen) Widersprüche mit in Rechnung stellt.

\subsubsection{Häufigkeit von Widersprüchen}

Einen Überblick über die Häufigkeit förmlicher Widersprüche gegen den Genehmigungsbescheid in den beiden Modellen gibt Tab. 33. Zum Vergleich ist der jeweilige Gesamtanteil an Einwänden gegen Genehmigungsauflagen ausgewiesen, der sich aus den förmlichen Widersprüchen und den gegen Entwurfsfassungen vorgebrachten Einwendungen i.S. faktischer Widersprüche zusammensetzt (Zeilen im Kleindruck).

274 Die Freistellung von einer expliziten Rechtfertigungspflicht bedeutet selbstverständlich keine Entbindung vom rechtlich Zulässigen. 
Tab. 33

Häufigkeit förmlicher Widersprüche gegen den Genehmigungsbescheid

\begin{tabular}{|l|c|c|c|}
\cline { 2 - 4 } \multicolumn{1}{c|}{} & GAA-N & $L R A-1$ & $L R A-2$ \\
\hline \multirow{2}{*}{ Förmlicher Widerspruch } & 15 & 6 & 1 \\
[Förmliche und faktische Widersprüche] & $25,9 \%$ & $14,0 \%$ & $4,2 \%$ \\
& {$[26]$} & {$[8]$} & {$[1]$} \\
\multirow{2}{*}{ Kein förmlicher Widerspruch } & {$[44,8 \%]$} & {$[18,6 \%]$} & {$[4,2 \%]$} \\
\multirow{2}{*}{ Kein förmlicher oder faktischer Widerspruch] } & {$[53,2 \%]$} & 37 & 23 \\
\hline \hline \multirow{2}{*}{ Verfahren gesamt } & $74,1 \%$ & $86,0 \%$ & $95,8 \%$ \\
& 58 & {$[35]$} & {$[23]$} \\
& $100,0 \%$ & $100,0 \%$ & $100,0 \%$ \\
\hline
\end{tabular}

Betrachtet man zunächst die Gruppe der förmlichen Widersprüche, so wurden sie in rd. einem Viertel $(25,9 \%)$ der Verfahren beim GAA-N, aber nur in rd. einem Siebtel $(14,0 \%)$ der Verfahren beim LRA-1 erhoben. ${ }^{275}$ Der Unterschied bleibt aber unterhalb statistischer Signifikanz. ${ }^{276}$

Das Bild ändert sich, wenn man auch die faktischen Einwendungen mit einbezieht (Zeilen im Kleindruck). Führte dieses dem Antragsteller in erster Linie beim GAA-N zusätzlich verfügbare - vom förmlichen Widerspruch grundsätzlich unabhängige - Instrument dazu, daß fast jeder zweite $(44,8 \%)$ Genehmigungsbescheid bzw. dessen Entwurf bemängelt wurde, erhöht sich der vergleichbare Anteil beim LRA-1, das Vorabentwürfe lediglich ausnahmsweise überstellte, nur geringfügig auf $18,6 \%$, was etwa jedem fünften Verfahren entspricht. ${ }^{277}$ Dieses Gefälle ist statistisch signifikant. ${ }^{278}$

Das Ergebnis belegt in anderer Form noch einmal das schon weiter oben (Ziff. 3.2) festgestellte Potential von Entwurfsfassungen, förmliche Widerspruchsverfahren zu verhindern. Auf der anderen Seite bleibt aber auch festzuhalten, daß insgesamt das Niveau der Widerspruchsneigung beim GAA-N immer noch deutlich - wenngleich nicht signifikant - über dem beim LRA-1 trotz dessen weitgehendem Verzicht auf Vorabentwürfe liegt bzw. dieses keinesfalls unterschreitet. Das führt zu der ergänzenden These, daß sich die prophylaktische Wirkung des Genehmigungsentwurfs im Er-

275 Beim LRA-2 wurde in nur einem Fall Widerspruch eingelegt.

$276 \mathrm{chi}^{2}=2,13<2,71$ für $\mathrm{p}=0,1$ und $\mathrm{df}=1$.

277 Beim LRA-1 wurde, wie erwähnt, nur in zwei Fällen dem Antragsteller auf dessen ausdrücklichen Wunsch eine Entwurfsfassung überstellt. In beiden Fällen machte dieser Einwendungen geltend (vgl. oben Anm. 242).

$278 \mathrm{chi}^{2}=7,61>6,64, \mathrm{p}=0,01, \mathrm{df}=1$. 
gebnis darin erschöpft, den von diesem selbst evozierten Effekt i.S. einer Ermunterung zur Gegenwehr zu neutralisieren. ${ }^{279}$

Im Rahmen der vorliegenden Untersuchung ist die weitere Frage von besonderem Interesse, ob bzw. inwieweit die unterschiedliche Widerspruchsneigung der Antragsteller sich in beiden Genehmigungsmodellen gleichermaßen auf immissionsschutzrechtliche und sonstige Belange betreffende Auflagen erstreckt. Die Ergebnisse sind in Tab. 34.1 für die gegen immissionsschutzrechtliche Bestimmungen und in Tab. 34.2 für die gegen sonstige Bestimmungen gerichteten Widersprüche dargestellt. Analog zu Tab. 33 sind auch hier zusätzlich die jeweiligen Gesamtanteile an förmlichen und faktischen Einwendungen ausgewiesen (Zeilen im Kleindruck). ${ }^{280}$

Was die förmlichen Widersprüche gegen immissionsschutzrechtliche Auflagen anbelangt (Tab. 34.1), so ergibt sich auch für diese Teilmenge beim GAA-N ein höherer Anteil als beim LRA-1 (13,8\% gegenüber 7,0\%). Wie bei der Gesamtmenge der Widersprüche bleibt aber die Differenz unterhalb statistischer Signifikanz. ${ }^{281}$ Die Angleichung der formellen Widerspruchshäufigkeiten verdankt sich auch hier der Absorption von Gegenwehr durch Vorabentwürfe. Nimmt man die gegen diese vorgetragenen Einwendungen hinzu (Zeilen im Kleindruck), ist beim GAA-N ein deutlicher Anstieg des Revisionsverlangens der Genehmigungsentscheidung auf ein knappes Viertel $(24,1 \%)$ der Verfahren zu verzeichnen, dem lediglich ein knappes Zehntel $(9,3 \%)$ beim LRA-1 gegenübersteht. Dieser Unterschied ist wiederum statistisch signifikant. ${ }^{282}$

Betrachtet man nun die Widersprüche gegen sonstige, nicht-immissionsschutzrechtliche Belange abdeckende Auflagen (Tab. 34.2), sticht als erstes hervor, daß sie in beiden Genehmigungsmodellen diejenigen gegen immissionsschutzrechtliche Auflagen (Tab. 34.1) übertreffen. In statistisch signifikantem Ausmaß ist dies allerdings nur beim GAA-N der Fall und dort zu-

279 Die Aussage ist im statistischen Sinne zu verstehen, d.h. sie gilt nicht unbedingt auch im jeweiligen konkreten Einzelfall.

280 In drei Fällen - alle beim GAA-N - wurden sowohl Einwendungen gegen die Entwurfsfassung als auch förmlicher Widerspruch gegen den abschließenden, den vorgebrachten Monita gegen den Entwurf nicht bzw. nur teilweise Rechnung tragenden Genehmigungsbescheid erhoben. Diese Kumulation von faktischem und nachfolgendem förmlichen Widerspruch in ein und demselben Verfahren schlägt sich zwangsläufig nicht in der Häufigkeit der beide Formen umfassenden Hauptkategorie der Tabelle nieder.

$281 \mathrm{chi}^{2}=1,18<2,71$ für $\mathrm{p}=0,1$ und $\mathrm{df}=1$.

$282 \mathrm{chi}^{2}=3,71>2,71, \mathrm{p}=0,1, \mathrm{df}=1$. Der Testwert verfehlt knapp das nächsthöhere Signifikanzniveau von $\mathrm{p}=0,05$ für $\mathrm{chi}^{2}>3,84$ und $\mathrm{df}=1$. 
dem nur bei Berücksichtigung auch der nicht-förmlichen Einwendungen (Zeilen im Kleindruck). ${ }^{283}$

\section{Tab. 34.1}

Häufigkeit förmlicher Widersprüche gegen immissionsschutzrechtliche Auflagen des Genehmigungsbescheids

\begin{tabular}{|l|c|c|c|}
\cline { 2 - 4 } \multicolumn{1}{c|}{} & GAA-N & LRA-I & LRA-2 \\
\hline \multirow{2}{*}{ Förmlicher Widerspruch } & 8 & 3 & 1 \\
[Förmliche und faktische Widersprüche $]$ & $13,8 \%$ & $7,0 \%$ & $4,2 \%$ \\
& {$[14]$} & {$[4]$} & {$[1]$} \\
Kein förmlicher Widerspruch & {$[24,1 \%]$} & {$[9,3 \%]$} & {$[4,2 \%]$} \\
\multirow{2}{*}{ [Kein förmlicher oder faktischer Widerspruch] } & {$[75,9 \%]$} & {$[90,7 \%]$} & {$[95,8 \%]$} \\
\hline \hline \multirow{2}{*}{ Verfahren gesamt } & $56,2 \%$ & $93,0 \%$ & $95,8 \%$ \\
& 58 & 43 & 24 \\
\hline
\end{tabular}

Im einzelnen ist für die nicht-immissionsschutzrechtlichen Auflagen beim GAA-N ein signifikant höherer Anteil als beim LRA-1 bereits allein an förmlichen Widersprüchen zu verbuchen $(25,9 \%$ gegenüber $9,3 \%),{ }^{284}$ was im immissionsschutzrechtlichen Bereich nicht der Fall war. Auf der anderen Seite ähnelt sich die Situation bei beiden Auflagenarten wiederum insoweit, als auch hier die Zahl der förmlichen Widersprüche, insbesondere beim GAA-N, Ergebnis erfolgreicher Reduktion eines anfänglich höheren (faktischen) Widerspruchspotentials ist. Das zeigt sich im deutlich größeren Gefälle des Gesamtanteils an förmlichen und nicht-förmlichen Revisionsbegehren, der sich in Niedersachsen auf $41,4 \%$ der Verfahren, beim LRA-1 dagegen lediglich auf $14,0 \%$ beläuft. ${ }^{285}$

$283 \mathrm{chi}^{2}=3,91>3,84, \mathrm{p}=0,05, \mathrm{df}=1$. Bezieht man den Vergleich der Widerspruchshäufigkeiten gegen immissionsschutzrechtliche Auflagen einerseits und sonstige Auflagen andererseits ausschließlich auf die förmlichen Widersprüche, bleibt der Testwert knapp unter der Signifikanzgrenze $\left(\mathrm{chi}^{2}=2,66<2,71\right.$ für $\mathrm{p}=0,1$ und df $\left.=1\right)$. $284 \mathrm{chi}^{2}=4,44>3,84, \mathrm{p}=0,05$, df $=1$.

285 Das spiegelt sich auch im Signifikanzniveau wider: chi $^{2}=9,89>7,88, p=0,005$, $\mathrm{df}=1$. 
Tab. 34.2

Häufigkeit förmlicher Widersprüche gegen sonstige, nicht-immissionsschutzrechtliche Auflagen des Genehmigungsbescheids

\begin{tabular}{|l|c|c|c|}
\cline { 2 - 4 } \multicolumn{1}{c|}{} & $G A A-N$ & $L R A-1$ & $L R A-2$ \\
\hline Förmlicher Widerspruch & 15 & 4 & 1 \\
& $25,9 \%$ & $9,3 \%$ & $4,2 \%$ \\
[Förmliche und faktische Widersprüche] & {$[24]$} & {$[6]$} & {$[1]$} \\
& {$[41,4 \%]$} & {$[14,0 \%]$} & {$[4,2 \%]$} \\
\hline \multirow{2}{*}{ Kein förmlicher Widerspruch } & 43 & 39 & 23 \\
[Kein förmlicher oder faktischer Widerspruch] & {$[5,1 \%$} & $90,7 \%$ & $95,8 \%$ \\
\hline \hline \multirow{2}{*}{ Verfahren gesamt } & {$[34]$} & {$[37]$} & {$[23]$} \\
& 58 & {$[86,0 \%]$} & {$[95,8 \%]$} \\
\hline
\end{tabular}

Die Verringerung der Differenz an Widersprüchen zwischen den beiden Genehmigungsmodellen bei Ausblendung der nicht-förmlichen Einwendungen, wie sie auch bei den immissionsschutzrechtlichen Auflagen zu beobachten war, läßt sich wie dort auf den förmliche Einreden auffangenden Mechanismus des Vorabentwurfs zurückführen. Das hier gleichwohl verbleibende Nord-Süd-Gefälle in der Widerspruchsneigung in nicht-immissionsschutzrechtlichen Belangen ist damit aber noch nicht erklärt.

Als ausschlaggebenden Einflußfaktor einschlägige Streitigkeiten im vorausliegenden Verfahrensverlauf zu vermuten, liegt nahe. Tatsächlich war es jedoch, wie dargelegt (vgl. oben Ziff. 3.1.3), beim GAA-N zum einen nicht öfter als beim LRA-1 zu Kontroversen über nicht-immissionsschutzrechtliche Belange gekommen. Unabhängig davon besteht zum anderen bei beiden Genehmigungsbehörden kein statistischer Zusammenhang zwischen vorausgegangenen Verfahrenskonflikten und förmlichen Widersprüchen. ${ }^{286}$ Ein solcher ist dagegen, wie an anderer Stelle bereits erwähnt, beim GAA-N zwischen Bescheidsentwurf und Verzicht auf förmlichen Widerspruch gegeben. ${ }^{287}$ Von den 15 Widersprüchen erfolgten 12 in Verfahren, in denen der Antragsteller keine Entwurfsfassung erhalten hatte, und entsprechend nur drei, in denen das der Fall war. Bei den immissionsschutzrechtliche Auflagen betreffenden Widersprüchen besteht ein solcher statis-

286 Der entsprechende Testwert liegt beim GAA-N nur bei chi $^{2}=0,03<2,71$ für $p=$ 0,1 und $\mathrm{df}=1$. Beim LRA- 1 ist ein etwaiger Zusammenhang ebenfalls auszuschließen, wegen der geringen (absoluten) Zahl von lediglich vier Widersprüchen aber statistisch nicht mehr nachweisbar.

287 Vgl. oben Ziff. 3.2 mit Anm. 238. 
tischer Konnex nicht. Hinzu kommt, daß die immissionsschutzrechtlichen Streitigkeiten häufiger als die sonstigen Konflikte noch im laufenden Verfahren zur Zufriedenheit des Antragstellers geregelt wurden, wofür der geringere Anteil an Änderungswünschen der entsprechenden Auflagen in den Entwurfsfassungen spricht (vgl. oben Ziff. 3.2, Tab. 28). ${ }^{288}$

Mit Blick auf diese verschiedenen Zusammenhänge bietet sich als Erklärung für die höheren Anteile an förmlichen nicht-immissionsschutzrechtlichen Widersprüchen in Niedersachsen gegenüber Baden-Württemberg folgende These an. Der Antragsteller macht bei der niedersächsischen Genehmigungsbehörde die Erfahrung, daß es sich lohnt, um möglichst günstige Genehmigungsbedingungen zu kämpfen. Erfolge kann er dabei insbesondere bei den immissionsschutzrechtlichen Belangen verbuchen, da diese der (im rechtlichen Rahmen) autonomen Disposition des GAA-N als Fachund Genehmigungsinstanz unterliegen. Entsprechend ist er geneigt, diese Erfahrung zu generalisieren und bei den übrigen beteiligten Fachbehörden ebenfalls auf ein Entgegenkommen zu setzen. Soweit der Verfahrensverlauf selbst keine Gelegenheit zum »Aushandeln« geboten hat, sei es, weil die betroffene Fachbehörde nicht unmittelbar am Verfahren beteiligt war, sei es, weil der Antragsteller nicht durch einen Vorabentwurf über deren Auflagen informiert wurde, bietet sich ihm der förmliche Widerspruch als (letztes) Mittel zur Wahrung seiner Interessen an. Daß dabei seine Aussichten denen bei nicht-förmlichen Einwendungen nicht nachstehen, zeigt der nachfolgende Abschnitt.

Demgegenüber mußte der Antragsteller bei der baden-württembergischen Genehmigungsbehörde häufig erleben, daß selbst beharrliches Verhandeln unter Fürsprache der Genehmigungsbehörde das GAA-1 nur begrenzt dazu bewegen konnte, von seinen einmal eingenommenen Positionen abzurücken und sich auf Konzessionen einzulassen. Von daher dürfte sich der Betreiber insgesamt weniger ermutigt sehen, darauf zu setzen, mit einem Widerspruch den Behörden mehr als das bisher Erreichte abhandeln zu können.

\subsubsection{Ausgang der Widerspruchsverfahren (Entscheidung der Genehmigungsbehörde)}

Die förmlichen Widersprüche lassen sich aus Sicht des Antragstellers als das im Verfahren selbst nicht zufriedenstellend bewältigte oder auch nicht offen zur Sprache gebrachte Konfliktpotential betrachten. Die Bedingungen, denen die nachträgliche Interessenwahrung im Wege eines offiziellen

288 Die (auf geringen absoluten Häufigkeiten beruhende) Differenz bleibt allerdings unterhalb des Signifikanzniveaus. 
Widerspruchsverfahrens unterliegen, erscheinen auf den ersten Blick wenig günstig. Schließlich haben sich die Genehmigungsbehörde bzw. die faktisch weitgehend autonom agierenden Fachbehörden mit ihren Anforderungen in dem erlassenen Genehmigungsbescheid rechtsverbindlich festgelegt. Ein Abrücken von der getroffenen Entscheidung ist zwangsläufig damit verbunden, eine Fehlentscheidung oder einen Irrtum eingestehen zu müssen. Auf der anderen Seite erwächst dem Betreiber - unter den Bedingungen der oben dargelegten (Ziff. 3.4) gängigen Verwaltungspraxis - auch im förmlichen Widerspruchsverfahren erst dann ein (Kosten-) Risiko, wenn er bei negativem Ausgang der vorgeschalteten Abhilfeprüfung durch die Genehmigungsbehörde an seinem Widerspruch festhält.

Der Ausgang der Widerspruchsverfahren wird in der vorliegenden Untersuchung nur bis zur Entscheidung der Genehmigungsbehörde in Form der Abhilfeprüfung verfolgt. Dazu gehört auch die Reaktion des Widerspruchsführers (Antragstellers) im Sinne der Akzeptanz bzw. (durch Aufrechterhaltung des Widerspruchs dokumentierte) Nichtakzeptanz der getroffenen Entscheidung in denjenigen Fällen, in denen die Genehmigungsbehörde keine vollständige Abhilfe geschaffen hat. ${ }^{289}$

Die Befunde zur behördlichen Abhilfeprüfung sind in Tab. 35.1 im Hinblick auf die Einwendungen gegen immissionsschutzrechtliche Auflagen und in Tab. 35.2 im Hinblick auf die Einwendungen gegen sonstige, nichtimmissionsschutzrechtliche Auflagen wiedergegeben. Einschränkend ist allerdings hinzuzufügen, daß die geringe (absolute) Anzahl an förmlichen Widersprüchen, namentlich beim LRA-1, es nicht zuläßt, die Häufigkeitsverteilungen der erfolgten Abhilfe mit statistischen Mitteln vergleichend zu analysieren und die Ergebnisse entsprechend abzusichern. Systematische Unterschiede zwischen der niedersächsischen und der baden-württembergischen Genehmigungsbehörde bzw. den zuständigen Fachämtern lassen sich von daher nicht identifizieren. Insgesamt sprechen die Befunde eher für eine elementare Ähnlichkeit der beteiligten Ämter in der Reaktion auf förmliche Widersprüche. Bei beiden überwiegt die Bereitschaft zur (Selbst-) Korrektur der angegriffenen Auflagen der Genehmigungsentscheidung. Das war angesichts des damit verbundenen Risikos eines Autoritäts- und Glaubwürdigkeitsverlusts gegenüber dem Betreiber nicht ohne

289 Den Ausgang der Abhilfeprüfung durch die Genehmigungsbehörde über den Widerspruch (einschließlich der Reaktion des Antragstellers) hier als Maßstab für die Wirksamkeit dieses Mittels zugrunde zu legen, entspricht der Konzentration der Untersuchung auf das jeweilige Entscheidungsverhalten der Genehmigungsbehörde in den beiden Organisationsmodellen. Die (übergeordnete) Widerspruchsbehörde und entsprechend deren Entscheidung bilden eine von der (unteren) Genehmigungsbehörde organisatorisch separierte, unabhängige Ebene der Verwaltung. Abgesehen davon hatte die (übergeordnete) Widerspruchsbehörde die (insgesamt wenigen) ihr vorgelegten (aufrechterhaltenen) Widersprüche bei Abschluß der Erhebungsphase - von einer Ausnahme abgesehen - noch nicht beschieden. 
weiteres zu erwarten. Schwerer als dies wiegt aber offenbar das behördliche Interesse daran, eine Vorlage des Widerspruchs bei der oberen Widerspruchsbehörde möglichst abzuwehren.

Wendet man sich zunächst der (kleineren) Anzahl der förmlichen Widersprüche gegen immissionsschutzrechtliche Auflagen zu, ergibt sich für Umfang und Ausmaß der behördlichen Abhilfe folgendes Bild (Tab. 35.1).

Tab. 35.1

Ausgang förmlicher Widersprüche gegen immissionsschutzrechtliche Auflagen des Genehmigungsbescheids*

\begin{tabular}{|l|c|c|c|}
\cline { 2 - 4 } \multicolumn{1}{c|}{} & $G A A-N$ & $L R A-I$ & $L R A-2$ \\
\hline Vollständige Abhilfe & 2 & 0 & 1 \\
[Förmliche und faktische Widersprüche] & {$[35,0 \%$} & $0,0 \%$ & $100,0 \%$ \\
& {$[5]$} & {$[0]$} & {$[1]$} \\
Teilweise Abhilfe & 5 & {$[0,0 \%]$} & {$[100,0 \%]$} \\
\hline & $62,5 \%$ & 1 & 0 \\
[Förmliche und faktische Widersprüche] & {$[35]$} & $33,3 \%$ & $0,0 \%$ \\
& 1 & {$[1]$} & {$[0]$} \\
Keine Abhilfe & $12,5 \%$ & $625,0 \%]$ & {$[0,0 \%]$} \\
[Förmliche und faktische Widersprüche] & {$[28,6 \%]$} & {$[75,0 \%]$} & {$[0,0 \%]$} \\
\hline \hline Förmliche Widersprüche gesamt & 8 & 3 & 0 \\
[Förmliche und faktische Widersprüche] & {$[100,0 \%]$} & {$[100,0 \%]$} & {$[100,0 \%]$} \\
\hline
\end{tabular}

* Angesichts der niedrigen absoluten Grundgesamtheiten an Widersprüchen dient die Prozentuierung lediglich einer näherungsweisen (heuristischen) Vergleichbarkeit der auf die einzelnen Kategorien entfallenden absoluten Häufigkeiten.

Von den insgesamt acht förmlichen Widerspruchsfällen beim GAA-N gingen fünf zumindest teilweise und zwei vollständig zugunsten des Antragstellers aus. ${ }^{290}$ Lediglich in einem Fall hielt die Behörde an ihrer ursprüng-

290 Die häufigsten Widerspruchspunkte betrafen Meßvorgaben für Emissions- bzw. Immissionsnachweise und bauliche Maßnahmen zur Minderung von Schadstoffemissionen. Einreden gegen Grenzwertfestsetzungen blieben dagegen die Ausnahme. 
lichen Entscheidung fest. ${ }^{291}$ Zum Vergleich sind - analog zu Tab. 33, 34.1 und 34.2 - die Anteile in den drei Abhilfekategorien für die Gesamtheit der förmlichen und nicht-förmlichen Einwendungen ausgewiesen (Zeilen im Kleindruck). Dabei zeigt sich - was vielleicht so nicht zu erwarten war -, daß nicht-förmlichen Revisionsbegehren öfter als förmlichen jegliches (abhelfendes) Entgegenkommen verweigert wurde. ${ }^{292}$ Der Grund könnte darin zu suchen sein, daß sich die Folgen gänzlich verweigerter Abhilfe eines förmlichen Widerspruchs der Kontrolle der Genehmigungsbehörde entziehen. ${ }^{293}$

Beim LRA-1 nahmen die förmlichen Widersprüche wie auch die einzige nicht-förmliche Einwendung einen eher ungünstigen Ausgang für den Antragsteller mit nur einer teilweisen Abhilfe und zwei bzw. bei Einrechnung der nicht-förmlichen Einwendungen insgesamt drei vollständigen Zurückweisungen. ${ }^{294}$ Die geringe Anzahl von lediglich drei bzw. vier einschlägigen Fällen erlaubt, wie gesagt, nicht, aus den Befunden weitergehende (vergleichende) Schlußfolgerungen zu ziehen.

Insgesamt erfolgreicher waren die (zahlreicheren) förmlichen Widersprüche gegen sonstige, nicht-immissionsschutzrechtliche Genehmigungs-

291 Der Antragsteller, ein mittelgroßes Unternehmen, erhielt seinen Widerspruch aufrecht. Bei Abschluß der Erhebung lag noch kein Bescheid der Widerspruchsbehörde (Bezirksregierung) vor.

292 Dabei ist daran zu erinnern, daß die Antragsteller auf die in vollem Umfang zurückgewiesenen nicht-förmlichen Einwendungen (drei Fälle) nicht mit einem förmlichen Widerspruchsverfahren reagiert haben (Ziff. 3.2, nach Tab. 29). Bei den insgesamt verweigerten (förmlichen) Abhilfen handelt es sich also um davon verschiedene, nichtidentische Fälle.

293 Das gilt im Prinzip auch für lediglich teilweise geschaffene Abhilfe. Im Unterschied zur vollständigen Ablehnung läuft in diesem Fall der Antragsteller bei Aufrechterhaltung seines Widerspruchs allerdings Gefahr, daß die (übergeordnete) Widerspruchsbehörde nicht nur die von der Genehmigungsinstanz verweigerte Abhilfe bestätigt, sondern auch die von dieser eingeräumten Zugeständnisse wieder zurücknimmt (»reformatio in peius $«$ ).

294 In den beiden Fällen nicht abgeholfener (förmlicher) Widersprüche hielten (wie beim GAA-N) die Antragsteller (jeweils ein Großunternehmen) an diesen fest. Dem LRA-1 war allerdings beide Male eine Abhilfe insofern von vornherein verwehrt, als es an einen die angegriffene Auflage als zwingend vorschreibenden Erlaß des Ministeriums gebunden war. Bei Abschluß der Erhebung lag ein Bescheid der Widerspruchsbehörde (Regierungspräsidium) noch nicht vor. Auch im Fall des lediglich teilweise abgeholfenen (förmlichen) Widerspruchs insistierte der Antragsteller, ein Großunternehmen, auf seiner Einrede. Der Bescheid der Widerspruchsbehörde (Regierungspräsidium) enthält einen »Kompromiß« zwischen der Forderung des Betreibers und der (nur bedingt) gewährten Abhilfe der Genehmigungsbehörde. Im (einzigen) Fall der vollständig zurückgewiesenen nicht-förmlichen Einwendung hat der Betreiber die Entscheidung der Genehmigungsbehörde dagegen akzeptiert (vgl. oben Anm. 242). 
auflagen (Tab. 35.2) ${ }^{295}$ Beim GAA-N wurde in zwei Dritteln $(66,7 \%)$ der Fälle vollständige Abhilfe geschaffen, lediglich in einem Fall blieb es bei der ursprünglichen Entscheidung. Dabei lag die Entscheidung über eine mögliche Abhilfe - wie zuvor bei den nicht-förmlichen Einwendungen (vgl. oben Ziff. 3.2) - faktisch in der Hand der betroffenen Fachämter. Seine entsprechende Anfrage hat das GAA-N in den meisten Fällen mit der Befürwortung einer wohlwollenden Prüfung verbunden. Damit blieb es seiner schon im Verfahren verfolgten Linie treu, in Streitigkeiten zwischen Fachbehörde und Antragsteller zugunsten des Antragstellers zu intervenieren. ${ }^{296}$ Diese Strategie ermöglicht es der niedersächsischen Behörde auf der einen Seite, sich gegenüber dem Antragsteller als dessen Anliegen unterstützende Instanz zu präsentieren. Auf der anderen Seite bereitet sie damit den Boden für eine höhere Abnahmebereitschaft des Antragstellers hinsichtlich der immissionsschutzrechtlich gebotenen, von ihr selbst (fachlich) zu verantwortenden Genehmigungsanforderungen.

Auch beim LRA-1 war den Widersprüchen gegen nicht-immissionsschutzrechtliche Auflagen größerer Erfolg beschieden als denen gegen immissionsschutzrechtliche Auflagen. Den drei förmlichen Widersprüchen und der einzigen nicht-förmlichen Einwendung wurde je zur Hälfte vollständig bzw. zumindest teilweise stattgegeben. Allerdings leidet auch hier die Möglichkeit eines aussagekräftigen Vergleichs sowohl intern zwischen den auflagenbezogenen Widerspruchsarten als auch extern mit dem GAA$\mathrm{N}$ an der geringen absoluten Fallzahl.

295 Die häufigsten Widerspruchspunkte betrafen den Arbeitsschutz sowie - mit deutlichem Abstand - den Brandschutz. In dem Umstand, daß auch die fachliche Zuständigkeit für den Arbeitsschutz beim GAA-N liegt, könnte eine maßgebliche Ursache für die vergleichsweise hohe Zahl von Einreden speziell gegen diese Auflagenart zu suchen sein. Die Erfahrung einer gewissen Verhandlungsbereitschaft des GAA$\mathrm{N}$ mag den Antragsteller veranlaßt haben, auch auf diesem Gebiet auf ein größeres behördliches Entgegenkommen als bei anderen Fachämtern zu setzen. Beim LRA1 war in keinem Fall eine arbeitsschutzrechtliche Bestimmung moniert worden, die ebenfalls in die fachliche Zuständigkeit des dortigen, aber weniger nachgiebig agierenden GAA-1 fällt.

296 Vg1. oben Ziff. 3.1.3. mit Tab. 25.1. 
Tab. 35.2

Ausgang förmlicher Widersprüche gegen sonstige, nicht-immissionsschutzrechtliche Auflagen des Genehmigungsbescheids*

\begin{tabular}{|c|c|c|c|}
\hline & \\
\hline & $G A A-N$ & LRA-1 & $L R A-2$ \\
\hline $\begin{array}{l}\text { Vollständige Abhilfe } \\
\text { [Förmliche und faktische Widersprüche] }\end{array}$ & $\begin{array}{c}10 \\
66,7 \% \\
{[13]} \\
{[54,2 \%]}\end{array}$ & $\begin{array}{c}2 \\
50,0 \% \\
{[3]} \\
{[50,0 \%]}\end{array}$ & $\begin{array}{c}1 \\
100,0 \% \\
{[1]} \\
{[100,0 \%]}\end{array}$ \\
\hline $\begin{array}{l}\text { Teilweise Abhilfe } \\
\text { [Förmliche und faktische Widersprüche] }\end{array}$ & $\begin{array}{c}4 \\
26,7 \% \\
{[8]} \\
{[33,3 \%]}\end{array}$ & $\begin{array}{c}2 \\
50,0 \% \\
{[3]} \\
{[50,0 \%]}\end{array}$ & $\begin{array}{c}0 \\
0,0 \% \\
{[0]} \\
{[0,0 \%]}\end{array}$ \\
\hline $\begin{array}{l}\text { Keine Abhilfe } \\
\text { [Förmliche und faktische Widersprüche] }\end{array}$ & $\begin{array}{c}1 \\
6,7 \% \\
{[3]} \\
{[12,5 \%]}\end{array}$ & $\begin{array}{c}0 \\
0,0 \% \\
{[0]} \\
{[0,0 \%]}\end{array}$ & $\begin{array}{c}0 \\
0,0 \% \\
{[0]} \\
{[0,0 \%]}\end{array}$ \\
\hline $\begin{array}{l}\text { Förmliche Widersprüche gesamt } \\
\text { [Förmliche und faktische Widersprüche] }\end{array}$ & $\begin{array}{c}15 \\
100,1 \% \\
{[24]} \\
{[100,0 \%]}\end{array}$ & $\begin{array}{c}4 \\
100,0 \% \\
{[6]} \\
{[100,0 \%]}\end{array}$ & $\begin{array}{c}1 \\
100,0 \% \\
{[1]} \\
{[100,0 \%]}\end{array}$ \\
\hline
\end{tabular}

* Angesichts der niedrigen absoluten Grundgesamtheiten an Widersprüchen dient die Prozentuierung lediglich einer näherungsweisen (heuristischen) Vergleichbarkeit der auf die einzelnen Kategorien entfallenden absoluten Häufigkeiten.

Insgesamt überwiegen in der Behandlung förmlicher Widersprüche die Ähnlichkeiten zwischen den beiden Genehmigungsmodellen. Im Norden wie im Süden lohnte es sich fast immer, sich mit diesem Instrument gegen Genehmigungsauflagen, immissionsschutzrechtlicher und (noch mehr) sonstiger Art, ${ }^{297}$ zur Wehr zu setzen. Das einzige, was die Modelle trennt, betrifft das quantitative Aufkommen an Widersprüchen. In dieser Hinsicht scheint die niedersächsische Konzeption es dem Antragsteller leichter zu machen, diese Möglichkeit für sich in Anspruch zu nehmen.

297 Der vergleichsweise größere Erfolg (förmlicher) Widersprüche gegen nicht-immissionsschutzrechtliche Auflagen spiegelt sich auch darin, daß alle aufrechterhaltenen (förmlichen) Widersprüche in beiden Modellen ausnahmslos nur immissionsschutzrechtlichen Auflagen galten. 


\section{Verfahrensdauer(n)}

\subsection{Die Verfahrensdauer als Gegenstand von Kritik und Gesetzesreformen}

Die Dauer von Genehmigungsverfahren nimmt seit längerem einen herausragenden, wenn nicht führenden Platz in der Kritik am Verwaltungshandeln ein. Die dafür stehende Debatte zur Beschleunigung von Planungs- und Genehmigungsverfahren hat maßgebliche Impulse aus den beklagten wirtschaftlichen Nachteilen erhalten, die aus der investitionshemmenden Wirkung überlanger Verfahrensdauern für den »Wirtschaftsstandort Deutschland « erwüchsen. ${ }^{298}$

Angesichts dieser Kritik und der darin enthaltenen Aufforderung, auf eine Verkürzung der Genehmigungsverfahren hinzuwirken, führte der Gesetzgeber mit Wirkung ab Mai 1993 Regelfristen für die behördliche Bearbeitungsdauer von Genehmigungsanträgen ein. ${ }^{299}$ Der in $\S 10 \mathrm{BImSchG}$ eingefügte Abs. 6a bestimmt, daß in vereinfachten Verfahren über den Antrag bei Neugenehmigungen (nach $\S 4 \mathrm{BImSchG)} \mathrm{innerhalb} \mathrm{einer} \mathrm{Frist} \mathrm{von}$ drei Monaten, bei Änderungsgenehmigungen (nach § 15a BImSchG) innerhalb einer Frist von sechs Monaten (\$ 15a Abs. 1 Satz 2 BImSchG) zu entscheiden ist. Dabei ist eine Fristverlängerung um jeweils weitere drei Monate zulässig, wenn »dies wegen der Schwierigkeit der Prüfung oder aus Gründen, die dem Antragsteller zuzurechnen sind, erforderlich ist.« Für diesen Fall ist der Genehmigungsbehörde eine Begründungspflicht gegenüber dem Antragsteller auferlegt.

Es fällt schwer, plausible Gründe für die Festsetzung einer doppelt so langen Regelfrist für Änderungs- gegenüber Neugenehmigungen auszumachen. Es spricht nichts dafür anzunehmen, daß die Änderung einer bestehenden Anlage grundsätzlich mehr und/oder größere Probleme bei ihrer immissionsschutzrechtlichen Prüfung aufwirft, als dies zuvor bei ihrer (Erst-) Errichtung der Fall gewesen sein dürfte. ${ }^{300}$ Im übrigen enthalten die einschlägigen gesetzlichen Bestimmungen auch keinerlei Anhaltspunkte

298 Zur Dominanz des ökonomischen Arguments der Standortsicherung in der Umweltschutzgesetzgebung der 90er Jahre vgl. Caspar 1995 und Koch 1997, S. 53ff. m.w.N. (wortgleich mit Ders. 1996, S. 40ff.)

299 Die entsprechenden Änderungen erfolgten in Verbindung mit dem Investitionserleichterungs- und Wohnbaulandgesetz vom 22. April 1993.

300 Zweifel an einer sachlichen Grundlage nähren auch Ungereimtheiten der gesetzlichen Bestimmungen selbst. Während bei Neugenehmigungen für vereinfachte und (grundsätzlich mit Öffentlichkeitsbeteiligung einhergehende) »normale« Verfahren unterschiedliche Regelfristen von drei bzw. sieben Monaten gelten ( $\S 10$ Abs. 6a BImSchG), unterliegen Änderungsgenehmigungen beider Verfahrensarten der einheitlichen Frist von sechs Monaten. Das bedeutet, daß auf der einen Seite Änderungsgenehmigungen in vereinfachten Verfahren (ohne Öffentlichkeitsbeteiligung) 
dafür, daß sich die Unterscheidung von Neu- und Änderungsgenehmigung einem anderen Gesichtspunkt als dem rein rechtsimmanenten des Bestandsschutzes, den Anlagenänderungen, nicht aber Neuerrichtungen genießen, im wesentlichen verdankt. ${ }^{301}$

Ebenfalls mit dem Ziel der Verfahrensbeschleunigung wurde $\S 11$ der 9. BImSchV neu gefaßt, ${ }^{302}$ der die Beteiligung der in ihren Belangen betroffenen Fachbehörden regelt. Ihnen soll die Genehmigungsbehörde für die Erarbeitung ihrer Stellungnahme eine Frist von einem Monat setzen.

Den genannten Fristvorgaben kommt ausschließlich eine verwaltungsinterne Bindungskraft zu, nicht aber auch eine gegenüber dem Antragsteller. Das bedeutet insbesondere, daß bloßer Fristablauf keinesfalls die Genehmigung ersetzt. ${ }^{303}$ Entsprechend sind die Folgen von Fristüberschreitung auf den innerbehördlichen Bereich begrenzt. Da zudem die Regelfristen nicht mit Sanktionen im Falle ihrer Überschreitung verbunden sind, dürfte ihr präventives Potential insgesamt eher als gering einzuschätzen sein. ${ }^{304}$

Neben der Einführung von Regelfristen, mit der einer gemutmaßten Säumigkeit der öffentlichen Verwaltung gegengesteuert werden sollte, wurden mit der angesprochenen Novelle weitere, auf Verfahrensbeschleunigung zielende Änderungen im BImSchG und in der 9. BImSchV vorgenommen. Sie betreffen teils organisatorische Vorkehrungen wie etwa die umfassende Beratung des Vorhabenträgers bereits vor Antragstellung, ggf. auch unter Beiziehung anderer Behörden ( $\$ 2$ Abs. 2 Satz 2 der 9. BImSchV), oder die zeitgleiche (»sternförmige «) Übersendung der Antragsunterlagen an die Fachbehörden zur Stellungnahme ( $\$ 11$ Satz 2 der 9. BImSchV) und teils für den Antragsteller bedeutsamer - Erleichterungen bei den materiellen Genehmigungsvoraussetzungen wie etwa die Erweiterung der Zulassung

eine doppelt so lange Frist wie Neugenehmigungen eingeräumt wird, auf der anderen Seite für Änderungsgenehmigungen in Regelverfahren (mit Öffentlichkeitsbeteiligung) die Frist im Vergleich zu der für Neugenehmigungen eingeräumten dagegen sogar noch um einen Monat verkürzt ist. (Daß nach § 15a Abs. 2 BImSchG bei Änderungsgenehmigungen von der Öffentlichkeitsbeteiligung auf Antrag des Betreibers abgesehen werden kann, ist unabhängig von den Bestimmungen zur Regelfrist zu sehen). Insofern dürfte Hofmann 1994, S. 130, zuzustimmen sein, der das sachlich wenig überzeugende Fristenreglement mangelnder gesetzestechnischer Sorgfalt zuschreibt unter Verweis auf den großen Zeitdruck, unter dem die Novellierung erfolgt sei. In dieselbe Richtung geht auch die Kritik von Wulfhorst 1997, S. 165f. Die inzwischen in Kraft getretene 5. Novelle zum BImSchG vom Oktober 1996 hat die Inkonsistenz behoben und für vereinfachte Neu- und Änderungsgenehmigungsverfahren eine einheitliche Regelfrist von drei Monaten festgesetzt ( $\$ 16$ Abs. 3 BImSchG n.F.).

301 Vgl. zu den in der Genehmigungspraxis vielfach ähnlichen materiellen Problemlagen von Neu- und Änderungsgenehmigungsverfahren auch oben Kap. II, Ziff. 4.2.4.

302 Änderung der 9. BImSchV durch die Verordnung vom 20. April 1993 (BGB1. I S. 494).

303 Hansmann 1994, S. 58, Fn. 49. 
des vorzeitigen Beginns auch auf den Probebetrieb ( $§$ 15a Abs. 1 Nr. 2 BImSchG).

Die trotz der erfolgten Novelle anhaltende Kritik an der investitionshemmenden Ausgestaltung der Genehmigungsverfahren ${ }^{305}$ veranlaßte den Gesetzgeber, eine Unabhängige Expertenkommission zur Eruierung weiteren Beschleunigungspotentials einzusetzen. ${ }^{306}$ Die von der Kommission erarbeiteten Lösungsvorschläge ${ }^{307}$ konzentrieren sich vor allem auf eine Beschneidung der materiellen Anforderungen an die Genehmigungserteilung einschließlich des behördlichen Prüfumfangs zugunsten einer dem Anlagenbetreiber in höherem Maße überantworteten Selbstprüfung und Selbstüberwachung. ${ }^{308}$ Negative Auswirkungen auf den gesetzlich postulierten Schutz der Umwelt und der Allgemeinheit bzw. den Schutz Dritter werden explizit ausgeschlossen. ${ }^{309}$

Wenn die Kommission die angestrebte Verfahrensbeschleunigung in erster Linie durch Aufgabenentlastung der Behörden zu erreichen sucht, dagegen Überlegungen zur Änderung der Binnen- wie Außenorganisation der Verwaltung eher nur am Rande und allgemein gehalten anstellt (und sich im übrigen vom »Steuerungsinstrument « der Regelfristen nur wenig verspricht), so entbehrt dies nicht einer gewissen Konsequenz. Für die bisherige Genehmigungspraxis teilt sie ausdrücklich die Einschätzung, daß sich

304 Im Falle der Fristüberschreitung der Antragsbearbeitung durch die Genehmigungsbehörde kämen Disziplinarmaßnahmen des unmittelbaren Dienstvorgesetzten des jeweiligen Sachbearbeiters oder auch der vorgesetzten Behörde in Frage (was wiederum voraussetzt, daß diese überhaupt von dem Vorgang Kenntnis erlangt hat etwa im Wege einer Dienstaufsichtsbeschwerde des Antragstellers). Im Falle der Fristsäumnis der Fachbehörde in der Abgabe ihrer Stellungnahme kann die Genehmigungsbehörde gem. $\S 11 \mathrm{~S} .3$ der 9 . BImSchV davon ausgehen, daß diese sich nicht äußern will. Die (möglicherweise als Sanktion gegenüber der Fachbehörde gedachte) Regelung ist freilich kaum geeignet, die Position der Genehmigungsbehörde zu stärken. Diese kann nämlich beim Ausbleiben der angeforderten Stellungnahme nicht davon ausgehen, daß die fraglichen fachlichen Belange durch das anstehende Vorhaben auch tatsächlich nicht berührt sind. Dies hat zur Konsequenz, daß sie ggf. eigene Ermittlungen anstellen und/oder sich die notwendige Fachkenntnis durch Beauftragung eines Sachverständigen beschaffen muß (vgl. auch schon oben Ziff. 2.3).

305 Vgl. hierzu die (aus der Vielzahl einschlägiger Äußerungen ausgewählten) Nachweise bei Lübbe-Wolff 1995, S. 57, Fn. 2.

306 Nach ihrem Vorsitzenden »Schlichter-Kommission« genannt.

307 Vgl. hierzu statt vieler den Überblick des Kommissionsvorsitzenden: Schlichter 1995.

308 Ein Teil der Vorschläge ist inzwischen in die jüngste, 5. Novelle zum BImSchG vom Oktober 1996 eingegangen, so z.B. die (zunächst) bloße Anzeigepflicht auch »wesentlicher« Anlagenänderungen statt der bisherigen grundsätzlichen Genehmigungspflicht.

$309 \mathrm{Da}$ Anlaß besteht, diese Selbsteinschätzung von Grund auf in Zweifel zu ziehen, zeigt die ausführliche Kritik von Lübbe-Wolff 1995 an den Kommissionsvorschlägen. Vgl. auch Caspar 1995, S. $412 \mathrm{f}$. 
die deutschen Genehmigungsverfahren im internationalen Vergleich durch besondere Sorgfalt und Objektivität auszeichneten, dafür allerdings auch entsprechende Zeit beanspruchten. M.a.W. es wird eingeräumt, daß die Prüfgüte einer Genehmigung nicht unabhängig von dem eingesetzten Zeitaufwand ist. Des weiteren konzediert die Kommission im Hinblick auf die Dauer, daß die Behauptung überlanger Genehmigungszeiten in Deutschland gegenüber vergleichbaren anderen (westlichen) Industrieländern bislang eindeutiger empirischer Belege ermangele. ${ }^{310}$ Einschränkend geht sie allerdings davon aus, daß in Deutschland zum einen Verfahren mit »ungebührlich« langer Dauer (»Ausreißer«) einen nennenswerten Anteil ausmachten und zum anderen die Arbeitsweise der Behörden verbreitet als »nicht gerade zügig« gelte.

Die nachfolgenden Ergebnisse zur Verfahrensdauer verstehen sich ausdrücklich nicht als Beitrag zur Klärung der die Beschleunigungsdebatte in Gang haltenden Frage »überlanger « Genehmigungszeiten, und zwar vor allem aus zwei Gründen. Der eine und eher formale Grund ist der, daß sich die vorliegende Untersuchung vorrangig auf andere Aspekte des vereinfachten Genehmigungsverfahrens richtet. Der andere, gewichtigere Grund ist inhaltlicher Natur. Er betrifft die theoretisch-methodologischen Voraussetzungen, die erfüllt sein müßten, aber nicht erfüllt sind, um Bewertungen von Verfahrensdauern wie die einer »Überlänge « empirisch absichern zu können. Ohne den Maßstab einer wie immer definierten »angemessenen « und ggf. entsprechend anzustrebenden Genehmigungszeit, wird das Zeitkriterium zu einer absoluten Größe, deren theoretisches Optimum dann bei Null liegt. Inhaltlich liefe dies auf die Abschaffung des Genehmigungsverfahrens und damit jeglichen Genehmigungserfordernisses hinaus. D.h. selbst die kürzeste Verfahrensdauer muß letztlich als noch zu lang gelten. ${ }^{311}$ Eine »angemessene « Genehmigungszeit wird sich nicht definieren lassen, ohne (inhaltliche) Mindestanforderungen an den Verfahrensablauf wie etwa die Wahrnehmung der Rechte Dritter und an das Verfahrensergebnis wie etwa die Qualität der erteilten Genehmigung im Hinblick auf Prüftiefe und Einhaltung der rechtlich vorgegebenen immissionsschutzrechtlichen Standards einzubeziehen. Zwar ist zuzugeben, daß die Erfassung solcher qualitativer Faktoren ihrerseits Meßprobleme aufwirft. Das rechtfertigt aber nicht, die Verfahrensdauer zum praktisch alleinigen obersten Bewertungs-

310 Schlichter 1995, S. 174. Vgl. auch den Hinweis von Bohne 1994, S. 195, es fehlten »systematische, empirische Analysen der Dauer von Zulassungsverfahren und der verfahrensbestimmenden Faktoren für West- und Ostdeutschland, die sozialwissenschaftlichen Methodenanforderungen« genügten. In der seit Jahren laufenden Beschleunigungsdiskussion dominierten »Praktikeraussagen «, die »nicht immer frei « von Interessen seien und sich zudem an »fehlgeschlagenen« Einzelfällen orientierten.

$311 \mathrm{Vgl}$. auch die plastische Einkleidung des Problems in die Frage »wie schnell ist schnell genug?« bei Bohne 1994, S. 195. 
kriterium der Genehmigungspraxis zu erheben. Dem kommt indes der zweifelhafte »Vorzug « zu - wie die jüngsten Reformvorschläge, etwa die der $\gg$ Schlichter-Kommission «, belegen -, mit dem vermeintlich neutralen Argument der Zeitersparnis das primär angestrebte Ziel einer Absenkung der materiellen Genehmigungsstandards zu überdecken. ${ }^{312}$

Im Rahmen der vorliegenden Untersuchung erfolgt die Analyse der Verfahrensdauer ausschließlich in vergleichender Perspektive der beiden Genehmigungsmodelle. Sie nimmt dabei auch die verbreitete und auf den ersten Blick plausible Auffassung auf, daß die (baden-württembergische) Trennung von Genehmigungs- und technischer Fachbehörde im Vergleich zur (niedersächsischen) Integration der beiden Kompetenzen auf Grund des erhöhten Koordinationsaufwands eine längere Verfahrensdauer nach sich zieht. ${ }^{313}$ Zugunsten einer Verfahrensbeschleunigung gibt nicht nur die »Schlichter-Kommission « zu erwägen, verwaltungsrechtliche und fachtechnische Kompetenzen in einer Behörde zu vereinen. ${ }^{314}$ Als Bündelungsinstanz kommen grundsätzlich sowohl die Kommunalverwaltung als auch die staatliche Sonderverwaltung in Frage. ${ }^{315}$ Die aktuelle Entwicklung läuft allerdings auf eine Präferenz für die kommunale Lösung hinaus. ${ }^{316}$ Vor diesem Hintergrund hat das baden-württembergische Trennungsmodell wenig Chancen, zum Regelfall zu werden. Ob sich das niedersächsische Integra-

312 Ihre Kritik an den Reformvorschlägen der Schlichter-Kommission faßt Lübbe-Wolff 1995, S. 62, in dem Fazit zusammen, daß diese im Kern »dem in der deutschen Umweltpolitik derzeit dominierenden Trend [entsprechen], unter Lippenbekenntnissen zum Umweltschutz dessen Abbau zu betreiben.«

313 Vgl. etwa Dose 1997, S. 235 u. S. 419, Lübbe-Wolff 1996, S. 62f., Lorenz 1991, S. 256. Nach Lübbe-Wolff 1996, S. 62, verkörpert das Trennungsmodell eine »irrationale(.)«, insbesondere »extrem zeitfressend(e)« Organisationsstruktur.

314 Schlichter 1995, S. 179.

315 Vgl. Lübbe-Wolff 1990, S. 29f. Die »Schlichter-Kommission« (wie vorhergehende Anm.) beispielsweise plädiert dagegen für eine Bündelung bei der allgemeinen Verwaltung.

316 Dafür spricht, daß in verschiedenen Bundesländern im Rahmen der Reformbestrebungen zur »Verschlankung« der öffentlichen Verwaltung staatliche Sonderbehörden aufgelöst und deren Aufgaben den Kommunen zugewiesen wurden, beispielsweise in Baden-Württemberg und in Niedersachsen (oder auch in Nordrhein-Westfalen) die Ämter für Wasser- und Bodenwirtschaft bzw. für Wasserwirtschaft. Standen bislang die staatlichen Gewerbeaufsichtsämter nicht auf der Liste potentiell aufzuhebender Sonderbehörden, so scheint es neuerdings zumindest in NordrheinWestfalen Überlegungen zu geben, auch diese Ämter (nach erst jüngst erfolgter Integration der Wasser- und Abfallwirtschaft in staatliche Umweltämter umbenannt bei gleichzeitiger Auslagerung des Arbeitsschutzes in eigene staatliche Ämter) nicht von vornherein grundsätzlich auszunehmen. 
tionsmodell auf der Ebene der Sonderverwaltung gegen das kommunale Konkurrenzmodell ${ }^{317}$ behaupten kann, bleibt abzuwarten.

Dem nachfolgenden Vergleich werden die im Erhebungszeitraum geltenden, 1993 eingeführten Regelfristen von drei Monaten für Neu- und sechs Monaten für Änderungsgenehmigungen zugrunde gelegt. Zusätzlich werden die Verfahrensdauern auch auf die inzwischen geltende einheitliche Dreimonatsfrist bezogen. ${ }^{318}$

\subsection{Variationen der Gesamtdauer}

\subsubsection{Mögliche Bezugspunkte der Berechnung}

Als rein quantitatives (intervallskaliertes) Merkmal erscheint die Verfahrensdauer auf den ersten Blick als »objektiv « leicht bestimmbare und von daher unproblematische Größe. Die inhaltliche Aussagekraft der gemessenen Dauer hängt freilich davon ab, wie jeweils Anfangs- und Endpunkt definiert bzw. datiert werden.

Wenig Variationsmöglichkeiten bestehen bei der Festlegung des Endpunkts. Das Verfahren erreicht offenkundig mit dem Erlaß des Genehmigungsbescheids und dessen Kenntnisgabe an den Antragsteller seinen formellen Abschluß. Allerdings gibt es durchaus Gründe, die dafür sprechen, bei Widersprüchen des Antragstellers gegen den Genehmigungsbescheid erst den Zeitpunkt der Entscheidung in Form der Abhilfeprïfung durch die Genehmigungsbehörde oder sogar erst den Zeitpunkt der Entscheidung durch Widerspruchsbescheid der (übergeordneten) Widerspruchsbehörde im Falle des (bei negativem Ausgang der Abhilfeprüfung) aufrechterhaltenen Widerspruchs als definitive Beendigung des (verwaltungsförmigen) Genehmigungsverfahrens zu betrachten. ${ }^{319}$ Schließlich ist der Widerspruch immer (auch) Folge des Verfahrens selbst insofern, als es den Behörden

$317 \mathrm{Zu}$ der mit dieser Lösung verbundenen Gefahr einer stärkeren Einflußnahme kommunalpolitischer Interessen auf die Vollzugspraxis vgl. oben Anm. 43. Möglicherweise wäre ein solcher Effekt aus der Sicht derer, die die »Investitionshemmnisse« des geltenden Genehmigungsrechts beklagen, nicht unbedingt unerwünscht.

318 Den Grund dafür liefert nicht zuletzt die Beobachtung, daß sich die Sachbearbeiter der untersuchten Verfahren selbst nicht an den gestatteten längeren Regelfristen für Änderungsgenehmigungen orientierten, sondern bei diesen ebenfalls von einer Dreimonatsfrist ausgingen. Dies deutet darauf hin, daß die Praxis sachlich kaum zu rechtfertigende Differenzierungen zu ignorieren geneigt ist, auch wenn sie solche Differenzierungen zu ihren Gunsten nutzen könnte.

319 Ein gegen den Bescheid der Widerspruchsbehörde eingeleitetes verwaltungsgerichtliches Verfahren sollte dagegen nicht mehr dem - letztlich zwar auch hier als Auslöser zu betrachtenden - Genehmigungsverfahren zugerechnet werden, da nunmehr die Sphäre der Verwaltung verlassen ist. 
ausweislich der Gegenwehr des Antragstellers im Ergebnis nicht gelungen ist, die anstehenden Probleme zureichend zu erörtern oder in einer diesen überzeugenden Weise zu lösen. ${ }^{320}$ Die durch ein »schnelles « Verfahren erzielte Zeitersparnis kann durch die nachträgliche Widerspruchsbearbeitung aufgewogen, wenn nicht gar am Ende durch den zusätzlichen Zeitverbrauch »überkompensiert« werden. In diesen Fällen erfaßt die Orientierung des Verfahrensabschlusses am Erlaß des Genehmigungsbescheids lediglich einen Teil des von der Genehmigungsbehörde am Ende für das Verfahren bis zur (verwaltungsförmigen) Rechtskraft der Genehmigungsentscheidung erbrachten Zeitaufwands. Je höher der Anteil an Verfahren mit Widerspruchseinlegung ausfällt, umso mehr wirkt es sich aus, ob die Zeitspanne der behördlichen Widerspruchsbearbeitung der Verfahrensdauer zugerechnet wird oder nicht. In der vorliegenden Untersuchung wird die Widerspruchsphase auf die Abhilfeprüfung der Genehmigungsbehörde aus den oben genannten Gründen (Ziff. 3.4.2 mit Anm. 289) begrenzt. $^{321}$

Weitreichendere Konsequenzen für die Aussagekraft von Genehmigungsdauern verbinden sich mit der Bestimmung des Verfahrensbeginns. Hier bieten sich grundsätzlich drei verschiedene Bezugspunkte an.

a) Ein erster Bezugspunkt orientiert sich am Beginn der gesetzlich vorgegebenen Regelfristen. Deren Laufzeit setzt ein mit Vollständigkeit der beizubringenden Unterlagen. ${ }^{322}$ Die Vollständigkeit der Unterlagen hat die Genehmigungsbehörde, wie oben erwähnt (Ziff. 2.1), nach Eingang »unverzüglich « zu prüfen und ggf. den Antragsteller zur Ergänzung innerhalb einer »angemessenen « Frist aufzufordern. ${ }^{323}$ Mit Feststellung der Vollständigkeit hat die Genehmigungsbehörde den Antragsteller »über den geplanten zeitlichen Ablauf des Genehmigungsverfahrens zu unterrichten. $\ll^{324}$ Die Anbindung der Fristlaufzeit an die Unterlagenvollständigkeit kommt insofern der Genehmigungsbehörde entgegen, als ihr Verzögerungen bei der dem Antragsteller obliegenden Unterlagenbeibringung nicht angelastet

320 Es handelt sich hier lediglich um eine (empirische) ex post-Feststellung und nicht um eine Zurechnung von Verantwortung für das »Scheitern«. Mit ihrer oben (Ziff. 3.4.2) aufgezeigten hohen Abhilfebereitschaft gegenüber Widersprüchen geben die Genehmigungsbehörden bzw. die betroffenen Fachbehörden im Ergebnis zu erkennen, die ihnen verfügbaren Handlungsspielräume im Verfahren selbst nicht situationsgerecht genutzt zu haben. Darunter fallen auch möglicherweise unternommene Versuche, »striktere « Genehmigungsbedingungen durchzusetzen als von den rein rechtlichen Entscheidungsgrundlagen her (im Streitfalle) legitimierbar.

321 Zur Dauer dieser Phase s. unten Ziff. 4.3.5.

322 Das ergibt sich aus der Formulierung von $\S 10$ Abs. 6a BImSchG: »Über den Genehmigungsantrag ist nach Eingang des Antrags und der nach Absatz 1 Satz 2 einzureichenden Unterlagen innerhalb einer Frist von [...] zu entscheiden.« (Hervorheb. von $\mathrm{mir}-\mathrm{Br}$.)

$323 \S 7$ Abs. 1 der 9. BImSchV.

$324 \S 7$ Abs. 2 der 9. BImSchV. 
werden. Im Untersuchungszeitraum sah das Gesetz für die Durchführung der Vollständigkeitsprüfung keine Frist vor. Eine solche ist inzwischen aber eingeführt worden. ${ }^{325}$

Obwohl die genannten gesetzlichen Vorgaben grundsätzlich einen zureichend operationalen Verfahrensbeginn markieren, stößt dessen Anwendung auf die Genehmigungspraxis auf Schwierigkeiten. Diese resultieren hauptsächlich daraus, daß die Genehmigungsbehörde nur begrenzt über die erforderlichen materiellen Kompetenzen verfügt, die Vollständigkeit der Antragsunterlagen im Hinblick auf sämtliche betroffene fachliche Belange zu beurteilen. Von daher neigt sie eher dazu, sich diesbezüglich gegenüber dem Antragsteller nicht festzulegen und sich die Option späterer (weiterer) Nachforderungen vorzubehalten. Nur in einer Minderzahl der untersuchten Verfahren wurde deren offizielle Einleitung in beiden Genehmigungsmodellen - wenn auch mit graduellen Unterschieden - ausdrücklich von der Komplettierung der vorgelegten Unterlagen abhängig gemacht (vgl. oben Ziff. 2.2). Entsprechend konnte die Mehrheit der Antragsteller davon ausgehen, daß das Verfahren mit dem Eingang ihres Antrags begonnen hat. Im Ergebnis führt die Präferenz der Genehmigungsbehörde, die Frage der Unterlagenvollständigkeit - von eindeutigen Fällen abgesehen - offenzuhalten, dazu, daß sie das gesetzlich eingeräumte Entlastungspotential bei der Kalkulation der Regelfrist nicht ausschöpft. ${ }^{326}$

b) Als zweiter Bezugspunkt für den Verfahrensbeginn kommt der Antragseingang in Betracht. Spätestens ab diesem Zeitpunkt ist die Genehmigungsbehörde mit dem Genehmigungsbegehren de facto befaßt, und zwar in Form der erforderlichen Vollständigkeitsprüfung. Da eine solche Kontrolle, um sie seriös zu absolvieren, bereits ein »Einsteigen in die Sache« notwendig macht, kann sie u. U. einen beträchtlichen Arbeits- und Zeitaufwand mit sich bringen. Wie gerade dargelegt, ließen die Behörden gleichwohl die Mehrheit der Verfahren bei Antragseingang offiziell beginnen und leiteten die Fachbehördenbeteiligung ein unabhängig davon, ob noch Ergänzungen der Unterlagen erforderlich waren. ${ }^{327}$ Auf Grund dieser Praxis führt die Differenzierung zwischen Unterlagenvollständigkeit und Antrags-

$325 \S 7$ Abs. 1 Satz 1 u. 2 der 9. BImSchV n.F. Siehe auch näher unten Anm. 328 und Ziff. 4.3.2.

326 Unabhängig davon muß sich die gesetzliche Bestimmung fragen lassen, ob es realistisch ist zu erwarten, daß eine einzelne (Genehmigungs-) Behörde über die notwendige Kompetenz verfügt, die geforderte umfassende, wegen des Konzentrationsprinzips sich auf die unterschiedlichsten fachlichen Belange erstreckende Vollständigkeitsbeurteilung vor Beteiligung der jeweiligen Fachbehörden zu leisten.

327 Dies spiegelt sich auch in den Genehmigungsbescheiden wider, die nur zum kleineren Teil der Fälle darauf hinweisen, daß der ursprünglich eingereichte Antrag unvollständig war. Eine Datierung der Unterlagenvollständigkeit ist aber auch dann eher die Ausnahme. 
eingang als Bezugspunkt für den Verfahrensbeginn nur zu geringen Unterschieden in der Verfahrensdauer, wie nachfolgend gezeigt wird. ${ }^{328}$

c) Als dritter Bezugspunkt für den Verfahrensbeginn bietet sich der Zeitpunkt an, in dem die Genehmigungsbehörde erstmals mit dem Genehmigungsgegenstand konfrontiert ist und von dem ab sie Arbeit und Zeit in den prospektiven Genehmigungsvorgang investiert. Dies war bei den untersuchten Verfahren beider Modelle, wie dargelegt, ${ }^{329}$ in der großen Mehrheit der Fall. Die hier unter den Begriff der » Vorantragsberatung « gebündelten Aktivitäten der Genehmigungsbehörde galten dabei nicht nur einer Beratung i.S. einer verfahrensrechtlich und ggf. auch fachtechnischen Hilfestellung für die Erarbeitung der erforderlichen Antragsunterlagen. In nicht geringem $\mathrm{Maße}$ sah sie sich dem oft mühsamen Unterfangen ausgesetzt, $\mathrm{Be}$ treiber nicht genehmigter bzw. sanierungsbedürftiger Anlagen oder Anlagenteile zur (nachträglichen) Beantragung der gebotenen Genehmigung zu veranlassen. ${ }^{330}$ Von daher kann allein die Vorantragsberatung bis zum Antragseingang im Einzelfall erhebliche Zeit in Anspruch nehmen. Berücksichtigt man, daß die Genehmigungsbehörde (gem. § 2 Abs. 2 Satz 1 der 9. BImSchV) zur Beratung verpflichtet ist, sobald sie vom Vorhabensträger über seine Planung unterrichtet wird, erheben sich grundsätzliche Bedenken dagegen, dieses der förmlichen Antragstellung vorgelagerte Stadium des Genehmigungsverfahrens als lediglich »inoffizielles « zu betrachten und von der restlichen, »offiziellen « Laufzeit abzutrennen. Gegen eine solche Diskreditierung der Vorantragsphase spricht zudem, daß es der Genehmigungsbehörde (gem. Satz 2 der genannten Vorschrift) freisteht, ggf. zur Erfüllung ihrer Beratungspflicht bei der Antragserstellung auch schon andere Behörden hinzuzuziehen. Eine umfängliche und gründliche Erörterung der Genehmigungsvoraussetzungen des geplanten Vorhabens dürfte sich auch in zeitlicher Hinsicht auf die nach Antragseingang durchzuführenden Verfahrensschritte i.d.R. entlastend auswirken.

Im Unterschied zum Bezugspunkt des amtlichen »Erstkontakts« mit dem Genehmigungsgegenstand als Kriterium für den Verfahrensbeginn ist den anderen beiden Bezugspunkten gemeinsam, daß über deren zeitliche Fixierung die Genehmigungsbehörde in Richtung eines späteren oder aber auch

328 Allein von daher dürfte von der im Rahmen der 5. Novelle zum BImSchG vom Oktober 1996 für die Vollständigkeitsprüfung eingeführten Regelfrist von einem Monat nach Antragseingang mit einmaliger Verlängerungsmöglichkeit von 2 Wochen $(\S 7$ Abs. 1 Satz 1 u. 2 der 9. BImSchV n.F.) kein ins Gewicht fallender Beschleunigungseffekt zu erwarten sein. Unabhängig davon halten Kritiker der Neuregelung entgegen, daß diese zu zusätzlichen Belastungen der Verwaltung führe und damit einer zügigen Verfahrensdurchführung eher abträglich sei (vgl. die Kommentierung zu $§ 7$ von Czajka 1996, S. 3).

329 Vgl. oben Ziff. 1.3.1. mit Tab. 8.

$330 \mathrm{Vgl}$. oben Ziff. 1.2. und 1.3.4. 
eines früheren Termins - in Grenzen - verfügen kann. Für die Feststellung der Unterlagenvollständigkeit wurde bereits darauf hingewiesen, daß die Genehmigungsbehörden in dieser Frage eher Zurückhaltung walten ließen und damit in Kauf nahmen, legitime Spielräume, den Beginn der gesetzlichen Regelfrist hinauszuschieben, zu verschenken. Dies gewissermaßen kompensierend, bevorzugten sie es, ihre Bemühungen um Verkürzung der Regellaufzeit in die Vorantragsphase vorzuziehen und das Verfahren formell erst mit dem Eingang eines prüffähigen Antrags beginnen zu lassen. Theoretisch könnte die gesamte materielle Prüfarbeit, die den Kern eines Genehmigungsverfahrens ausmacht, in das inoffizielle Vorstadium ausgelagert werden. Dem anschließenden offiziellen Verfahren fiele dann nur noch die weniger zeitaufwendige Aufgabe der förmlichen Bestätigung des vorverhandelten Genehmigungsinhalts zu. Die Praxis gibt sich freilich, wie sich noch zeigen wird, weit weniger radikal als die Theorie.

Jeder der hier erörterten Bezugspunkte für den Verfahrensbeginn bzw. das Verfahrensende reflektiert eine andere Auffassung davon, welche Aktivitäten der Hauptbeteiligten schon bzw. noch Bestandteil des Verfahrens sind. Dabei hat sich herausgestellt, daß sowohl die Vollständigkeit der Antragsunterlagen als auch der Antragseingang in gewissen Grenzen zur Disposition der Genehmigungsbehörde stehen und von daher nicht als »objektiver« Verfahrensbeginn behandelt werden können. In Abhängigkeit von den beiden Bezugspunkten variiert die jeweilige diesen vorgelagerte, nicht zum Verfahren gerechnete Zeitspanne, in der die Genehmigungsbehörde faktisch bereits mit dem Genehmigungsgegenstand befaßt ist und Vorentscheidungen für die weitere Verfahrensentwicklung trifft. Insofern repräsentiert allein der Zeitpunkt des Erstkontakts der Genehmigungsbehörde mit dem Genehmigungsbegehren einen ihrer strategischen Verfügung entzogenen Verfahrensbeginn. Er ist auch der einzige, der den gesamten, von der Genehmigungsbehörde tatsächlich erbrachten Zeit- und Arbeitsaufwand eines Genehmigungsverfahrens erfaßt. ${ }^{331}$

331 Die Regelfristen in der gesetzlich definierten Form blenden einen Teil dieses Aufwands aus. Das hat nur auf den ersten Blick für die Behörde den Vorteil, daß sie das Risiko von Fristüberschreitungen durch Ausdehnung der »inoffiziellen « Phase minimieren kann. Auf der anderen Seite hält sie damit jene tatsächlich erbrachte Verwaltungsleistung unsichtbar mit der nachteiligen Folge, daß sie offiziell auch als entsprechend geringer belastet gilt. 


\subsubsection{Verschiedene Gesamtdauern im Vergleich}

\subsubsection{Dauer ab Vollständigkeit der Antragsunterlagen bis zum Erlaß des Genehmigungsbescheids}

Die kürzeste Gesamtdauer eines Genehmigungsverfahrens errechnet sich, wenn man den Zeitpunkt der Vollständigkeit der Antragsunterlagen als Verfahrensbeginn und den Erlaß des Genehmigungsbescheids als Verfahrensende zugrunde legt. Tab. 36.1 zeigt die Verteilung der untersuchten Verfahren auf verschiedene Zeitklassen der so definierten Gesamtdauern.

Tab. 36.1

Verfahrensdauer ab Vollständigkeit der Antragsunterlagen bis Erlaß Genehmigungsbescheid (Häufigkeitsverteilung)

\begin{tabular}{|l|c|c|c|}
\cline { 2 - 4 } & GAA-N & $L R A-1$ & $L R A-2$ \\
\hline \multirow{2}{*}{ Bis zu drei Monaten } & 15 & 11 & 8 \\
& $25,9 \%$ & $25,6 \%$ & $33,3 \%$ \\
\hline \multirow{2}{*}{ Über drei bis sechs Monate } & 24 & 17 & 9 \\
& $41,4 \%$ & $39,5 \%$ & $37,5 \%$ \\
\hline Über sechs bis zwölf Monate & 9 & 8 & 3 \\
\hline \multirow{2}{*}{ Über zwölf Monate* } & $15,5 \%$ & $18,6 \%$ & $12,5 \%$ \\
\hline \hline \multirow{2}{*}{ Verfahren gesamt } & 10 & 7 & 4 \\
\hline
\end{tabular}

* Maximaler Wert beim GAA-N: 33,5 Mon., LRA-1: 50,4 Mon., LRA-2: 20,1 Mon.

Die Befunde zeigen zum einen, daß auf die einzelnen Häufigkeitsklassen beim GAA-N und beim LRA-1 nahezu gleich große Anteile entfallen. Die beiden Genehmigungsmodelle unterscheiden sich also in dieser Hinsicht kaum voneinander. ${ }^{332} \mathrm{Zum}$ anderen stellt sich heraus, daß bei beiden Behörden lediglich ein Viertel der Verfahren $(25,9 \%$ bzw. $25,6 \%)$ innerhalb der Regelfrist von drei Monaten und weitere rd. 40\% (41,4\% bzw. 39,5\%) innerhalb einer Frist von sechs Monaten erledigt wurden. Entsprechend be-

332 Die Anteile beim LRA-2 weisen lediglich für die erste Klasse (»Bis zu drei Monaten «) einen etwas höheren Anteil aus. Diese Abweichung von den entsprechenden Werten der beiden anderen $\ddot{m}$ ter ist aber nicht signifikant. 
nötigte das verbleibende Drittel (32,8\% bzw. 34,9\%) länger als sechs Monate, die Hälfte davon sogar länger als 12 Monate.

Vor allem die erheblichen Kontingente an Fällen mit »Überlänge« sorgen dafür, daß die durchschnittliche Verfahrensdauer mit 6,0 Monaten beim GAA-N und 7,3 Monaten beim LRA-1 doppelt so hoch (bzw. beim LRA-1 noch etwas darüber) ${ }^{333}$ ausfällt, wie es die dreimonatige Regelfrist für den $»$ Normalfall« vorsieht $(\mathrm{Tab} .36 .11)$.

Tab. 36.11

Verfahrensdauer ab Vollständigkeit der Antragsunterlagen bis Erlaß Genehmigungsbescheid (statistische Parameter in Monaten)*

\begin{tabular}{|l|c|c|c|}
\cline { 2 - 4 } \multicolumn{1}{c|}{} & GAA-N & LRA-1 & LRA-2 \\
\hline Arith. Mittel & 6,02 & 7,27 & 5,43 \\
\hline Varianz & 21,63 & 47,17 & 16,58 \\
\hline Median & 4,20 & 4,66 & 4,49 \\
\hline Minimum & 0,82 & 1,41 & 0,26 \\
\hline Maximum & 19,64 & 31,71 & 15,31 \\
\hline \hline Gesamt & 57 & 42 & 23 \\
\hline
\end{tabular}

* Ohne Extremwerte.

Dabei werden die Durchschnittswerte durch Fälle mit besonders langer Dauer belastet, was sich auch in den deutlich niedrigeren Medianwerten (4,2 bzw. 4,7 Mon.), unter bzw. über dem jeweils die Hälfte der Verfahren liegt, niederschlägt. Speziell der höhere Durchschnittswert beim LRA-1 resultiert in erster Linie daraus, daß sich hier einige Verfahren über $20 \mathrm{Mo-}$ nate und länger hingezogen haben, was bei der niedersächsischen Behörde nicht vorgekommen ist. ${ }^{334}$ Dies drückt sich auch in der deutlich höheren Va-

333 Die Differenz der Mittelwerte ist allerdings nicht signifikant $\left(\mathrm{t}=1,079<\mathrm{t}_{(0,90 ; 97)}=\right.$ 1,290. Das gilt auch für die Differenz der Varianzen unter der Bedingung eines hohen Sicherheitsniveaus von $p=0,001$ für die Gleichheitshypothese $\left(F=2,18<\mathrm{F}_{(0,999}\right.$; $41,56)=2,31$ ).

334 Bei diesen Dauern handelt es sich nicht um Extremwerte der Rohdatenverteilung. Solche Extremwerte blieben (hier wie auch im weiteren) bei der Berechnung der statistischen Parameter grundsätzlich unberücksichtigt. 
rianz (47,2 gegenüber 21,6 Mon.) und dem höheren Maximalwert (31,7 gegenüber 19,6 Mon.) aus. ${ }^{335}$

Das Bild ändert sich nicht grundlegend, wenn man die Verfahren mit Blick auf die im Untersuchungszeitraum geltenden unterschiedlichen Regelfristen von drei und sechs Monaten für Neu- bzw. Änderungsgenehmigungen entsprechend differenziert (Tab. 36.2).

Tab. 36.2

Verfahrensdauer ab Vollständigkeit der Antragsunterlagen bis Erlaß Genehmigungsbescheid, getrennt nach Neu- und Änderungsgenehmigungen (Häufigkeitsverteilung)*

\begin{tabular}{|l|c|c|c|c|c|c|}
\cline { 2 - 7 } \multicolumn{1}{c|}{} & \multicolumn{2}{c|}{$G A A-N$} & \multicolumn{2}{c|}{$L R A-1$} & \multicolumn{2}{c|}{ LRA-2 } \\
\cline { 2 - 7 } & Änd.-G & Neu-G & Änd.-G & Neu-G & Änd.-G & Neu-G \\
\hline \multirow{2}{*}{ Bis zu drei Monaten } & $\mathbf{1 2}$ & 3 & $\mathbf{5}$ & 6 & $\mathbf{4}$ & 4 \\
& $\mathbf{2 8 , 6 \%}$ & $18,8 \%$ & $\mathbf{1 9 , 2 \%}$ & $35,3 \%$ & $\mathbf{3 3 , 3 \%}$ & $33,3 \%$ \\
\hline \multirow{2}{*}{ Über drei bis sechs Monate } & $\mathbf{1 7}$ & 7 & $\mathbf{1 2}$ & 5 & $\mathbf{3}$ & 6 \\
\hline \multirow{2}{*}{ Über sechs bis zwölf Monate } & $\mathbf{4 0 , 5} \%$ & $43,8 \%$ & $\mathbf{4 6 , 2} \%$ & $29,4 \%$ & $\mathbf{2 5 , 0 \%}$ & $50,0 \%$ \\
\hline \multirow{2}{*}{ Über zwölf Monate } & $\mathbf{1 4 , 3 \%}$ & $18,8 \%$ & $\mathbf{1 1 , 5 \%}$ & $29,4 \%$ & $\mathbf{8 , 3 \%}$ & $16,7 \%$ \\
\hline \hline \multirow{2}{*}{ Verfahren gesamt** } & $\mathbf{7}$ & 3 & $\mathbf{6}$ & 1 & $\mathbf{4}$ & 0 \\
& $\mathbf{1 6 , 7 \%}$ & $18,8 \%$ & $\mathbf{2 3 , 1 \%}$ & $5,9 \%$ & $\mathbf{3 3 , 3 \%}$ & $0,0 \%$ \\
\hline
\end{tabular}

* Angesichts der teilweise niedrigen absoluten Grundgesamtheiten dient die Prozentuierung lediglich der näherungsweisen (heuristischen) Vergleichbarkeit der auf die einzelnen Kategorien entfallenden absoluten Häufigkeiten.

** Abweichungen von $100,0 \%$ durch Rundungen bei den prozentualen Einzelhäufigkeiten.

Danach konnten jeweils rd. zwei Drittel der Änderungsgenehmigungsverfahren beim GAA-N $(69,0 \%)$ und beim LRA-1 $(65,4 \%)$ die Sechsmonatsfrist einhalten. Die für diese Verfahren mit der jüngsten Novelle aus 1996 geltende Regelfrist von nunmehr ebenfalls drei Monaten erfüllen allerdings nicht einmal ein Drittel von ihnen, wobei der Anteil beim GAA-N mit $28,6 \%$ deutlich, aber nicht statistisch signifikant über dem beim LRA-1 mit

335 Die Differenz der Varianzen ist unter einem Sicherheitsniveau für die Gleichheitshypothese von $\mathrm{p}=0,001$ nicht signifikant (vgl. vorletzte Anm.), unter dem niedrigeren Sicherheitsniveau von $\mathrm{p}=0,005$ aber signifikant $\left(\mathrm{F}=2,18>\mathrm{F}_{(0,999 ; 41,56)}=\right.$ $2,08)$. 
$19,2 \%$ liegt. Schneidet somit hier die niedersächsische Genehmigungsbehörde etwas günstiger ab als die baden-württembergische, verhält es sich bei den Neugenehmigungsverfahren mit Anteilen von 18,8\% beim GAA-N bzw. 35,3\% beim LRA-1 umgekehrt. ${ }^{336}$ In beiden Modellen überschreitet das Gros der Verfahren den gesetzlich zugebilligten Zeitrahmen, überdies in rd. einem Drittel der Neu- wie auch der Änderungsgenehmigungsfälle mit Dauern von mehr als sechs bzw. mehr als 12 Monaten in einem erheblichen Maße. ${ }^{337}$

Tab. 36.21

Verfahrensdauer ab Vollständigkeit der Antragsunterlagen bis Erlaß Genehmigungsbescheid, getrennt nach Neu- und Änderungsgenehmigungen (statistische Parameter in Monaten) ${ }^{*}$

\begin{tabular}{|l|c|c|c|c|c|c|}
\cline { 2 - 8 } \multicolumn{1}{c|}{} & \multicolumn{2}{c|}{$G A A-N$} & \multicolumn{2}{c|}{$L R A-1$} & \multicolumn{2}{c|}{ LRA-2 } \\
\cline { 2 - 8 } & Änd.-G & Neu-G & Änd.-G & Neu-G & Änd.-G & Neu-G \\
\hline Arith. Mittel & $\mathbf{5 , 9 7}$ & 6,15 & $\mathbf{8 , 2 7}$ & 5,81 & $\mathbf{6 , 5 5}$ & 4,41 \\
\hline Varianz & $\mathbf{2 3 , 2 1}$ & 18,53 & $\mathbf{5 9 , 4 2}$ & 27,90 & $\mathbf{2 7 , 6 6}$ & 5,62 \\
\hline Median & $\mathbf{4 , 1 6}$ & 5,12 & $\mathbf{4 , 9 8}$ & 3,71 & $\mathbf{4 , 4 9}$ & 4,53 \\
\hline Minimum & $\mathbf{0 , 8 2}$ & 1,31 & $\mathbf{2 , 1 0}$ & 1,41 & $\mathbf{1 , 9 7}$ & 0,26 \\
\hline Maximum & $\mathbf{1 9 , 6 4}$ & 16,59 & $\mathbf{3 1 , 7 1}$ & 22,95 & $\mathbf{1 5 , 3 1}$ & 9,61 \\
\hline \hline Gesamt & $\mathbf{4 2}$ & 15 & $\mathbf{2 5}$ & 17 & $\mathbf{1 1}$ & 12 \\
\hline
\end{tabular}

* Ohne Extremwerte.

Die Betrachtung der durchschnittlichen Verfahrensdauer (Tab. 36.21) zeigt für das GAA-N keine größere Differenz zwischen Neu- und Änderungsgenehmigungen, die mit 6,0 $(5,97)$ bzw. 6,2 Monaten beide in Höhe der Sechsmonatsfrist liegen. Das gilt auch für die Dauer der Neugenehmigungen beim LRA-1 mit einem Durchschnitt von 5,8 Monaten. Dagegen kommen die dortigen Änderungsgenehmigungen auf einen um mehr als zwei

336 Aber auch diese Differenz bleibt - angesichts der absolut geringen Anzahl von Neugenehmigungen - unter der Signifikanzgrenze $\left(\mathrm{chi}^{2}=1,23<2,71\right.$, für $\mathrm{p}=0,1$ und df $=1$.).

337 Siehe im einzelnen die in Tab. 36.2 in den entsprechenden Kategorien ausgewiesenen Anteile. 
Monate darüber liegenden (signifikant höheren) Wert von 8,3 Monaten. ${ }^{338}$ Dies bedeutet, daß »überlange« Dauern hauptsächlich Änderungsgenehmigungs- und weniger Neugenehmigungsverfahren betrafen, was sich auch an dem mit 5,0 (4,98) Monaten erheblich unter dem Mittelwert liegenden Median ablesen läßt.

\subsubsection{Dauer ab Antragseingang bis zum Erlaß des Genehmigungsbescheids}

Eine zwangsläufig längere Gesamtdauer ergibt sich, wenn man den der Feststellung der Unterlagenvollständigkeit vorgelagerten Zeitpunkt des Antragseingangs bei der Genehmigungsbehörde als Verfahrensbeginn zugrunde legt.

Tab. 37.1 zeigt zunächst die Häufigkeitsverteilung der Verfahren auf verschiedene Zeitklassen. Wie man ihr entnehmen kann, sind die Anteile in den einzelnen Kategorien nicht entscheidend gegenüber denen in Tab. 36.1 (Ziff. 4.2.2.1) verschoben. Die beiden unteren, eher kürzere Dauern repräsentierenden Klassen sind gleich oder etwas geringer und die beiden oberen, eher längere Dauern vertretenden Klassen ebenfalls gleich oder etwas höher besetzt. Am stärksten wirkt sich dic Vorverlegung des Verfahrensbeginns auf die erste Zeitspanne aus. Beim GAA-N wie beim LRA-1 wurden nunmehr nicht mehr ein Viertel, sondern nur noch ein Fünftel aller Verfahren innerhalb von drei Monaten (20,7\% bzw. 20,9\%) abgeschlossen.

Den geringen Verschiebungen in den Häufigkeitsverteilungen entsprechen nur leichte Erhöhungen der durchschnittlichen Verfahrensdauer (vgl. die Werte in Tab. 37.11 gegenüber denen in Tab. 36.11). ${ }^{339}$ Beim GAA-N verlängert sie sich um 0,6 Monate (absolut) von 6,0 auf 6,6 Monate, beim LRA-1 um 0,7 Monate (absolut) von 7,3 auf 8,0 Monate. ${ }^{340}$ Das bedeutet ein Ansteigen bei beiden Ämtern um jeweils rd. 10\%.

338 Die signifikante Differenz $\left(\mathrm{t}=1,50<\mathrm{t}_{(0,95 ; 65)}=1,67\right.$ bei gleichen Varianzen: $\mathrm{F}=$ $\left.2,56<\mathrm{F}_{(0,999 ; 24,41)}=3,0\right)$ bezieht sich auf den Vergleich der Mittelwerte für Änderungsgenehmigungen beim GAA-N bzw. LRA-1.

339 Im übrigen liegt auch bei Vorverlagerung des Verfahrensbeginns von der Unterlagenvollständigkeit auf den Antragseingang die durchschnittliche Gesamtdauer beim LRA-1 weiterhin über der beim GAA-N (8,00 Mon. gegenüber 6,63 Mon.). Auch hier ist die Differenz statistisch nicht signifikant $\left(t=1,084<t_{(0,90 ; 97)}=1,290\right)$, mit der Einschränkung allerdings, daß in diesem Fall die Varianz beim LRA-1 signifikant über der beim GAA-N liegt, selbst unter der Bedingung eines hohen Sicherheitsniveaus für Gleichheit $\left(F=2,52>F_{(0,999 ; 41,56)}=2,31\right)$.

340 Auch die Mediane verschieben sich leicht nach oben (von 4,20 auf 4,66 Monate beim GAA-N und von 4,66 auf 4,87 Monate beim LRA-1). Bei beiden Ämtern sind die Steigerungen der Mittelwerte nicht signifikant (jeweils $t<1,0$ ). 
Tab. 37.1

Verfahrensdauer ab Antragseingang bis Erlaß Genehmigungsbescheid (Häufigkeitsverteilung)

\begin{tabular}{|l|c|c|c|}
\cline { 2 - 4 } \multicolumn{1}{c|}{} & GAA-N & LRA-1 & LRA-2 \\
\hline \multirow{2}{*}{ Bis zu drei Monaten } & 12 & 9 & 5 \\
& $20,7 \%$ & $20,9 \%$ & $20,8 \%$ \\
\hline \multirow{2}{*}{ Über drei bis sechs Monate } & 24 & 18 & 9 \\
& $41,4 \%$ & $41,9 \%$ & $37,5 \%$ \\
\hline \multirow{2}{*}{ Über sechs bis zwölf Monate } & 10 & 8 & 6 \\
\hline \multirow{2}{*}{ Über zwölf Monate* } & $17,2 \%$ & $18,6 \%$ & $25,0 \%$ \\
\hline \hline \multirow{2}{*}{ Verfahren gesamt } & 12 & 8 & 4 \\
\hline
\end{tabular}

* Maximaler Wert beim GAA-N: 36,3 Mon., LRA-1: 50,4 Mon., LRA-2: 20,1 Mon.

Verantwortlich dafür, daß sich die Vorverlegung des Verfahrensbeginns vom Zeitpunkt der Unterlagenvollständigkeit auf den des Antragseingangs nicht stärker in einer Verlängerung der Gesamtdauer niederschlägt, ist der Umstand, daß die Genehmigungsbehörden nur in der Minderheit der Verfahren bei der Unterlagenprüfung zum Ergebnis eines Ergänzungsbedarfs gekommen sind. Wie erwähnt (Ziff. 2.1, Tab. 12), war dies beim GAA-N lediglich in $32,8 \%$ und beim LRA-1 sogar nur in 16,3\% der Anträge der Fall. Wenn aus dieser unterschiedlichen Ausgangslage bei den beiden Ämtern gleichwohl ein in etwa gleich hoher Anstieg der Gesamtdauer resultiert, verweist das darauf, daß das LRA-1 mehr Zeit aufbrachte (oder aufbringen mußte) als das GAA-N, um den Antragsteller zur Komplettierung der nachgeforderten Unterlagen zu bewegen. Dies wiederum ließe auf ein größeres Durchsetzungspotential der niedersächsischen Behörde gegenüber säumigen Betreibern schließen.

Die mit dem vorverlegten Verfahrensbeginn einhergehende, insgesamt aber eher geringe Verfahrensverlängerung betrifft Neu- und Änderungsgenehmigungen gleichermaßen. Das zeigt der Vergleich der jeweiligen Häufigkeitsverteilungen (Tab. $37.2 \mathrm{zu}$ Tab. 36.2) und gilt grundsätzlich auch für die jeweiligen durchschnittlichen Dauern ${ }^{341}$ (Tab. $37.21 \mathrm{zu}$ Tab. 36.21). ${ }^{342}$

341 Die jeweiligen Mittelwertunterschiede (und die Differenzen der zugehörigen Varianzen) sind bei beiden Behörden nicht signifikant (jeweils $t<1,0$ ). 
Tab. 37.11

Verfahrensdauer ab Antragseingang bis Erlaß Genehmigungsbescheid (statistische Parameter in Monaten)*

\begin{tabular}{|l|c|c|c|}
\cline { 2 - 4 } \multicolumn{1}{c|}{} & GAA-N & LRA-1 & LRA-2 \\
\hline Arith. Mittel & 6,63 & 8,00 & 6,50 \\
\hline Varianz & 23,39 & 59,00 & 15,76 \\
\hline Median & 4,66 & 4,87 & 5,51 \\
\hline Minimum & 0,82 & 1,41 & 1,97 \\
\hline Maximum & 20,07 & 31,70 & 15,31 \\
\hline \hline Gesamt & 57 & 42 & 23 \\
\hline
\end{tabular}

* Ohne Extremwerte.

342 Allerdings ergibt sich ein Unterschied zwischen den beiden Modellen insofern, als die größere Steigerungsrate beim GAA-N auf die Neugenehmigungen, beim LRA1 dagegen auf die Änderungsgenehmigungen entfällt. Beim GAA-N steigen die Mittelwerte bei den Änderungsgenehmigungen um (absolut) 0,54 Mon. (=9,0\%) von 5,97 auf 6,51 Mon., bei den Neugenehmigungen dagegen um (absolut) 0,80 Mon. (=13,0\%) von 6,15 auf 6,95 Mon. Beim LRA-1 steigen sie bei den Änderungsgenehmigungen um (absolut) 1,12 Mon. (= 13,6\%) von 8,27 auf 9,39 Mon., bei den Neugenehmigungen nur um (absolut) 0,14 Mon. (=2,3\%) von 5,81 auf 5,94 Mon. Die divergenten Relationen dürften in erster Linie darauf beruhen, daß die Fälle mit Vervollständigungsbedarf der Unterlagen beim GAA-N vor allem Änderungsgenehmigungen betrafen (zu zwei Dritteln), beim LRA-1 dagegen gleichermaßen Änderungs- wie Neugenehmigungen. Wegen der geringen absoluten Anzahl der einschlägigen Fälle beim LRA-1 (3 Neu-, 4 Änderungsgenehmigungen) müssen mögliche Gründe (einschließlich der einer bloßen Zufallsverteilung) für diese Ergebnisse offenbleiben. 
Tab. 37.2

Verfahrensdauer ab Antragseingang bis Erlaß Genehmigungsbescheid, getrennt nach Neu- und Änderungsgenehmigungen (Häufigkeitsverteilung)*

\begin{tabular}{|l|c|c|c|c|c|c|}
\cline { 2 - 7 } \multicolumn{1}{c|}{} & \multicolumn{2}{c|}{$G A A-N$} & \multicolumn{2}{c|}{$L R A-1$} & \multicolumn{2}{c|}{ LRA-2 } \\
& Änd.-G & Neu-G & Änd.-G & Neu-G & Änd.-G & Neu-G \\
\hline \multirow{2}{*}{ Bis zu drei Monaten } & $\mathbf{1 0}$ & 2 & $\mathbf{3}$ & 6 & $\mathbf{3}$ & 2 \\
& $\mathbf{2 3 , 8 \%}$ & $12,5 \%$ & $\mathbf{1 1 , 5 \%}$ & $35,3 \%$ & $\mathbf{2 5 , 0 \%}$ & $16,7 \%$ \\
\hline \multirow{2}{*}{ Über drei bis sechs Monate } & $\mathbf{1 8}$ & 6 & $\mathbf{1 3}$ & 5 & $\mathbf{4}$ & 5 \\
& $\mathbf{4 2 , 9 \%}$ & $37,5 \%$ & $\mathbf{5 0 , 0 \%}$ & $29,4 \%$ & $\mathbf{3 3 , 3 \%}$ & $41,7 \%$ \\
\hline \multirow{2}{*}{ Über sechs bis zwölf Monate } & $\mathbf{6}$ & 4 & $\mathbf{3}$ & 5 & $\mathbf{1}$ & $\mathbf{5}$ \\
& $\mathbf{1 4 , 3 \%}$ & $25,0 \%$ & $\mathbf{1 1 , 5 \%}$ & $29,4 \%$ & $\mathbf{8 , 3 \%}$ & $41,7 \%$ \\
\hline \multirow{2}{*}{ Über zwölf Monate } & $\mathbf{8}$ & 4 & $\mathbf{7}$ & 1 & $\mathbf{4}$ & 0 \\
\hline \hline \multirow{2}{*}{ Verfahren gesamt** } & $\mathbf{1 9 , 0 \%}$ & $25,0 \%$ & $\mathbf{2 6 , 9 \%}$ & $5,9 \%$ & $\mathbf{3 3 , 3 \%}$ & $0,0 \%$ \\
\hline
\end{tabular}

* Angesichts der teilweise niedrigen absoluten Grundgesamtheiten dient die Prozentuierung lediglich der näherungsweisen (heuristischen) Vergleichbarkeit der auf die einzelnen Kategorien entfallenden absoluten Häufigkeiten.

** Abweichungen von 100,0\% durch Rundungen bei den prozentualen Einzelhäufigkeiten.

Tab. 37.21

Verfahrensdauer ab Antragseingang bis Erlaß Genehmigungsbescheid, getrennt nach Neu- und Änderungsgenehmigungen (statistische Parameter in Monaten)*

\begin{tabular}{|l|c|c|c|c|c|c|}
\cline { 2 - 8 } \multicolumn{1}{c|}{} & \multicolumn{2}{c|}{$G A A-N$} & \multicolumn{2}{c|}{$L R A-1$} & \multicolumn{2}{c|}{$L R A-2$} \\
\cline { 2 - 8 } & Änd.-G & Neu-G & Änd.-G & Neu-G & Änd.-G & Neu-G \\
\hline Arith. Mittel & $\mathbf{6 , 5 1}$ & 6,96 & $\mathbf{9 , 3 9}$ & 5,94 & $\mathbf{6 , 8 0}$ & 6,23 \\
\hline Varianz & $\mathbf{2 4 , 9 1}$ & 20,46 & $\mathbf{7 6 , 7 4}$ & 28,54 & $\mathbf{2 6 , 1 4}$ & 7,58 \\
\hline Median & $\mathbf{4 , 3 5}$ & 5,11 & $\mathbf{5 , 0 2}$ & 3,70 & $\mathbf{5 , 2 5}$ & 5,54 \\
\hline Minimum & $\mathbf{0 , 8 2}$ & 1,74 & $\mathbf{2 , 1 0}$ & 1,41 & $\mathbf{1 , 9 7}$ & 2,13 \\
\hline Maximum & $\mathbf{2 0 , 0 7}$ & 16,59 & $\mathbf{3 1 , 7 0}$ & 22,95 & $\mathbf{1 5 , 3 1}$ & 10,13 \\
\hline \hline Gesamt & $\mathbf{4 2}$ & 15 & $\mathbf{2 5}$ & 17 & $\mathbf{1 1}$ & 12 \\
\hline
\end{tabular}

* Ohne Extremwerte. 


\subsubsection{Dauer ab erster Vorantragsberatung bis zum Erlaß des Genehmigungsbescheids}

Läßt man das Verfahren nicht erst mit dem Antragseingang, sondern bereits mit dem Zeitpunkt des allerersten Kontakts der Genehmigungsbehörde mit dem (künftigen) Antragsteller beginnen, schließt man also die »inoffizielle « Phase mit ein, in der sich die beiden Hauptbeteiligten tatsächlich schon mit der Genehmigungsmaterie befassen, ${ }^{343}$ führt dies zu einer weiteren Erhöhung der (rechnerischen) Gesamtdauer unter dem vergleichenden Gesichtspunkt verschiedener Anfangszeitpunkte. ${ }^{344}$ Auf das Gesamtbild der Verfahrensdauern bei den beiden Genehmigungsmodellen wirkt sich die neuerliche Vorverlagerung des Verfahrensbeginns naheliegenderweise umso stärker aus, je mehr Verfahren mit hier als Vorantragsberatungen konkretisierten Kontakten anfallen. Wie gezeigt (Ziff. 1.3.1, Tab. 8), fanden bei der großen Mehrheit der Verfahren solche Beratungen statt, beim LRA-1 mit einem Anteil von rd. 90\% noch etwas häufiger als beim GAA-N mit einem Anteil von gut $80 \%$.

Tab. 38.1 zeigt, wie sich die Verfahren auf die verschiedenen Klassen von Gesamtdauern bei Anrechnung auch der »inoffiziellen « Bearbeitungszeiten verteilen. Im Vergleich zur Gesamtdauer ab Antragseingang, die bei beiden Genehmigungsmodellen in güt $60 \%$ der Verfahren die Sechsmonatsfrist nicht überstieg (vgl. oben Ziff. 4.2.2.2, Tab. 37.1), ist das nunmehr beim LRA-1 lediglich in 30,2\% der Verfahren, beim GAA-N sogar nur in 19,0\% der Verfahren der Fall (Kumulation der ersten beiden Kategorien der Tabelle). Der Anteil der Verfahren, die sich innerhalb von drei Monaten erledigten, sinkt von jeweils rd. $20 \%$ auf weniger als $10 \%$ (GAA-N: $8,6 \%$, LRA-1: 4,7\%). Auf der anderen Seite finden sich jetzt erheblich mehr Verfahren in den oberen Häufigkeitsklassen. Dauerten unter der Berechnung ab Antragseingang bei beiden Ämtern rd. 20\% der Verfahren länger als 12 Monate, liegt der Anteil jetzt beim GAA-N bei $41,4 \%$ und beim LRA-1 bei $58,1 \%$ (Kumulation der letzten beiden Kategorien der Tabelle).

$343 \mathrm{Zu}$ den Bedenken, diese Vorantragsphase von der restlichen Verfahrenslaufzeit als lediglich »inoffizielle« zu separieren, vgl. oben Ziff. 4.2.1, Buchst. c).

344 Der auf die Berechnungsgrundlage bezogene Vergleichsgesichtspunkt ist deshalb zu betonen, um der verfehlten Schlußfolgerung vorzubeugen, Vorantragsberatungen erhöhten per se die Gesamtverfahrensdauer. Dies ist freilich nicht von vornherein auszuschließen. Ebensogut ist es aber möglich, daß sie die Gesamtdauer im Ergebnis verkürzen, weil sie den offiziellen Teil des Verfahrens entlasten. Darauf wird im einzelnen weiter unten (Ziff. 4.3.1) zurückzukommen sein. 
Tab. 38.1

Verfahrensdauer ab erster Vorantragsberatung bis Erlaß Genehmigungsbescheid (Häufigkeitsverteilung)

\begin{tabular}{|l|c|c|c|}
\cline { 2 - 4 } \multicolumn{1}{c|}{} & GAA-N & LRA-1 & LRA-2 \\
\hline Bis zu drei Monaten & 5 & 2 & 2 \\
& $8,6 \%$ & $4,7 \%$ & $8,3 \%$ \\
\hline Über drei bis sechs Monate & 6 & 11 & 4 \\
\hline Über sechs bis zwölf Monate & $10,3 \%$ & $25,6 \%$ & $16,7 \%$ \\
\hline Über zwölf Monate bis & 23 & 5 & 8 \\
vierundzwanzig Monate & $39,7 \%$ & $11,6 \%$ & $33,3 \%$ \\
\hline Über vierundzwanzig Monate* & 17 & 13 & 8 \\
\hline \hline Verfahren gesamt** & $29,3 \%$ & $30,2 \%$ & $33,3 \%$ \\
\hline \hline
\end{tabular}

* Maximaler Wert beim GAA-N: 38,7 Mon., LRA-1: 76,1 Mon., LRA-2: 29,8 Mon.

** Abweichungen von $100,0 \%$ durch Rundungen bei den prozentualen Einzelhäufigkeiten.

Zusammengefaßt führt also der Einschluß der Vorantragsphase dazu, daß die für den Verfahrensbeginn ab Antragszeitraum ermittelte weitgehende Ähnlichkeit zwischen den beiden Genehmigungsmodellen in der Verteilung der Verfahren auf die verschiedenen Zeitklassen größeren Unterschieden weicht. Im Kern laufen sie darauf hinaus, daß beim LRA-1 gegenüber dem GAA-N einerseits mehr Verfahren in kürzeren Dauern bis zu sechs Monaten zu Ende gebracht wurden (30,2\% gegenüber 19,0\%), andererseits aber auch mehr Verfahren eine »überlange « Dauer von mehr als 24 Monaten, also von mehr als zwei Jahren, beanspruchten $(27,9 \%$ gegenüber $12,1 \%)^{345}$

Es ist weniger der Umstand eines etwas größeren Anteils an Verfahren mit Vorantragsberatungen als vielmehr die vergleichsweise hohe Anzahl an besonders langwierigen Verfahren, die die durchschnittliche Dauer beim LRA-1 deutlich über die beim GAA-N anwachsen 1 äßt, ${ }^{346}$ und zwar auf 16,5 Monate hier gegenüber 11,9 Monaten dort (Tab. 38.11 zu Tab. 37.11). Die Differenz von 4,6 Monaten in der Gesamtdauer ab der ersten Voran-

345 Während die erstgenannte Differenz (Anteile der Verfahren bis 6 Mon.) statistisch nicht signifikant ist $\left(\mathrm{chi}^{2}=1,73<2,71\right.$ für $\mathrm{p}=0,1$ und $\left.\mathrm{df}=1\right)$, ist das bei der zweiten eindeutig der Fall $\left(\mathrm{chi}^{2}=4,05>3,84, \mathrm{p}=0,05, \mathrm{df}=1\right)$.

$346 \mathrm{Vgl}$. dazu im einzelnen unten Ziff. 4.3.1. 
tragsberatung ist auf einem Wahrscheinlichkeitsniveau von 99\% statistisch signifikant. ${ }^{347}$ In ähnlicher Größenordnung wie die Mittelwerte divergieren auch die Mediane, und zwar um 5,0 Monate (15,1 zu 10,1 Mon. beim LRA1 bzw. GAA-N).

Tab. 38.11

Verfahrensdauer ab erster Vorantragsberatung bis Erlaß Genehmigungsbescheid (statistische Parameter in Monaten) ${ }^{*}$

\begin{tabular}{|l|c|c|c|}
\cline { 2 - 4 } \multicolumn{1}{c|}{} & GAA-N & LRA-1 & LRA-2 \\
\hline Arith. Mittel & 11,86 & 16,46 & 10,81 \\
\hline Varianz & 55,04 & 136,48 & 35,23 \\
\hline Median & 10,11 & 15,08 & 10,77 \\
\hline Minimum & 1,74 & 2,56 & 2,13 \\
\hline Maximum & 33,38 & 40,42 & 21,84 \\
\hline \hline Gesamt & 56 & 41 & 22 \\
\hline
\end{tabular}

* Ohne Extremwerte.

Im Vergleich zur Berechnung ab Antragseingang ist die mittlere Verfahrenszeit beim LRA-1 von 8,0 Monaten um 8,5 Monate $(=105,8 \%)$ auf etwas mehr als das Doppelte gestiegen und damit deutlich stärker als beim GAA$\mathrm{N}$, wo sie sich von 6,6 Monaten für sich genommen ebenfalls erheblich, vergleichsweise aber »nur« um 5,2 Monate $(=78,9 \%)$ erhöhte. ${ }^{348}$

Als Ergebnis läßt sich festhalten, daß das Bild über den Zeitbedarf der Genehmigungspraxis entscheidend davon beeinflußt wird, ob es auf der Basis der tatsächlichen »Anhängigkeit« eines Verfahrens bei der Genehmigungsbehörde oder auf der Basis eines offiziell bestimmten Anfangszeitpunkts gezeichnet wird. Zugespitzt formuliert: Die offizielle Verfahrensphase macht in etwa nur die Hälfte der tatsächlich für die Genehmigungs-

$347 \mathrm{t}=2,370>\mathrm{t}_{(0,99 ; 95)}=2,364$. Die erhebliche Differenz der Varianzen $(136,477 \mathrm{ge}-$ genüber 55,035) ist allerdings nur bei einem hohen Sicherheitsniveau für die Annahme von Ungleichheit nicht signifikant $\left(F=2,48<\mathrm{F}_{(0,999 ; 40,55)}=2,50\right)$.

348 Bei beiden Ämtern ist der Anstieg der Verfahrensdauer hochsignifikant (LRA-1: t $=3,909>\mathrm{t}_{(0,9995 ; 81)}=3,415$ bei $\mathrm{F}=2,31<\mathrm{F}_{(0,999 ; 40,41)}=2,75 ;$ GAA-N: $\mathrm{t}=4,447$ $>\mathrm{t}_{(0,9995 ; 111)}=3,390$ bei $\left.\mathrm{F}=2,35<\mathrm{F}_{(0,999 ; 55,56)}=2,38\right)$. 
erteilung in Anspruch genommenen Zeitspanne sichtbar. Das gilt im Grunde unabhängig vom jeweiligen Genehmigungsmodell. ${ }^{349}$

Tab. 38.2

Verfahrensdauer ab erster Vorantragsberatung bis Erlaß Genehmigungsbescheid, getrennt nach Neu- und Änderungsgenehmigungen (Häufigkeitsverteilung) ${ }^{*}$

\begin{tabular}{|c|c|c|c|c|c|c|}
\hline & \multicolumn{2}{|c|}{$G A A-N$} & \multicolumn{2}{|c|}{$L R A-1$} & \multicolumn{2}{|c|}{$L R A-2$} \\
\hline & Änd.-G & $N e u-G$ & Änd.-G & Neu-G & Änd.-G & $\mathrm{Neu}-\mathrm{G}$ \\
\hline Bis zu drei Monaten & $\stackrel{4}{9,5 \%}$ & $\begin{array}{c}1 \\
6,3 \%\end{array}$ & $\begin{array}{c}1 \\
3,8 \%\end{array}$ & $\begin{array}{c}1 \\
5,9 \%\end{array}$ & $\begin{array}{c}1 \\
8,3 \%\end{array}$ & $\begin{array}{c}1 \\
8,3 \%\end{array}$ \\
\hline Über drei bis sechs Monate & $\begin{array}{c}4 \\
9,5 \%\end{array}$ & $\begin{array}{c}2 \\
12,5 \%\end{array}$ & $\begin{array}{c}4 \\
15,4 \%\end{array}$ & $\begin{array}{c}7 \\
41,2 \%\end{array}$ & $\begin{array}{c}1 \\
8,3 \%\end{array}$ & $\begin{array}{c}3 \\
25,0 \%\end{array}$ \\
\hline Über sechs bis zwölf Monate & $\begin{array}{c}19 \\
45,2 \%\end{array}$ & $\begin{array}{c}4 \\
25,0 \%\end{array}$ & $\begin{array}{c}4 \\
15,4 \%\end{array}$ & $\begin{array}{c}1 \\
5,9 \%\end{array}$ & $\begin{array}{c}3 \\
25,0 \%\end{array}$ & $\begin{array}{c}5 \\
41,7 \%\end{array}$ \\
\hline $\begin{array}{l}\text { Über zwölf Monate bis } \\
\text { vierundzwanzig Monate }\end{array}$ & $\begin{array}{c}10 \\
23,8 \%\end{array}$ & $\begin{array}{c}7 \\
43,8 \%\end{array}$ & $\stackrel{9}{34,6 \%}$ & $\begin{array}{c}4 \\
23,5 \%\end{array}$ & $\begin{array}{c}6 \\
50,0 \%\end{array}$ & $\begin{array}{c}2 \\
16,7 \%\end{array}$ \\
\hline Über vierundzwanzig Monate & $\begin{array}{c}5 \\
11,9 \%\end{array}$ & $\begin{array}{c}2 \\
12,5 \%\end{array}$ & $\begin{array}{c}8 \\
30,8 \%\end{array}$ & $\begin{array}{c}4 \\
23,5 \%\end{array}$ & $\begin{array}{c}1 \\
8,3 \%\end{array}$ & $\begin{array}{c}1 \\
8,3 \%\end{array}$ \\
\hline Verfahren gesamt** & $\begin{array}{c}42 \\
99,9 \%\end{array}$ & $\begin{array}{c}16 \\
100,1 \%\end{array}$ & $\begin{array}{c}26 \\
100,0 \%\end{array}$ & $\begin{array}{c}17 \\
100,0 \%\end{array}$ & $\begin{array}{c}12 \\
99,9 \%\end{array}$ & $\begin{array}{c}12 \\
100,0 \%\end{array}$ \\
\hline
\end{tabular}

* Angesichts der teilweise niedrigen absoluten Grundgesamtheiten dient die Prozentuierung lediglich der näherungsweisen (heuristischen) Vergleichbarkeit der auf die einzelnen Kategorien entfallenden absoluten Häufigkeiten.

** Abweichungen von $100,0 \%$ durch Rundungen bei den prozentualen Einzelhäufigkeiten.

Die zusätzliche Differenzierung der die Vorantragsphase einbeziehenden Gesamtdauer nach Neu- und Änderungsgenehmigungen, die Tab. 38.2 nach Zeitklassen und Tab. 38.21 nach statistischen Parametern ausweist, legt einen graduellen Unterschied zwischen der baden-württembergischen und niedersächsischen Genehmigungsbehörde offen, der sich bereits bei der Dauer ab Antragseingang - in abgeschwächter Form - gezeigt hat. ${ }^{350}$

$349 \mathrm{Daß}$ die Vorverhandlungen »etwa so lange dauern« wie die anschließenden formellen Verfahren, konstatiert schon die frühe Untersuchung von Mayntz et al. 1978, S. 35, die sich allerdings in erster Linie auf schriftliche Befragungen der Vollzugsbehörden stützt.

350 Vgl. oben Ziff. 4.2.2.2, Anm. 342. 
Während sich beim GAA-N die kürzeren Dauern bis zu sechs Monaten (Kumulation der ersten beiden Kategorien in Tab. 38.2) und die besonders langen Dauern von über 24 Monaten nahezu gleichmäßig auf Neu- und Änderungsgenehmigungen verteilen (mit einem leichten Übergewicht der Neugenehmigungen zugunsten längerer Dauern), sind es beim LRA-1 vor allem die Änderungsgenehmigungen, die weniger mit kürzeren und häufiger mit besonders langen Dauern und entsprechend auch mit einem (wenngleich nicht signifikant) höheren Durchschnittswert einhergingen (Tab. 38.21). Dieser Unterschied gründet in erster Linie, wie die nachfolgenden Befunde zur Dauer einzelner Verfahrensabschnitte zeigen werden, nicht in der Vorantragsphase, sondern in der »offiziellen« Laufzeit.

Tab. 38.21

Verfahrensdauer ab erster Vorantragsberatung bis Erlaß Genehmigungsbescheid, getrennt nach Neu- und Änderungsgenehmigungen (statistische Parameter in Monaten)*

\begin{tabular}{|l|r|r|r|r|r|r|}
\cline { 2 - 7 } & \multicolumn{2}{c|}{$G A A-N$} & \multicolumn{2}{c|}{$L R A-I$} & \multicolumn{2}{c|}{ LRA-2 } \\
\cline { 2 - 7 } & Änd.-G & Neu-G & Änd.-G & Neu-G & Änd.-G & Neu-G \\
\hline Arith. Mittel & $\mathbf{1 1 , 6 0}$ & 12,56 & $\mathbf{1 8 , 1 5}$ & 13,82 & $\mathbf{1 2 , 6 2}$ & 9,00 \\
\hline Varianz & $\mathbf{5 6 , 5 0}$ & 54,07 & $\mathbf{1 2 1 , 9 5}$ & 156,67 & $\mathbf{4 0 , 3 4}$ & 26,44 \\
\hline Median & $\mathbf{9 , 7 1}$ & 12,39 & $\mathbf{1 7 , 0 2}$ & 6,66 & $\mathbf{1 2 , 7 6}$ & 8,88 \\
\hline Minimum & $\mathbf{1 , 8 7}$ & 1,74 & $\mathbf{2 , 7 8}$ & 2,56 & $\mathbf{2 , 5 3}$ & 2,13 \\
\hline Maximum & $\mathbf{3 3 , 3 8}$ & 28,07 & $\mathbf{4 0 , 4 2}$ & 39,04 & $\mathbf{2 1 , 8 4}$ & 20,23 \\
\hline \hline Gesamt & $\mathbf{4 1}$ & 15 & $\mathbf{2 5}$ & 16 & $\mathbf{1 1}$ & 11 \\
\hline
\end{tabular}

* Ohne Extremwerte.

\subsection{Dauern einzelner Verfahrensabschnitte}

Die Aufgliederung der Gesamtdauer in verschiedene Verfahrensabschnitte gibt Aufschluß darüber, ob bzw. in welchem Maße bestimmte Phasen den Verfahrensablauf besonders belasten. 


\subsubsection{Vorantragsberatung (»Vorphase«)}

Die große Mehrheit der Verfahren wurde, wie verschiedentlich erwähnt, in beiden Genehmigungsmodellen im Wege »inoffizieller « ${ }^{351}$ Vorantragsberatungen eingeleitet. Tab. 39 zeigt, wie sich diese vorgeschalteten Beratungsaktivitäten auf verschiedene Zeitklassen verteilen, Tab. 39.1 enthält die zugehörigen statistischen Parameter.

Tab. 39

Dauer der Vorantragsberatung (Zeitspanne zwischen erster behördlicher Beratung und Antragseingang) (Häufigkeitsverteilung)

\begin{tabular}{|l|c|c|c|}
\cline { 2 - 4 } \multicolumn{1}{c|}{} & GAA-N & $L R A-1$ & $L R A-2$ \\
\hline Bis zu drei Monaten & 8 & 11 & 8 \\
& $16,7 \%$ & $28,2 \%$ & $42,1 \%$ \\
\hline Über drei bis sechs Monate & 19 & 8 & 4 \\
& $39,6 \%$ & $20,5 \%$ & $21,1 \%$ \\
\hline Über sechs bis zwölf Monate & 15 & 8 & 3 \\
\hline Über zwölf Monate* & $31,3 \%$ & $20,5 \%$ & $15,8 \%$ \\
\hline \hline Verfahren mit Beratung gesamt & 6 & 12 & 4 \\
\hline
\end{tabular}

* Maximaler Wert beim GAA-N: 23,9 Mon., LRA-1: 36,5 Mon., LRA-2: 26,2 Mon.

** Abweichungen von $100,0 \%$ durch Rundungen bei den prozentualen Einzelhäufigkeiten.

Nach Tab. 39 nahmen die Vorantragsberatungen beim GAA-N in etwas mehr und beim LRA-1 in etwas weniger als der Hälfte $(56,3 \%$ bzw. 48,7\%) der einschlägigen Verfahren maximal sechs Monate in Anspruch (Kumulation der ersten beiden Zeitkategorien). Dabei weist das LRA-1 gegenüber dem GAA-N sogar einen höheren Anteil an Verfahren auf, in denen die »Vorphase « bereits binnen dreier Monate zum Abschluß gebracht werden konnte $(28,2 \%$ gegenüber $16,7 \%){ }^{352}$ Signifikant unterscheiden sich die Ämter allerdings in den Anteilen von Vorantragsberatungen, die sich über mehr als 12 Monate hinzogen. Beim GAA-N sind es 12,5\%, beim LRA-1

351 Zur Problematik der Verwendung dieses Begriffs im vorliegenden Zusammenhang vgl. oben Ziff. 4.2.1, Buchst. c.

352 Die Differenz ist jedoch nicht signifikant $\left(\operatorname{chi}^{2}=1,67<2,71\right.$ für $\mathrm{p}=0,1$ und $\left.\mathrm{df}=1\right)$. 
dagegen $30,8 \% \cdot{ }^{353}$. Bei der baden-württembergischen Behörde kommt hinzu, daß die Hälfte der Verfahren dieser Kategorie mit einer Beratungsphase von über 24 Monaten einherging. Die niedersächsische Behörde hat dagegen keinen einzigen Fall mit einer solchen Dauer zu verbuchen. ${ }^{354}$

»Überlange« Vorantragszeiten sind, wie eine nähere Betrachtung ergibt, beim LRA-1 in erster Linie einer höheren Beratungsintensität mit einer Mehrzahl von Terminen (bis zu 20) geschuldet und weniger einer Diskontinuität der Beratungsaktivitäten (zeitweiliger »Leerlauf« oder Untätigkeit der Behörde). Das deutet darauf hin, daß die baden-württembergische Genehmigungsbehörde größere Schwierigkeiten hatte als die niedersächsische, sich gegenüber dem Antragsteller mit ihren Forderungen im Hinblick auf einen genehmigungsfähigen Antrag durchzusetzen. Auf der anderen Seite wurden die »überlangen« Vorantragsdauern beim LRA-1 nahezu durchweg mit einer vergleichsweisen kurzen Dauer von - zum Teil deutlich - unter sechs Monaten der darauffolgenden »offiziellen« Verfahrenszeit ab Antragseingang kompensiert, was beim GAA-N nicht der Fall ist. Vielmehr schlossen sich dort an die »überlangen« Vorantragsberatungen zumeist nicht minder lange »offizielle« Verfahrenszeiten von 12 und mehr Monaten an.

Für das günstigere Abschneiden der baden-württembergischen Behörde bei der offiziellen Verfahrensdauer in den Fällen, die sich in der Vorantragsphase als besonders »schwierig « erwiesen haben bzw. als solche wahrgenommen wurden, käme als Erklärung nicht nur die umfänglichere, rein quantitative Intensität der Beratung in Betracht. Zu denken wäre an eine zudem auch höhere Qualität der Beratung. Das Beratungsniveau wurde zwar hier nicht gemessen und muß insofern prinzipiell offenbleiben. Als ein Indikator für die Vermutung einer höheren Qualität ließe sich aber der Befund betrachten, daß das LRA-1 deutlich häufiger als das GAA-N andere, in ihren Belangen betroffene Fachbehörden bereits zu den Vorantragsberatungen zuzog (wie es $\S 2$ Abs. 2 Satz 2 der 9. BImSchV gestattet). ${ }^{355} \mathrm{Im} \mathrm{Er-}$ gebnis kann es so in gewissem Maße die Behördenbeteiligung vom Antrags- auf den Vorantragszeitraum vorverlagern. Dieser Effekt wäre insofern modellbedingt, als gerade in »schwierigen « Fällen die eingeschränkte Kompetenz in fachtechnischen Fragen einer auf Verwaltungsaufgaben spezialisierten Behörde an ihre Grenzen stößt. In der Gesamtheit der Verfahren wird beim LRA-1 die gegenüber dem GAA-N durchschnittlich längere Vor-

$353 \mathrm{chi}^{2}=4,37>3,84, \mathrm{p}=0,05, \mathrm{df}=1$.

354 Siehe auch den jeweiligen Maximalwert der Verteilungen, der beim LRA-1 36,5 Monate, beim GAA-N aber nur 23,9 Monate beträgt.

355 Vgl. oben Ziff. 1.3.2. - Eine frühe Beteiligung von Fachbehörden kann auch Ausdruck der Strategie sein, diese in den Entscheidungsprozeß einzubinden. 
antragsphase allerdings nicht durch entsprechend kürzere Dauern der »offiziellen« Laufzeit kompensiert. ${ }^{356}$

Tab. 39.1

Dauer der Vorantragsberatung (Zeitspanne zwischen erster behördlicher Beratung und Antragseingang) (statistische Parameter in Monaten) ${ }^{*}$

\begin{tabular}{|l|c|c|c|}
\cline { 2 - 4 } \multicolumn{1}{c|}{} & GAA-N & LRA-1 & LRA-2 \\
\hline Arith. Mittel & 6,77 & 10,56 & 6,64 \\
\hline Varianz & 24,06 & 120,32 & 55,77 \\
\hline Median & 5,43 & 6,98 & 4,03 \\
\hline Minimum & 0,82 & 0,03 & 0,26 \\
\hline Maximum & 23,90 & 36,52 & 26,23 \\
\hline \hline Gesamt & 48 & 39 & 19 \\
\hline
\end{tabular}

* Ohne Extremwerte.

Tab. 39.2

Dauer der Vorantragsberatung (Zeitspanne zwischen erster behördlicher Beratung und Antragseingang), getrennt nach Neu- und Änderungsgenehmigungen (statistische Parameter in Monaten) ${ }^{*}$

\begin{tabular}{|l|r|r|r|r|r|r|}
\cline { 2 - 8 } & \multicolumn{2}{c|}{ AAA-N } & \multicolumn{2}{c|}{$L R A-1$} & \multicolumn{2}{c|}{$L R A-2$} \\
& Änd.-G & Neu-G & Änd.-G & Neu-G & Änd.-G & Neu-G \\
\hline Arith. Mittel & $\mathbf{6 , 6 9}$ & 7,01 & $\mathbf{1 0 , 1 8}$ & 11,16 & $\mathbf{6 , 5 7}$ & 6,75 \\
\hline Varianz & $\mathbf{2 6 , 7 2}$ & 17,71 & $\mathbf{9 4 , 6 0}$ & 170,52 & $\mathbf{6 2 , 9 6}$ & 53,45 \\
\hline Median & $\mathbf{5 , 3 3}$ & 5,89 & $\mathbf{7 , 1 5}$ & 3,05 & $\mathbf{3 , 1 5}$ & 4,21 \\
\hline Minimum & $\mathbf{0 , 8 2}$ & 1,54 & $\mathbf{0 , 0 3}$ & 0,62 & $\mathbf{0 , 5 6}$ & 0,26 \\
\hline Maximum & $\mathbf{2 3 , 9 0}$ & 14,52 & $\mathbf{3 4 , 9 8}$ & 36,52 & $\mathbf{2 6 , 2 3}$ & 21,15 \\
\hline \hline Gesamt & $\mathbf{3 6}$ & 12 & $\mathbf{2 4}$ & 15 & $\mathbf{1 1}$ & 8 \\
\hline
\end{tabular}

* Ohne Extremwerte.

356 S. dazu näher unten Ziff. 4.4 mit Tab. 46. 
Die genannten Divergenzen führen zu deutlich unterschiedlichen Durchschnittswerten der Vorantragsdauer, die beim LRA-1 mit 10,6 Monaten um 3,8 Monate über der beim GAA-N mit 6,8 Monaten liegt (Tab. 39.1). ${ }^{357} \mathrm{Bei}$ beiden Ämtern ist ein Einfluß der Verfahrensart nach Neu- bzw. Änderungsgenehmigungen nicht festzustellen, wie die jeweils nur geringfügigen Abweichungen der mittleren Dauern zeigen (Tab. 39.2).

\subsubsection{Vollständigkeitsprüfung der Antragsunterlagen}

Die für die untersuchten Verfahren gültige Gesetzeslage verpflichtete die Genehmigungsbehörde zwar, die Antragsunterlagen »unverzüglich« nach Eingang auf deren Vollständigkeit hin zu prüfen. Eine ausdrückliche Frist für diesen Arbeitsgang war ihr jedoch nicht gesetzt. Mit der letzten Novelle soll die weiterhin »unverzüglich « vorzunehmende Prüfung nunmehr binnen eines Monats abgeschlossen sein mit der Möglichkeit, die Frist »in begründeten Ausnahmefällen einmal um zwei Wochen« zu verlängern. ${ }^{358}$

Vor dem Hintergrund der alten, aber auch der neuen Gesetzeslage schneiden beide Genehmigungsmodelle in der großen Mehrzahl der Verfahren günstig ab, wie Tab. 40 zeigt.

Beim GAÁ-ĩ wurden mehr ais der Häiffte $(58,6 \%)$ und beim ĽRÁ-1 sogar nahezu vier Fünfteln $(79,1 \%)$ der Antragsteller die Vollständigkeit der eingereichten Unterlagen bereits mit der Eingangsbestätigung de facto attestiert - wenn auch nur in wenigen Ausnahmen ausdrücklich. ${ }^{359} \mathrm{Zu}$ diesen »unverzüglich « erledigten Fällen kommen beim GAA-N weitere 22,4\% und beim LRA-1 weitere 7,0\% hinzu, in denen die Vollständigkeit binnen eines Monats festgestellt wurde. Damit erfolgten bei beiden Genehmigungsmodellen über $80 \%$ der Vollständigkeitskontrollen formell fristgerecht (Kumulation der ersten beiden Kategorien der Tabelle). Lediglich jeweils ein Zehntel der Prüfungen (8,6\% beim GAA-N bzw. 9,3\% beim LRA1) zog sich über mehr als zwei Monate hin und benötigte damit doppelt so viel Zeit wie inzwischen gesetzlich zugestanden. ${ }^{360}$

357 Die Differenz ist auf einem Wahrscheinlichkeitsniveau von 97,5\% signifikant ( $t=$ $\left.2,148>\mathrm{t}_{(0,975 ; 85)}=1,982\right)$, obwohl die Varianzen $(120,315$ gegenüber 24,060$)$ signifikant differieren $\left(\mathrm{F}=5,00>\mathrm{F}_{(0,999 ; 38,47)}=2,60\right)$.

358 Vgl. $\$ 7$ Abs. 1 Satz 1 a.F. und $\S 7$ Abs. 1 Satz 1 u. 2 n.F. der 9. BImSchV.

$359 \mathrm{Vgl}$. ausführlicher dazu oben Ziff. 2.1 und 2.2 mit Anm. 110. 


\section{Tab. 40}

Dauer der Vollständigkeitsprüfung der Antragsunterlagen (Antragseingang bis Vollständigkeit der Unterlagen) (Häufigkeitsverteilung)

\begin{tabular}{|l|c|c|c|}
\cline { 2 - 4 } \multicolumn{1}{c|}{} & $G A A-N$ & $L R A-1$ & $L R A-2$ \\
\hline Vollständigkeit bei Antragseingang & 34 & 34 & 13 \\
& $58,6 \%$ & $79,1 \%$ & $54,2 \%$ \\
\hline \multirow{2}{*}{ Bis zu einem Monat } & 13 & 3 & 3 \\
& $22,4 \%$ & $7,0 \%$ & $12,5 \%$ \\
\hline Über einen bis zwei Monate & 6 & 2 & 3 \\
\hline Über zwei Monate* & $10,3 \%$ & $4,7 \%$ & $12,5 \%$ \\
\hline \hline \multirow{2}{*}{ Verfahren gesamt** } & 5 & 4 & 5 \\
\hline
\end{tabular}

* Maximaler Wert beim GAA-N: 12,0 Mon., LRA-1: 10,1 Mon., LRA-2: 5,3 Mon.

** Abweichungen von 100,0\% durch Rundungen bei den prozentualen Einzelhäufigkeiten.

Insgesamt folgt aus diesen Befunden, daß die zeitliche Belastung der Verfahren durch die Vollständigkeitsprüfung nur wenig ins Gewicht fällt. Das läßt sich auch an ihrer niedrigen durchschnittlichen Dauer ablesen (Tab. 40.1). Beim GAA-N beträgt sie 0,40 Monate, beim LRA-1 0,26 Monate, d.h. bei beiden Ämtern weniger als zwei Wochen. ${ }^{361}$ Angesichts des im ganzen gesehen geringen durchschnittlichen Zeitaufwands kann es nicht überraschen, daß größere Unterschiede zwischen Neu- und Änderungsgenehmigungen nicht auszumachen sind. ${ }^{362}$

Das gezeichnete, für die Genehmigungsbehörden weitgehend vorteilhafte Bild ist freilich zu relativieren, wenn man inhaltliche Anforderungen

360 Durch niedrigere Anteile bei den kürzeren Prüfzeiten (Kumulation der ersten beiden Kategorien in Tab. 40) zugunsten höherer Anteile bei den längeren Prüfzeiten (Kumulation der letzten beiden Kategorien) hebt sich das LRA-2 von den anderen beiden Ämtern deutlich ab (signifikant gegenüber dem LRA-1: $\operatorname{chi}^{2}=3,51>2,71, \mathrm{p}=$ $0,1, \mathrm{df}=1$.). Wegen der bei allen drei Ämtern gegebenen ausgeprägten Asymmetrie der (zudem klassifizierten) Häufigkeitsverteilung durch die starke Besetzung der ersten Kategorie ebnen sich die genannten Anteilsdifferenzen bei den auf den Rohdaten basierenden Durchschnittswerten weitgehend ein (s. nachfolgende Anm.).

361 Die Differenz der Mittelwerte ist nicht signifikant $\left(\mathrm{t}=0,945<\mathrm{t}_{(0,95 ; 95)}=1,661\right.$ bei $\left.\mathrm{F}=1,44<\mathrm{F}_{(0,95 ; 40,55)}=1,61\right)$. Das gilt auch für die Differenz der Mittelwerte zwischen LRA-1 und LRA-2 $\left(\mathrm{t}=1,619<\mathrm{t}_{(0,95 ; 61)}=1,670\right.$ bei $\mathrm{F}=2,25<\mathrm{F}_{(0,99 ; 21,40)}=$ 2,37).

362 Vgl. Tab. 40.2-A im Anhang. 
an die Vollständigkeitsprüfung mit einbezieht. Die Einschränkungen betreffen in besonderem Maße die baden-württembergische Behörde.

Tab. 40.1

Dauer der Vollständigkeitsprüfung der Antragsunterlagen (Antragseingang bis Vollständigkeit der Unterlagen) (statistische Parameter in Monaten)*

\begin{tabular}{|l|c|c|c|}
\cline { 2 - 4 } \multicolumn{1}{c|}{} & GAA-N & LRA-1 & LRA-2 \\
\hline Arith. Mittel & 0,40 & 0,26 & 0,67 \\
\hline Varianz & 0,46 & 0,66 & 1,49 \\
\hline Median & 0,00 & 0,00 & 0,00 \\
\hline Minimum & 0,00 & 0,00 & 0,00 \\
\hline Maximum & 2,72 & 4,10 & 4,52 \\
\hline \hline Gesamt & 56 & 41 & 22 \\
\hline \hline
\end{tabular}

* Ohne Extremwerte.

Vollständigkeit i.S. des $§ 7$ der 9. BImSchV setzt mindestens voraus, daß Antrag und Unterlagen die inhaltlichen, anlagenspezifischen Angaben nach $\S 3$ bzw. $§ 4$ der 9 . BImSchV enthalten. ${ }^{363}$ Wie die oben dargelegten Befunde gezeigt haben (Ziff. 2.4.4 mit Tab. 19), wurden in über einem Drittel der Verfahren beim GAA-N und über zwei Dritteln der Verfahren beim LRA-1 nach »offiziellem « Beginn vom Antragsteller noch weitere Angaben nachgefordert. Ob dieser Ergänzungsbedarf bereits im Rahmen der Vollständigkeitsprüfung hätte erkannt und die entsprechende Komplettierung hätte eingeleitet werden können, kann hier nicht entschieden werden und stünde im Zweifel erst nach einer gerichtlichen Prüfung fest. Die Befunde machen aber immerhin die Problematik des gesetzgeberischen Modells deutlich, die Vollständigkeitsprüfung zu einem »objektiv « begrenzbaren eigenen, dem weiteren Verfahrensgang, insbesondere auch der Fachbe-

363 Vgl. hierzu z.B. die Kommentierung zu $§ 7$ von Czajka 1996, S. 3f. Die Vollständigkeitsprüfung verlangt nach Czajka von der Genehmigungsbehörde nicht, auch die inhaltliche Richtigkeit der Angaben des Antragstellers zu prüfen. Vollständigkeit ist auch dann gegeben, wenn für die Erteilung der Genehmigung noch weiterer Ermittlungsbedarf - etwa durch Einholung eines Sachverständigengutachtens - hinsichtlich der materiellen Genehmigungsvoraussetzungen erkannt wird, entsprechende Ermittlungen dem Antragsteller unter Berücksichtigung des VerhältnismäBigkeitsgrundsatzes aber nicht zuzumuten sind (Ermessen). 
hördenbeteiligung vorgelagerten Stadium zu erheben und sich von einer zeitlichen Disziplinierung gerade dieser, angesichts der Praxis eher künstlich anmutenden Verfahrensphase eine Beschleunigung von Genehmigungen zu versprechen.

Von der angedeuteten grundsätzlichen Problematik einmal abgesehen, erweist sich das niedersächsische Genehmigungsmodell besser gerüstet als das baden-württembergische, den inhaltlichen Anforderungen an die Vollständigkeitsprüfung nachzukommen. Die baden-württembergische Genehmigungsbehörde schob zumeist - den Grenzen ihrer fachtechnischen Kompetenzen Rechnung tragend - die Vollständigkeitsprüfung an das als reine Fachbehörde zuständige GAA ab und leitete damit faktisch zugleich auch das offizielle Verfahren ein. Daß das LRA zur Wahrung seiner Autorität als für das Verfahren verantwortliche Behörde dieses Vorgehen gegenüber dem Antragsteller als »gemeinsame Prüfung der Unterlagen mit GAA« ausgab, ändert nichts daran, daß die Prüfung der Unterlagenvollständigkeit dabei ausschließlich dem GAA überlassen war. Letzteres bekommt so - entsprechend seiner tatsächlichen Stellung im Verfahren - eine herausgehobene Position zugewiesen, denn in bezug auf andere Fachbehörden, bei denen sich für evtl. fachspezifische Nachforderungen die Situation grundsätzlich nicht anders darstellt, unterblieb ein solcher absichernder Vorbehalt.

\subsubsection{Fachbehördenbeteiligung}

Die Fachbehördenbeteiligung stellt nicht nur wegen ihrer faktischen Auswirkungen auf den materiellen Genehmigungsinhalt eine zentrale Phase des Verfahrensablaufs dar. ${ }^{364}$ Auch unter dem hier interessierenden rein zeitlichen Aspekt bildet sie ein separates Stadium. Sie setzt Entscheidungsprozesse autonom agierender Dritter ${ }^{365}$ - gewissermaßen Verfahren im Verfahren - in Gang, deren inhaltliche und in der Folge auch zeitliche Verläufe sich der Verfügung durch die verfahrensleitende Genehmigungsbehörde

364 Vgl. oben Ziff. 2.3, 2.4.2 und 3.1.

365 Neben den Fachbehörden kommen als weitere autonom entscheidende Dritte auch von der Genehmigungsbehörde eingeschaltete Sachverständige zur unabhängigen Begutachtung entscheidungserheblicher Sachverhalte in Betracht ( $\$ 13$ Abs. 1 der 9. BImSchV). Von dieser Möglichkeit wurde in den untersuchten Verfahren allerdings kein Gebrauch gemacht. Dagegen kam die in Abs. 2 der zitierten Bestimmung beschriebene Variante häufiger vor, nach der ein vom Antragsteller eingeholtes Gutachten, dessen Gegenstand mit der Genehmigungsbehörde abgestimmt wurde, einem amtlich veranlaßten gleichgestellt wird. Da in diesem Fall die Initiative vom Antragsteller und nicht von der verfahrensleitenden Instanz ausgeht, werden im vorliegenden Zusammenhang solche Begutachtungen nicht als förmliche Sachverständigenbeteiligung gewertet. 
weitgehend entziehen. ${ }^{366}$ Aus diesem Grunde wird im folgenden - anders als bei der auf den inhaltlichen Verfahrensablauf konzentrierten Analyse das baden-württembergische GAA in die zeitliche Analyse der Fachbehördenbeteiligung mit einbezogen.

\subsubsection{Anforderung der Stellungnahmen durch die Genehmi- gungsbehörde (Fachbehördenbeiziehung)}

Eine ausdrückliche Frist in Form einer quantitativen Zeitvorgabe, innerhalb derer die Genehmigungsbehörde die Fachbehördenbeteiligung einzuleiten hat, schreibt das Gesetz für vereinfachte Verfahren nicht vor. Für die Regelverfahren bestimmt es allerdings, daß die Genehmigungsbehörde »spätestens gleichzeitig « mit der öffentlichen Bekanntmachung des Vorhabens ( $\$ 11 \mathrm{~S} .1$ der 9. BImSchV) die in ihren Belangen betroffenen Fachbehörden zur Abgabe einer Stellungnahme aufzufordern hat. Aus der Verbindung mit der weiteren Vorschrift, nach der die Genehmigungsbehörde das Vorhaben nach Vollständigkeit der erforderlichen Unterlagen öffentlich bekanntzumachen hat ( 8 Abs. 1 Satz 1 der 9. BImSchV), läßt sich ableiten, daß sich die Behördenbeteiligung unmittelbar an den Abschluß der Vollständigkeitsprüfung anzuschließen hat. Der sich so ergebende Zeitpunkt läßt sich auf die vereinfachten Verfahren übertragen, wenn man dem Gesetzgeber Konsistenz in seinen eigenen rechtlichen Vorgaben unterstellt. ${ }^{367}$

Die nachfolgenden Berechnungen zu den Zeiträumen, die zwischen dem Abschluß der Vollständigkeitsprüfung und der (formellen) Einleitung der Fachbehördenbeteiligung in den untersuchten Verfahren verstrichen sind, sind vor dem Hintergrund zu sehen, daß die Genehmigungspraxis nicht durchgängig dem gesetzgeberischen Modell einer zeitgleichen (»sternförmigen«) Beiziehung der Fachbehörden bei der (als Regelfall unterstellten) Beteiligung mehrerer Stellen folgte (vgl. oben Ziff. 4.1). Deshalb wird in einem ersten Schritt die Dauer bis zur zeitlich zuerst einbezogenen Behörde ermittelt (Tab. 41.1 und Tab. 41.11). In einem zweiten Schritt wird dann die Zeitspanne berechnet, die von der Beiziehung des als erstes bis zur Beiziehung des als letztes herangezogenen Fachamts vergangen ist (Tab. 41.2 und Tab. 41.21).

Nach Tab. 41.1 vollzog sich die Einleitung der Behördenbeteiligung im wesentlichen nach folgendem Muster. Zum einen wurden in einer Minderheit der Verfahren die Fachbehörden bereits vor Abschluß der Vollständig-

366 Auf die fehlende Weisungsbefugnis der Genehmigungsbehörde gegenüber den Fachbehörden wurde bereits hingewiesen (Anm. 112).

367 Aus $§ 7$ Abs. 2 der 9. BImSchV läßt sich dagegen keine zeitliche Verpflichtung der Genehmigungsbehörde für die Fachbehördenbeteiligung ableiten, da diese Bestimmung ausschließlich Verpflichtungen gegenüber dem Antragsteller regelt. 
keitsprüfung zur Abgabe ihrer Stellungnahme aufgefordert. Beim GAA-N fällt dieser Anteil mit rd. zwei Zehnteln $(21,4 \%)$ annähernd doppelt so hoch aus wie beim LRA-1 mit rd. einem guten Zehntel $(11,6 \%) .{ }^{368}$ Zum anderen wurde in jeweils rd. der Hälfte der Verfahren beider Ämter $(50,0 \%$ bzw. $53,5 \%$ ) binnen einer Woche nach Feststellung der Unterlagenvollständigkeit und in einem weiteren Fünftel bzw. Viertel $(21,4 \%$ bzw. 25,6\%) binnen zweier Wochen mit der Behördenbeteiligung begonnen. In weniger als jeweils einem Zehntel der Fälle (7,1\% bzw. 9,3\%) erfolgte dieser Schritt erst später. ${ }^{369}$

Tab. 41.1

Dauer der Vorbereitung der Fachbehördenbeiziehung (Vollständigkeit der Unterlagen bis Beiziehung der ersten Fachbehörde) (Häufigkeitsverteilung)

\begin{tabular}{|l|c|c|c|}
\cline { 2 - 4 } \multicolumn{1}{c|}{} & GAA-N & LRA-1 & LRA-2 \\
\hline \multirow{2}{*}{ Beteiligung bereits eingeleitet } & 12 & 5 & 5 \\
& $21,4 \%$ & $11,6 \%$ & $20,8 \%$ \\
\hline \multirow{2}{*}{ Bis zu einer Woche } & 28 & 23 & 15 \\
& $50,0 \%$ & $53,5 \%$ & $62,5 \%$ \\
\hline \multirow{2}{*}{ Über eine bis zwei Wochen } & 12 & 11 & 1 \\
\hline \multirow{2}{*}{ Über zwei Wochen* } & $21,4 \%$ & $25,6 \%$ & $4,2 \%$ \\
\hline \hline \multirow{2}{*}{ Verfahren gesamt** } & 4 & 4 & 3 \\
& $7,1 \%$ & $9,3 \%$ & $12,5 \%$ \\
\hline
\end{tabular}

* Maximaler Wert beim GAA-N: 2,8 Mon., LRA-1: 2,2 Mon., LRA-2: 6,8 Mon.

** Abweichungen von $100,0 \%$ durch Rundungen bei den prozentualen Einzelhäufigkeiten.

${ }^{* * *}$ Die Gesamtzahl von 58 Verfahren verringert sich um zwei Fälle ohne Fachbehördenbeteiligung. ${ }^{370}$

Von einer bei beiden Genehmigungsbehörden insgesamt zügigen Eröffnung des Beteiligungsverfahrens zeugen auch die durchschnittlichen Dauern von

368 Die Differenz zwischen GAA-N und LRA-1 ist nicht signifikant. Das gilt auch für die Differenz zwischen LRA-1 und LRA-2.

369 Mehrheitlich gingen diese Fälle allerdings mit erheblicher Überziehung der ZweiWochen-Frist einher; vgl. dazu auch die in Tab. 41.1 ausgewiesenen Maximalwerte.

370 Vgl. oben Ziff. 2.3.1. mit Tab. 13.1. Die um zwei Fälle verminderte Gesamtheit der Verfahren beim GAA-N betrifft sämtliche nachfolgenden Tabellen zur Fachbehördenbeteiligung (bis einschl. Tab. 43.2). 
jeweils sieben Tagen ( $=0,23$ Mon. bei niedrigen Varianzen) sowie die noch darunter liegenden Mediane von 6,1 Tagen (=0,20 Mon.) beim GAA-N bzw. 4,9 Tagen (=0,16 Mon.) beim LRA-1 (Tab. 41.11)..$^{371}$

Tab. 41.11

Dauer der Vorbereitung der Fachbehördenbeiziehung (Vollständigkeit der Unterlagen bis Beiziehung der ersten Fachbehörde) (statistische Parameter in Monaten)*

\begin{tabular}{|l|c|c|c|}
\cline { 2 - 4 } \multicolumn{1}{c|}{} & GAA- $N$ & LRA-1 & LRA-2 \\
\hline Arith. Mittel & 0,23 & 0,23 & 0,20 \\
\hline Varianz & 0,06 & 0,08 & 0,05 \\
\hline Median & 0,20 & 0,16 & 0,16 \\
\hline Minimum & 0,00 & 0,00 & 0,00 \\
\hline Maximum & 1,11 & 1,44 & 0,89 \\
\hline \hline Gesamt & 42 & 36 & 17 \\
\hline
\end{tabular}

* Ohne Extremwerte und ohne die Verfahren, in denen bereits vor Feststellung der Vollständigkeit der Unterlagen die Behördenbeteiligung eingeleitet worden war (vgl. Zeile 1 in Tab. 41.1), beim GAA-N ferner abzüglich der zwei Fälle ohne Fachbehördenbeteiligung.

Das einheitliche Bild ändert sich, wenn man zusätzlich die Zeitspanne zwischen der als erste und der als letzte beteiligten Stelle berücksichtigt.

Wie Tab. 41.2 zeigt, lag zwischen der Aufforderung der ersten und letzten Fachbehörde zur Stellungnahme in $87,5 \%$ der Verfahren beim GAA-N weniger als ein Tag (erste Zeitkategorie). Dabei ist allerdings zu berücksichtigen, daß in etwas mehr als der Hälfte dieser Fälle ( 28 bzw. 57,1\% von 49 absolut, entsprechend 50,0\% der Grundgesamtheit von 56 absolut) für die Behördenbeteiligung nur ein einziger Adressat angesprochen wurde, nämlich die zentrale Dienststelle der Kommunal- bzw. Kreisverwaltungen. Dieser oblag es dann darüber zu entscheiden, welche der ihr zugehörigen Fachämter von der Anfrage tangiert sein könnten und demzufolge zu deren Bearbeitung aufzufordern wären. ${ }^{372}$ Lediglich in dem restlichen (kleineren) Teil der Zeitklasse von weniger als einem Tag ( 21 bzw. $42,9 \%$ von 49 absolut, entsprechend 37,5\% der Grundgesamtheit von 56 absolut) erstreckte

371 Eine Differenzierung nach Neu- und Änderungsgenehmigungen zeitigt dabei keine nennenswerten Unterschiede. Vgl. dazu Tab. 41.12-A im Anhang.

372 Vgl. oben Ziff. 2.3 mit Anm. 116 und Ziff. 2.3.2 mit Anm. 137. 
sich die Fachbehördenbeiziehung auf mindestens zwei (oder mehr) Adressaten. Nur in diesen Fällen läßt sich von einem »sternförmigen« Beteiligungsprozedere (i.S. des $\S 11$ Satz 2 der 9. BImSchV) sprechen (vgl. oben Ziff. 4.1).

Tab. 41.2

Dauer der Fachbehördenbeiziehung (Beiziehung der ersten bis Beiziehung der letzten Fachbehörde) (Häufigkeitsverteilung)

\begin{tabular}{|l|c|c|c|}
\cline { 2 - 4 } \multicolumn{1}{c|}{} & GAA-N & LRA-1 & LRA-2 \\
\hline Weniger als ein Tag & 49 & 19 & 6 \\
(bzw. "sternförmig") & $87,5 \%$ & $44,2 \%$ & $25,0 \%$ \\
\hline Bis zu zwei Wochen & 3 & 5 & 6 \\
& $5,4 \%$ & $11,6 \%$ & $25,0 \%$ \\
\hline Über zwei Wochen \\
bis zu einem Monat & 1 & 6 & 2 \\
\hline Über einen Monat* & $1,8 \%$ & $14,0 \%$ & $8,3 \%$ \\
\hline \hline Verfahren gesamt** & 3 & 13 & 10 \\
\hline \hline
\end{tabular}

* Maximaler Wert beim GAA-N: 8,2 Mon., LRA-1: 48,4 Mon., LRA-2: 8,3 Mon.

** Abweichungen von 100,0\% durch Rundungen bei den prozentualen Einzelhäufigkeiten.

${ }^{* * *}$ Um zwei Fälle ohne Fachbehördenbeteiligung verringerte Grundgesamtheit.

Beim LRA-1 entfallen dagegen mit einem Anteil von 44,2\% zwar nur rd. halb so viele Verfahren wie beim GAA-N in die erste Kategorie der Beiziehungsdauer. ${ }^{373}$ In diesen Fällen waren aber ausnahmslos mindestens zwei Adressaten einbezogen. Die hier - im Unterschied zu Niedersachsen - gegebene Dominanz dieser Konstellation ist im wesentlichen darauf zurückzuführen, daß das baden-württembergische Amt als Teil der Kreisverwaltung - in dieser Hinsicht der erwähnten »Zentrale« im Norden vergleichbar - selbst für die Beteiligung der im eigenen Haus angesiedelten Fachämter zu sorgen hat. Trägt man diesen unterschiedlichen (nur mittelbar modellbedingten) Randbedingungen Rechnung und konzentriert den Vergleich auf die Anteile (aus der Perspektive der Genehmigungsbehörde auch faktisch) »sternförmiger« Behördenbeiziehung, so schneidet das süddeutsche LRA1 etwas besser ab als das norddeutsche GAA-N (44,2\% gegenüber 37,5\%).

373 Die Differenz ist hochsignifikant $\left(\mathrm{chi}^{2}=21,24>10,82, \mathrm{p}=0,001, \mathrm{df}=1.\right)$. 
Wendet man sich den weiteren, längere Dauern repräsentierenden Zeitklassen $\mathrm{zu}$, verkehren sich die Relationen jedoch auffallend zu Lasten der baden-württembergischen Genehmigungsbehörde. Dort dauerte es in fast einem Drittel $(30,2 \%)$ aller Verfahren über einen Monat - überwiegend sogar mehrere Monate -, bis auch die letzte Fachbehörde einbezogen war. ${ }^{374}$ Solche Zeiten finden sich beim niedersächsischen Pendant eher nur in Ausnahmefällen $(5,4 \%) .{ }^{375}$ Der nicht nur vergleichsweise, sondern auch für sich genommen hohe Anteil in Baden-Württemberg bringt die durchschnittliche Dauer dieser Verfahrensphase, die in Niedersachsen unter einer Woche (5,5 Tage bzw. 0,18 Mon.) liegt, auf eine Höhe von 2,4 Monaten (Tab. 41.21). ${ }^{376}$ Dazu trugen vor allem die Änderungsgenehmigungen bei, die einen Durchschnittswert von 3,5 Monaten aufweisen gegenüber nur 0,74 Monaten (= rd. 3 Wochen) bei den Neugenehmigungen (Tab. 41.22). ${ }^{377}$ Eine ähnliche Tendenz zu längeren Dauern bei Änderungs- gegenüber Neugenehmigungen zeigt sich auch bei der niedersächsischen Genehmigungsbehörde. Die Differenz zwischen den beiden Genehmigungsarten $(0,25$ Mon. gegenüber 0,00 Mon.) ist hier aber auf Grund der weniger asymmetrischen Verteilung wesentlich schwächer ausgeprägt. ${ }^{378}$

374 Noch größer fällt der entsprechende Anteil mit 41,7\% beim LRA-2 aus.

375 Die Differenz ist hochsignifikant $\left(\mathrm{chi}^{2}=11,11>10,82, \mathrm{p}=0,001\right.$, $\mathrm{df}=1$. $)$.

376 Bei allerdings hoher Varianz (25,43 Mon.) wegen der asymmetrischen Verteilung mit den beiden Häufigkeitsschwerpunkten am unteren und oberen Ende. Trotz der ungleichen Varianzen (GAA-N: 0,64 Mon.) ergibt der statistische Test für die Mittelwertdifferenzen eine hohe Signifikanz $\left(t=3,156>t_{(0,999 ; 95)}=2,364\right.$ bei $F=39,61$ $\left.>\mathrm{F}_{(0,999 ; 41,54)}=2,31\right)$. - Auch beim LRA-2 liegt der Mittelwert mit 1,5 Mon. erheblich über dem beim GAA-N.

377 Trotz der auch hier signifikant differierenden Varianzen (39,30 Mon. gegenüber 1,53 Mon.: $\left.\mathrm{F}=25,74>\mathrm{F}_{(0,999 ; 24,16)}=4,99\right)$ divergieren die Mittelwerte (noch) signifikant $\left(\mathrm{t}=1,760>\mathrm{t}_{(0,95 ; 40)}=1,684\right)$.

378 Die Differenz der Mittelwerte bleibt unterhalb statistischer Signifikanz $(t=1,014$ $>\mathrm{t}_{(0,90 ; 53)}=1,299$ bei $\left.\mathrm{F}=0,00<\mathrm{F}_{(0,999 ; 39,14)}=2,27\right)$. - Der Durchschnittswert von 0,00 Mon. bei den Neugenehmigungen beim GAA-N bedeutet, daß hier die Fachbehördenbeiziehung ausnahmslos »sternförmig « erfolgte bzw. sich auf die kommunale »zentrale Dienststelle« beschränkte. 
Tab. 41.21

Dauer der Fachbehördenbeiziehung (Beiziehung der ersten bis Beiziehung der letzten Fachbehörde) (statistische Parameter in Monaten)*

\begin{tabular}{|l|c|c|c|}
\cline { 2 - 4 } \multicolumn{1}{c|}{} & GAA-N & LRA-1 & LRA-2 \\
\hline Arith. Mittel & 0,18 & 2,36 & 1,50 \\
\hline Varianz & 0,64 & 25,43 & 3,88 \\
\hline Median & 0,00 & 0,17 & 0,46 \\
\hline Minimum & 0,00 & 0,00 & 0,00 \\
\hline Maximum & 4,82 & 21,54 & 7,51 \\
\hline \hline Gesamt** & 55 & 42 & 23 \\
\hline
\end{tabular}

* Ohne Extremwerte, beim GAA-N ferner abzüglich der zwei Fälle ohne Fachbehördenbeteiligung.

Tab. 41.22

Dauer der Fachbehördenbeiziehung (Beiziehung der ersten bis Beiziehung der letzten Fachbehörde), getrennt nach Neu- und Änderungsgenehmigungen (statistische Parameter in Monaten) ${ }^{*}$

\begin{tabular}{|l|c|c|c|c|c|c|}
\cline { 2 - 8 } & \multicolumn{2}{c|}{$G A A-N$} & \multicolumn{2}{c|}{$L R A-1$} & \multicolumn{2}{c|}{ LRA-2 } \\
& Änd.-G & Neu-G & Änd.-G & Neu-G & Änd.-G & Neu-G \\
\hline Arith. Mittel & $\mathbf{0 , 2 5}$ & 0,00 & $\mathbf{3 , 4 6}$ & 0,74 & $\mathbf{1 , 3 5}$ & 1,67 \\
\hline Varianz & $\mathbf{0 , 8 7}$ & 0,00 & $\mathbf{3 9 , 3 0}$ & 1,53 & $\mathbf{5 , 1 4}$ & 2,83 \\
\hline Median & $\mathbf{0 , 0 0}$ & 0,00 & $\mathbf{0 , 2 3}$ & 0,07 & $\mathbf{0 , 2 8}$ & 0,66 \\
\hline Minimum & $\mathbf{0 , 0 0}$ & 0,00 & $\mathbf{0 , 0 0}$ & 0,00 & $\mathbf{0 , 0 0}$ & 0,00 \\
\hline Maximum & $\mathbf{4 , 8 2}$ & 0,00 & $\mathbf{2 1 , 5 4}$ & 4,36 & $\mathbf{7 , 5 1}$ & 4,43 \\
\hline \hline Gesamt & $\mathbf{4 0}$ & 15 & $\mathbf{2 5}$ & 17 & $\mathbf{1 2}$ & 11 \\
\hline
\end{tabular}

* Ohne Extremwerte, beim GAA-N ferner abzüglich der zwei Fälle ohne Fachbehördenbeteiligung.

Aus den dargestellten Befunden geht klar hervor, daß die Phase der Fachbehördenbeiziehung der norddeutschen Genehmigungsbehörde ausweis- 
lich ihres erheblich geringeren durchschnittlichen Zeitverbrauchs offenkundig weniger Probleme bereitete als der süddeutschen. Dafür ließe sich der im niedersächsischen Modell mittelbar gegebene Vorteil verantwortlich machen, daß hier für eine Reihe zu beteiligender Fachämter eine zentrale Dienststelle als Adressat zur Verfügung steht, an die andernfalls selbst zu erbringende Koordinationsleistungen delegiert werden können. Die höhere Koordinationslast im Süden vermag allerdings nicht den Befund zu erklären, daß die baden-württembergische Genehmigungsbehörde in erster Linie bei Änderungsgenehmigungen vergleichsweise viel Zeit für die Behördenbeiziehung in Anspruch nahm, indem sie in diesen Verfahren die Entscheidung über die zu beteiligenden Fachämter nicht zeitlich gebündelt, sondern nacheinander nach Maßgabe des weiteren Verfahrensverlaufs traf. Es ist nicht auszuschließen, daß sich dieses sukzessive Vorgehen auch einer gewissen Unsicherheit bei der genehmigungsrechtlichen Prüfung von Änderungen bereits bestehender Anlagen verdankt.

Der Norden hat mit seiner eher zügig betriebenen Beiziehung der Fachbehörden einerseits eine wesentliche Voraussetzung dafür geschaffen, daß auch die gesamte Phase des fachbehördlichen Beteiligungsverfahrens - hier definiert als Zeitspanne zwischen der zeitlich zuerst ergangenen Anforderung einer Stellungnahme und der zeitlich zuletzt eingegangenen Stellungnahme - kürzer ausfälit als im Süden. Andererseits unterliegt die niedersächsische Genehmigungsbehörde jedoch dem Risiko, den zeitlichen »Vorsprung « dadurch zu verlieren, daß die zentrale Dienststelle die an sie delegierte Beiziehung der in ihrem Haus betroffenen Fachämter nur mit zeitlich nachgeordneter Priorität behandelt. Demgegenüber könnte die selbst als »Zentrale« fungierende baden-württembergische Genehmigungsbehörde den durch den höheren Koordinationsbedarf bedingten zusätzlichen Zeitaufwand bei der Behördenbeiziehung durch das Privileg »wettmachen«, über den internen »kleinen « Dienstweg auf die hauseigenen Ämter zugunsten einer zügigen Erarbeitung der Stellungnahmen einzuwirken. Der nachfolgende Abschnitt wird allerdings zeigen, daß der Norden den in der Beiziehungsphase (mittelbar modellbedingt) erzielten Zeitgewinn in der Phase der Fachbehördenbeteiligung weitgehend bewahren und der Süden seinen anfänglichen Zeitverlust kaum (durch mittelbar modellbedingte Vorteile) kompensieren kann.

\subsubsection{Fachbehördliche Anfertigung der Stellungnahmen}

Als (Gesamt-) Dauer der fachbehördlichen Mitwirkung am Genehmigungsverfahren durch Anfertigen vorhabenbezogener Begutachtungen ist hier wie erwähnt - die zwischen der zeitlich ersten Anforderung einer Stellungnahme und der zeitlich zuletzt eingegangenen Stellungnahme vergangene 
Zeitspanne definiert. Tab. 42.1 und Tab. 42.11 geben die entsprechenden Befunde (Häufigkeitsverteilung nach Zeitklassen bzw. zugehörige statistische Parameter) wieder.

Die größte Divergenz in den Häufigkeitsverteilungen in Tab. 42.1 tritt in der Kategorie der Dauer von mehr als einem bis höchstens zwei Monaten auf. In ihr sind beim GAA-N mehr als ein Viertel $(28,6 \%)$, beim LRA-1 dagegen weniger als ein Zehntel $(9,3 \%)$ der Verfahren versammelt. Nimmt man noch die jeweils wenigen Verfahren hinzu, die in die unterste Kategorie einer Dauer von maximal einem Monat fallen, ist zugleich der Hauptunterschied zwischen den beiden Ämtern markiert. Bei der niedersächsischen Genehmigungsbehörde war demnach die gesamte Phase der fachbehördlichen Verfahrensbeteiligung zu einem guten Drittel $(35,7 \%)$, bei der baden-württembergischen aber nur - statistisch signifikant abweichend - 379 zu einem Siebtel $(14,0 \%)$ der Verfahren binnen zweier Monate abgeschlossen (Kumulation der ersten beiden Kategorien der Tabelle). ${ }^{380}$ In der Tendenz spiegelbildlich dazu beläuft sich der Anteil der Verfahren mit einer längeren Beteiligungsdauer von mehr als zwei bis höchstens sechs Monaten beim GAA-N lediglich auf 37,5\%, beim LRA-1 auf 51,2\% (Kumulation der dritten und vierten Kategorie der Tabelle). Bei den Verfahren mit einer besonders langwierigen Beteiligungsphase von über sechs bis höchstens 12 Monaten bzw. über 12 Monaten (die letzten beiden Kategorien der Tabelle) konvergieren die beiden Ämter am stärksten bei vergleichsweise geringer ausgeprägten Differenzen. Die Konvergenz spielt sich freilich auf relativ hohem Niveau ab mit Anteilen von 26,8\% (GAA-N) und 34,9\% (LRA-1) an Fällen, die für diese Phase mehr als sechs Monate, mithin mehr als doppelt so viel Zeit, wie gesetzlich für die gesamte Dauer des Genehmigungsverfahrens zugestanden, benötigten (Kumulation der letzten beiden Kategorien der Tabelle). Dabei steuern Verfahren mit Beteiligungszeiten von über 12 Monaten beim LRA-1 ein (relativ) größeres Kontingent bei als beim GAA-N. ${ }^{381}$

$379 \mathrm{chi}^{2}=5,94>5,02, \mathrm{p}=0,025, \mathrm{df}=1$.

380 Der entsprechende Wert beim LRA-2 von 33,3\% entspricht allerdings nahezu dem beim GAA-N.

381 Die Differenz der Anteile von 18,6\% und 8,6\% beim LRA-1 bzw. GAA-N in dieser Kategorie ist aber nicht signifikant $\left(\mathrm{chi}^{2}=2,53<2,71\right.$ für $\mathrm{p}=0,1$ und $\left.\mathrm{df}=1\right)$. 
Tab. 42.1

Gesamtdauer der Fachbehördenbeteiligung (erste angeforderte Stellungnahme bis Eingang der letzten Stellungnahme) (Häufigkeitsverteilung)

\begin{tabular}{|c|c|c|c|}
\hline & \\
\hline & $G A A-N$ & LRA-1 & $L R A-2$ \\
\hline Bis zu einem Monat & $\begin{array}{c}4 \\
7,1 \%\end{array}$ & $\begin{array}{c}2 \\
4,7 \%\end{array}$ & $\begin{array}{c}1 \\
4,2 \%\end{array}$ \\
\hline Über einen bis zwei Monate & $\begin{array}{c}16 \\
28,6 \%\end{array}$ & $\begin{array}{c}4 \\
9,3 \%\end{array}$ & $\begin{array}{c}7 \\
29,2 \%\end{array}$ \\
\hline Über zwei bis drei Monate & $\begin{array}{c}14 \\
25,0 \%\end{array}$ & $\begin{array}{c}9 \\
20,9 \%\end{array}$ & $\begin{array}{c}2 \\
8,3 \%\end{array}$ \\
\hline Über drei bis sechs Monate & $\begin{array}{c}7 \\
12,5 \%\end{array}$ & $\begin{array}{c}13 \\
30,2 \%\end{array}$ & $\begin{array}{c}9 \\
37,5 \%\end{array}$ \\
\hline Über sechs bis zwölf Monate & $\begin{array}{c}10 \\
17,9 \%\end{array}$ & $\begin{array}{c}7 \\
16,3 \%\end{array}$ & $\begin{array}{c}4 \\
16,7 \%\end{array}$ \\
\hline Über zwölf Monate* & $\begin{array}{c}5 \\
8,9 \%\end{array}$ & $\begin{array}{c}8 \\
18,6 \%\end{array}$ & $\begin{array}{c}1 \\
4,2 \%\end{array}$ \\
\hline Verfahren gesamt** & $\begin{array}{l}56 * * * \\
100,0 \%\end{array}$ & $\begin{array}{c}43 \\
100,0 \%\end{array}$ & $\begin{array}{c}19 \\
100,1 \%\end{array}$ \\
\hline
\end{tabular}

* Maximaler Wert beim GAA-N: 33,9 Mon., LRA-1: 48,8 Mon., LRA-2: 13,7 Mon.

** Abweichungen von $100,0 \%$ durch Rundungen bei den prozentualen Einzelhäufigkeiten.

*** Um zwei Fälle ohne Fachbehördenbeteiligung verringerte Grundgesamtheit.

Über der Sechsmonatsgrenze liegt auch die durchschnittliche Dauer der baden-württembergischen Fachbehördenbeteiligung mit 6,4 Monaten, während die niedersächsische Dauer mit 4,4 Monaten deutlich darunter liegt (Tab. 42.11) ${ }^{382}$ Selbst der Median, auf den weniger als auf den Mittelwert besonders lange Einzeldauern »durchschlagen «, überschreitet beim LRA-1 mit 4,0 Monaten die Dreimonatsgrenze, die beim GAA-N dagegen mit 2,4 Monaten ohne Mühe unterboten wird.

382 Die Differenz ist signifikant $\left(\mathrm{t}=1,990>\mathrm{t}_{(0,975 ; 95)}=1,984\right.$ bei $\mathrm{F}=2,14<\mathrm{F}_{(0,999 ; 41}$, $54)=2,31$ ) 
Tab. 42.11

Gesamtdauer der Fachbehördenbeteiligung (erste angeforderte Stellungnahme bis Eingang der letzten Stellungnahme) (statistische Parameter in Monaten) ${ }^{*}$

\begin{tabular}{|l|c|c|c|}
\cline { 2 - 4 } \multicolumn{1}{c|}{} & GAA-N & LRA-1 & LRA-2 \\
\hline Arith. Mittel & 4,35 & 6,42 & 3,90 \\
\hline Varianz & 17,11 & 36,86 & 6,63 \\
\hline Median & 2,36 & 3,99 & 3,96 \\
\hline Minimum & 0,79 & 0,30 & 0,89 \\
\hline Maximum & 19,08 & 23,34 & 9,28 \\
\hline \hline Gesamt & 55 & 42 & 23 \\
\hline
\end{tabular}

* Ohne Extremwerte, beim GAA-N ferner abzüglich der zwei Fälle ohne Fachbehördenbeteiligung.

Zum erhöhten Zeitbedarf für die Fachbehördenbeteiligung haben beim LRA-1 - wie schon in der Beiziehungsphase (Ziff. 4.3.3.1) - in erster Linie die Änderungsgenehmigungen beigetragen (Tab. 42.12). Sie übertreffen mit einem Durchschnittswert von 7,4 Monaten deutlich den bei Neugenehmigungen von 5,0 Monaten. ${ }^{383}$ Beim GAA-N bewegt sich der entsprechende Wert für Neugenehmigungen mit 5,1 Monaten auf etwa gleicher Höhe, während dort der Durchschnittswert für Änderungsgenehmigungen mit 4,1 Monaten signifikant unter dem entsprechenden Wert beim LRA-1 liegt. ${ }^{384}$

383 Die Differenz ist aber nicht signifikant $\left(t=1,265<\mathrm{t}_{(0,90 ; 40)}=1,303\right.$ bei $\mathrm{F}=2,11<$ $\left.\mathrm{F}_{(0,95 ; 24,16)}=2,24\right)$.

$384 \mathrm{t}=2,438>\mathrm{t}_{(0,99 ; 63)}=2,390$ bei $\left.\mathrm{F}=2,61<\mathrm{F}_{(0,999 ; 24,39)}=2,87\right)$. Beim GAA-N ist dagegen die Differenz der Mittelwerte zwischen Neu- und Änderungsgenehmigungen nicht signifikant $\left(\mathrm{t}=0,817<\mathrm{t}_{(0,90 ; 53)}=1,299\right.$ bei $\left.\mathrm{F}=1,10<\mathrm{F}_{(0,95 ; 39,14)}=2,20\right)$. 
Tab. 42.12

Gesamtdauer der Fachbehördenbeteiligung (erste angeforderte Stellungnahme bis Eingang der letzten Stellungnahme), getrennt nach Neu- und Änderungsgenehmigungen (statistische Parameter in Monaten)*

\begin{tabular}{|c|c|c|c|c|c|c|}
\hline & \multicolumn{2}{|c|}{$G A A-N$} & \multicolumn{2}{|c|}{$L R A-1$} & \multicolumn{2}{|c|}{$L R A-2$} \\
\hline & Änd.-G & $\mathrm{Neu}-\mathrm{G}$ & Änd.-G & Neu-G & Änd.-G & Neu-G \\
\hline Arith. Mittel & 4,07 & 5,10 & 7,39 & 4,99 & 3,49 & 4,27 \\
\hline Varianz & 17,63 & 16,05 & 46,03 & 21,77 & 7,73 & 5,91 \\
\hline Median & 2,15 & 3,70 & 4,33 & 3,41 & 2,36 & 4,19 \\
\hline Minimum & $\mathbf{0 , 8 2}$ & 0,79 & $\mathbf{0 , 3 0}$ & 0,92 & 0,89 & 1,38 \\
\hline Maximum & 19,08 & 13,64 & 23,34 & 20,72 & 9,28 & 8,85 \\
\hline Gesamt & 40 & 15 & 25 & 17 & 11 & 12 \\
\hline
\end{tabular}

* Ohne Extremwerte, beim GAA-N ferner abzüglich der zwei Fälle ohne Fachbehördenbeteiligung.

Als Zwischenresümee läßt sich festhalten, daß die im Süden gegenüber dem Norden erheblich größere zeitliche Streckung der fachbehördlichen Beiziehungsphase (Ziff. 4.3.3.1) im großen und ganzen ungebrochen auf die Dauer der (gesamten) Phase der fachbehördlichen Mitwirkung vom Beginn der Anforderung der Stellungnahmen bis zum Eingang der letzten Stellungnahme bei der Genehmigungsbehörde »durchschlägt«. Dieses Ergebnis deutet darauf hin, daß sich die Fachbehörden im Hinblick auf die Zeit, die sie durchschnittlich für die Erarbeitung ihrer Stellungnahmen benötigten, in den beiden Modellen nicht wesentlich unterscheiden. Daß dem tatsächlich so ist, läßt sich an der Dauer von der Beiziehung der letzten Fachbehörde bis zum Eingang der letzten fachbehördlichen Stellungnahme demonstrieren. $\mathrm{Ob}$ es sich dabei um dieselbe oder verschiedene Behörden handelt, ist im vorliegenden Zusammenhang ohne Belang.

Tab. 42.2 zeigt die Häufigkeitsverteilung der entsprechenden Dauern auf verschiedene Zeitklassen. Sie fällt beim GAA-N und beim LRA-1 weitgehend ähnlich aus mit jeweils geringeren Häufigkeiten in der untersten (weniger als ein Monat) und obersten (über 12 Monate) Zeitklasse und den gröBeren Häufigkeiten in den mittleren Zeitklassen. ${ }^{385}$

385 Die größte Differenz mit 8,4 Prozentpunkten (in der vierten Zeitklasse) ist statistisch nicht signifikant $\left(\mathrm{chi}^{2}=1,28<2,71\right.$ für $\mathrm{p}=0,1$ und $\left.\mathrm{df}=1\right)$. 
Tab. 42.2

(Mindest-) Dauer der fachbehördlichen Erarbeitung der Stellungnahmen (zuletzt beigezogene Fachbehörde bis Eingang der letzten fachbehördlichen Stellungnahme) (Häufigkeitsverteilung)

\begin{tabular}{|l|c|c|c|}
\cline { 2 - 4 } \multicolumn{1}{c|}{} & GAA-N & $L R A-1$ & $L R A-2$ \\
\hline Bis zu einem Monat & 5 & 6 & 8 \\
\hline Über einen bis zwei Monate & $8,9 \%$ & $14,0 \%$ & $33,3 \%$ \\
\hline Über zwei bis drei Monate & 15 & 9 & 6 \\
& $26,8 \%$ & $20,9 \%$ & $25,0 \%$ \\
\hline Über drei bis sechs Monate & 16 & 10 & 3 \\
\hline Über sechs bis zwölf Monate & $28,6 \%$ & $23,3 \%$ & $12,5 \%$ \\
\hline Über zwölf Monate* & 7 & 9 & 5 \\
\hline \hline Verfahren gesamt** & $12,5 \%$ & $20,9 \%$ & $20,8 \%$ \\
\hline \hline
\end{tabular}

* Maximaler Wert beim GAA-N: 33,9 Mon., LRA-1: 17,5 Mon., LRA-2: 8,7 Mon.

** Abweichungen von 100,0\% durch Rundungen bei den prozentualen Einzelhäufigkeiten.

${ }^{* * *}$ Um zwei Fälle ohne Fachbehördenbeteiligung verringerte Grundgesamtheit.

Im Norden wie im Süden beläuft sich die durchschnittliche Zeitspanne zwischen der zuletzt ergangenen Aufforderung an eine Fachbehörde zur Äußerung und der zuletzt bei der Genehmigungsbehörde eingegangenen Stellungnahme auf jeweils über dreieinhalb Monate (Tab. 42.21). Mit dieser Dauer überschreiten beide Ämter die gesetzlich für die gesamte Fachbehördenbeteiligung vorgesehene Monatsfrist um mehr als das Dreifache, wobei die baden-württembergische Behörde mit 3,65 Monaten etwas günstiger (aber nicht signifikant) abschneidet als die niedersächsische mit 3,95 Monaten. ${ }^{386}$ Auch zwischen Neu- und Änderungsgenehmigungen weichen die Mittelwerte weder im intra- noch im interbehördlichen Vergleich in statistisch erheblichem Ausmaß voneinander ab (Tab. 42.22). ${ }^{387}$

$386 \mathrm{t}=0,396<\mathrm{t}_{(0,90 ; 95)}=1,290$ bei $\mathrm{F}=1,49<\mathrm{F}_{(0,95 ; 54,41)}=1,61$.

387 Selbst für die größte Differenz zwischen den Mittelwerten bei den Neugenehmigungen beim GAA-N bzw. LRA-1 (4,55 Mon. bzw. 3,42 Mon.), ergibt sich kein signifikanter Testwert $\left(t=1,148<\mathrm{t}_{(0,90 ; 29)}=1,310\right.$ bei $\left.\mathrm{F}=2,27<\mathrm{F}_{(0,95 ; 14,15)}=2,42\right)$. 
Tab. 42.21

(Mindest-) Dauer der fachbehördlichen Erarbeitung der Stellungnahmen (zuletzt beigezogene Fachbehörde bis Eingang der letzten fachbehördlichen Stellungnahme) (statistische Parameter in Monaten)*

\begin{tabular}{|l|c|c|c|}
\cline { 2 - 4 } \multicolumn{1}{c|}{} & GAA-N & LRA-1 & LRA-2 \\
\hline Arith. Mittel & 3,95 & 3,65 & 2,26 \\
\hline Varianz & 15,47 & 10,39 & 3,67 \\
\hline Median & 2,26 & 2,61 & 1,64 \\
\hline Minimum & 0,46 & 0,30 & 0,13 \\
\hline Maximum & 19,08 & 14,82 & 6,23 \\
\hline \hline Gesamt & 55 & 42 & 23 \\
\hline
\end{tabular}

* Ohne Extremwerte, beim GAA-N ferner abzüglich der zwei Fälle ohne Fachbehördenbeteiligung.

Tab. 42.22

(Mindest-) Dauer der fachbehördlichen Erarbeitung der Stellungnahmen (zuletzt beigezogene Fachbehörde bis Eingang der letzten fachbehördlichen Stellungnahme), getrennt nach Neu- und Änderungsgenehmigungen (statistische Parameter in Monaten) ${ }^{*}$

\begin{tabular}{|l|r|r|r|r|r|r|}
\cline { 2 - 8 } \multicolumn{1}{c|}{} & \multicolumn{2}{c|}{$G A A-N$} & \multicolumn{2}{c|}{ LRA-1 } & \multicolumn{2}{c|}{ LRA-2 } \\
\cline { 2 - 8 } & Änd.-G & Neu-G & Änd.-G & Neu-G & Änd.-G & Neu-G \\
\hline Arith. Mittel & $\mathbf{3 , 7 2}$ & 4,55 & $\mathbf{3 , 7 9}$ & 3,42 & $\mathbf{2 , 4 8}$ & 2,05 \\
\hline Varianz & $\mathbf{1 7 , 4 6}$ & 10,52 & $\mathbf{1 4 , 2 1}$ & 4,63 & $\mathbf{3 , 8 1}$ & 3,79 \\
\hline Median & $\mathbf{2 , 1 5}$ & 3,70 & $\mathbf{2 , 3 2}$ & 2,77 & $\mathbf{1 , 8 7}$ & 1,13 \\
\hline Minimum & $\mathbf{0 , 4 6}$ & 0,79 & $\mathbf{0 , 3 0}$ & 0,92 & $\mathbf{0 , 1 6}$ & $\mathbf{0 , 1 3}$ \\
\hline Maximum & $\mathbf{1 9 , 0 8}$ & 11,87 & $\mathbf{1 4 , 8 2}$ & 8,07 & $\mathbf{6 , 2 3}$ & 5,70 \\
\hline \hline Gesamt & $\mathbf{4 0}$ & 15 & $\mathbf{2 6}$ & 16 & $\mathbf{1 1}$ & 12 \\
\hline
\end{tabular}

* Ohne Extremwerte, beim GAA-N ferner abzüglich der zwei Fälle ohne Fachbehördenbeteiligung. 
Die dargestellten Befunde zur zeitlichen Belastung der Genehmigungsverfahren durch Beteiligung der Fachbehörden am Verfahren einschließlich ihrer Beiziehung lassen sich unter dem Gesichtspunkt des Modellvergleichs zu folgendem abschließenden Ergebnis resümieren. Im Hinblick auf die von den Genehmigungsbehörden im Norden wie im Süden kaum beeinflußbare Dauer der fachbehördlichen Erarbeitung der Stellungnahmen gleichen sich die Modelle. Sie unterscheiden sich dagegen im Hinblick auf die jeweils in der Hand der Genehmigungsbehörde selbst liegenden Beiziehungsdauer erheblich. Eindeutige Anhaltspunkte für modellbedingte Ursachen dieser Divergenz lassen sich den Befunden nicht entnehmen. Von daher kommen für die Verzögerungen der Behördenbeiziehung bei der süddeutschen Genehmigungsbehörde auch »hausgemachte (modellunabhängige) Defizite in der Selbstorganisation und in der Steuerung der behördeninternen Koordination und Kooperation in Frage. ${ }^{388}$

\subsubsection{Erstellung des Genehmigungsbescheids}

Die sich an die Fachbehördenbeteiligung anschließende, letzte Phase des Verfahrens ist die Erstellung des (offiziellen, rechtsverbindlichen) Genehmigungsbescheids.

\subsubsection{Erarbeitung der Endfassung}

Tab. 43 stellt die Zeiten (klassifiziert) dar, die die Genehmigungsbehörden nach Eingang der letzten fachbehördlichen Stellungnahme für die Erarbeitung der Endfassung bzw. den Erlaß des abschließenden Genehmigungsbescheids benötigten.

Aus ihr ist der wesentliche Unterschied der Verteilungen zwischen GAA-N und LRA-1 leicht ersichtlich. Während bei der niedersächsischen Behörde die größeren Anteile jeweils auf die drei die längeren Dauern markierenden Kategorien entfallen, verhält es sich beim LRA-1, wo die drei für die kürzeren Dauern stehenden Kategorien stärker besetzt sind, umgekehrt (in der Tabelle durch verstärkte Umrahmung hervorgehoben). Kumuliert man jeweils die Anteile der besagten Kategorien, ergibt sich, daß beim LRA-1 rd. drei Viertel (74,4\%) der Genehmigungsbescheide innerhalb von vier Wochen erstellt wurden und entsprechend nur ein Viertel $(25,6 \%) \mathrm{mehr}$ als einen Monat beanspruchte. ${ }^{389}$ Dagegen wurde beim GAA-N nicht ein-

388 Dose 1994, S. 236.

389 Beim LRA-2 bewegen sich die entsprechenden Anteile mit 66,7\% bzw. 33,3\% auf vergleichbarem Niveau. 
mal die Hälfte $(41,1 \%)$ der Bescheide binnen Monatsfrist verfaßt und entsprechend in über der Hälfte $(58,9 \%)$ der Fälle diese Frist überschritten. ${ }^{390}$

\section{Tab. 43}

Dauer der Erarbeitung des Genehmigungsbescheids (zuletzt eingegangene fachbehördliche Stellungnahme bis Ausfertigung des endgültigen Bescheids) (Häufigkeitsverteilung)

\begin{tabular}{|l|c|c|c|}
\cline { 2 - 4 } \multicolumn{1}{c|}{} & $G A A-N$ & $L R A-1$ & $L R A-2$ \\
\hline \multirow{2}{*}{ Bis zu einer Woche } & 5 & 14 & 3 \\
& $8,9 \%$ & $32,6 \%$ & $12,5 \%$ \\
\hline Über eine bis zwei Wochen & 10 & 7 & 8 \\
& $17,9 \%$ & $16,3 \%$ & $33,3 \%$ \\
\hline Über zwei bis vier Wochen & 8 & 11 & 5 \\
\hline \multirow{2}{*}{ Über einen bis zwei Monate } & $14,3 \%$ & $25,6 \%$ & $20,8 \%$ \\
\hline \multirow{2}{*}{ Über zwei bis drei Monate } & 15 & 5 & 2 \\
\hline Über drei Monate* & $26,8 \%$ & $11,6 \%$ & $8,3 \%$ \\
\hline \hline Verfahren gesamt** & 9 & 3 & 0 \\
& $16,1 \%$ & $7,0 \%$ & $0,0 \%$ \\
\hline
\end{tabular}

* Maximaler Wert beim GAA-N: 12,5 Mon., LRA-1: 8,8 Mon., LRA-2: 7,3 Mon.

** Abweichungen von 100,0\% durch Rundungen bei den prozentualen Einzelhäufigkeiten.

${ }^{* * *}$ Um zwei Fälle ohne Fachbehördenbeteiligung verringerte Grundgesamtheit.

Die fast spiegelbildliche Gegenläufigkeit der Verteilungen hat erheblich divergierende Durchschnittswerte für die Dauer dieser letzten Verfahrensphase zur Folge (Tab. 43.1), welche beim niedersächsischen Amt mit 1,7 Monaten (= gut 7 Wochen) fast doppelt so lang ausfällt wie beim badenwürttembergischen mit 0,9 Monaten (= knapp 4 Wochen) ${ }^{391}$ Die Unterschiede der Mittelwerte zwischen den beiden Ämtern ändern sich nicht wesentlich, wenn man Neu- und Änderungsgenehmigungen gesondert betrachtet. Das heißt andererseits, daß jeweils behördenintern zwischen den

390 Die Differenz ist hochsignifikant $\left(\mathrm{chi}^{2}=10,87>10,82, \mathrm{p}=0,001, \mathrm{df}=1\right)$.

391 Auch diese Differenz ist hochsignifikant $\left(t=2,886>t_{(0,995 ; 95)}=2,639\right.$ bei $F=1,87$ $\left.<\mathrm{F}_{(0,99 ; 54,41)}=2,02\right)$. 
Verfahrensarten keine größeren Differenzen in der durchschnittlichen Dauer bestehen (Tab. 43.2). ${ }^{392}$

\section{Tab. 43.1}

Dauer der Erarbeitung des Genehmigungsbescheids (zuletzt eingegangene fachbehördliche Stellungnahme bis Ausfertigung des endgültigen Bescheids) (statistische Parameter in Monaten)*

\begin{tabular}{|l|c|c|c|}
\cline { 2 - 4 } \multicolumn{1}{c|}{} & GAA-N & LRA-1 & LRA-2 \\
\hline Arith. Mittel & 1,68 & 0,86 & 1,43 \\
\hline Varianz & 2,36 & 1,26 & 3,42 \\
\hline Median & 1,38 & 0,51 & 0,52 \\
\hline Minimum & 0,00 & $-0,03^{* *}$ & 0,03 \\
\hline Maximum & 7,15 & 5,61 & 6,20 \\
\hline \hline Gesamt & 55 & 42 & 23 \\
\hline
\end{tabular}

* Ohne Extremwerte, beim GAA-N ferner abzüglich der zwei Fälle ohne Fachbehördenbeteiligung.

** $-0,03=1$ Tag, betrifft den einzigen Fall, bei dem 1 Tag vor Zusendung der fachbehördlichen Stellungnahme eine telephonische Vorabübermittlung erfolgte. Dies gilt auch für die Tab.

4.3.2.

Eine maßgebliche Ursache für die nahezu doppelte Zeit, die das GAA-N gegenüber dem LRA-1 für die Erstellung des Genehmigungsbescheids nach Eingang der letzten fachbehördlichen Stellungnahme gebraucht hat, ist leicht auszumachen. Sie liegt in der erwähnten, beim niedersächsischen Amt verbreiteten Praxis, dem Antragsteller zuvor eine Entwurfsfassung zu überstellen und ihm Gelegenheit zur Gegenäußerung zu geben (vgl. oben Ziff. 3.2).

392 Auch für die größere (behördeninterne) Differenz zwischen den durchschnittlichen Dauern der Neu- und Änderungsgenehmigungen beim LRA-1 (0,65 Mon. bzw. 1,01 Mon.), ergibt sich ein eindeutig nicht signifikanter Testwert von $\mathrm{t}=1,031<\mathrm{t}_{(0,90}$; ${ }_{40)}=1,303$ bei $\mathrm{F}=3,04<\mathrm{F}_{(0,99 ; 24,16)}=3,18$. 
Tab. 43.2

Dauer der Erarbeitung des Genehmigungsbescheids (zuletzt eingegangene fachbehördliche Stellungnahme bis Ausfertigung des endgültigen $\mathrm{Be}-$ scheids), getrennt nach Neu- und Änderungsgenehmigungen (statistische Parameter in Monaten)*

\begin{tabular}{|l|c|c|c|c|c|c|}
\cline { 2 - 8 } \multicolumn{1}{c|}{} & \multicolumn{2}{c|}{$G A A-N$} & \multicolumn{2}{c|}{$L R A-1$} & \multicolumn{2}{c|}{ LRA-2 } \\
\cline { 2 - 8 } & Änd.-G & Neu-G & Änd.-G & Neu-G & Änd.-G & Neu-G \\
\hline Arith. Mittel & $\mathbf{1 , 7 0}$ & 1,63 & $\mathbf{1 , 0 1}$ & 0,65 & $\mathbf{2 , 1 6}$ & 0,76 \\
\hline Varianz & $\mathbf{2 , 9 9}$ & 0,91 & $\mathbf{1 , 7 2}$ & 0,57 & $\mathbf{4 , 9 6}$ & 1,30 \\
\hline Median & $\mathbf{1 , 0 8}$ & 1,77 & $\mathbf{0 , 5 2}$ & 0,39 & $\mathbf{1 , 2 8}$ & 0,43 \\
\hline Minimum & $\mathbf{0 , 0 0}$ & 0,13 & $\mathbf{0 , 0 7}$ & $-0,03$ & $\mathbf{0 , 1 3}$ & 0,03 \\
\hline Maximum & $\mathbf{7 , 1 5}$ & 3,15 & $\mathbf{5 , 6 1}$ & 2,95 & $\mathbf{6 , 2 0}$ & 4,30 \\
\hline \hline Gesamt & $\mathbf{3 9}$ & 16 & $\mathbf{2 5}$ & 17 & $\mathbf{1 1}$ & 12 \\
\hline
\end{tabular}

* Ohne Extremwerte, beim GAA-N ferner abzüglich der zwei Fälle ohne Fachbehördenbeteiligung.

\subsubsection{Revision der Entwurfsfassung beim GAA-N}

Tab. 44 gibt Auskunft über die Dauer, die beim GAA-N zwischen Ausfertigung der Entwurfsfassung und der endgültigen Fassung des Genehmigungsbescheids vergangen ist. 
Tab. 44

Dauer der Revisionsphase für die Entwurfsfassung des Genehmigungsbescheids (Ausfertigung der Entwurfsfassung bis Ausfertigung des endgültigen Bescheids) (oben Häufigkeitsverteilung, unten statistische Parameter in Monaten) - Nur GAA-N - ${ }^{*}$

\begin{tabular}{|l|c|}
\cline { 2 - 2 } \multicolumn{1}{c|}{} & Häufigkeiten \\
\hline \multirow{2}{*}{ Bis zu zwei Wochen } & 5 \\
& $17,9 \%$ \\
\hline Über zwei bis vier Wochen & 10 \\
Über einen bis drei Monate & $35,7 \%$ \\
\hline \multirow{2}{*}{ Über drei Monate** } & 9 \\
\hline \hline \multirow{2}{*}{ Verfahren mit Entwurf gesamt*** } & $42,1 \%$ \\
\hline
\end{tabular}

Beim LRA-1 betragen die Zeiten für die beiden (»unfreiwilligen «) ${ }^{393}$ einschlägigen Fälle 1,15 bzw. 5,77 Mon.

** Maximaler Wert: 10,0 Mon.

*** Ausgenommen der Entwurf eines Teilgenehmigungsbescheids (s. Anm. 393).

\begin{tabular}{|l|c|}
\cline { 2 - 2 } \multicolumn{1}{c|}{} & Parameter $^{*}$ \\
\hline Arith. Mittel & 1,44 \\
\hline Varianz & 1,96 \\
\hline Median & 0,92 \\
\hline Minimum & 0,00 \\
\hline Maximum & 5,41 \\
\hline \hline Gesamt & 27 \\
\hline
\end{tabular}

* Ohne Extremwerte.

Tab. 44 macht deutlich, daß sich mit jener Praxis in der Mehrheit der einschlägigen $(28)^{394}$ Fälle ein durchaus beträchtlicher zeitlicher Aufschub

393 Vgl. oben Ziff. 3.2 und Anm. 242.

394 Ausgenommen ist ein Fall, bei dem es sich um den Entwurf eines Teilgenehmigungsbescheids handelte. 
verbindet. Dieser setzt sich aus der dem Antragsteller eingeräumten Äußerungsfrist und der Zeit, die im Falle von Kritik am Entwurf für die abschlieBende Klärung der umstrittenen Punkte - bei bemängelten nicht-immissionsschutzrechtlichen Auflagen unter Beteiligung der zuständigen Fachbehörden - aufgewandt wird.

Der hinzukommende Zeitbedarf (Häufigkeitsverteilung über verschiedene Zeitklassen, oberer Teil der Tabelle) bewegt sich lediglich bei weniger als einem Fünftel $(17,9 \%)$ der betroffenen Verfahren innerhalb einer Zweiwochenfrist. In knapp der Hälfte der Fälle übersteigt er jedoch die Monatsfrist (Kumulation der letzten beiden Kategorien im oberen Teil), davon wiederum zur Hälfte sogar die Dreimonatsfrist. Das wirkt sich entsprechend auf die durchschnittliche Dauer aus, die knapp anderthalb $(1,44)$ Monate beträgt (unterer Teil der Tabelle).

Die zusätzliche Phase eines zur »Diskussion « gestellten Genehmigungsentwurfs erhöht - bezogen auf die Gesamtheit der Verfahren, also einschließlich der übrigen Fälle ohne Entwurf - die durchschnittliche Gesamtverfahrensdauer beim GAA-N um rd. drei Wochen (=0,67 Mon. bzw. 20,4 Tage) gegenüber 5 Tagen (=0,16 Mon.) beim LRA-1. ${ }^{395}$

\subsubsection{Widerspruchsbearbeitung (»Ñachphase»)}

Im Falle eines Widerspruchs durch den Antragsteller findet das Verfahren auf der Ebene der unteren Verwaltung mit der Entscheidung der Genehmigungsbehörde über eine mögliche Abhilfe seinen Abschluß. ${ }^{396}$ Vom Gebrauch dieses Rechtsmittels war, wie gezeigt (Ziff. 3.4.1), beim GAA-N rd. ein Viertel der Verfahren betroffen und damit ein deutlich größerer Anteil als beim LRA-1 mit rd. einem Siebtel.

Tab. 45 zeigt die Häufigkeitsverteilung der für die Widersprüche in Anspruch genommenen Bearbeitungsdauern über verschiedene Zeitklassen, Tab. 45.1 die zugehörigen statistischen Parameter.

395 Der Verlängerungseffekt beim LRA-1 ist allerdings nicht repräsentativ (und nur der Vollständigkeit halber aufgeführt), da er lediglich auf zwei - zudem Ausnahmen darstellenden - Fällen beruht.

396 Wie oben (Ziff. 4.2.1) bereits angesprochen, wird die Bearbeitung der Widersprüche bei der übergeordneten Widerspruchsbehörde nicht mehr dem Verfahren bei der unteren Genehmigungsbehörde zugerechnet. 
Tab. 45

Dauer der Widerspruchsbearbeitung durch die Genehmigungsbehörde (Widerspruchseinlegung bis Widerspruchsentscheidung) (Häufigkeitsverteilung)*

\begin{tabular}{|l|c|c|c|}
\cline { 2 - 4 } \multicolumn{1}{c|}{} & GAA-N & LRA-I & LRA-2 \\
\hline Bis zu einem Monat & 2 & 0 & 0 \\
& $13,3 \%$ & $0,0 \%$ & $* *$ \\
\hline Über einen bis drei Monate & 5 & 3 & 1 \\
& $33,3 \%$ & $50,0 \%$ & $* *$ \\
\hline Über drei bis sechs Monate & 1 & 2 & 0 \\
& $6,7 \%$ & $33,3 \%$ & $* *$ \\
\hline Über sechs Monate** & 7 & 1 & 0 \\
\hline \hline Widerspruchsverfahren gesamt & $46,7 \%$ & $16,7 \%$ & $* *$ \\
\hline
\end{tabular}

* Angesichts der niedrigen absoluten Grundgesamtheiten an Widersprüchen dient die Prozentuierung lediglich einer näherungsweisen (heuristischen) Vergleichbarkeit der auf die einzelnen Kategorien entfallenden absoluten Häufigkeiten. Beim LRA-2 unterbleibt sie (nur 1 Fall insgesamt).

** Maximaler Wert beim GAA-N: 17,5 Mon., LRA-1: 6,3 Mon., LRA-2: 2,6 Mon.

In der Häufigkeitsverteilung (Tab. 45) finden sich beim GAA-N gegenüber dem LRA-1 höhere Anteile in der untersten (höchstens ein Monat) und in der obersten Zeitklasse (mehr als sechs Monate) und entsprechend niedrigere Anteile in den beiden mittleren Zeitklassen (mehr als ein Monat bzw. mehr als drei bis sechs Monate). Wegen der geringen absoluten Fallzahlen bleiben aber alle Unterschiede unterhalb der Signifikanz. Das gilt auch für die Differenz in Höhe von 2,6 Monaten bei der durchschnittlichen Bearbeitungsdauer der Widersprüche, die sich beim GAA-N auf 6,7 Monate und beim LRA-1 auf 4,1 Monate beläuft (Tab. 45.1). ${ }^{397}$

Die unter der Verantwortung der Genehmigungsbehörde vollzogene Widerspruchsphase erhöht - bezogen auf die Gesamtheit der Verfahren, also einschließlich der mehrheitlichen Fälle ohne Widerspruch - die durchschnittliche Gesamtverfahrensdauer beim GAA-N immerhin um rd. eindreiviertel $(1,74)$ Monate, beim LRA-1 auf Grund der deutlich geringeren Anzahl der Widerspruchsfälle (und auch deren durchschnittlich geringeren Bearbeitungszeit) dagegen nur um rd. einen halben $(0,57)$ Monat.

$397 \mathrm{t}=1,059<\mathrm{t}_{(0,90 ; 19)}=1,328$ bei $\mathrm{F}=8,37<\mathrm{F}_{(0,99 ; 14,5)}=9,77$ bzw. $>\mathrm{F}_{(0,975 ; 14,5)}=$ 6,46. - Dieser Unterschied könnte auch Ausdruck der größeren Verwaltungsroutine der Verwaltungsfachleute sein. Dieses Ergebnis kann sich allerdings auf nur wenige (sechs) Fälle stützen. 
Tab. 45.1

Dauer der Widerspruchsbearbeitung durch die Genehmigungsbehörde (Widerspruchseinlegung bis Widerspruchsentscheidung) (statistische Parameter in Monaten)

\begin{tabular}{|l|c|c|c|}
\cline { 2 - 4 } \multicolumn{1}{c|}{} & GAA-N & LRA-1 & LRA-2 \\
\hline Arith. Mittel & 6,72 & 4,10 & 2,59 \\
\hline Varianz & 34,09 & 4,07 & $* *$ \\
\hline Median & 5,57 & 4,00 & $* *$ \\
\hline Minimum & 0,79 & 1,97 & 2,59 \\
\hline Maximum & 17,54 & 6,26 & 2,59 \\
\hline \hline Gesamt & 15 & 6 & 1 \\
\hline
\end{tabular}

\subsection{Fazit}

Eine zusammenfassende Übersicht über die Relationen der durchschnittlichen Dauern der einzelnen Verfahrensabschnitte untereinander und zur Gesamtdauer gibt - in chronologischer Abfolge - Tab. 46. Ihr kann man leicht entnehmen, welche Phasen sich durch vergleichsweise hohen Zeitbedarf auszeichnen und inwieweit sich dabei die beiden Genehmigungsmodelle voneinander unterscheiden.

Das mit Abstand größte Zeitkontingent verbucht die Phase der Vorantragsberatung (1). Das gilt für beide Genehmigungsmodelle gleichermaBen, wenn sich auch diese Phase beim LRA-1 mit einer Dauer von einem guten Dreivierteljahr (9,6 Mon.) deutlich länger hinzog als beim GAA-N mit einer Dauer von einem knappen halben Jahr (5,6 Mon.). Dieser, in den herkömmlichen Berechnungen zur Verfahrensdauer regelmäßig außen vor gelassene, als »inoffiziell « behandelte Verfahrensabschnitt währte bei beiden Ämtern in etwa ebenso lang - beim GAA-N etwas kürzer, beim LRA1 etwas länger - wie die anschließende »offizielle « Laufzeit ab Antragseingang (8).

Den zweithöchsten Zeitanteil verbrauchten die Fachbehörden im Rahmen der Erarbeitung ihrer Stellungnahmen (5). ${ }^{398}$ Er liegt bei beiden Modellen bei rd. vier (GAA-N: 3,95) bzw. knapp vier (LRA-1: 3,65) Monaten. Damit kostete diese Phase, die sich als einzige einem nennenswerten Einfluß der Genehmigungsbehörde praktisch entzieht, in etwa ebenso viel Zeit - beim 
GAA-N etwas mehr, beim LRA-1 etwas weniger - wie alle übrigen Verfahrensabschnitte des »offiziellen « Zeitrahmens zusammengenommen. ${ }^{399}$

Tab. 46

Übersicht über die durchschnittlichen Dauern der einzelnen Verfahrensabschnitte (arithmetische Mittel in Monaten)*

\begin{tabular}{|l|c|c|c|}
\cline { 2 - 4 } \multicolumn{1}{c|}{} & GAA-N & LRA-1 & LRA-2 \\
\hline (1) Vorantragsphase** & 5,60 & 9,58 & 5,26 \\
\hline (2) Vollständigkeitsprüfung & 0,40 & 0,26 & 0,67 \\
\hline (3) Vorbereitung Fachbehördenbeiziehung & 0,23 & 0,23 & 0,20 \\
\hline (4) Durchführung Fachbehördenbeiziehung & 0,18 & 2,36 & 1,50 \\
\hline (5) Fachbehördl. Abfassung d. Stellungnahmen & 3,95 & 3,65 & 2,26 \\
\hline (6) Erstellung des Genehmigungsbescheids & 1,68 & 0,86 & 1,43 \\
\hline \hline (7) Gesamtdauer ab Vorantragsberatung *** & 11,68 & 16,46 & 10,81 \\
\hline (8) Gesamtdauer ab Antragseingang*** & 6,63 & 8,00 & 6,50 \\
\hline \hline (9) Widerspruchsbearbeitung**** & 1,74 & 0,57 & 0,11 \\
\hline \hline (10) Effektive Gesamtdauer***** & 13,60 & 17,03 & 10,92 \\
\hline
\end{tabular}

* Ohne Extremwerte.

** Von Tab. 39.1 abweichende Werte, da in die hier wiedergegebenen Mittelwerte auch die Verfahren ohne Vorantragsberatungen eingegangen sind.

*** Die Werte sind Tab. 38.11 bzw. 37.11 entnommen und liegen wegen Rundungseffekten unter denen, die sich aus der Addition der hier wiedergegebenen Werte für die einzelnen Verfahrensabschnitte ergeben.

**** Von Tab. 45.1 abweichende Werte, da in die hier wiedergegebenen Mittelwerte auch die (bei allen drei Ämtern die große Mehrheit ausmachenden) Verfahren ohne Widerspruch eingegangen sind.

***** Dauer ab Vorantragsberatung bis Widerspruchsbescheid.

398 Dabei ist zu berücksichtigen, daß es sich hier um die Mindestdauer der Fachbehördenbeteiligung handelt, nämlich den Zeitraum zwischen der zuletzt ergangenen Aufforderung zur fachbehördlichen Äußerung und der zuletzt bei der Genehmigungsbehörde eingegangenen Stellungnahme (vgl. oben Ziff. 4.3.3.2 mit den Tab. 42.2 bis 42.22 ).

399 Diese Zeit ergibt sich aus Phase (8) abzüglich Phase (5). Sie beträgt 2,98 Mon. beim GAA-N und 4,35 Mon. beim LRA-1. 
Alle der fachbehördlichen Erarbeitung der Stellungnahmen vorausgehenden bzw. nachfolgenden Stadien dauerten bei beiden Ämtern jeweils weniger als vier Wochen, wobei für jedes Amt eine Ausnahme zu machen ist. Sie betrifft beim LRA-1 die Phase der Fachbehördenbeiziehung (4), für die es knapp zweieinhalb $(2,36)$ Monate benötigte, bis auch das letzte der im konkreten Fall zu beteiligenden Fachämter zur Stellungnahme aufgefordert war. Das GAA-N erledigte dagegen diesen Schritt in weniger als einer Woche (in 5,5 Tagen oder 0,18 Mon.). Die andere Ausnahme betrifft die Phase der Erarbeitung des abschließenden Genehmigungsbescheids (6), die bei der niedersächsischen Behörde gut sieben Wochen (oder 1,68 Mon.) dauerte und damit rd. doppelt so lange wie beim baden-württembergischen LRA-1 mit nur dreieinhalb Wochen (oder 0,86 Mon.). Der längere Bearbeitungszeitraum des GAA-N ist dabei in erster Linie der dort verbreiteten Übung zuzuschreiben, vorab dem Antragsteller eine Entwurfsfassung mit Gelegenheit zur Gegenäußerung zu übermitteln.

Zugespitzt läßt sich die Analyse zum Zeitverbrauch einzelner Verfahrensabschnitte wie folgt zusammenfassen. Geht man vom Antragseingang als dem faktisch »offiziellen« Beginn des Genehmigungsverfahrens aus, fällt in beiden Genehmigungsmodellen das mit Abstand größte Zeitkontingent auf die Erarbeitung der von den Fachbehörden abzugebenden Stellungnahmen, d. h. auf den von der Genehmigungsbehörde am wenigsten zu »steuernden « Arbeitsschritt. Etwa gleich viel Zeit benötigten alle übrigen, zugleich weitgehend dem Verfügungsbereich der Genehmigungsbehörde unterliegenden Verfahrensabschnitte. Von diesen belasteten Vollständigkeitsprüfung und Vorbereitung der Fachbehördenbeteiligung das Zeitbudget vergleichsweise nur gering. Dabei ist allerdings zu berücksichtigen, daß in beiden Modellen ein Teil der von der Genehmigungsbehörde zu leistenden Prüfung des anstehenden Vorhabens auf seine Genehmigungsfähigkeit hin zumeist bereits in der dem offiziellen Antragseingang vorgeschalteten »inoffiziellen« Beratungsphase absolviert werden konnte, die im Ergebnis in etwa ebenso lang dauerte wie das nachfolgende »offizielle« Verfahren. Der Zeitverbrauch für die beiden restlichen zwei Phasen dieser »offiziellen « Laufzeit - Durchführung der Fachbehördenbeiziehung und Erstellung des Genehmigungsbescheids - fällt bei den beiden Modellen deutlich unterschiedlich aus. Nimmt im Süden die Fachbehördenbeiziehung das zweitgrößte Zeitkontingent in Anspruch, ist es im Norden der Genehmigungsbescheid.

Unterschiede im Zeitverbrauch zwischen den beiden Modelle betreffen mithin ausschließlich von der Genehmigungsbehörde zu verantwortende Verfahrensabschnitte und nicht das weitgehend von den Fachbehörden determinierte Stadium der Erarbeitung ihrer Stellungnahmen. ${ }^{400}$ Entsprechend liegt es nahe, sie in erster Linie der jeweils modellbedingten institu- 
tionellen Trennung bzw. Zentralisierung von Genehmigungs- und immissionsschutzrechtlicher Fachkompetenz zuzuschreiben.

Die auf der Ebene der Durchschnittswerte aufgezeigten Zusammenhänge lassen sich durch eine Korrelations- und Regressionsanalyse, die die einzelnen Dauern auf der Basis der nicht-aggregierten, individuellen Werte je Verfahren miteinander in Beziehung setzt, absichern und präzisieren.

Die Korrelationsanalyse ermöglicht es, Stärke (Korrelationskoeffizient r mit den Werten zwischen 0 und 1) und Richtung des Zusammenhangs (positives oder negatives Vorzeichen von r) jeweils zwischen zwei Variablen zu bestimmen. Die Interdependenz (oder Kovarianz) zwischen zwei Variablen ist umso größer, je mehr sich der Koeffizient $r$ dem Wert 1 annähert. Sie ist positiv, wenn die beiden Variablen in derselben Richtung, und negativ, wenn sie in entgegengesetzter Richtung kovariieren. ${ }^{401}$ Die berechneten Zusammenhänge zwischen einzelnen Variablen lassen sich anschaulicher darstellen, wenn man statt des Koeffizienten $r$ dessen quadrierten Wert $r^{2}$ zugrunde legt. Dieser Wert gibt an, wieviel Prozent der Varianz einer Variablen durch die andere Variable auf Grund ihrer linearen Beziehung erklärt werden kann. ${ }^{402}$

Tab. 46.1 zeigt die Korrelationen der einzelnen Verfahrensabschnitte (1) bis (6) mit der Gesamtdauer, jeweils ab Vorantragsberatung (7) und ab Antragseingang (8), auf der Basis der erklärten Varianzen $r^{2} .403$

Wie aus der Tabelle hervorgeht, korreliert die Gesamtdauer der Verfahren ab Antragseingang (8) beim LRA-1 am stärksten mit der Dauer der Fachbehördenbeiziehung (4). Auf diese Phase lassen sich - für sich genommen $-67,40 \%$ der Varianz der Gesamtdauer zurückführen. Beim GAA-N dagegen beträgt der entsprechende Wert lediglich 5,33\%. Dort ist es vielmehr die Phase der fachbehördlichen Erarbeitung der Stellungnahmen (5), die am stärksten mit der Gesamtdauer nach Antragseingang korreliert und $58,06 \%$ von deren Varianz gegenüber lediglich $12,95 \%$ beim LRA-1 er-

400 Auch dieses Ergebnis fügt sich in den schon den (inhaltlichen) Verfahrensverlauf kennzeichnenden Befund, nach dem sich in beiden Genehmigungsmodellen die Mitwirkung der (modellunabhängigen) Fachbehörden weitgehend ähnlich gestaltete.

401 Bei positiver Korrelation gehen höhere Werte der einen Variablen überwiegend mit höheren Werten auch der anderen Variablen einher, bei negativer Korrelation treffen höhere Werte der einen Variablen überwiegend mit niedrigeren Werten der anderen zusammen.

402 Einem Anteil von $100 \%$ erklärter Varianz entspricht eine Korrelation von $r=1$. In diesem Fall ist die eine Variable vollständig (linear) durch die andere determiniert, wobei die Determiniertheit nicht nur in positiver, sondern auch in negativer Richtung gegeben sein kann.

403 Mit dem Bezug auf den $\mathrm{r}^{2}$-Wert ist insofern ein Nachteil verbunden, als er auch bei negativem Vorzeichen des Ausgangswerts r stets positiv ist, so daß die ursprüngliche Information über die Richtung des Variablenzusammenhangs verlorengeht. Deshalb sind die Korrelationskoeffizienten r zusätzlich in Tab. 46.11-A im Anhang wiedergegeben. 
klärt. Alle übrigen Verfahrensabschnitte tragen - wiederum jeweils für sich genommen - in beiden Modellen weitaus weniger zur Erklärung der Gesamtdauer bei. Unter ihnen kommt beim LRA-1 der gerade genannten Abfassung der Stellungnahmen durch die Fachbehörden mit einem Erklärungsanteil von $12,95 \%$ und beim GAA-N der Erstellung des Genehmigungsbescheids mit einem Erklärungsanteil von 12,75\% noch ein gewisses Gewicht zu. Der Beitrag der restlichen Phasen liegt jeweils unter 10\%, ausgenommen die Vorantragsphase, auf die abschließend gesondert einzugehen ist.

Tab. 46.1

Korrelationen der einzelnen Verfahrensabschnitte mit der Gesamtdauer $-r^{2}$-Werte (erklärte Varianzen ) -*

\begin{tabular}{|c|c|c|c|}
\hline & \\
\hline & & $\begin{array}{c}\text { Gesamt- } \\
\text { dauer ab Vor- } \\
\text { antragsbear- } \\
\text { beitung (7) }\end{array}$ & $\begin{array}{c}\text { Gesamt- } \\
\text { dauer ab An- } \\
\text { tragseingang } \\
\text { (8) }\end{array}$ \\
\hline (1) Vorantragsphase & $\begin{array}{l}G A A-N \\
L R A-1\end{array}$ & $\begin{array}{l}r^{2}=61,94 \% \\
r^{2}=61,39 \%\end{array}$ & $\begin{array}{l}r^{2}=11,51 \% \\
r^{2}=1,21 \%\end{array}$ \\
\hline (2) Vollständigkeitsprüfung & $\begin{array}{l}G A A-N \\
L R A-1\end{array}$ & $\begin{array}{l}r^{2}=0,00 \% \\
r^{2}=0,28 \%\end{array}$ & $\begin{array}{l}\mathrm{r}^{2}=0,36 \% \\
\mathrm{r}^{2}=6,95 \%\end{array}$ \\
\hline (3) Vorbereitung Fachbehördenbeiziehung & $\begin{array}{l}G A A-N \\
L R A-1\end{array}$ & $\begin{array}{l}r^{2}=0,59 \% \\
r^{2}=0,56 \%\end{array}$ & $\begin{array}{l}r^{2}=1,35 \% \\
r^{2}=0,45 \%\end{array}$ \\
\hline (4) Durchführung Fachbehördenbeiziehung & $\begin{array}{l}G A A-N \\
L R A-1\end{array}$ & $\begin{array}{l}r^{2}=4,53 \% \\
r^{2}=17,04 \%\end{array}$ & $\begin{array}{l}r^{2}=5,33 \% \\
r^{2}=67,40 \%\end{array}$ \\
\hline (5) Fachbehördl. Abfassung d. Stellungnahmen & $\begin{array}{l}G A A-N \\
L R A-1\end{array}$ & $\begin{array}{l}r^{2}=39,15 \% \\
r^{2}=2,21 \%\end{array}$ & $\begin{array}{l}r^{2}=58,06 \% \\
r^{2}=12,95 \%\end{array}$ \\
\hline (6) Erstellung des Genehmigungsbescheids & $\begin{array}{l}G A A-N \\
L R A-1\end{array}$ & $\begin{array}{l}r^{2}=7,30 \% \\
r^{2}=0,00 \%\end{array}$ & $\begin{array}{l}r^{2}=12,75 \% \\
r^{2}=8,67 \%\end{array}$ \\
\hline (7) Gesamtdauer ab Vorantragsberatung*** & $\begin{array}{l}\text { GAA-N } \\
L R A-1\end{array}$ & $\begin{array}{l}* * * \\
* * *\end{array}$ & $\begin{array}{l}\mathrm{r}^{2}=65,06 \% \\
\mathrm{r}^{2}=21,03 \%\end{array}$ \\
\hline
\end{tabular}

* Berechnung der Koeffizienten unter paarweisem Ausschluß von Extremwerten. Die Mindestzahl paarweise eingeschlossener Fälle beträgt beim GAA-N 52 und beim LRA-1 39 Fälle.

Die Ergebnisse der Korrelationsanalyse verweisen auf einen weiteren, deutlichen Unterschied zwischen den beiden Genehmigungsmodellen. Die (jeweils behördeninternen) Variationen der (ab Antragseingang berechne- 
ten) Gesamtdauer ${ }^{404}$ sind beim niedersächsischen GAA-N in erster Linie auf (co-) variierende Dauern einer von diesem nur wenig beeinflußbaren Phase, der fachbehördlichen Erarbeitung der Stellungnahmen, zurückzuführen und erst in zweiter Linie - und dies mit deutlichem Abstand - auf Variationen der in der eigenen Hand liegenden Zeitspanne für die Erstellung des Genehmigungsbescheids. Beim baden-württembergischen LRA-1 dagegen sind die Variationen der Gesamtdauer vor allem durch (co-) variierenden Zeitaufwand für eine der eigenen Verfügung unterstehenden Phase, der Beiziehung der Fachbehörden, bestimmt. Von seiten der Genehmigungsbehörde steuerbare Beschleunigungspotentiale sind von daher im Norden hauptsächlich bei der Erstellung des Genehmigungsbescheids und im Süden bei der Beiziehung der Fachbehörden gegeben. Das größere, aber von den Genehmigungsbehörden nur wenig beeinflußbare Beschleunigungspotential liegt aber bei den Fachbehörden. Deren für die Erarbeitung der Stellungnahmen in Anspruch genommener Zeitverbrauch, der in beiden Genehmigungsmodellen in etwa gleich groß ist, macht jeweils das mit Abstand gegenüber den übrigen Phasen größte Kontingent an der »offiziellen « Gesamtdauer aus.

Beschleunigungspotentiale lassen sich freilich auch bei der »inoffiziellen « Vorantragsphase vermuten. Deren in beiden Modellen im Vergleich zur »offiziellen« Verfahrenslaufzeit erhebliche Dauer beeinflußt allein auf Grund ihres (absoluten) zeitlichen Gewichts auch die von der ersten Vorantragsberatung berechnete Gesamtdauer der Verfahren (7) bei beiden Ämtern in gleich hohem Maße mit 61,94\% (GAA-N) und 61,39\% (LRA-1) erklärter Varianz. Insofern ist dieses Ergebnis nicht überraschend. Weniger zu erwarten war dagegen, daß die Dauer der Vorantragsphase nur in einem schwachen Zusammenhang mit der anschließenden Verfahrensdauer ab Antragseingang (8) steht. Beim GAA-N beläuft sich die erklärte Varianz auf nur $11,51 \%$ bei positiver Korrelation, d.h. längere Vorantragsphasen ziehen eher auch längere »offizielle « Gesamtdauern nach sich. ${ }^{405}$ Beim LRA-1 ist der Zusammenhang noch schwächer ausgeprägt mit lediglich $1,21 \%$ erklärter Varianz, wobei der Zusammenhang tendenziell invers ist, d.h. längeren Gesamtdauern gingen eher kürzere Vorantragsphasen bzw. kürzeren Gesamtdauern gingen eher längere Vorantragsphasen voraus.

404 Die Variation der Gesamtdauer ist grundsätzlich unabhängig vom jeweiligen Durchschnittswert der Dauer, die sich aus den mittleren Dauern der einzelnen Verfahrensabschnitte (bzw. deren Summe) ergibt (s. Tab. 46). Es ist also durchaus möglich, daß die Variation auch identischer durchschnittlicher Gesamtdauern durch unterschiedliche Dauern der einzelnen Phasen in unterschiedlichem Ausmaß bestimmt sein kann.

405 Vgl. hierzu die Korrelationskoeffizienten in Tab. 46.11-A im Anhang. 
Als eine Art abschließende Bilanz des Modellvergleichs werden nachfolgend diejenigen Untersuchungsergebnisse zusammengeführt, die sich als modellbedingte Charakteristika der baden-württembergischen Genehmigungspraxis einerseits und der niedersächsischen Genehmigungspraxis andererseits herauskristallisiert haben, ging doch die Untersuchung von der Fragestellung aus, wie sich die unterschiedlichen organisatorischen und qualifikatorischen Rahmenbedingungen auf den Verfahrensverlauf und die Verfahrensergebnisse, wie sie in den ausgewerteten Akten dokumentiert sind, auswirken. Im Hinblick darauf wurden zwei Stufen des $»$ Gesetzesvollzugs « unterschieden (vgl. Kap. I), eine erste Vollzugsstufe i. S. der Befolgung verfahrensrechtlicher Bestimmungen sowie der inhaltlichen Festlegung der Betreiberpflichten durch Auflagen und Bedingungen, auf die sich die vorliegende Untersuchung konzentriert, von einer zweiten Vollzugsstufe, bei der die »eigentliche « Umsetzung bzw. Durchsetzung der im Genehmigungsbescheid getroffenen Anforderungen durch den Betreiber bzw. durch die Vollzugsbehörde erfolgt.

Auf modellspezifische Besonderheiten zwischen der nord- und süddeutschen Lösung weisen zunächst entsprechende Befunde zu Art, Häufigkeit und Ausgang von fachlich-technischen bzw. verfahrensrechtlichen Kontroversen (sowohl in immissionsschutzrechtlicher als auch in nicht-immissionsschutzrechtlicher Hinsicht) zwischen den am Verfahren Hauptbeteiligten hin. Die im Süden vorfindbare Ausgangsbedingung begünstigt eine Art »moral division of labour « zwischen der gegenüber »Kundeninteressen « der im Wirtschaftsraum des eigenen Landkreises tätigen Unternehmen sich aufgeschlossener zeigenden Genehmigungsbehörde (LRA-1) und der zugehörigen technischen Fachbehörde (GAA-1), die nicht unter dem unmittelbaren Erwartungsdruck der Antragsteller bzw. Anlagenbetreiber steht und insofern relativ »unbeeindruckt« die rechtlich normierten Standards mit Hinweis auf ihre Fachkompetenz zur Geltung bringt. Auch wenn - wie dies der Vergleich mit der norddeutschen Lösung zeigt - die baden-württembergische Lösung mit ihrer Aufspaltung in Genehmigungs- und technische Fachbehörde von vornherein für zusätzlichen Konfliktstoff sorgt (vgl. Tab. 22), so entzünden sich die im Süden beobachtbaren Konflikte in besonderem Maße an immissionsschutzrechtlichen Fragen (Tab. 22.1; Tab. 23). Im Durchschnitt aller Verfahren (Tab. 23.1) gab es dort drei Mal so viele Kontroversen dieser Art wie im Norden. (Daß dieses Nord-Süd-Gefälle nicht auf modellunabhängige Randbedingungen zurückzuführen ist, wurde überprüft). Differenziert man die für das Nord-Süd-Gefälle verantwortlichen 
immissionsschutzrechtlichen Belange nach Konfliktgegenständen in fachlich-technische Kontroversen um die Anlagenausstattung (bzw. um den Anlagenbetrieb) und verfahrensrechtliche Kontroversen, dann zeigt sich allerdings, daß bei beiden Modellen die verfahrensrechtlichen Kontroversen überwiegen (Tab. 24). Dieser Konfliktbereich betrifft den von der Genehmigungsbehörde im Süden beanspruchten »ureigenen « Kompetenzbereich, was - zieht man zum Vergleich die fachlich-technischen Kontroversen heran - in einer größeren Konfliktbereitschaft gegenüber der technischen Fachbehörde zum Ausdruck kommt (Tab. 24.1). Insgesamt erweist sich bei den verfahrensrechtlichen Kontroversen zwischen Genehmigungsbehörde und technischer Fachbehörde die Gewinn-Verlust-Bilanz, die deren Ausgang zugunsten bzw. zu Lasten des Antragstellers/Betreibers spiegelt, als beinahe ausgeglichen. Ging es der technischen Fachbehörde dabei vor allem um Fragen der grundsätzlichen Genehmigungsbedürftigkeit (mit eingeschlossen Fragen zur Art der Genehmigung sowie dazu, welche Anlagenteile und Nebeneinrichtungen von ihr erfaßt werden sollen) und um die Durchsetzung von Sanierungserfordernissen (z.B. i.S. der rechtlichen Absicherung von Fristsetzungen entweder in der Form der nachträglichen Anordnung oder in der Form des öffentlich-rechtlichen Vertrags), so setzte sich bei den genannten Kontroversen die Genehmigungsbehörde in erster Linie mit dem Verlangen der technischen Fachbehörde auscinanander, Verwaltungszwangsmaßnahmen (wie Stillegungsverfügung oder Bußgeldfestsetzung) zu verhängen. Zugespitzt formuliert, könnte man sagen, daß die Genehmigungsbehörde vor allem dann dazu tendierte, von ihrer formellen Entscheidungskompetenz Gebrauch zu machen, wenn fachtechnischer Sachverstand nicht unbedingt gefragt war. Überhaupt vermittelt eine an der Vollzugswirklichkeit sich orientierende Betrachtungsweise ein Bild, bei dem die technische Fachbehörde im Süden die Rolle des faktischen »Weichenstellers « bei dem immissionsschutzrechtlichen Genehmigungsverfahren einnimmt, wohingegen die formell zuständige Genehmigungsbehörde eher in der Aufgabe der »prozeduralen Steuerung « aufgeht, sofern man hierunter eine auf den Verfahrensablauf zentrierte Koordinierung der Beteiligten (im Sinne der Verteilung von Unterlagen, der Setzung von Fristen und deren Anmahnung) versteht. So gesehen, hat sich im Süden zwischen Landratsamt und »seinem « zuständigen Gewerbeaufsichtsamt eine Form der tatsächlichen Zusammenarbeit - im Sinne der von Friedberg (1995, S. 9) bezeichneten $» l o k a l e n$ Ordnung $\aleph^{406}$ - herausgebildet, bei der die von der Formalstruktur vorgegebene Abfolge resp. Zuordnung »umgekehrt« wird. ${ }^{407}$ So ist es die technische Fachbehörde, die mit ihren fachlichen Vorgaben und der Aufforderung, diese auch förmlich umzusetzen, die Geneh-

406 1995, S. 3 u. S. 9.

407 Vergleichbare Befunde zur »wirklichen Funktionsweise « von Organisationen finden sich z.B. bei Friedberg 1995, S. 145ff., S. 367ff. Vgl. aber auch Freudenberg 1993, 
migung(serteilung) vorstrukturiert und dadurch der Genehmigungsbehörde eine Doppelfunktion zuweist: Zum einen im Sinne einer für die technische Fachbehörde tätigen »Geschäftsstelle« bzw. »Postverteilungsagentur«, zum anderen im Sinne eines »Notariats «, das in der Regel den inhaltlichen Vorgaben der technischen Fachbehörde formal rechtliche Qualität verleiht und diesem dabei sogar noch vielfach die juristische »Richtigkeitskontrolle ${ }^{408}$ überläßt. ${ }^{409} \mathrm{Daß}$ sich die »wirklichen « Entscheidungsabläufe nicht an die formal zugewiesenen Kompetenzen halten, veranschaulichen vor allem jene 16 Fälle verfahrensrechtlicher Kontroversen, bei denen die formal zuständige Genehmigungsbehörde ihre in Aussicht genommene Entscheidung der technischen Fachbehörde zur »Billigung « vorlegte, deren Auffassung sie dann auch übernahm, obwohl sie dadurch ihre ursprünglich eingenommene Position revidieren mußte. Die technische Fachbehörde im Süden gleicht auf diese Weise die ihr fehlende formale Genehmigungskompetenz aus. Sie beschränkt sich faktisch nicht auf die Rolle einer der Genehmigungsbehörde zuarbeitenden Fachbehörde, sondern gibt sich dieser gegenüber als »Hüterin « der vom Gesetz geforderten Standards. Für einen gesetzestreuen Vollzug des BImSchG auf der ersten Vollzugsstufe bietet, so gesehen, das baden-württembergische Organisationsmodell mit seiner Kompetenzaufteilung die weitaus günstigeren Voraussetzungen. ${ }^{410}$

Die niedersächsische Lösung mit dem zugleich als Genehmigungsbehörde fungierenden Gewerbeaufsichtsamt (GAA-N) muß infolge der fehlenden organisatorischen Arbeitsteilung zwischen Genehmigungsbehörde und technischer Fachbehörde auf den nützlichen Mechanismus wechselseitiger legitimatorischer Entlastung bei der »Umsetzung« umweltschutzrechtlicher Vorgaben auf der ersten Vollzugsstufe verzichten. Aus dieser dem GAA-N im Norden zugewiesenen Doppelfunktion als Genehmigungsund technische Fachbehörde resultiert eine von der süddeutschen abweichende Genehmigungspraxis, die - so die Befunde in Tab. 22 - erheblich weniger sachliche Kontroversen aufweist. Auch wenn im Unterschied zu

S. 235, mit dem Hinweis, daß sich die Bezirksregierungen faktisch zur »Bürogemeinschaft regionaler Dienststellen von Fachverwaltungen« entwickelt hätten.

408 Vgl. zu diesem Begriff Mayntz 1997, S. 98: Richtigkeitskontrolle überprüft, inwieweit verbindliche Normen korrekt angewandt werden.

409 Die nahezu völlige Abhängigkeit der Verwaltungsbehörden von den technischen Fachbehörden konstatieren auch Mayntz et. al. 1978, S. 48f. und 255ff., während die theoretisch gegebene umgekehrte Abhängigkeit der Fachbehörden von der Verwaltungsbehörde sich auf die Vollzugspraxis eher nur marginal auswirke (S. 258f.).

410 Dieses Ergebnis steht der in der Literatur vorherrschend vertretenen Ansicht entgegen, wonach das Integrationsmodell (wie in Niedersachsen) gegenüber dem Trennungsmodell (wie in Baden-Württemberg) als die für die Durchsetzung von Umweltschutzanforderungen leistungsfähigere Organisationsform gilt. Vgl. hierzu etwa Mayntz et. al. 1978, S. 49f., ähnlich auch Dose 1995, S. 116f., ferner LübbeWolff 1996, S. 55f. u. S. 61ff. m.w.N., ihr zufolge muß das Trennungsmodell heute als Anachronismus angesehen werden (S. 62). 
Baden-Württemberg, wo die fachlichen Kontroversen zwischen Genehmigungs- und technischer Fachbehörde in der Regel aktenmäßig erfaßt werden, für Niedersachsen diese »aktenträchtige« Konstellation entfällt (so daß infolge der herangezogenen Methode die tatsächliche Konflikthäufigkeit nicht im vollen Umfang erfaßt sein dürfte), kann eine vorsichtige Interpretation in der erheblich höheren Rate an nicht kontrovers durchgeführten Verfahren einen Hinweis auf eine eher konsensorientierte Genehmigungspraxis sehen - eine Deutung, die sich für die niedersächsische Genehmigungsbehörde an Hand weiterer empirischer Befunde zusätzlich absichern läßt. Betrachtet man die Verfahren mit immissionsschutzrechtlichen Konflikten nämlich genauer, dann zeigt sich - so Tab. 24 -, daß bei beiden Modellen einerseits die verfahrensrechtlichen gegenüber den fachspezifischen Kontroversen überwiegen, andererseits in der Mehrzahl der verfahrensrechtlich kontroversen Fälle das niedersächsische GAA-N dem Betreiber entgegenkam bzw. ursprünglich erhobene Forderungen zurücknahm (Tab. 24.1) - allerdings ist der hierbei sich ergebende Unterschied zum süddeutschen Modell nicht signifikant. Eine ihre eher konsensorientierte Genehmigungspraxis unterstreichende »kundenfreundliche Politik « verfolgt die niedersächsische »Doppelbehörde« vor allem auf dem Gebiet ihrer genuinen Kompetenz als technischer Fachbehörde. Dies läßt sich zunächst daran ablesen, daß sie bereits bei der Vorantragsberatung sowohl hinsichtlich der Einforderung gutachtlicher Prüfbelege und Meßnachweise über Emissionen/Immissionen als auch bei Fristsetzungen für Sanierungsmaßnahmen insgesamt zurückhaltender ist (Tab. 11: Kategorie $a$ bzw. $b-$ hier erweisen sich die Unterschiede zum Süden als signifikant). Diese »Zurückhaltung « dürfte möglicherweise auf die Antizipation von Schwierigkeiten bei der Durchführung wirksamer Kontrolle im Hinblick auf die Einhaltung der Genehmigungsbedingungen in der zweiten Vollzugsstufe zurückzuführen sein, die ja im Norden - anders als im Süden - in die Zuständigkeit ein und derselben Behörde fällt, wie sich überhaupt die Erfüllung nur weniger Auflagen wirksamer kontrollieren läßt, als dies bei einer größeren Auflagenzahl pro Betrieb bei mehr oder weniger konstant bleibenden Ressourcen der Fall wäre. Indessen setzt sich diese »Zurückhaltung « fort bei der Erteilung von immissionsschutzrechtlichen Auflagen im Genehmigungsbescheid (Tab. 31 - wobei der jeweilige Unterschied zum Süden allerdings nur bei den geforderten baulich-technischen Schallschutzmaßnahmen signifikant ausfällt). Auch hinsichtlich der Forderungen nach Meß- und Prüfnachweisen (Tab. 32) verhält sich die niedersächsische Genehmigungsbehörde beim Genehmigungsbescheid zurückhaltender als ihr süddeutsches Pendant mit seiner eher prohibitiv orientierten Auflagenpraxis. Die hierzu ermittelten Unterschiede sind, von wenigen Ausnahmen abgesehen, zwar nicht signifikant, reichen jedoch beinahe alle knapp an die Signifikanzgrenze heran. Daher spricht einiges dafür, daß dieses Gefälle zwischen den 
beiden Modellen weniger auf unterschiedliche, anlagenspezifisch begründete Kontrollerfordernisse als auf unterschiedliche, jeweils spezifisch modellbedingte Genehmigungs- und Kontrollstrategien zurückzuführen ist. Gibt das baden-württembergische Modell demnach eher einer prophylaktisch-absichernden, den Antragsteller/Betreiber von vornherein auf bestimmte Schutz- und Prüfmaßnahmen verpflichtenden Strategie den Vorzug, so neigt das niedersächsische Modell eher zu einer zurückhaltenden Strategie mit einer »sparsameren « Verwendung immissionsschutzrechtlicher Auflagen und Nachweispflichten, wobei jedoch vornehmlich bei solchen Verfahren, bei denen für die festgelegten Emissions- bzw. Immissionsgrenzwerte keine Meßbelege für deren Einhaltung gefordert wurden, eine nach Inbetriebnahme der Anlage durchzuführende »Endabnahme« vorgesehen ist - im Sinne einer Gesamtprüfung der genehmigten Anlagenerrichtung bzw. -änderung (Tab. 32: vorletzte Kategorie). Auf diese Weise eröffnet sich die niedersächsische Genehmigungsbehörde für die zweite Vollzugsstufe einen größeren Handlungsspielraum. Es muß hier allerdings offen bleiben, inwieweit dieser auch tatsächlich ausgeschöpft wird. Die für das niedersächsische Modell eher typische konsensorientierte Vorgehensweise kommt auch in der Praxis zum Ausdruck, dem Antragsteller/Betreiber vor allem bei kontrovers verlaufenen Verfahren eine Entwurfsfassung des in Aussicht gestellten Genehmigungsbescheids zuzusenden, damit dieser hierzu Stellung nehmen kann (Tab. 27). Diese, die zeitlichen Ressourcen erheblich in Anspruch nehmende Praxis (Tab. 44) kann als eine Art »vorgezogenes Widerspruchsverfahren« angesehen werden. Tatsächlich zeigt sich, daß der Antragsteller/Betreiber diese durchaus »kundenfreundliche« Praxis dergestalt belohnt, daß er auf die Einlegung eines förmlichen Widerspruchs eher verzichtet (Tab. 29), selbst dann, wenn die Behörde die von ihm gewünschten Abänderungen der im Entwurf vorgesehenen Auflagen nur partiell vornimmt oder (in wenigen Fällen) gänzlich ablehnt. Förmliche Widersprüche betreffen denn auch vorzugsweise die Verfahren, in denen der Antragsteller/Betreiber keine Entwurfsfassung und mithin auch keine Gelegenheit zu (nicht-förmlichen) Einwendungen erhalten hat. Betrachtet man den Gesamtanteil an faktischen Einwendungen (gegen Entwurfsfassungen) und förmlichen Widersprüchen, so ist nicht zu verkennen, daß mit der Praxis, (aus der Sicht der Sachbearbeiter) kontrovers verlaufene Verfahren mit der Zusendung eines Genehmigungsentwurfs »abzuschlieBen«, ein gewisser Entlastungseffekt verbunden ist. So weist Tab. 33 auf seiten der niedersächsischen Genehmigungsbehörde ein Gesamtprotestpotential von $44,8 \%$ gegenüber einem solchen von $18,6 \%$ auf seiten der baden-württembergischen aus (dieser Unterschied ist hochsignifikant), wohingegen das Gefälle bei den Verfahren, in denen förmlicher Widerspruch erhoben wurde, mit einem Anteil von 25,9\% im Norden und von 14,0\% im Süden geringer ausfällt (dieser Unterschied liegt knapp unterhalb der Sig- 
nifikanzgrenze). Im Ergebnis dürfte der Eindruck nicht von der Hand zu weisen sein, daß die prophylaktische Wirkung der Entwurfsversendung darin besteht, die durch sie evozierte Neigung zur Beschwerde letztlich zu neutralisieren. Die niedersächsische Verfahrenspraxis ließe sich, verglichen mit der baden-württembergischen, demnach dahingehend charakterisieren, daß sie insgesamt als konsensorientierter erscheint, was auch daran ablesbar ist, daß sie in jedem zweiten Verfahren zum Verfahrensabschluß einen Genehmigungsentwurf übersendet. Dieser Umstand wie überhaupt die an einvernehmlichen Lösungen ausgerichtete Grundorientierung ermuntern Antragsteller indessen eher dazu, als daß sie diese davon abhielten, dann förmlichen Widerspruch einzulegen, wenn sie ihre Wünsche (Interessen) nicht berücksichtigt sehen. Doch zugleich wohnt der informellen Praxis der Entwurfszusendung eine »List « inne, als dadurch die von der generell konsensualen Verwaltungspraxis induzierte Neigung zum (förmlichen) Widerspruch wenigstens bis zu einem gewissen Grad wieder absorbiert wird.

Die Bevorzugung einer eher konsensorientierten Genehmigungspraxis im Norden könnte vorderhand weniger in der jeweiligen (modellbedingten) organisatorischen Trennung bzw. Zusammenfassung von Genehmigungsund technischer Fachkompetenz als solcher gründen als vielmehr in der damit einhergehenden unterschiedlichen beruflichen Qualifikation der Entscheidungsträger. Wenn nämlich davon auszugehen wäre, daß dem technisch ausgebildeten Verwaltungspersonal »die ingenieurmäßige Beratung vor Ort « eher zusagen dürfte als die mehr »bürokratischen Formen des umweltrechtlichen Vollzugs $«,{ }^{411}$ dann ließe sich vermuten, daß technisch qualifizierte Verwaltungskräfte auftretende Rechtsunsicherheiten eher mit einem konsensorientierten Vollzug kompensieren als für Verwaltungsaufgaben an entsprechenden Fachhochschulen eigens befähigte Verwaltungsfachleute, die im Umgang mit Recht versierter zu sein scheinen. Doch weder die mit der Aktenanalyse erzielten Resultate noch die jeweils vor Ort mit den Sachbearbeitern durchgeführten Interviews weisen in Richtung besagter Hypothese. Vielmehr herrscht der Eindruck vor, daß sich die Techniker bei den hier untersuchten vereinfachten Genehmigungsverfahren und dem weitgehend technisch geprägten Regelungsgegenstand des BImSchG hinsichtlich ihrer verfahrensrechtlichen Kompetenz durchaus mit den Verwaltungsfachleuten im Süden messen können. ${ }^{412}$

$* * *$

411 Lübbe-Wolff 1992, S. 231. Vgl. auch oben Anm. 8.

$412 \mathrm{Zu}$ einer ähnlichen Einschätzung kommt auch Dose 1997, S. $236 f$. 
Die vergleichende Analyse wurde auch auf die für die Durchführung der Verfahren beanspruchte Zeitspanne erstreckt. Die Ergebnisse dazu werden ebenfalls im Hinblick auf jeweils modellspezifische Charakteristika bilanziert. Auch wenn nicht zu übersehen ist, daß »Verfahrensdauer « inzwischen weitgehend den Status eines politischen Schlagworts eingenommen hat, so hat auf der anderen Seite der beharrlich diskutierte Kritikpunkt überlanger Verfahrensdauer in der jüngsten Vergangenheit wiederholt Gesetzesreformen ausgelöst bzw. als Argument gedient, solche einzufordern.

Greift man zunächst diese Kritik auf, dann zeigt sich, daß die hier untersuchten Verfahren ebenfalls zu lange dauern, wenn man sie an der gesetzlich vorgegebenen Regelfrist von drei Monaten mißt (zu den Details vgl. die Ausführungen in Kap. III, Ziff. 4.1). Legt man diese dreimonatige Regelfrist den drei sich grundsätzlich anbietenden Varianten zur Berechnung von Verfahrensdauern zugrunde - die vom Gesetzgeber favorisierte erste Variante läßt das Verfahren mit der »Vollständigkeit der Antragsunterlagen« beginnen, die zweite Variante mit dem »Antragseingang « und die dritte Variante mit der »ersten Vorantragsberatung «, wobei in allen drei Varianten der »Erlaß des Genehmigungsbescheids « das Verfahrensende markiert (vgl. Tab. 36.1 - Tab. 37.1 - Tab. 38.1) -, dann stellt sich heraus, daß bei beiden Organisationsmodellen innerhalb der vorgegebenen Regelfrist jeweils gerade einmal ein Viertel (erste Variante) bzw. ein Fünftel (zweite Variante) bzw. noch nicht einmal ein Zehntel (dritte Variante) der Verfahren abgeschlossen werden. Insofern schneidet bei diesem Vergleich die niedersächsische Vollzugspraxis, die ja mit dem auch über die Genehmigungskompetenz verfügenden GAA-N eine Behörde »einspart«, insgesamt nicht »besser « ab als das baden-württembergische Modell. Vor allem kann der Vergleich unterschiedlicher Berechnungsmodalitäten von Verfahrensgesamtdauern deutlich machen, daß die vom Gesetzgeber der Verwaltung zugestandene Verfahrensdauer (erste Variante) in etwa nur die Hälfte der für die Genehmigungserteilung tatsächlich in Anspruch genommenen Zeitspanne abzudecken vermag, insofern also höchst unrealistisch ist - wie überhaupt eine »angemessene « Genehmigungsdauer inhaltliche Mindeststandards zu berücksichtigen hätte.

Zieht man Tab. 38.2 heran, die die (den Vorantragszeitraum einbeziehende) Gesamtdauer nach Änderungs- und Neugenehmigungen differenziert, dann ergibt sich zwischen den beiden Modellen insofern ein gradueller Unterschied, als sich bei der niedersächsischen Genehmigungsbehörde die kürzeren (bis zu 6 Monaten) und besonders langen Verfahrensdauern (über 24 Monate) nahezu gleichmäßig auf Änderungs- und Neugenehmigungen verteilen, wohingegen im Süden vor allem die Änderungsgenehmigungen weniger mit kürzeren und häufiger mit besonders langen Verfahrensdauern einhergehen. Wie die vergleichende Analyse einzelner Verfahrensabschnitte (zu den einzelnen Phasen vgl. u.a. Tab. 46) auf der Basis von 
Häufigkeitsverteilungen sowie unter Heranziehung geläufiger statistischer Parameter (wie arithmetisches Mittel, Varianz, Median usw.) ergibt, kann dieser eben herausgestellte Unterschied nicht der Vorantragsphase (daran auch ablesbar, daß die $\mathrm{r}^{2}$-Werte in Tab. 46.1 für diese Phase in etwa gleich sind) zugerechnet, sondern muß vielmehr der vom Gesetzgeber zugestandenen »offiziellen Laufzeit« angelastet werden (vgl. Tab. 39 ff.). Nimmt man z.B. den Antragseingang als »offiziellen« Verfahrensbeginn, läßt sich dies leicht nachvollziehen. Wie den beiden Tabellen 46 und 46.1 zu entnehmen ist, nimmt bei beiden Modellen der 5. Verfahrensabschnitt der fachbehördlichen Abfassung der Stellungnahmen die meiste Zeit in Anspruch (mit einer geringfügigen Differenz zugunsten der süddeutschen Genehmigungsbehörde), wobei festzuhalten ist, daß die Chancen seitens der Genehmigungsbehörde, die insoweit autonomen Fachbehörden zur beschleunigten Bearbeitung anzuhalten, äußerst gering sind. Phase 2 der Vollständigkeitsprüfung sowie Phase 3 der Vorbereitung der Fachbehördenbeiziehung nehmen dagegen jeweils wenig Zeit in Anspruch. Allerdings erfährt die 2. Phase der Vollständigkeitsprüfung dadurch eine erhebliche zeitliche Entlastung, daß bei beiden Modellen in der dem eigentlichen Verfahren vorgeschalteten Vorantragsphase, die bei beiden Modellen jeweils beinahe so lange dauert wie das nachfolgende »offizielle« Verfahren (Tab. 46: Zeile 1, 7 u. 8), dic zur Vollständigkeitsprüfung erforderlichen Prüfschritte größtenteils bereits absolviert wurden. Auffällig sind dagegen die jeweiligen Unterschiede in Phase 4 der Fachbehördenbeiziehung und Phase 6 der Erarbeitung des Genehmigungsbescheids. In der Phase 6 »verbraucht « die niedersächsische Behörde 1,68 Monate (ca. 7 Wochen) und benötigt damit beinahe doppelt so viel Zeit als die süddeutsche Behörde mit 0,86 Monaten (ca. 3 1/2 Wochen). Umgekehrt ist in der 4. Phase der Fachbehördenbeiziehung die süddeutsche Behörde erheblich langsamer als die norddeutsche. Brachte diese diesen Verfahrensabschnitt in rund 7 Tagen hinter sich, so benötigte jene nicht ganz 2 1/2 Monate, bis auch die letzte der zu beteiligenden Fachbehörden zur Stellungnahme aufgefordert war. Für diesen Unterschied ließe sich der im niedersächsischen Modell mittelbar gegebene Vorteil verantwortlich machen, daß hier für eine Reihe zu beteiligender Fachämter eine zentrale Dienststelle als Adressat zur Verfügung steht, an die zeitaufwendige Koordinationsleistungen delegiert werden können. Die höhere Koordinationsbelastung im Süden vermag allerdings nicht den Befund zu erklären, daß dort die Genehmigungsbehörde in erster Linie bei Änderungsgenehmigungen vergleichsweise viel Zeit für die Behördenbeiziehung in Anspruch nahm (Tab. 42.12), indem sie in diesen Verfahren die Entscheidung über die zu beteiligenden Fachämter nicht zeitlich gebündelt, sondern nacheinander nach Maßgabe des weiteren Verfahrensverlaufs traf. Es ist nicht auszuschließen, daß sich dieses sukzessive Vorgehen auch einer gewissen Unsicherheit bei der genehmigungsrechtlichen Prüfung von Än- 
derungen bereits bestehender Anlagen verdankt. Eindeutige Anhaltspunkte für modellbedingte Ursachen dieser Divergenz lassen sich den Befunden nicht entnehmen. Von daher kommen für die Verzögerungen der Behördenbeiziehung bei der süddeutschen Genehmigungsbehörde auch »hausgemachte « (modellunabhängige) Defizite in der Selbstorganisation und in der Steuerung der behördeninternen Koordination und Kooperation in Frage. Wirken sich bei der norddeutschen Behörde die Koordinationserleichterungen der 4. Phase faktisch vorteilhaft fürs Zeitbudget aus, so wird dieses dagegen in der 6. Phase belastet durch die dort in der Hälfte der Fälle angewandte Praxis, dem Antragsteller/Betreiber einen Genehmigungsentwurf zuzusenden (Tab. 44; Tab. 44.1).

Mit Hilfe einer Korrelationsanalyse (Tab. 46.1), die die jeweilige Dauer der einzelnen Verfahrensschritte für beide Modelle mit der jeweiligen Gesamtdauer (ab Vorantragsberatung wie auch ab Antragseingang) in Beziehung setzt, läßt sich ein weiterer Unterschied zwischen beiden Genehmigungsmodellen aufzeigen, der nunmehr darauf zurückzuführen ist, daß jetzt für jede der beiden Behörden intern diejenige(n) Phase(n) ermittelt werden, die mit ihrem Zeitaufwand die Gesamtdauer besonders »belasten«. Wie der Tab. 46.1 entnommen werden kann, ist es bei der baden-württembergischen Genehmigungsbehörde erneut die 4. Phase der Fachbehördenbeiziehung, die hier zeitlich gesehen besonders ins Gewicht fällt, wohingegen nunmehr bei der niedersächsischen Genehmigungsbehörde die von ihr nur wenig beeinflußbare 5. Phase der fachbehördlichen Abfassung der Stellungnahmen den größten Zeitaufwand in Anspruch nimmt (wie bereits Tab. 46 erkennen läßt, ist im Durchschnitt der Zeitaufwand für diese Phase im Vergleich zu allen anderen Verfahrensschritten - ohne Vorantragsphase - am größten, wie auch der im Norden erzielte Durchschnittswert etwas über dem im Süden ermittelten liegt).

Im Ergebnis läßt sich aus der Gesamtverfahrensdauer weder für das eine noch für das andere Modell votieren, auch wenn in beiden Fällen jeweils ein gewisses Beschleunigungspotential vorhanden ist. Dies liegt bei der niedersächsischen Genehmigungsbehörde in der 6. Phase der Erarbeitung des Genehmigungsbescheids, bei ihrem süddeutschen Pendant in der 4. Phase der Fachbehördenbeiziehung.

$* * *$

Mit Blick auf die in den vereinfachten Verfahren erzielte »Vollzugsqualität« der beiden Genehmigungsmodelle läßt sich folgendes Fazit ziehen: Das baden-württembergische Modell begünstigt den Vollzug strengerer Umweltschutzanforderungen auf der ersten Vollzugsstufe, was einen höheren Kontrollbedarf auf der zweiten Stufe (die von der Aktenanalyse nicht 
erfaßt wurde) nach sich zieht, aber dort auch der Genehmigungsbehörde (Landratsamt) nachträglich Chancen zu Kompromissen einräumt. Die niedersächsische Lösung dagegen orientiert sich von vornherein pragmatisch an der zweiten Vollzugsstufe der ebenfalls vom Gew - beaufsichtsamt zu bewerkstelligenden Durchsetzung von Umweltschutzan orderungen, was sich auf der ersten Vollzugsstufe als Neigung zur größereı Kompromißbereitschaft bemerkbar macht; allerdings lassen sich solchermaßen zustandegekommene Lösungen mit Hilfe der in jedem zweiten Verfahren praktizierten »Endabnahme« prinzipiell korrigieren. Theoretisch betrachtet, scheinen im Süden infolge der dort vorgenommenen organisatorischen Trennung von Genehmigungs- und technischer Fachbehörde auch für die zweite Vollzugsstufe die günstigeren Rahmenbedingungen für die striktere Durchsetzung der auf der ersten Stufe erzielten »strengeren« Umweltschutzanforderungen vorzuliegen. Anders als im Norden ist im Süden die Genehmigungsbehörde auf der zweiten Stufe nicht dem unmittelbaren Druck seitens des Anlagenbetreibers ausgesetzt, was ihr eine vorteilhafte Stellung einräumt. Im »Schatten der Hierarchie (des Rechts)« kann sie sich, indem sie z.B. Fristen einräumt, einerseits kompromißbereit zeigen, andererseits kann sie jederzeit unter Berufung auf Recht und Gesetz auch »kompromißlos« entscheiden, wie überhaupt »der formell mögliche und im Konfliktfall nicht auszuschließende Oktroi (gerade auch dann, wenn er aus Sicht aller Beteiligten suboptimal wäre) eine außerordentlich wichtige Verhandlungsbedingung« darstellt, die »hartnäckiges Bargaining « letztlich nicht belohnt. ${ }^{413}$ Würde auch tatsächlich dieses theoretisch erwartete Verhalten mit dem beobachteten übereinstimmen, wäre dem baden-württembergischen Modell gegenüber dem niedersächsischen insgesamt eine größere Wirksamkeit (i.S. des Umweltschutzes) zuzusprechen.

Unabhängig vom Ausgang der zweiten Vollzugsstufe muß der Befund, der sich für die erste Stufe des baden-württembergischen Organisationsmodells ergibt, unter dem Gesichtspunkt der Koordination der beiden institutionellen Hauptakteure überraschen. Obwohl hier Genehmigungs- und technische Fachbehörde in einer auf Dauer angelegten engen Kooperationsbeziehung stehen, verhalten sie sich jeweils egoistisch und erzielen gerade auf diese Weise ein für den Umweltschutz günstiges Ergebnis. Wäre die Bedingung dauerhafter Kooperation nicht gegeben, erinnerte die Situation an das Gefangenendilemma. ${ }^{414}$

413 Scharpf 1991, S. 629. Vgl auch Bulling 1989, S. 289, mit dem Hinweis, daß kooperatives Verwaltungshandeln nur dann Staatsinteressen - wie hier dem Umweltschutz - zur Durchsetzung verhelfen kann, wenn »normierte einseitige Hoheitsmaßnahmen ,Flankenschutz« geben «.

$414 \mathrm{Vgl}$. Axelrod 1995, insbes. 7ff. 


\section{Literaturverzeichnis}

Arnold, P. (1989): Die Arbeit mit öffentlich-rechtlichen Verträgen im Umweltschutz beim Regierungspräsidium Stuttgart, in: Verwaltungsarchiv (VerwArch) 80, S. 125142.

Axelrod, R. (1995): Die Evolution der Kooperation. 3. Aufl., München/Wien.

Blankenburg, E. (1975): Die Aktenanalyse, in: Ders. (Hrsg.), Empirische Rechtssoziologie. München, S. 193-198.

Bohne, E. (1981): Der informale Rechtsstaat. Eine empirische und rechtliche Untersuchung zum Gesetzesvollzug unter besonderer Berücksichtigung des Immissionsschutzes. Berlin.

Bohne, E. (1994): Versicherungsmodelle zur Investitionsbeschleunigung und zum Abbau von Vollzugsdefiziten im Anlagenzulassungsrecht, in: Deutsches Verwaltungsblatt (DVB1.), S. 195-203.

Bulling, M. (1989): Kooperatives Verwaltungshandeln (Vorverhandlungen, Arrangements, Agreements und Verträge) in der Verwaltungspraxis, in: Die Öffentliche Verwaltung (DÖV) 42, S. 277-289.

Caspar, J. (1995): Ausverkauf oder Flexibilisierung des Umweltrechts?, in : Zeitschrift für Rechtspolitik (ZRP), S. 410-413.

Czajka, D. (1996): Kommentierung von $\S 7$ der 9. BImSchV, in: Feldhaus, G. (Hrsg.), Kommentar zur 9. BImSchV, 70. Erg.-Lfg., Dez.

Derlien, H.-U. (1982): Methodik der Verwaltungsforschung, in: Hesse, J. J. (Hrsg.), Politikwissenschaft und Verwaltungswissenschaft. Opladen, Sonderheft 13 der Politischen Vierteljahresschrift (PVS), S. 122-133.

Derlien, H.-U. (1987): Qualitatives und quantitatives Vorgehen in der Verwaltungsforschung, in: Koch, R. (Hrsg.), Verwaltungsforschung in Perspektive. Ein Colloquium zur Methode, zum Konzept und zum Transfer. Baden-Baden, S. 78-90.

Dose, N. (1994): Beschleunigung von Genehmigungsverfahren durch verbesserte Organisationsstrukturen der öffentlichen Verwaltung, in: Ders./Holznagel, B./Weber, V. (Hrsg.), Beschleunigung von Genehmigungsverfahren. Vorschläge zur Verbesserung des Industriestandortes Deutschland. Bonn, S. 219-241.

Dose, N. (1995): Kooperatives Handeln der Umweltschutzverwaltung, in: Ders./Voigt, R. (Hrsg.), Kooperatives Recht. Baden-Baden, S. 91-130.

Dose, N. (1997): Die verhandelnde Verwaltung. Eine empirische Untersuchung über den Vollzug des Immissionsschutzrechts. Baden-Baden.

Freudenberg, D. (1993): Die kranke Bezirksregierung (Teil 1), in: Verwaltungsführung, Organisation, Personalwesen (VOP), S. 234-237.

Friedberg, E. (1995): Ordnung und Macht. Dynamiken organisierten Handelns. Frankfurt a. Main, New York.

Hansmann, K. (Hrsg.) (1994): Bundesimmissionsschutzgesetz und ergänzende Vorschriftenrechtliche Genehmigungsverfahren. BImSchG vom 15. März 1974, zuletzt geändert durch das Gesetz vom 27.6.1994. Textausgabe mit Einführung und Anmerkungen. 14. Aufl., Baden-Baden. 
Harder, E. (1987): Das immissionsrechtliche Genehmigungsverfahren. Ein bürokratisches Investitionshemmnis? Melle.

Hellstern, G.-M. (1984): Verwaltungsvollzugsdaten und Aktenanalyse - ein tragfähiger Zugang zum Verständnis der Verwaltungswelt?, in: Werkzeuge qualitativer Stadtforschung, hrsg. im Auftr. der Robert Bosch Stiftung GmbH von H. Afheldt, W. Schultes, W. Siebel, T. Sieverts. Gerlingen, S. 199-224.

Hofmann, M. (1994): Beschleunigung von Genehmigungsverfahren: Aufgaben und Möglichkeiten einer obersten Landesbehörde, in: Dose, N./Holznagel, B./Weber, V. (Hrsg.), Beschleunigung von Genehmigungsverfahren. Vorschläge zur Verbesserung des Industriestandortes Deutschland. Bonn, S. 71-89.

Hucke, J./Wollmann, H. (1980): Methodenprobleme der Implementationsforschung, in: Mayntz, R. (Hrsg.), Implementation politischer Programme. Empirische Forschungsberichte. Königstein/Ts., S. 216-235.

Hucke, J./Wollmann, H. (1994): Vollzug des Umweltrechts, in: Handwörterbuch des Umweltrechts (HdUR), hrsg. von O. Kimminich, H. Frh. von Lersner, P.-Ch. Storm, 2. Aufl., Bd. II, Sp. 2694-2703.

Koch, H.-J. (1996): Beschleunigung, Deregulierung, Privatisierung. Modernisierung des Umweltrechts oder symbolische Standortpolitik?, in: Schlacke, S. (Hrsg.), Neue Konzepte im Umweltrecht: Beschleunigung durch Modernisierung, Effektivierung, Vereinfachung? Dokumentation der 3. Umweltfachtagung des Vereins für Umweltrecht e.V. am 9. und 10. Mai in Bremen. Berlin, S. 27-63.

Koch, H.-J. (1997): Beschleunigung, Deregulierung, Privatisierung: Modernisierung des Umweltrechts oder symbolische Standortpolitik? - Erster Teil -, in: Zeitschrift für angewandte Umweltforschung (ZAU) 10, S. 45-57.

Lorenz, A. (1991): Vollzugsdefizite im Umweltrecht, in: Umwelt- und Planungsrecht (UPR), S. 253-257.

Lübbe-Wolff, G. (1990): Organisation einer effektiven Umweltverwaltung, in: Institut für Umweltrecht / Arbeitskreis Umwelt und Recht (Hrsg.), Bundesdeutsches Umweltrecht - Perspektiven seiner Anwendung in der DDR. Tagungsbericht. Berlin, S. 1736.

Lübbe-Wolff, G. (1992): Das Kooperationsprinzip im Umweltrecht - Rechtsgrundsatz oder Deckmantel des Vollzugsdefizits?, in: Benz, A./Seibel, W. (Hrsg.), Zwischen Kooperation und Korruption. Abweichendes Verhalten in der Verwaltung. BadenBaden, S. 209-232.

Lübbe-Wolff, G. (1993): Vollzugsprobleme der Umweltverwaltung, in: Natur und Recht (NuR), S. 217-229.

Lübbe-Wolff, G. (1995): Beschleunigung von Genehmigungsverfahren auf Kosten des Umweltschutzes. Anmerkungen zum Bericht der Schlichter-Kommission, in: Zeitschrift für Umweltrecht (ZfU), S. 57-62.

Lübbe-Wolff, G. (1996): Modernisierung des Umweltordnungsrechts. Vollziehbarkeit Deregulierung - Effizienz. Bonn.

Mai, M. (1989): Die Bedeutung des fachspezifischen Habitus von Ingenieuren und Juristen in der wissenschaftlichen Politikberatung. Zur Soziologie von Technik und Recht unter besonderer Berücksichtigung des Bundes-Immissionsschutzgesetzes. Frankfurt a.M./Bern/ New York/Paris. 
Mayntz, R. (1980): Die Implementation politischer Programme. Theoretische Überlegungen zu einem neuen Forschungsgebiet, in: Dies. (Hrsg.), Implementation politischer Programme. Empirische Forschungsberichte. Königstein/Ts., S. 236-249.

Mayntz, R. (1997): Soziologie der öffentlichen Verwaltung. 4., durchges. Aufl., Heidelberg/Karlsruhe.

Mayntz, R./Derlien, H.-U./Bohne, E./Hesse, B./Hucke, J./Müller, A. (1978): Vollzugsprobleme der Umweltpolitik. Empirische Untersuchung der Implementation von Gesetzen im Bereich der Luftreinhaltung und des Gewässerschutzes. Stuttgart.

Pütz, M./Buchholz, K.-H. (1991): Die Genehmigungsverfahren nach dem BundesImmissionsschutzgesetz. Handbuch für Antragsteller und Genehmigungsbehörden mit Erläuterungen, Abwicklungshilfen und Beispielen. 4. Auf1., Berlin.

Scharpf, F. W. (1991): Die Handlungsfähigkeit des Staates am Ende des zwanzigsten Jahrhunderts, in: Politische Vierteljahresschrift (PVS) 32, S. 621-634.

Schlichter, O. (1995): Investitionsförderung durch flexible Genehmigungsverfahren, in: Deutsches Verwaltungsblatt (DVB1.), S. 173-179.

Schneider, H. (2000): Der Landkreis, in: Kommunalpolitik in Baden-Württemberg, hrsg. von Th. Pfizer u. H.-G. Wehling. 3., völlig überarb. u. erw. Aufl., Stuttgart, S. 238261.

Seibert, T.-M. (1981): Aktenanalysen. Zur Schriftform juristischer Deutungen. Tübingen.

Treiber, H. (1989): Regulative Politik in der Krise? Anmerkungen zu einem aktuellen Thema oder Reflexive Rationalität im Schatten des gesatzten Rechts, in: Ders., Vollzugskosten des Rechtsstaates und andere Studien zum Recht. Baden-Baden, S. 189217.

Winter, G. (1975): Das Vollzugsdefizit im Wasserrecht. Ein Beitrag zur Soziologie des öffentlichen Rechts. Berlin.

Wulfhorst, R. (1997): Erfahrungen mit den Beschleunigungsgesetzen - aus der Sicht der Exekutive, in: Verwaltungsarchiv (VerwArch) 88, S. 163-173. 


\section{Tabellenverzeichnis}

1.1: Anzahl der untersuchten vereinfachten Genehmigungsverfahren $\quad 19$

1.2: Jahr der Genehmigungserteilung der untersuchten Verfahren 20

1.3: Jahr der Antragstellung der untersuchten Verfahren 20

2.1: Anzahl der beteiligten Sachbearbeiter bei den Genehmigungsbe- 22 hörden

2.1-A: Zahl der Fälle je Sachbearbeiter bei der Genehmigungsbehörde $\quad 221 \mathrm{f}$. (Anhang)

2.2: Anzahl der beteiligten Sachbearbeiter bei den zuständigen

Gewerbeaufsichtsämtern

2.2-A: Zahl der Fälle je Sachbearbeiter beim Gewerbeaufsichtsamt (Anhang)

2.3: Beteiligung von Vorgesetzten bei der Verfahrensdurchfuhrung (Genehmigungsbehörden)

3: Anlagenart der Genehmigungsverfahren (nach den Ziff. des Anhangs der 4. BImSchV)

4: Unternehmensgröße der Antragsteller

5: $\quad$ Beratung des Antragstellers durch externen Sachverstand

5-A: Externe Beratung nach Unternehmensgröße (Kreuztabellierung) (Anhang)

6: $\quad$ Genehmigungsart der Verfahren

6-A: Genehmigungsart nach Unternehmensgröße (Kreuztabellierung) $227 \mathrm{f}$. (Anhang)

7: $\quad$ Auslöser der Verfahren

8: $\quad$ Anzahl an Vorantragsberatungen durch die Genehmigungsbehörde (Häufigkeitsverteilung)

9: $\quad$ Mitwirkung anderer Behörden bei der Vorantragsberatung

10: Medium der Kommunikation bei der behördlichen Vorantragsberatung

10.1: Ausgestaltung der behördlichen Vorantragsberatung in der Firma des Antragstellers (Anteile bezogen auf die Beratungsverfahren) 
10.2: Ausgestaltung der behördlichen Vorantragsberatung in der Firma des Antragstellers (Anteile bezogen auf alle Verfahren)

11: Behördliche Maßnahmen (Auswahl) als Gegenstand der Vorantragsberatung

12: Nachforderungen von Unterlagen im Rahmen der Vollständigkeitsprüfung (vor Fachbehördenbeteiligung)

13.1: Anzahl der in den Genehmigungsverfahren beteiligten Fachbehörden (Häufigkeitsverteilung)

13.2: Anzahl der in den Genehmigungsverfahren beteiligten Fachbehörden (komprimierte Häufigkeitsverteilung)

13.3: Anzahl der in den Genehmigungsverfahren beteiligten Fachbehörden (statistische Parameter)

14: Am häufigsten beteiligte Fachbehörden (Auswahl)

15: Anzahl der (dokumentierten) Kontakte zwischen Antragsteller und Genehmigungsbehörde nach Antragseingang (Häufigkeitsverteilung)

16.1: Anzahl der (dokumentierten) Kontakte zwischen Antragsteller und Genehmigungsbehörde ab Vorantragszeitraum bis Genehmigungsbescheid (Häufigkeitsverteilung)

16.2: Anzahl der (dokumentierten) Kontakte zwischen Antragsteller und Genehmigungsbehörde ab Vorantragszeitraum bis Genehmigungsbescheid (komprimierte Häufigkeitsverteilung)

16.3: Anzahl der Verfahren mit (kumulierten) Kontakten zwischen Antragsteller und Genehmigungsbehörde im Vorantragszeitraum und während der Antragsbearbeitung

17: Fachbehördenbeteiligung bei den Kontakten zwischen Genehmigungsbehörde und Antragsteller in der Zeit der Antragsbearbeitung

17.1: Direkte Fachbehördenbeteiligung bei den Kontakten zwischen Genehmigungsbehörde und Antragsteller ab Vorantragszeitraum bis Genehmigungsbescheid

18: Medium der Kommunikation zwischen Antragsteller und Genehmigungsbehörde nach Antragseingang

19: Gegenstände der Kommunikation (Auswahl) im Zeitraum der Antragsbearbeitung

20: Verfahren mit Betriebsbegehungen bzw. -kontrollen (Inspektionen) durch die Genehmigungsbehörde im Zeitraum der Antragsbearbeitung

21: Verfahren mit Betriebsbegehungen bzw. -kontrollen (Inspektionen) durch die Genehmigungsbehörde ab Vorantragszeitraum bis Genehmigungsbescheid 
21.1: Anzahl der Betriebsbegehungen bzw. -kontrollen (Inspektionen)

durch die Genehmigungsbehörde ab Vorantragszeitraum bis Genehmigungsbescheid

21a-A: Betriebskontrollen nach Unternehmensgröße (Kreuztabellierung) (Anhang)

21b-A: Betriebskontrollen nach sachlichen Kontroversen (Kreuztabellierung) (Anhang)

22: Häufigkeiten der Verfahren mit sachlichen Kontroversen zwischen den Hauptbeteiligten

22.1: Häufigkeiten der Verfahren mit sachlichen Kontroversen (gruppiert) zwischen den Hauptbeteiligten

23: Verfahrensbezogene Häufigkeiten immissionsschutzrechtlicher und nicht-immissionsschutzrechtlicher Einzelkontroversen zwischen den Hauptbeteiligten

23a-A: Sachliche Kontroversen nach Vorantragsberatung (Kreuztabellierung) (Anhang)

23b-A: Sachliche Kontroversen nach Unternehmensgröße (Kreuztabellierung) (Anhang)

23c-A: Sachliche Kontroversen nach Genehmigungsart (Kreuztabellierung) (Anhang)

23d-A: Sachliche Kontroversen nach Anlagenart (Kreuztabellierung) (Anhang)

23.1: Anzahl und statistische Parameter immissionsschutzrechtlicher und nicht-immissionsschutzrechtlicher Einzelkontroversen zwischen den Hauptbeteiligten

24: Gegenstände der Kontroversen in den Verfahren mit immissionsschutzrechtlichen (I-)Konflikten (Häufigkeiten)

24.1: Ausgang der immissionsschutzrechtlichen Einzelkontroversen

25: Gegenstände der Kontroversen in den Verfahren mit nichtimmissionsschutzrechtlichen (NI-)Konflikten (Häufigkeiten)

25.1: Ausgang der nicht-immissionsschutzrechtlichen Einzelkontroversen

26: Einschaltung vorgesetzter Behörden

26.1: Einschaltung vorgesetzter Behörden in immissionsschutzrechtlichen Konflikten

27: $\quad$ Entwurfsfassung des Genehmigungsbescheids nach Streitigkeit des Verfahrensverlaufs beim GAA-N

28: Abänderungswünsche des Antragstellers gegenüber der Entwurfsfassung des Genehmigungsbescheids beim GAA-N 
28.1: Behördliche Abhilfe« vom Antragsteller monierter Auflagen der Entwurfsfassung des Genehmigungsbescheids beim GAA-N

28.2: Behördliche $»$ Abhilfe« vom Antragsteller monierter Auflagen der Entwurfsfassung des Genehmigungsbescheids nach Auflagenart beim GAA-N

29: Widersprüche nach Entwurfsfassung des Genehmigungsbescheids beim GAA-N

30: Nicht-immissionsschutzrechtliche Auflagen (Auswahl - alphabetisch sortiert)

31: Immissionsschutzrechtliche Auflagen (ohne Meß- und Prüfnachweise)

32: Immissionsschutzrechtliche Nachweispflichten: Meß- und Prüfnachweise

33: Häufigkeit förmlicher Widersprüche gegen den Genehmigungsbescheid

34.1: Häufigkeit förmlicher Widersprüche gegen immissionsschutzrechtliche Auflagen des Genehmigungsbescheids

34.2: Häufigkeit förmlicher Widersprüche gegen sonstige, nichtimmissionsschutzrechtliche Auflagen des Genehmigungsbescheids

35.1: Ausgang förmlicher Widersprüche gegen immissionsschutzrechtliche Auflagen des Genehmigungsbescheids

35.2: Ausgang förmlicher Widersprüche gegen sonstige, nichtimmissionsschutzrechtliche Auflagen des Genehmigungsbescheids

36.1: Verfahrensdauer ab Vollständigkeit der Antragsunterlagen bis Erlaß Genehmigungsbescheid (Häufigkeitsverteilung)

36.11: Verfahrensdauer ab Vollständigkeit der Antragsunterlagen bis Erlaß Genehmigungsbescheid (statistische Parameter in Monaten)

36.2: Verfahrensdauer ab Vollständigkeit der Antragsunterlagen bis Erlaß Genehmigungsbescheid, getrennt nach Neu- und Änderungsgenehmigungen (Häufigkeitsverteilung)

36.21: Verfahrensdauer ab Vollständigkeit der Antragsunterlagen bis Erlaß Genehmigungsbescheid, getrennt nach Neu- und Änderungsgenehmigungen (statistische Parameter in Monaten)

37.1: Verfahrensdauer ab Antragseingang bis Erlaß Genehmigungsbescheid (Häufigkeitsverteilung)

37.11: Verfahrensdauer ab Antragseingang bis Erlaß Genehmigungsbescheid (statistische Parameter in Monaten) 
37.2: Verfahrensdauer ab Antragseingang bis Erlaß Genehmigungsbescheid, getrennt nach Neu- und Änderungsgenehmigungen (Häufigkeitsverteilung)

37.21: Verfahrensdauer ab Antragseingang bis Erlaß Genehmigungsbescheid, getrennt nach Neu- und Änderungsgenehmigungen (statistische Parameter in Monaten)

38.1: Verfahrensdauer ab erster Vorantragsberatung bis Erlaß 163 Genehmigungsbescheid (Häufigkeitsverteilung)

38.11: Verfahrensdauer ab erster Vorantragsberatung bis Erlaß Genehmigungsbescheid (statistische Parameter in Monaten)

38.2: Verfahrensdauer ab erster Vorantragsberatung bis Erlaß 165 Genehmigungsbescheid, getrennt nach Neu- und Änderungsgenehmigungen (Häufigkeitsverteilung)

38.21: Verfahrensdauer ab erster Vorantragsberatung bis Erlaß Genehmigungsbescheid, getrennt nach Neu- und Änderungsgenehmigungen (statistische Parameter in Monaten)

39: Dauer der Vorantragsberatung (Zeitspanne zwischen erster behördlicher Beratung und Antragseingang) (Häufigkeitsverteilung)

39.1: Dauer der Vorantragsberatung (Zeitspanne zwischen erster behördlicher Beratung und Antragseingang) (statistische Parameter in Monaten)

39.2: Dauer der Vorantragsberatung (Zeitspanne zwischen erster behördlicher Beratung und Antragseingang), getrennt nach Neuund Änderungsgenehmigungen (statistische Parameter in Monaten)

40: Dauer der Vollständigkeitsprüfung der Antragsunterlagen (Antragseingang bis Vollständigkeit der Unterlagen) (Häufigkeitsverteilung)

40.1: Dauer der Vollständigkeitsprüfung der Antragsunterlagen (Antragseingang bis Vollständigkeit der Unterlagen) (statistische Parameter in Monaten)

40.2-A: Dauer der Vollständigkeitsprüfung der Antragsunterlagen (Antragseingang bis Vollständigkeit der Unterlagen), getrennt nach Neu- und Änderungsgenehmigungen (statistische Parameter in Monaten) (Anhang)

41.1: Dauer der Vorbereitung der Fachbehördenbeiziehung (Vollständigkeit der Unterlagen bis Beiziehung der ersten Fachbehörde) (Häufigkeitsverteilung)

41.11: Dauer der Vorbereitung der Fachbehördenbeiziehung (Vollstän176 digkeit der Unterlagen bis Beiziehung der ersten Fachbehörde) (statistische Parameter in Monaten) 
41.12-A: Dauer der Vorbereitung der Fachbehördenbeiziehung (Vollständigkeit der Unterlagen bis Beiziehung der ersten Fachbehörde), getrennt nach Neu- und Änderungsgenehmigungen (statistische Parameter in Monaten) (Anhang)

41.2: Dauer der Fachbehördenbeiziehung (Beiziehung der ersten bis Beiziehung der letzten Fachbehörde) (Häufigkeitsverteilung)

41.21: Dauer der Fachbehördenbeiziehung (Beiziehung der ersten bis Beiziehung der letzten Fachbehörde) (statistische Parameter in Monaten)

41.22: Dauer der Fachbehördenbeiziehung (Beiziehung der ersten bis Beiziehung der letzten Fachbehörde), getrennt nach Neu- und Änderungsgenehmigungen (statistische Parameter in Monaten)

42.1: Gesamtdauer der Fachbehördenbeteiligung (erste angeforderte Stellungnahme bis Eingang der letzten Stellungnahme) (Häufigkeitsverteilung)

42.11: Gesamtdauer der Fachbehördenbeteiligung (erste angeforderte Stellungnahme bis Eingang der letzten Stellungnahme) (statistische Parameter in Monaten)

42.12: Gesamtdauer der Fachbehördenbeteiligung (erste angeforderte Stellungnahme bis Eingang der letzten Stellungnahme), getrennt nach Neu- und Änderungsgenehmigungen (statistische Parameter in Monaten)

42.2: (Mindest-) Dauer der fachbehördlichen Erarbeitung der Stellungnahmen (zuletzt beigezogene Fachbehörde bis Eingang der letzten fachbehördlichen Stellungnahme) (Häufigkeitsverteilung)

42.21: (Mindest-) Dauer der fachbehördlichen Erarbeitung der

Stellungnahmen (zuletzt beigezogene Fachbehörde bis Eingang der letzten fachbehördlichen Stellungnahme) (statistische Parameter in Monaten)

42.22: (Mindest-) Dauer der fachbehördlichen Erarbeitung der Stellungnahmen (zuletzt beigezogene Fachbehörde bis Eingang der letzten fachbehördlichen Stellungnahme), getrennt nach Neuund Änderungsgenehmigungen (statistische Parameter in Monaten)

43: Dauer der Erarbeitung des Genehmigungsbescheids (zuletzt eingegangene fachbehördliche Stellungnahme bis Ausfertigung des endgültigen Bescheids) (Häufigkeitsverteilung)

43.1: Dauer der Erarbeitung des Genehmigungsbescheids (zuletzt eingegangene fachbehördliche Stellungnahme bis Ausfertigung des endgültigen Bescheids) (statistische Parameter in Monaten) 
43.2: Dauer der Erarbeitung des Genehmigungsbescheids (zuletzt eingegangene fachbehördliche Stellungnahme bis Ausfertigung des endgültigen Bescheids), getrennt nach Neu- und Änderungsgenehmigungen (statistische Parameter in Monaten)

44: Dauer der Revisionsphase für die Entwurfsfassung des

Genehmigungsbescheids (Ausfertigung der Entwurfsfassung bis Ausfertigung des endgültigen Bescheids) - Nur GAA-N (Häufigkeitsverteilung und statistische Parameter in Monaten)

45: Dauer der Widerspruchsbearbeitung durch die Genehmigungsbehörde (Widerspruchseinlegung bis Widerspruchsentscheidung) (Häufigkeitsverteilung)

45.1: Dauer der Widerspruchsbearbeitung durch die Genehmigungsbehörde (Widerspruchseinlegung bis Widerspruchsentscheidung) (statistische Parameter in Monaten)

46: Übersicht über die durchschnittlichen Dauern der einzelnen Verfahrensabschnitte (arithmetische Mittel in Monaten)

46.1: Korrelationen der einzelnen Verfahrensabschnitte mit der Gesamtdauer - $\mathrm{r}^{2}$-Werte (erklärte Varianzen )

46.11-A: Korrelationen der einzelnen Verfahrensabschnitte mit der

Gesamtdauer - Korrelationskoeffizienten $\mathrm{r}$ (Anhang) 
VII. Anhang (ergänzende Tabellen)

Tab. 2.1-A

Zahl der Fälle je Sachbearbeiter bei der Genehmigungsbehörde

a) GAA-N

Value Label

\begin{tabular}{|c|c|r|r|c|}
\hline Value & Frequency & Percent & $\begin{array}{c}\text { Valid } \\
\text { Percent }\end{array}$ & $\begin{array}{c}\text { Cum } \\
\text { Percent }\end{array}$ \\
\hline 1 & 11 & 19,0 & 44,0 & 44,0 \\
\hline 2 & 2 & 3,4 & 8,0 & 52,0 \\
\hline 3 & 9 & 15,5 & 36,0 & 88,0 \\
\hline 4 & 1 & 1,7 & 4,0 & 92,0 \\
\hline 5 & 1 & 1,7 & 4,0 & 96,0 \\
\hline 7 & 1 & 1,7 & 4,0 & 100,0 \\
\hline 0 & 33 & 56,9 & Missing & \\
\hline \hline Total & 58 & 100,0 & 100,0 & \\
\hline
\end{tabular}

\begin{tabular}{|l|c||l|l||l|l|}
\hline Mean & 2,320 & Std err & 0,304 & Median & 2,000 \\
\hline Mode & 1,000 & Std dev & 1,520 & Variance & 2,310 \\
\hline Kurtosis & 2,349 & S E Kurt & 0,902 & Skewness & 1,341 \\
\hline S E Skew & 0,464 & Range & 6,000 & Minimum & 1,000 \\
\hline Maximum & 7,000 & & & & \\
\hline
\end{tabular}

\begin{tabular}{|c|c||c|c||c|c|}
\hline Percentile & Value & Percentile & Value & Percentile & Value \\
\hline 25,00 & 1,000 & 50,00 & 2,000 & 75,00 & 3,000 \\
\hline
\end{tabular}

\begin{tabular}{|l|l||l|l|}
\hline Valid cases & 25 & Missing cases & 33 \\
\hline
\end{tabular}


Tab. 2.1-A (Forts.)

b) LRA-I

Value Label

\begin{tabular}{|c|c|r|c|c|}
\hline Value & Frequency & Percent & $\begin{array}{c}\text { Valid } \\
\text { Percent }\end{array}$ & $\begin{array}{c}\text { Cum } \\
\text { Percent }\end{array}$ \\
\hline 2 & 1 & 2,3 & 20,0 & 20,0 \\
\hline 8 & 1 & 2,3 & 20,0 & 40,0 \\
\hline 9 & 2 & 4,7 & 40,0 & 80,0 \\
\hline 15 & 1 & 2,3 & 20,0 & 100,0 \\
\hline 0 & 38 & 88,4 & Missing & \\
\hline \hline Total & 43 & 100,0 & 100,0 & \\
\hline
\end{tabular}

\begin{tabular}{|l|r||l|r||l|c|}
\hline Mean & 8,600 & Std err & 2,064 & Median & 9,000 \\
\hline Mode & 9,000 & Std dev & 4,615 & Variance & 21,300 \\
\hline Kurtosis & 1,851 & S E Kurt & 2,000 & Skewness &,- 108 \\
\hline S E Skew & 0,913 & Range & 13,000 & Minimum & 2,000 \\
\hline Maximum & 15,000 & Sum & 43,000 & & \\
\hline
\end{tabular}

\begin{tabular}{|c|c||c|c||c|c|}
\hline Percentile & Value & Percentile & Value & Percentile & Value \\
\hline 25,00 & 5,000 & 50,00 & 9,000 & 75,00 & 12,000 \\
\hline
\end{tabular}

\begin{tabular}{|l|l||l|l|}
\hline Valid cases & 5 & Missing cases & 38 \\
\hline
\end{tabular}


Tab. 2.2-A

Zahl der Fälle je Sachbearbeiter beim Gewerbeaufsichtsamt

a) GAA-1 (zugehörig LRA-1)

Value Label

\begin{tabular}{|c|c|r|r|c|}
\hline Value & Frequency & Percent & $\begin{array}{c}\text { Valid } \\
\text { Percent }\end{array}$ & $\begin{array}{c}\text { Cum } \\
\text { Percent }\end{array}$ \\
\hline 1 & 9 & 20,9 & 47,4 & 47,4 \\
\hline 2 & 5 & 11,6 & 26,3 & 73,7 \\
\hline 3 & 1 & 2,3 & 5,3 & 78,9 \\
\hline 4 & 1 & 2,3 & 5,3 & 84,2 \\
\hline 5 & 2 & 4,7 & 10,5 & 94,7 \\
\hline 7 & 1 & 2,3 & 5,3 & 100,0 \\
\hline 0 & 24 & 55,8 & Missing & \\
\hline \hline Total & 43 & 100,0 & 100,0 & \\
\hline
\end{tabular}

\begin{tabular}{|l|c||l|r||l|r|}
\hline Mean & 2,263 & Std err & 0,404 & Median & 2,000 \\
\hline Mode & 1,000 & Std dev & 1,759 & Variance & 3,094 \\
\hline Kurtosis & 1,682 & S E Kurt & 1,014 & Skewness & 1,537 \\
\hline S E Skew & 0,524 & Range & 6,000 & Minimum & 1,000 \\
\hline Maximum & 7,000 & Sum & 43,000 & & \\
\hline
\end{tabular}

\begin{tabular}{|c|c||c|c||c|c|}
\hline Percentile & Value & Percentile & Value & Percentile & Value \\
\hline 25,00 & 1,000 & 50,00 & 2,000 & 75,00 & 3,000 \\
\hline
\end{tabular}

\begin{tabular}{|l|l||l|l|}
\hline Valid cases & 19 & Missing cases & 24 \\
\hline
\end{tabular}


Tab. 2.2-A (Forts.)

b) GAA-2 (zugehörig zu LRA-2)

Value Label

\begin{tabular}{|c|c|r|r|r|}
\hline Value & Frequency & Percent & $\begin{array}{c}\text { Valid } \\
\text { Percent }\end{array}$ & $\begin{array}{c}\text { Cum } \\
\text { Percent }\end{array}$ \\
\hline 1 & 8 & 33,3 & 57,1 & 57,1 \\
\hline 2 & 3 & 12,5 & 21,4 & 78,6 \\
\hline 3 & 2 & 8,3 & 14,3 & 92,9 \\
\hline 4 & 1 & 4,2 & 7,1 & 100,0 \\
\hline 0 & 10 & 41,7 & Missing & \\
\hline \hline Total & 24 & 100,0 & 100,0 & \\
\hline
\end{tabular}

\begin{tabular}{|l|c||l|c||l|l|}
\hline Mean & 1,714 & Std err & 0,266 & Median & 1,000 \\
\hline Mode & 1,000 & Std dev & 0,994 & Variance & 0,989 \\
\hline Kurtosis & 0,521 & S E Kurt & 1,154 & Skewness & 1,218 \\
\hline S E Skew & 0,597 & Range & 3,000 & Minimum & 1,000 \\
\hline Maximum & 4,000 & Sum & 24,000 & & \\
\hline
\end{tabular}

\begin{tabular}{|c|c||c|c||c|c|}
\hline Percentile & Value & Percentile & Value & Percentile & Value \\
\hline 25,00 & 1,000 & 50,00 & 1,000 & 75,00 & 2,250 \\
\hline
\end{tabular}

\begin{tabular}{|l|l||l|l|}
\hline Valid cases & 14 & Missing cases & 10 \\
\hline
\end{tabular}


Tab. 5-A

Externe Beratung (A7A1) nach Unternehmensgröße (A7B) - Kreuztabellierung

a) $G A A-N$

\begin{tabular}{|c|c|c|c|c|c|}
\hline \multirow[b]{2}{*}{ A7A1 (Beratung) } & \multirow[b]{2}{*}{$\begin{array}{c}\text { Count } \\
\text { Row Pct } \\
\text { Col Pct } \\
\text { Tot Pct }\end{array}$} & \multicolumn{3}{|c|}{$A 7 B$ (Unternehmensgröße) } & \multirow[b]{2}{*}{ Row Total } \\
\hline & & $\begin{array}{c}\text { Klein } \\
1\end{array}$ & $\begin{array}{c}\text { Mittel } \\
2\end{array}$ & $\begin{array}{c}\text { Gro } \beta \\
3\end{array}$ & \\
\hline keine & & $\begin{array}{r}1 \\
2,9 \\
25,0 \\
1,7\end{array}$ & $\begin{array}{r}11 \\
32,4 \\
44,0 \\
19,0\end{array}$ & $\begin{array}{r}22 \\
64,7 \\
75,9 \\
37,9\end{array}$ & $\begin{array}{r}34 \\
58,6\end{array}$ \\
\hline Nur Antrag & & $\begin{array}{r}2 \\
13,3 \\
50,0 \\
3,4\end{array}$ & $\begin{array}{r}9 \\
60,0 \\
36,0 \\
15,5\end{array}$ & $\begin{array}{r}4 \\
26,7 \\
13,8 \\
6,9\end{array}$ & $\begin{array}{r}15 \\
25,9\end{array}$ \\
\hline Nur Verfahren & & & & $\begin{array}{r}1 \\
100,0 \\
3,4 \\
1,7\end{array}$ & $\begin{array}{r}1 \\
1,7\end{array}$ \\
\hline Antrag und Verfahren & & $\begin{array}{r}1 \\
12,5 \\
25,0 \\
1,7\end{array}$ & $\begin{array}{r}5 \\
62,5 \\
20,0 \\
8,6 \\
\end{array}$ & $\begin{array}{r}2 \\
25,0 \\
6,9 \\
3,4\end{array}$ & $\begin{array}{r}8 \\
13,8\end{array}$ \\
\hline & $\begin{array}{c}\text { Column } \\
\text { Total }\end{array}$ & $\begin{array}{r}4 \\
6,9\end{array}$ & $\begin{array}{r}25 \\
43,1\end{array}$ & $\begin{array}{r}29 \\
50,0\end{array}$ & $\begin{array}{r}58 \\
100,0\end{array}$ \\
\hline
\end{tabular}

Number of Missing Observations: 0 
Tab. 5-A (Forts.)

b) LRA-1

\begin{tabular}{|c|c|c|c|c|c|}
\hline \multirow[b]{2}{*}{ A7A1 (Beratung) } & \multirow[b]{2}{*}{$\begin{array}{c}\text { Count } \\
\text { Row Pct } \\
\text { Col Pct } \\
\text { Tot Pct }\end{array}$} & \multicolumn{3}{|c|}{$A 7 B$ (Unternehmensgröße) } & \multirow[b]{2}{*}{ Row Total } \\
\hline & & $\begin{array}{c}\text { Klein } \\
I\end{array}$ & $\begin{array}{c}\text { Mittel } \\
2\end{array}$ & $\begin{array}{c}\mathrm{Gro} \beta \\
3\end{array}$ & \\
\hline \multicolumn{2}{|l|}{ keine } & $\begin{array}{r}5 \\
23,8 \\
35,7 \\
11,6\end{array}$ & $\begin{array}{r}8 \\
38,1 \\
47,1 \\
18,6\end{array}$ & $\begin{array}{r}8 \\
38,1 \\
66,7 \\
18,6\end{array}$ & $\begin{array}{r}21 \\
48,8\end{array}$ \\
\hline \multicolumn{2}{|l|}{ Nur Antrag } & $\begin{array}{r}5 \\
45,5 \\
35,7 \\
11,6\end{array}$ & $\begin{array}{r}5 \\
45,5 \\
29,4 \\
11,6\end{array}$ & $\begin{array}{r}1 \\
9,1 \\
8,3 \\
2,3\end{array}$ & $\begin{array}{r}11 \\
25,6\end{array}$ \\
\hline \multicolumn{2}{|l|}{ Nur Verfahren } & $\begin{array}{r}1 \\
100,0 \\
7,1 \\
2,3\end{array}$ & & & $\begin{array}{r}1 \\
2,3\end{array}$ \\
\hline \multicolumn{2}{|l|}{ Antrag und Verfahren } & $\begin{array}{r}3 \\
30,0 \\
21,4 \\
7,0\end{array}$ & $\begin{array}{r}4 \\
40,0 \\
23,5 \\
9,3\end{array}$ & $\begin{array}{r}3 \\
30,0 \\
25,0 \\
7,0\end{array}$ & $\begin{array}{r}10 \\
23,3\end{array}$ \\
\hline & $\begin{array}{c}\text { Column } \\
\text { Total }\end{array}$ & $\begin{array}{r}14 \\
32,6\end{array}$ & $\begin{array}{r}17 \\
39,5\end{array}$ & $\begin{array}{r}12 \\
27,9\end{array}$ & $\begin{array}{r}43 \\
100,0\end{array}$ \\
\hline
\end{tabular}

Number of Missing Observations: 0 
Tab. 6-A

Genehmigungsart (A4A1A) nach Unternehmensgröße (A7B)Kreuztabellierung

a) GAA-N

\begin{tabular}{|c|c|c|c|c|c|}
\hline A4AlA (Genehm. Art) & $\begin{array}{c}\text { Count } \\
\text { Row Pct } \\
\text { Col Pct } \\
\text { Tot Pct }\end{array}$ & $\begin{array}{c}\text { Klein } \\
1\end{array}$ & $\begin{array}{c}\text { Mittel } \\
2\end{array}$ & $\begin{array}{c}\text { Gro } \beta \\
3\end{array}$ & Row Total \\
\hline Neu-Genehmigung & & $\begin{array}{r}3 \\
18,8 \\
75,0 \\
5,2\end{array}$ & $\begin{array}{r}6 \\
37,5 \\
24,0 \\
10,3\end{array}$ & $\begin{array}{r}7 \\
43,8 \\
24,1 \\
12,1\end{array}$ & $\begin{array}{r}16 \\
27,6\end{array}$ \\
\hline $\begin{array}{l}\text { Änderungs- } \\
\text { Genehmigung }\end{array}$ & & $\begin{array}{r}1 \\
2,4 \\
25,0 \\
1,7\end{array}$ & $\begin{array}{r}19 \\
45,2 \\
76,0 \\
32,8\end{array}$ & $\begin{array}{r}22 \\
52,4 \\
75,9 \\
37,9\end{array}$ & $\begin{array}{r}42 \\
72,1\end{array}$ \\
\hline & $\begin{array}{c}\text { Column } \\
\text { Total }\end{array}$ & $\begin{array}{r}4 \\
6,9\end{array}$ & $\begin{array}{r}25 \\
43,1\end{array}$ & $\begin{array}{r}29 \\
50,0\end{array}$ & $\begin{array}{r}58 \\
100,0\end{array}$ \\
\hline
\end{tabular}

Number of Missing Observations: 0 
Tab. 6-A (Forts.)

b) LRA-1

\begin{tabular}{|c|c|c|c|c|c|}
\hline \multirow[b]{2}{*}{ A4AlA (Genehm. Art) } & \multirow[b]{2}{*}{$\begin{array}{c}\text { Count } \\
\text { Row Pct } \\
\text { Col Pct } \\
\text { Tot Pct }\end{array}$} & \multicolumn{3}{|c|}{$A 7 B$ (Unternehmensgröße) } & \multirow[b]{2}{*}{ Row Total } \\
\hline & & $\begin{array}{c}\text { Klein } \\
1\end{array}$ & $\begin{array}{c}\text { Mittel } \\
2\end{array}$ & $\begin{array}{c}\text { Gro } \beta \\
3\end{array}$ & \\
\hline Neu-Genehmigung & & $\begin{array}{r}9 \\
52,9 \\
64,3 \\
20,9\end{array}$ & $\begin{array}{r}3 \\
17,6 \\
17,6 \\
7,0\end{array}$ & $\begin{array}{r}5 \\
29,4 \\
41,7 \\
11,6\end{array}$ & $\begin{array}{r}17 \\
39,5\end{array}$ \\
\hline $\begin{array}{l}\text { Änderungs- } \\
\text { Genehmigung }\end{array}$ & & $\begin{array}{r}5 \\
19,2 \\
35,7 \\
11,6 \\
\end{array}$ & $\begin{array}{r}14 \\
53,8 \\
82,4 \\
32,6 \\
\end{array}$ & $\begin{array}{r}7 \\
26,9 \\
58,3 \\
16,3 \\
\end{array}$ & $\begin{array}{r}26 \\
60,5\end{array}$ \\
\hline & $\begin{array}{c}\text { Column } \\
\text { Total }\end{array}$ & $\begin{array}{r}14 \\
32,6\end{array}$ & $\begin{array}{r}17 \\
39,5\end{array}$ & $\begin{array}{r}12 \\
27,9\end{array}$ & $\begin{array}{r}43 \\
100,0\end{array}$ \\
\hline
\end{tabular}

Number of Missing Observations: 0 
Tab. 21a-A

Betriebskontrollen (C5BC) nach Unternehmensgröße (A7B)

a) $G A A-N$

\begin{tabular}{|c|c|c|c|c|c|}
\hline \multirow[b]{2}{*}{$\begin{array}{l}\text { C5BC } \\
\text { (Betriebskontrollen) }\end{array}$} & \multirow[b]{2}{*}{$\begin{array}{c}\text { Count } \\
\text { Row Pct } \\
\text { Col Pct } \\
\text { Tot Pct }\end{array}$} & \multicolumn{3}{|c|}{$A 7 B$ (Unternehmensgröße) } & \multirow[b]{2}{*}{ Row Total } \\
\hline & & $\begin{array}{c}\text { Klein } \\
1\end{array}$ & $\begin{array}{l}\text { Mittel } \\
2\end{array}$ & $\begin{array}{c}\text { Gro } \beta \\
3\end{array}$ & \\
\hline nein & & $\begin{array}{r}1 \\
3,2 \\
25,0 \\
1,7\end{array}$ & $\begin{array}{r}12 \\
38,7 \\
48,0 \\
20,7\end{array}$ & $\begin{array}{r}18 \\
58,1 \\
62,1 \\
31,0\end{array}$ & $\begin{array}{r}31 \\
53,4\end{array}$ \\
\hline ja & & $\begin{array}{r}3 \\
11,1 \\
75,0 \\
5,2\end{array}$ & $\begin{array}{r}13 \\
48,1 \\
52,0 \\
22,4\end{array}$ & $\begin{array}{r}11 \\
40,7 \\
37,9 \\
19,0\end{array}$ & $\begin{array}{r}27 \\
46,6\end{array}$ \\
\hline & $\begin{array}{l}\text { Column } \\
\text { Total }\end{array}$ & $\begin{array}{r}4 \\
6,9 \\
\end{array}$ & $\begin{array}{r}25 \\
43,1\end{array}$ & $\begin{array}{r}29 \\
50,0\end{array}$ & $\begin{array}{r}58 \\
100,0\end{array}$ \\
\hline
\end{tabular}

Number of Missing Observations: 0

b) LRA-1

\begin{tabular}{|c|c|c|c|c|c|}
\hline \multirow[b]{2}{*}{$\begin{array}{l}\text { C5BC } \\
\text { (Betriebskontrollen) }\end{array}$} & \multirow[b]{2}{*}{$\begin{array}{c}\text { Count } \\
\text { Row Pct } \\
\text { Col Pct } \\
\text { Tot Pct }\end{array}$} & \multicolumn{3}{|c|}{$A 7 B$ (Unternehmensgröße) } & \multirow[b]{2}{*}{ Row Total } \\
\hline & & $\begin{array}{c}\text { Klein } \\
1\end{array}$ & $\begin{array}{c}\text { Mittel } \\
2\end{array}$ & $\begin{array}{c}\text { Gro } \beta \\
3\end{array}$ & \\
\hline nein & & $\begin{array}{r}2 \\
10,0 \\
14,3 \\
4,7\end{array}$ & $\begin{array}{r}10 \\
50,0 \\
58,8 \\
23,3\end{array}$ & $\begin{array}{r}8 \\
40,0 \\
66,7 \\
18,6\end{array}$ & $\begin{array}{r}20 \\
46,5\end{array}$ \\
\hline ja & & $\begin{array}{r}12 \\
52,2 \\
85,7 \\
27,9\end{array}$ & $\begin{array}{r}7 \\
30,4 \\
41,2 \\
16,3\end{array}$ & $\begin{array}{r}4 \\
17,4 \\
33,3 \\
9,3\end{array}$ & $\begin{array}{r}23 \\
53,5\end{array}$ \\
\hline & $\begin{array}{l}\text { Column } \\
\text { Total }\end{array}$ & $\begin{array}{r}14 \\
32,6\end{array}$ & $\begin{array}{r}17 \\
39,5\end{array}$ & $\begin{array}{r}12 \\
27,9\end{array}$ & $\begin{array}{r}43 \\
100,0\end{array}$ \\
\hline
\end{tabular}

Number of Missing Observations: 0 
Tab. $21 b-A$

Betriebskontrollen (C5BC) nach sachlichen Kontroversen (C9AA)

a) $G A A-N$

\begin{tabular}{|c|c|c|c|c|c|c|}
\hline \multirow[b]{2}{*}{$\begin{array}{l}\text { C5BC } \\
\text { (Betriebskontrollen) }\end{array}$} & \multirow[b]{2}{*}{$\begin{array}{c}\text { Count } \\
\text { Row Pct } \\
\text { Col Pct } \\
\text { Tot Pct }\end{array}$} & \multicolumn{4}{|c|}{ C9AA (sachliche Kontroversen) } & \multirow[b]{2}{*}{$\begin{array}{l}\text { Row } \\
\text { Total }\end{array}$} \\
\hline & & $\begin{array}{c}\text { Keine } \\
0\end{array}$ & $\begin{array}{c}\text { Nur } \\
\text { NI-Streit } \\
1\end{array}$ & $\begin{array}{c}\text { Nur } \\
\text { I-Streit } \\
2\end{array}$ & $\begin{array}{c}\text { I- und NI- } \\
\text { Streit } \\
3\end{array}$ & \\
\hline \multirow{4}{*}{ nein } & & 12 & 6 & 9 & 4 & 31 \\
\hline & & 38,7 & 19,4 & 29,0 & 12,9 & 53,4 \\
\hline & & 54,5 & 42,9 & 90,0 & 33,3 & \\
\hline & & 20,7 & 10,3 & 15,5 & 6,9 & \\
\hline \multirow{4}{*}{ ja } & & 10 & 8 & 1 & 8 & 27 \\
\hline & & 37,0 & 29,6 & 3,7 & 29,6 & 46,6 \\
\hline & & 45,5 & 57,1 & 10,0 & 66,7 & \\
\hline & & 17,2 & 13,8 & 1,7 & 13,8 & \\
\hline & Column & 22 & 14 & 10 & 12 & 58 \\
\hline & Total & 37,9 & 24,1 & 17,2 & 20,7 & 100,0 \\
\hline
\end{tabular}

Number of Missing Observations: 0

b) $L R A-1$

\begin{tabular}{|c|c|c|c|c|c|c|}
\hline $\begin{array}{l}\text { C5BC } \\
\text { (Betriebskontrollen) }\end{array}$ & $\begin{array}{c}\text { Count } \\
\text { Row Pct } \\
\text { Col Pct } \\
\text { Tot Pct }\end{array}$ & $\begin{array}{c}\text { Keine } \\
0\end{array}$ & $\begin{array}{l}\text { A (sachlic } \\
\text { Nur } \\
\text { NI-Streit } \\
1\end{array}$ & $\begin{array}{l}\text { Kontrov } \\
\text { Nur } \\
\text { I-Streit } \\
2\end{array}$ & $\begin{array}{l}\text { sen) } \\
\begin{array}{l}\text { I- und NI- } \\
\text { Streit } \\
3\end{array}\end{array}$ & $\begin{array}{l}\text { Row } \\
\text { Total }\end{array}$ \\
\hline \multicolumn{2}{|l|}{ nein } & $\begin{array}{r}3 \\
15,0 \\
60,0 \\
7,0\end{array}$ & $\begin{array}{r}5 \\
25,0 \\
71,4 \\
11,6\end{array}$ & $\begin{array}{r}8 \\
40,0 \\
57,1 \\
18,6\end{array}$ & $\begin{array}{r}4 \\
20,0 \\
23,5 \\
9,3\end{array}$ & $\begin{array}{r}20 \\
46,5\end{array}$ \\
\hline \multicolumn{2}{|l|}{ ja } & $\begin{array}{r}2 \\
8,7 \\
40,0 \\
4,7\end{array}$ & $\begin{array}{r}2 \\
8,7 \\
28,6 \\
4,7\end{array}$ & $\begin{array}{r}6 \\
26,1 \\
42,9 \\
14,0\end{array}$ & $\begin{array}{r}13 \\
56,5 \\
76,5 \\
30,2\end{array}$ & $\begin{array}{r}23 \\
53,5\end{array}$ \\
\hline & $\begin{array}{c}\text { Column } \\
\text { Total }\end{array}$ & $\begin{array}{r}5 \\
11,6\end{array}$ & $\begin{array}{r}7 \\
16,3\end{array}$ & $\begin{array}{r}14 \\
32,6\end{array}$ & $\begin{array}{r}17 \\
39,5\end{array}$ & $\begin{array}{r}43 \\
100,0\end{array}$ \\
\hline
\end{tabular}

Number of Missing Observations: 0 
Tab. 23a-A

Sachliche Kontroversen (C9AA) nach Vorantragsberatung (B1A0)

a) $G A A-N$

\begin{tabular}{|c|c|c|c|c|c|}
\hline $\begin{array}{l}\text { C9AA (Sachliche } \\
\text { Kontroversen) }\end{array}$ & $\begin{array}{c}\text { Count } \\
\text { Row Pct } \\
\text { Col Pct } \\
\text { Tot Pct }\end{array}$ & $\begin{array}{c}\text { Keine } \\
0\end{array}$ & $\begin{array}{c}1-2 \\
1\end{array}$ & $\begin{array}{c}\text { uber } 2 \\
2\end{array}$ & Row Total \\
\hline Keine & & $\begin{array}{r}6 \\
27,3 \\
60,0 \\
10,3\end{array}$ & $\begin{array}{r}10 \\
45,5 \\
47,6 \\
17,2\end{array}$ & $\begin{array}{r}6 \\
27,3 \\
22,2 \\
10,3\end{array}$ & $\begin{array}{r}22 \\
37,9\end{array}$ \\
\hline Nur NI-Streit & & $\begin{array}{r}2 \\
14,3 \\
20,0 \\
3,4\end{array}$ & $\begin{array}{r}5 \\
35,7 \\
23,8 \\
8,6\end{array}$ & $\begin{array}{r}7 \\
50,0 \\
25,9 \\
12,1\end{array}$ & $\begin{array}{r}14 \\
24,1\end{array}$ \\
\hline Nur I-Streit & & $\begin{array}{r}2 \\
20,0 \\
20,0 \\
3,4\end{array}$ & $\begin{array}{r}2 \\
20,0 \\
9,5 \\
3,4\end{array}$ & $\begin{array}{r}6 \\
60,0 \\
22,2 \\
10,3\end{array}$ & $\begin{array}{r}10 \\
17,2\end{array}$ \\
\hline NI- und I-Streit & & & $\begin{array}{r}4 \\
33,3 \\
19,0 \\
6,9 \\
\end{array}$ & $\begin{array}{r}8 \\
66,7 \\
29,6 \\
13,8 \\
\end{array}$ & $\begin{array}{r}12 \\
20,7\end{array}$ \\
\hline & $\begin{array}{l}\text { Column } \\
\text { Total }\end{array}$ & $\begin{array}{r}10 \\
17,2\end{array}$ & $\begin{array}{r}21 \\
36,2\end{array}$ & $\begin{array}{r}27 \\
46,6\end{array}$ & $\begin{array}{r}58 \\
100,0\end{array}$ \\
\hline
\end{tabular}

Number of Missing Observations: 0 
Tab. 23a-A (Forts.)

b) LRA-1

\begin{tabular}{|c|c|c|c|c|c|}
\hline $\begin{array}{l}\text { C9AA (Sachliche } \\
\text { Kontroversen) }\end{array}$ & $\begin{array}{c}\text { Count } \\
\text { Row Pct } \\
\text { Col Pct } \\
\text { Tot Pct }\end{array}$ & $\begin{array}{c}B 1 A \\
\text { Keine } \\
0\end{array}$ & $\begin{array}{c}1-2 \\
1\end{array}$ & $\begin{array}{c}\text { über } 2 \\
2\end{array}$ & Row Total \\
\hline Keine & & & $\begin{array}{r}3 \\
60,0 \\
18,8 \\
7,0\end{array}$ & $\begin{array}{r}2 \\
40,0 \\
8,7 \\
4,7\end{array}$ & $\begin{array}{r}5 \\
11,6\end{array}$ \\
\hline Nur NI-Streit & & $\begin{array}{r}2 \\
28,6 \\
50,0 \\
4,7\end{array}$ & $\begin{array}{r}3 \\
42,9 \\
18,8 \\
7,0\end{array}$ & $\begin{array}{r}2 \\
28,6 \\
8,7 \\
4,7\end{array}$ & $\begin{array}{r}7 \\
16,3\end{array}$ \\
\hline Nur I-Streit & & $\begin{array}{r}1 \\
7,1 \\
25,0 \\
2,3\end{array}$ & $\begin{array}{r}4 \\
28,6 \\
25,0 \\
9,3\end{array}$ & $\begin{array}{r}9 \\
64,3 \\
39,1 \\
20,9\end{array}$ & $\begin{array}{r}14 \\
32,6\end{array}$ \\
\hline NI- und I-Streit & & $\begin{array}{r}1 \\
5,9 \\
25,0 \\
2,3\end{array}$ & $\begin{array}{r}6 \\
35,3 \\
37,5 \\
14,0\end{array}$ & $\begin{array}{r}10 \\
58,8 \\
43,5 \\
23,3\end{array}$ & $\begin{array}{r}17 \\
39,5\end{array}$ \\
\hline & $\begin{array}{c}\text { Column } \\
\text { Total }\end{array}$ & $\begin{array}{r}4 \\
9,3\end{array}$ & $\begin{array}{r}16 \\
37,2\end{array}$ & $\begin{array}{r}23 \\
53,5\end{array}$ & $\begin{array}{r}43 \\
100,0\end{array}$ \\
\hline
\end{tabular}

Number of Missing Observations: 0 
Tab. 23b-A

Sachliche Kontroversen (C9AA) nach Unternehmensgröße (A7B)

a) GAA-N

\begin{tabular}{|c|c|c|c|c|c|}
\hline $\begin{array}{l}\text { C9AA (Sachliche } \\
\text { Kontroversen) }\end{array}$ & $\begin{array}{c}\text { Count } \\
\text { Row Pct } \\
\text { Col Pct } \\
\text { Tot Pct }\end{array}$ & $\begin{array}{c}\text { Klein } \\
l\end{array}$ & $\begin{array}{c}\text { Mittel } \\
2\end{array}$ & $\begin{array}{c}\text { Gro } \beta \\
3\end{array}$ & Row Total \\
\hline Keine & & $\begin{array}{r}1 \\
4,5 \\
25,0 \\
1,7\end{array}$ & $\begin{array}{r}9 \\
40,9 \\
36,0 \\
15,5\end{array}$ & $\begin{array}{r}12 \\
54,5 \\
41,4 \\
20,7\end{array}$ & $\begin{array}{r}22 \\
37,9\end{array}$ \\
\hline Nur NI-Streit & & $\begin{array}{r}1 \\
7,1 \\
25,0 \\
1,7\end{array}$ & $\begin{array}{r}5 \\
35,7 \\
20,0 \\
8,6\end{array}$ & $\begin{array}{r}8 \\
57,1 \\
27,6 \\
13,8\end{array}$ & $\begin{array}{r}14 \\
24,1\end{array}$ \\
\hline Nur I-Streit & & . & $\begin{array}{r}5 \\
50,0 \\
20,0 \\
8,6\end{array}$ & $\begin{array}{r}5 \\
50,0 \\
17,2 \\
8,6\end{array}$ & $\begin{array}{r}10 \\
17,2\end{array}$ \\
\hline NI- und I-Streit & & $\begin{array}{r}2 \\
16,7 \\
50,0 \\
3,4\end{array}$ & $\begin{array}{r}6 \\
50,0 \\
24,0 \\
10,3\end{array}$ & $\begin{array}{r}4 \\
33,3 \\
13,8 \\
6,9\end{array}$ & $\begin{array}{r}12 \\
20,7\end{array}$ \\
\hline & $\begin{array}{l}\text { Column } \\
\text { Total }\end{array}$ & $\begin{array}{r}4 \\
6,9\end{array}$ & $\begin{array}{r}25 \\
43,1\end{array}$ & $\begin{array}{r}29 \\
50,0\end{array}$ & $\begin{array}{r}58 \\
100,0\end{array}$ \\
\hline
\end{tabular}

Number of Missing Observations: 0 
Tab. 23b-A (Forts.)

b) LRA-1

\begin{tabular}{|c|c|c|c|c|c|}
\hline $\begin{array}{l}\text { C9AA (Sachliche } \\
\text { Kontroversen) }\end{array}$ & $\begin{array}{c}\text { Count } \\
\text { Row Pct } \\
\text { Col Pct } \\
\text { Tot Pct }\end{array}$ & $\begin{array}{r}A 7 B \\
\text { Klein } \\
1\end{array}$ & $\begin{array}{c}\text { Mittel } \\
2\end{array}$ & $\begin{array}{c}\text { Gro } \beta \\
3\end{array}$ & Row Total \\
\hline Keine & & & $\begin{array}{r}4 \\
80,0 \\
23,5 \\
9,3\end{array}$ & $\begin{array}{r}1 \\
20,0 \\
8,3 \\
2,3\end{array}$ & $\begin{array}{r}5 \\
11,6\end{array}$ \\
\hline Nur NI-Streit & & $\begin{array}{r}3 \\
42,9 \\
21,4 \\
7,0\end{array}$ & $\begin{array}{r}2 \\
28,6 \\
11,8 \\
4,7\end{array}$ & $\begin{array}{r}2 \\
28,6 \\
16,7 \\
4,7\end{array}$ & $\begin{array}{r}7 \\
16,3\end{array}$ \\
\hline Nur I-Streit & & $\begin{array}{r}4 \\
28,6 \\
28,6 \\
9,3\end{array}$ & $\begin{array}{r}4 \\
28,6 \\
23,5 \\
9,3\end{array}$ & $\begin{array}{r}6 \\
42,9 \\
50,0 \\
14,0\end{array}$ & $\begin{array}{r}14 \\
32,6\end{array}$ \\
\hline NI- und I-Streit & & $\begin{array}{r}7 \\
41,2 \\
50,0 \\
16,3\end{array}$ & $\begin{array}{r}7 \\
41,2 \\
41,2 \\
16,3\end{array}$ & $\begin{array}{r}3 \\
17,6 \\
25,0 \\
7,0\end{array}$ & $\begin{array}{r}17 \\
39,5\end{array}$ \\
\hline & $\begin{array}{l}\text { Column } \\
\text { Total }\end{array}$ & $\begin{array}{r}14 \\
32,6\end{array}$ & $\begin{array}{r}17 \\
39,5\end{array}$ & $\begin{array}{r}12 \\
27,9\end{array}$ & $\begin{array}{r}43 \\
100,0\end{array}$ \\
\hline
\end{tabular}

Number of Missing Observations: 0 
Tab. $23 c-A$

Sachliche Kontroversen (C9AA) nach Genehmigungsart (A4A1A)

a) GAA-N

\begin{tabular}{|c|c|c|c|c|}
\hline \multirow[b]{2}{*}{$\begin{array}{l}\text { C9AA (Sachliche } \\
\text { Kontroversen) }\end{array}$} & \multirow[b]{2}{*}{$\begin{array}{c}\text { Count } \\
\text { Row Pct } \\
\text { Col Pct } \\
\text { Tot Pct }\end{array}$} & \multicolumn{2}{|c|}{ A4A1A (Genehmigungsart) } & \multirow[b]{2}{*}{ Row Total } \\
\hline & & $\begin{array}{c}\mathrm{Neu-} \\
\text { genehmigung } \\
1\end{array}$ & $\begin{array}{c}\text { Änderungs- } \\
\text { genehmigung } \\
2\end{array}$ & \\
\hline Keine & & $\begin{array}{r}6 \\
27,3 \\
37,5 \\
10,3\end{array}$ & $\begin{array}{r}16 \\
72,7 \\
38,1 \\
27,6\end{array}$ & $\begin{array}{r}22 \\
37,9\end{array}$ \\
\hline Nur NI-Streit & & $\begin{array}{r}5 \\
35,7 \\
31,3 \\
8,6\end{array}$ & $\begin{array}{r}9 \\
64,3 \\
21,4 \\
15,5\end{array}$ & $\begin{array}{r}14 \\
24,1\end{array}$ \\
\hline Nur I-Streit & & $\begin{array}{r}1 \\
10,0 \\
6,3 \\
1,7\end{array}$ & $\begin{array}{r}9 \\
90,0 \\
21,4 \\
15,5\end{array}$ & $\begin{array}{r}10 \\
17,2\end{array}$ \\
\hline NI- und I-Streit & & $\begin{array}{r}4 \\
33,3 \\
25,0 \\
6,9 \\
\end{array}$ & $\begin{array}{r}8 \\
66,7 \\
19,0 \\
13,8 \\
\end{array}$ & $\begin{array}{r}12 \\
20,7\end{array}$ \\
\hline & $\begin{array}{l}\text { Column } \\
\text { Total }\end{array}$ & $\begin{array}{r}16 \\
27,6\end{array}$ & $\begin{array}{r}42 \\
72,4\end{array}$ & $\begin{array}{r}58 \\
100,0\end{array}$ \\
\hline
\end{tabular}

Number of Missing Observations: 0 
Tab. 23c-A (Forts.)

b) LRA-1

\begin{tabular}{|c|c|c|c|c|}
\hline \multirow[b]{2}{*}{$\begin{array}{l}\text { C9AA (Sachliche } \\
\text { Kontroversen) }\end{array}$} & \multirow[b]{2}{*}{$\begin{array}{c}\text { Count } \\
\text { Row Pct } \\
\text { Col Pct } \\
\text { Tot Pct }\end{array}$} & \multicolumn{2}{|c|}{ A4A1A (Genehmigungsart) } & \multirow[b]{2}{*}{ Row Total } \\
\hline & & $\begin{array}{c}\text { Neu- } \\
\text { genehmigung } \\
I\end{array}$ & $\begin{array}{l}\text { Änderungs- } \\
\text { genehmigung } \\
\quad 2\end{array}$ & \\
\hline Keine & & . & $\begin{array}{r}5 \\
100,0 \\
19,2 \\
11,6\end{array}$ & $\begin{array}{r}5 \\
11,6\end{array}$ \\
\hline Nur NI-Streit & & $\begin{array}{r}4 \\
57,1 \\
23,5 \\
9,3\end{array}$ & $\begin{array}{r}3 \\
42,9 \\
11,5 \\
7,0\end{array}$ & $\begin{array}{r}7 \\
16,3\end{array}$ \\
\hline Nur I-Streit & & $\begin{array}{r}4 \\
28,6 \\
23,5 \\
9,3\end{array}$ & $\begin{array}{r}10 \\
71,4 \\
38,5 \\
23,3\end{array}$ & $\begin{array}{r}14 \\
32,6\end{array}$ \\
\hline NI- und I-Streit & & $\begin{array}{r}9 \\
52,9 \\
52,9 \\
20,9\end{array}$ & $\begin{array}{r}8 \\
47,1 \\
30,8 \\
18,6\end{array}$ & $\begin{array}{r}17 \\
39,5\end{array}$ \\
\hline & $\begin{array}{l}\text { Column } \\
\text { Total }\end{array}$ & $\begin{array}{r}17 \\
39,5\end{array}$ & $\begin{array}{r}26 \\
60,5\end{array}$ & $\begin{array}{r}43 \\
100,0\end{array}$ \\
\hline
\end{tabular}

Number of Missing Observations: 0 
Tab. $23 d-A$

Sachliche Kontroversen (C9AA) nach Anlagenart (A4B2A)

a) $G A A-N$

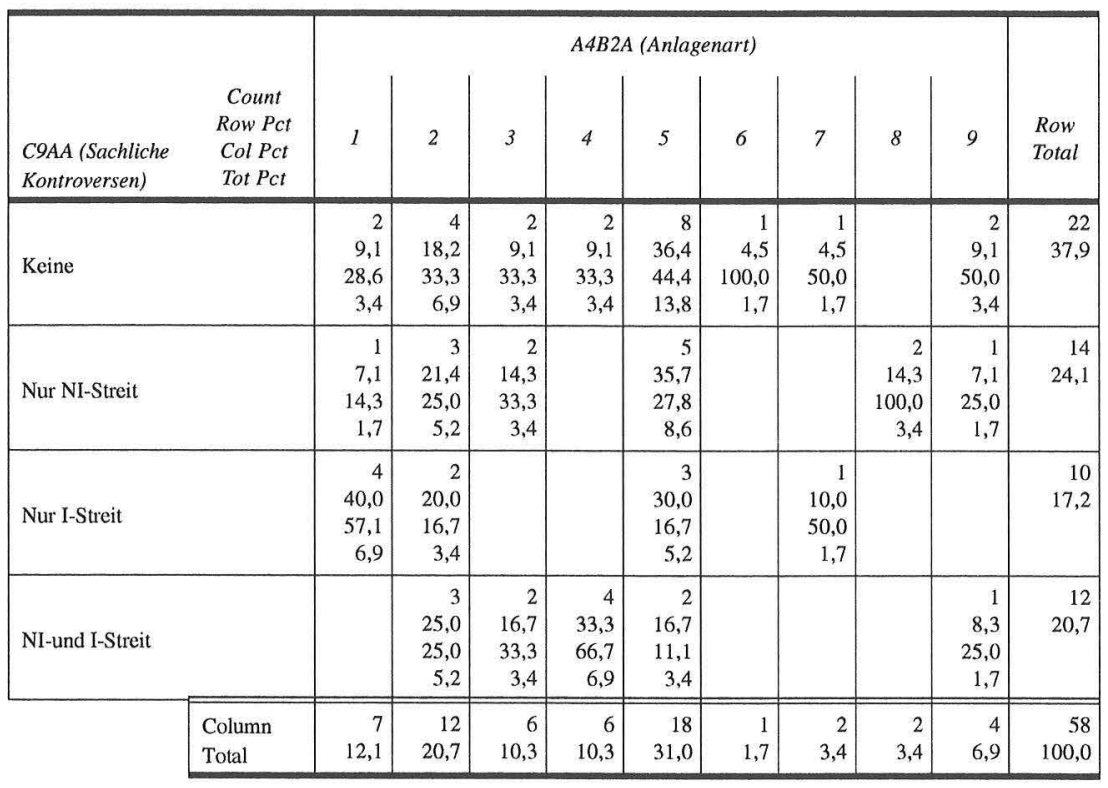

Number of Missing Observations: 0

Kategorie 10 der Anlagenart ist beim GAA-N nicht besetzt. Vgl. hierzu Tab. 3. 
Tab. 23d-A (Forts.)

b) $L R A-1$

\begin{tabular}{|c|c|c|c|c|c|c|c|c|c|c|c|}
\hline $\begin{array}{l}\text { C9AA (Sachliche } \\
\text { Kontroversen) }\end{array}$ & $\begin{array}{c}\text { Count } \\
\text { Row Pct } \\
\text { Col Pct } \\
\text { Tot Pct }\end{array}$ & 1 & 2 & 3 & $A 4 B 2$ & (Anlag & art) & 8 & 9 & 10 & $\begin{array}{l}\text { Row } \\
\text { Total }\end{array}$ \\
\hline Keine & & & $\begin{array}{r}1 \\
20,0 \\
14,3 \\
2,3\end{array}$ & $\begin{array}{r}1 \\
20,0 \\
50,0 \\
2,3\end{array}$ & $\begin{array}{r}1 \\
20,0 \\
11,1 \\
2,3\end{array}$ & & $\begin{array}{r}1 \\
20,0 \\
20,0 \\
2,3\end{array}$ & & $\begin{array}{r}1 \\
20,0 \\
16,7 \\
2,3\end{array}$ & & $\begin{array}{r}5 \\
11,6\end{array}$ \\
\hline Nur NI-Streit & & $\begin{array}{r}1 \\
14,3 \\
50,0 \\
2,3\end{array}$ & & & $\begin{array}{r}2 \\
28,6 \\
22,2 \\
4,7\end{array}$ & $\begin{array}{r}1 \\
14,3 \\
100,0 \\
2,3\end{array}$ & & $\begin{array}{r}1 \\
14,3 \\
25,0 \\
2,3\end{array}$ & & $\begin{array}{r}2 \\
28,6 \\
28,6 \\
4,7\end{array}$ & $\begin{array}{r}7 \\
16,3\end{array}$ \\
\hline Nur I-Streit & & $\begin{array}{r}1 \\
7,1 \\
50,0 \\
2,3\end{array}$ & $\begin{array}{r}1 \\
7,1 \\
14,3 \\
2,3\end{array}$ & $\begin{array}{r}1 \\
7,1 \\
50,0 \\
2,3\end{array}$ & $\begin{array}{r}2 \\
14,3 \\
22,2 \\
4,7\end{array}$ & & $\begin{array}{r}2 \\
14,3 \\
40,0 \\
4,7\end{array}$ & $\begin{array}{r}2 \\
14,3 \\
50,0 \\
4,7\end{array}$ & $\begin{array}{r}4 \\
28,6 \\
66,7 \\
9,3\end{array}$ & $\begin{array}{r}1 \\
7,1 \\
14,3 \\
2,3\end{array}$ & $\begin{array}{r}14 \\
32,6\end{array}$ \\
\hline NI-und I-Streit & & & $\begin{array}{r}5 \\
29,4 \\
71,4 \\
11,6\end{array}$ & & $\begin{array}{r}4 \\
23,5 \\
44,4 \\
9,3\end{array}$ & & $\begin{array}{r}2 \\
11,8 \\
40,0 \\
4,7\end{array}$ & $\begin{array}{r}1 \\
5,9 \\
25,0 \\
2,3\end{array}$ & $\begin{array}{r}1 \\
5,9 \\
10,7 \\
2,3\end{array}$ & $\begin{array}{r}4 \\
23,5 \\
57,1 \\
9,3\end{array}$ & $\begin{array}{r}17 \\
39,5 \\
-\end{array}$ \\
\hline & $\begin{array}{l}\text { Column } \\
\text { Total }\end{array}$ & $\begin{array}{r}2 \\
4,7\end{array}$ & $\begin{array}{r}7 \\
16,3\end{array}$ & $\begin{array}{r}2 \\
4,7\end{array}$ & $\begin{array}{r}9 \\
20,9\end{array}$ & $\begin{array}{r}1 \\
2,3\end{array}$ & $\begin{array}{r}5 \\
11,6\end{array}$ & $\begin{array}{r}4 \\
9,3\end{array}$ & $\begin{array}{r}6 \\
14,0\end{array}$ & $\begin{array}{r}7 \\
16,3\end{array}$ & $\begin{array}{r}43 \\
100,0\end{array}$ \\
\hline
\end{tabular}

Number of Missing Observations: 0

Kategorie 4 der Anlagenart ist beim LRA-1 nicht besetzt. Vgl. hierzu Tab. 3. 
Tab. 40.2-A

Dauer der Vollständigkeitsprüfung der Antragsunterlagen (Antragseingang bis Vollständigkeit der Unterlagen), getrennt nach Neu- und Änderungsgenehmigungen (statistische Parameter)*

\begin{tabular}{|l|c|c|c|c|c|c|}
\cline { 2 - 7 } & \multicolumn{2}{c|}{ AAA-N } & \multicolumn{2}{c|}{$L R A-1$} & \multicolumn{2}{c|}{ LRA-2 } \\
& And.-G & Neu-G & Änd.-G & Neu-G & Änd.-G & Neu-G \\
\hline Arith. Mittel & $\mathbf{0 , 3 9}$ & 0,44 & $\mathbf{0 , 3 4}$ & 0,14 & $\mathbf{0 , 2 2}$ & 1,21 \\
\hline Varianz & $\mathbf{0 , 4 1}$ & 0,63 & $\mathbf{1 , 0 4}$ & 0,13 & $\mathbf{0 , 2 8}$ & 2,55 \\
\hline Median & $\mathbf{0 , 0 0}$ & 0,00 & $\mathbf{0 , 0 0}$ & 0,00 & $\mathbf{0 , 0 0}$ & 0,52 \\
\hline Minimum & $\mathbf{0 , 0 0}$ & 0,00 & $\mathbf{0 , 0 0}$ & 0,00 & $\mathbf{0 , 0 0}$ & 0,00 \\
\hline Maximum & $\mathbf{2 , 5 2}$ & 2,72 & $\mathbf{4 , 1 0}$ & 1,34 & $\mathbf{1 , 5 1}$ & 4,52 \\
\hline \hline Gesamt & $\mathbf{4 1}$ & 15 & $\mathbf{2 4}$ & 17 & $\mathbf{1 2}$ & 10 \\
\hline
\end{tabular}

* Ohne Extremwerte.

Tab. 41.12-A

Dauer der Vorbereitung der Fachbehördenbeiziehung (Vollständigkeit der Unterlagen bis Beiziehung der ersten Fachbehörde) getrennt nach Neu- und Änderungsgenehmigungen (statistische Parameter in Monaten)*

\begin{tabular}{|c|c|c|c|c|c|c|}
\hline & \multicolumn{2}{|c|}{$G A A-N$} & \multicolumn{2}{|c|}{ LRA-1 } & \multicolumn{2}{|c|}{$L R A-2$} \\
\hline & Änd.-G & $\mathrm{Neu}-\mathrm{G}$ & Änd.-G & $\mathrm{Neu}-\mathrm{G}$ & Änd.-G & $\mathrm{Neu}-\mathrm{G}$ \\
\hline Arith. Mittel & 0,23 & 0,22 & 0,27 & 0,19 & 0,19 & 0,21 \\
\hline Varianz & 0,07 & 0,04 & 0,09 & 0,05 & 0,06 & 0,05 \\
\hline Median & 0,20 & 0,13 & 0,18 & 0,10 & 0,13 & 0,20 \\
\hline Minimum & 0,00 & 0,00 & $\mathbf{0 , 0 0}$ & 0,00 & 0,00 & 0,00 \\
\hline Maximum & 1,11 & 0,49 & 1,44 & 0,82 & 0,89 & 0,62 \\
\hline Gesamt & 31 & 11 & 20 & 16 & 11 & 6 \\
\hline
\end{tabular}

* Ohne Extremwerte und ohne die Verfahren, in denen bereits vor Feststellung der Vollständigkeit der Unterlagen die Behördenbeteiligung eingeleitet worden war (vgl. Zeile 1 in Tab. 41.1), beim GAA-N ferner abzüglich der zwei Fälle ohne Fachbehördenbeteiligung. 
Tab. 46.11-A

Korrelationen der einzelnen Verfahrensabschnitte mit der Gesamtdauer Korrelationskoeffizienten $\mathrm{r}$-*

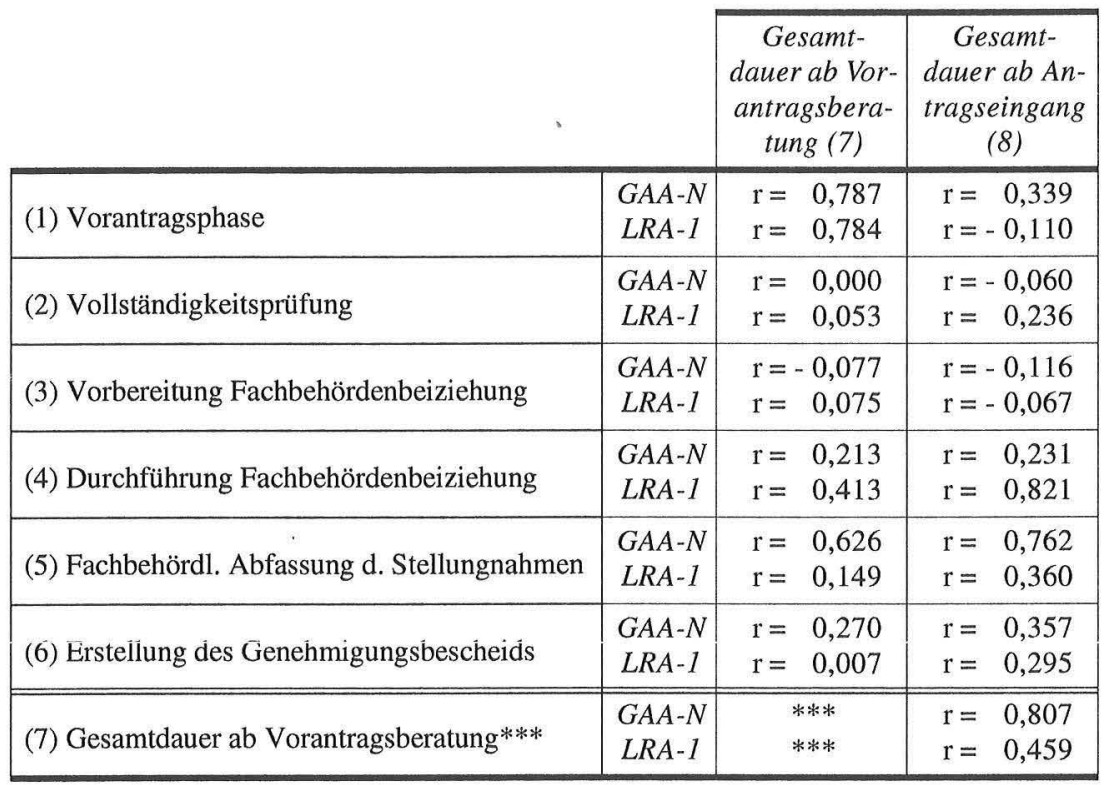

* Berechnung der Koeffizienten unter paarweisem Ausschluß von Extremwerten. Die Mindestzahl paarweise eingeschlossener Fälle beträgt 52 (GAA-N) bzw. 39 (LRA-1). 
